\title{
Anti-HIV Effects of Baculiferins Are Regulated by the Potential Target Protein DARS
}

Jianrong Liu ${ }^{\dagger}$, Ling Ma ${ }^{\ddagger}$, Chang Song ${ }^{\S}$, Hui Xing ${ }^{\S}$, Shan Cen ${ }^{\ddagger} *$, Wenhan Lin ${ }^{\dagger}, *$

${ }^{\dagger}$ State Key Laboratory of Natural and Biomimetic Drugs, Institute of Ocean Research, Ningbo Institute of Marine Medicine, Peking University, Beijing, P.R. China.

¥ Key Laboratory of Antiviral Drug Research, Institute of Medicinal Biotechnology, Chinese Academy of Medical Sciences and Peking Union Medical College, Beijing, P.R. China.

${ }^{\S}$ Division of Virology and Immunology National Center for AIDS/STD Control and Prevention (NCAIDS) Chinese Center for Disease Control and Prevention, Beijing, P.R. China

*Corresponding authors:

E-mail: whlin@bjmu.edu.cn (W. Lin), shancen@imb.pumc.edu.cn (S. Cen)

\section{Supporting Information}




\section{Experimental section}

General procedure: IR spectra (Infrared) were recorded on a Nicolet iS50 with attenuated total reflectance (ATR) accessory. NMR (nuclear magnetic resonance) spectra were recorded on a Bruker Avance-400FT NMR spectrometer. Chemical shifts $(\delta)$ were reported in parts per million (ppm). HRESIMS spectra (high-resolution mass spectrometry) were recorded on a Waters ${ }^{\circledR}$ Xevo G2 Q-Tof mass spectrometer. Chemical reagents and solvents were purchased from respective companies including Aladdin, Acros Organics, Alfa Aesar, J\&K, Macklin, TCI, Tong Guang and Sigma-Aldrich, and were used without further purification. Air and moisture sensitive reactions were carried out under high nitrogen atmosphere. The reaction was monitored by TLC (thin layer chromatography, silica GF254) supplied by Qingdao Marine Chemistry Co. Ltd. Silica gel (Silica G, 200-300 mesh) used for column chromatography was purchased from Qingdao Marine Chemistry Co. Ltd.
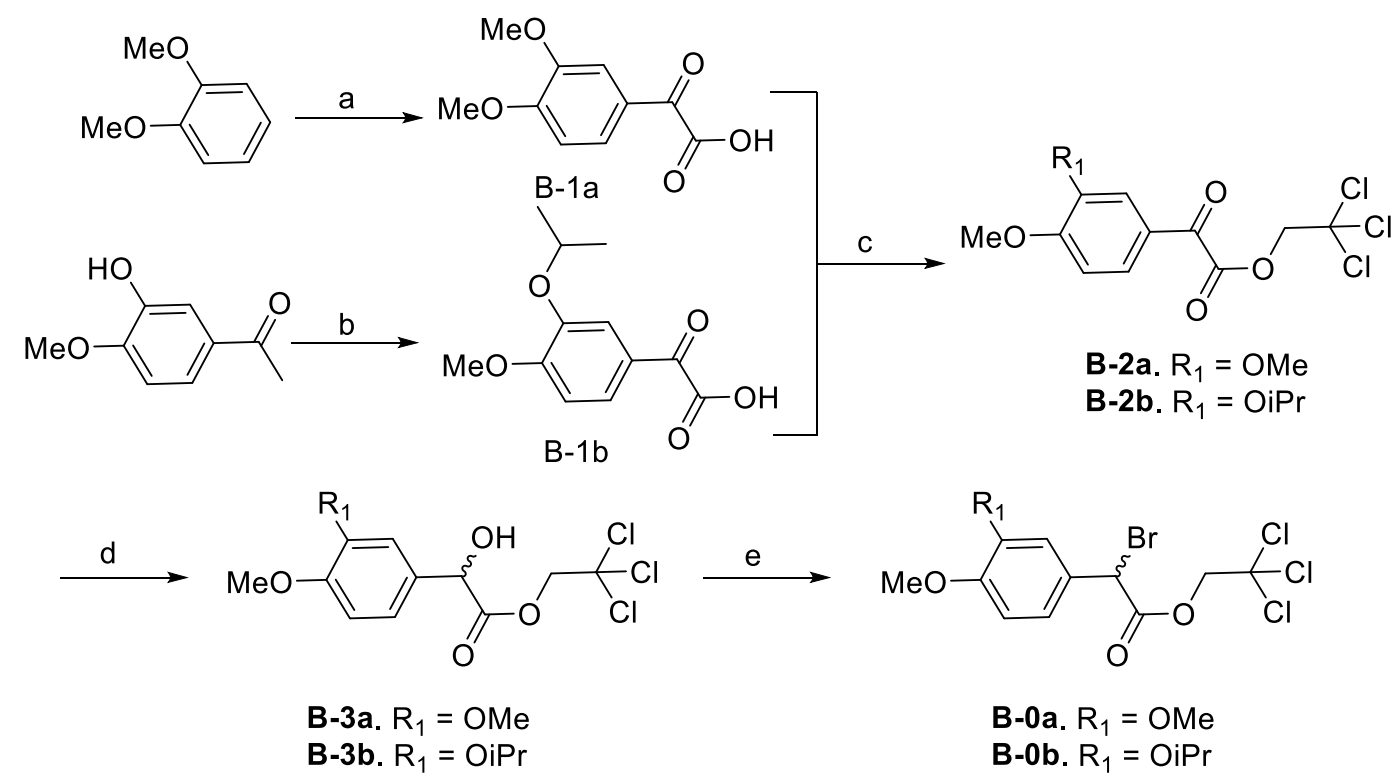
a) 1: methyl oxalyl chloride, $\mathrm{AlCl}_{3}, \mathrm{CH}_{2} \mathrm{Cl}_{2}, 0^{\circ} \mathrm{C}-\mathrm{rt}, 5 \mathrm{~h} ; 2 \mathrm{NaOH}, \mathrm{THF} / \mathrm{H}_{2} \mathrm{O}=2 / 3(\mathrm{v} / \mathrm{v})$, rt, $16 \mathrm{~h}$
b) 1: $\mathrm{K}_{2} \mathrm{CO}_{3}$, iPrBr, DMF, $60^{\circ} \mathrm{C}, 5 \mathrm{~h} ; 2: \mathrm{SeO}_{2}$, pyridine, reflux, $4 \mathrm{~h}$
c) $\mathrm{CCl}_{3} \mathrm{CH}_{2} \mathrm{OH}, \mathrm{DCC}, \mathrm{DMAP}, \mathrm{CH}_{2} \mathrm{Cl}_{2}$, rt, $3 \mathrm{~h}$
d) $\mathrm{NaBH} 4, \mathrm{THF} / \mathrm{H}_{2} \mathrm{O}=5 / 1(\mathrm{v} / \mathrm{v}),-5^{\circ} \mathrm{C}->0{ }^{\circ} \mathrm{C}, 1 \mathrm{~h}$
e) $\mathrm{POBr}_{3}, \mathrm{CH}_{2} \mathrm{Cl}_{2}, 0^{\circ} \mathrm{C}->\mathrm{rt}, 12 \mathrm{~h}$.

Scheme S1. Synthesis of intermediates B-0a and B-0b 


\section{Synthesis processes}
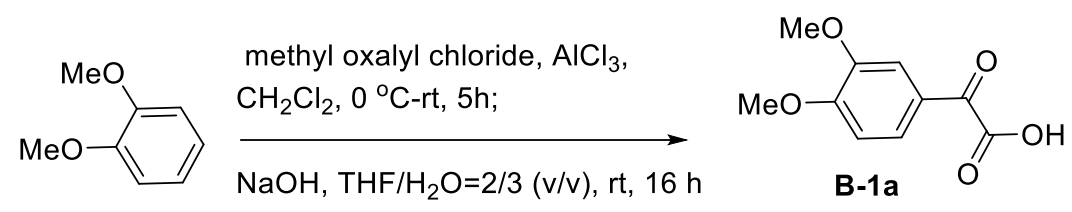

\section{Scheme S1-1. Synthesis of B-1a}

Synthesis of 2-(3,4-dimethoxyphenyl)-2-oxoacetic acid (B-1a): $\mathrm{AlCl}_{3}(1.16 \mathrm{~g}$, $8.7 \mathrm{mmol})$ was added to a mixture of methyloxalylchloride $(1.0 \mathrm{~g}, 8.2 \mathrm{mmol})$ and 1,2-dimethoxybenzene (1.0 g, $7.2 \mathrm{mmol}$ ) in dry $\mathrm{CH}_{2} \mathrm{Cl}_{2}$ at $0{ }^{\circ} \mathrm{C}$ under $\mathrm{N}_{2}$ to stir at $\mathrm{rt}$ for $5 \mathrm{~h}$. The reaction was quitted by ice $\mathrm{H}_{2} \mathrm{O}(50 \mathrm{~mL})$. The aqueous solution was extracted with $\mathrm{CH}_{2} \mathrm{Cl}_{2}(3 \times 50 \mathrm{~mL})$, while the organic layer was washed by brine (100 $\mathrm{mL})$ and dried over anhydrous $\mathrm{Na}_{2} \mathrm{SO}_{4}$. The extract containing methyl 2-(3,4-dimethoxyphenyl)-2-oxoacetate $(1.71 \mathrm{~g})$ was obtained under vacuo, and it was dissolved in tetrahydrofuran $(20 \mathrm{~mL})$ to add aqueous $\mathrm{NaOH}(15 \%, 30 \mathrm{~mL})$ stirring at rt overnight. After the $\mathrm{pH}$ value was adjusted to $3-4$ by $\mathrm{HCl}(1 \mathrm{~N})$, the mixture was extracted with EtOAc $(3 \times 50 \mathrm{~mL})$, and the organic layer was washed by brine $(100 \mathrm{~mL})$ and was dried over $\mathrm{Na}_{2} \mathrm{SO}_{4}$ to yield B-1a (1.23 g, 81\%).

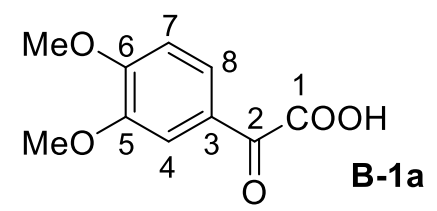

B-1a: IR $v_{\max } 3479,2940,1732,1667,1268 \mathrm{~cm}^{-1} ;{ }^{1} \mathrm{H}$ NMR $\left(\mathrm{CDCl}_{3}, 400 \mathrm{MHz}\right) \delta_{\mathrm{H}}$ 9.54 (bs, COOH), 8.17 (dd, $J=1.9,8.5 \mathrm{~Hz}, \mathrm{H}-8), 7.77$ (d, $J=1.9 \mathrm{~Hz}, \mathrm{H}-4), 6.94$ (d, $J$ $=8.5 \mathrm{~Hz}, \mathrm{H}-7), 3.98(\mathrm{~s}, \mathrm{OMe}), 3.94(\mathrm{~s}, \mathrm{OMe}) ;{ }^{13} \mathrm{C} \mathrm{NMR}\left(\mathrm{CDCl}_{3}, 100 \mathrm{MHz}\right) \delta_{\mathrm{C}} 182.4$ (C-2), 162.2 (C-1), 156.0 (C-5), 149.4 (C-6), 128.2 (C-3), 124.9 (C-8), 112.3 (C-4), 110.6 (C-7), 56.4 (OMe), 56.2 (OMe); HRESIMS m/z 209.0461 [M-H] (calcd. for $\mathrm{C}_{10} \mathrm{H}_{9} \mathrm{O}_{5}$ 209.0528).

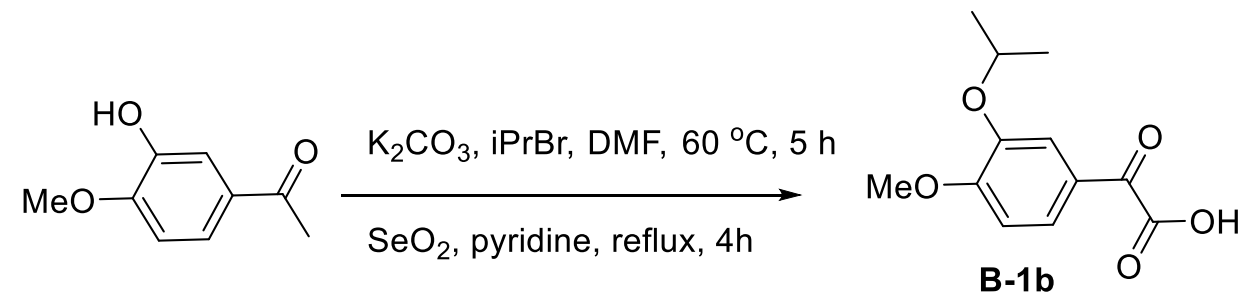

Scheme S1-2. Synthesis of B-1b 


\section{Synthesis of 2-(3-isopropoxy-4-methoxyphenyl)-2-oxoacetic acid (B-1b):}

2-Bromopropane $(856 \mu \mathrm{L}, 9.0 \mathrm{mmol})$ and $\mathrm{K}_{2} \mathrm{CO}_{3}(1.66 \mathrm{~g}, 12.0 \mathrm{mmol})$ were added to a solution of 1-(3-hydroxy-4-methoxyphenyl)ethanone $(1.0 \mathrm{~g}, 6.0 \mathrm{mmol})$ in dry DMF under $\mathrm{N}_{2}$. The mixture was stirred at $60{ }^{\circ} \mathrm{C}$ for $5 \mathrm{~h}$. After cooling to $\mathrm{rt}, \mathrm{H}_{2} \mathrm{O}(60 \mathrm{~mL})$ was added. The mixture was extracted with EtOAc $(3 \times 50 \mathrm{~mL})$, and the organic layer was washed by brine $(3 \times 100 \mathrm{~mL})$ and was dried over $\mathrm{Na}_{2} \mathrm{SO}_{4}$ to obtain 1-(3-isopropoxy-4-methoxyphenyl)ethanone (1.2 g, yield 96\%). Selenium dioxide (400 mg, $3.6 \mathrm{mmol}$ ) was added to 1-(3-isopropoxy-4-methoxyphenyl) ethanone (500 $\mathrm{mg}, 2.4 \mathrm{mmol})$ in pyridine $(10 \mathrm{~mL})$ to reflux for $4 \mathrm{~h}$ and then to stir at $\mathrm{rt}$ overnight. After concentration under vacuo, the residue was diluted with $\mathrm{H}_{2} \mathrm{O}(10 \mathrm{~mL})$ to adjust $\mathrm{pH}$ value to $1-2$ by $\mathrm{HCl}(1 \mathrm{~N})$. The solution was extracted by EtOAc $(3 \times 15 \mathrm{~mL})$, while the organic layer was washed with brine $(50 \mathrm{~mL})$ to obtain $\mathbf{B}-\mathbf{1 b}(552 \mathrm{mg}$, yield $96 \%$.

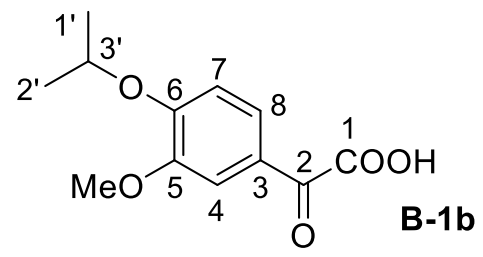

B-1b: IR $v_{\max } 3258,2978,1738,1668,1261 \mathrm{~cm}^{-1} ;{ }^{1} \mathrm{H} \mathrm{NMR}\left(\mathrm{CDCl}_{3}, 400 \mathrm{MHz}\right)$ $\delta_{\mathrm{H}} 9.16(\mathrm{bs}, \mathrm{COOH}), 8.03(\mathrm{dd}, J=2.0,8.5 \mathrm{~Hz}, \mathrm{H}-8), 7.76$ (d, $\left.J=2.0 \mathrm{~Hz}, \mathrm{H}-4\right), 6.93$ (d, $J=8.5 \mathrm{~Hz}, \mathrm{H}-7), 4.60$ (m, H-3'), 3.94 (s, OMe), 1.38 (d, $J=6.1 \mathrm{~Hz}, \mathrm{H}_{3}-1$ ' and $\mathrm{H}_{3}-2$ '); ${ }^{13} \mathrm{C}$ NMR $\left(\mathrm{CDCl}_{3}, 100 \mathrm{MHz}\right) \delta_{\mathrm{C}} 183.0$ (C-2), 163.1 (C-1), 156.9 (C-6), 147.6 (C-5), 127.6 (C-3), 124.9 (C-8), 115.6 (C-4), 111.0 (C-7), 71.7 (C-3’), 56.3 (OMe), 21.9 (C-1', C-2'); HRESIMS m/z 237.0766 [M-H]' (calcd. for $\mathrm{C}_{12} \mathrm{H}_{13} \mathrm{O}_{5}$ 237.0841).

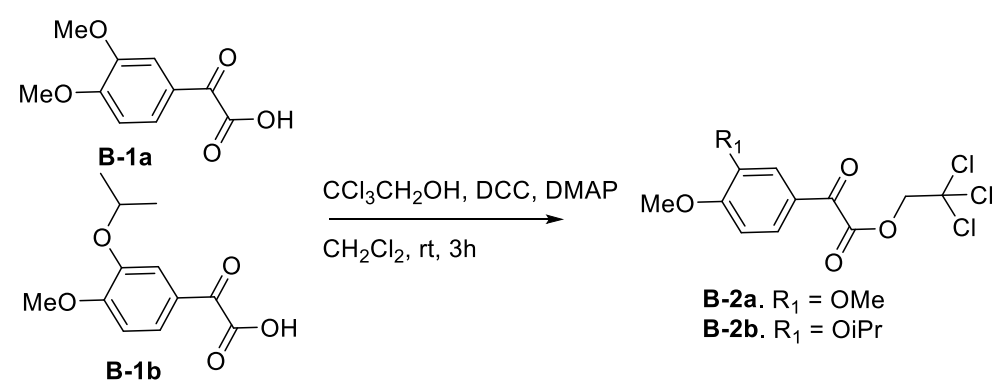

Scheme S1-3. Synthesis of B-2a and B-2b 


\section{Synthesis of 2,2,2-trichloroethyl-2-(3,4-dimethoxyphenyl)-2-oxoacetate (B-2a)}

and 2,2,2-trichloroethyl-2-(3-isopropoxy-4-methoxyphenyl)-2-oxoacetate (B-2b):

To a solution of B-1a (1.0 eq.) in dry $\mathrm{CH}_{2} \mathrm{Cl}_{2}$, dicyclohexylcarbodiimide (1.1 eq.), 4-dimethylaminopyridine (0.1 eq.) and 2,2,2-trichloroethanol (2.5 eq.) were added. The mixture was stirred at $\mathrm{rt}$ under $\mathrm{N}_{2}$ for $48 \mathrm{~h}$. The solvent was then removed under vacuo, and the residue was purified by silica gel column (200-300 mesh) eluting with $\mathrm{CH}_{2} \mathrm{Cl}_{2}$ to yield $\mathbf{B - 2 a}$ (yield $85 \%$ ). Following the same protocol as for B-1a, B-2b (yield 32\%) was synthesized.
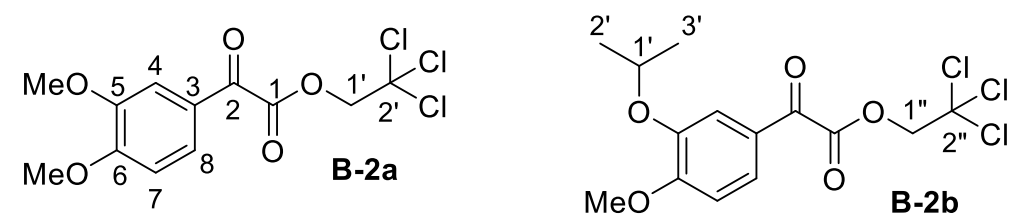

B-2a: Yellow solid, IR $v_{\max } 2960,1754,1671,1279 \mathrm{~cm}^{-1} ;{ }^{1} \mathrm{H} \mathrm{NMR}\left(\mathrm{CDCl}_{3}, 400\right.$ MHz) $\delta_{\mathrm{H}} 7.67(\mathrm{dd}, J=2.0,8.4 \mathrm{~Hz}, \mathrm{H}-8), 7.56(\mathrm{~d}, J=2.0 \mathrm{~Hz}, \mathrm{H}-4), 6.93$ (d, $J=8.4 \mathrm{~Hz}$, H-7), 5.01 (s, $\mathrm{H}_{2}-1$ '), 3.96 (s, OMe), 3.93 (s, OMe); ${ }^{13} \mathrm{C} \mathrm{NMR}\left(\mathrm{CDCl}_{3}, 100 \mathrm{MHz}\right) \delta_{\mathrm{C}}$ 183.3 (C-2), 162.3 (C-1), 155.5 (C-5), 149.7 (C-6), 126.5 (C-3), 125.2 (C-8), 110.8 (C-4), 110.5 (C-7), 94.2 (C-2'), 74.5 (C-1'), 56.4 (OMe), 56.2 (OMe); HRESIMS m/z $340.9743[\mathrm{M}+\mathrm{H}]^{+}$(calcd. for $\mathrm{C}_{12} \mathrm{H}_{12} \mathrm{Cl}_{3} \mathrm{O}_{5} 340.9672$ ).

B-2b: Yellow solid, IR $v_{\max } 2978,1745,1662,1228 \mathrm{~cm}^{-1} ;{ }^{1} \mathrm{H} \mathrm{NMR}\left(\mathrm{CDCl}_{3}, 400\right.$ MHz) $\delta_{\mathrm{H}} 7.64(\mathrm{dd}, J=1.9,8.5 \mathrm{~Hz}, \mathrm{H}-8), 7.56(\mathrm{~d}, J=1.9 \mathrm{~Hz}, \mathrm{H}-4), 6.93$ (d, $J=8.5 \mathrm{~Hz}$, H-7), 5.00 (s, H2-1'), 4.67 (m, H-1'), 3.93 (s, OMe), 1.37 (d, J=6.1 Hz, H3-2'/H3-3'); ${ }^{13} \mathrm{C}$ NMR $\left(\mathrm{CDCl}_{3}, 100 \mathrm{MHz}\right) \delta_{\mathrm{C}} 183.4$ (C-2), 162.4 (C-1), 156.7 (C-5), 147.9 (C-6), 126.2 (C-3), 125.1 (C-8), 114.4 (C-4), 111.0 (C-7), 94.2 (C-2”), 74.4 (C-1'), 71.6 (C-1”), 56.3 (OMe), 21.9 (C-2'/C-3'); HRESIMS m/z 369.1138 [M+H] ${ }^{+}$(calcd. for $\left.\mathrm{C}_{14} \mathrm{H}_{16} \mathrm{Cl}_{3} \mathrm{O}_{5} 368.9985\right)$.

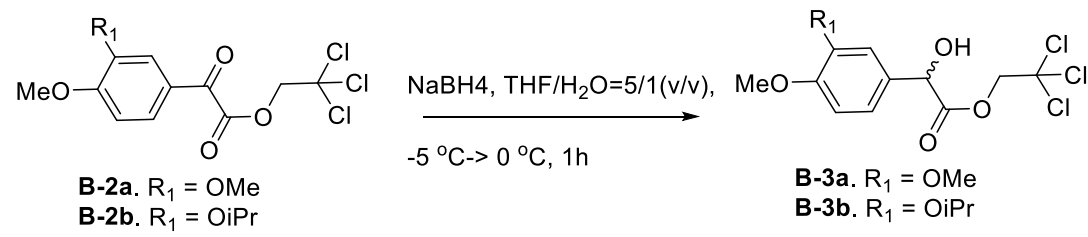




\section{Synthesis of 2,2,2-trichloroethyl-2-(3,4-dimethoxyphenyl)-2-hydroxyacetate}

(B-3a) and 2,2,2-trichloroethyl-2-hydroxy-2-(3-isopropoxy-4-methoxyphenyl) acetate (B-3b): Sodium borohydride (0.4 eq.) was slowly added to a solution of B-2a (1.0 eq.) in THF and $\mathrm{H}_{2} \mathrm{O}\left(\mathrm{THF} / \mathrm{H}_{2} \mathrm{O}=5 / 1, \mathrm{v} / \mathrm{v}\right)$ at $0{ }^{\circ} \mathrm{C}$. The temperature was increased slowly to rt during $30 \mathrm{~min}$. After stirring for additional $4 \mathrm{~h}$, the reaction was quitted by $\mathrm{H}_{2} \mathrm{O}(20 \mathrm{~mL})$ at $0{ }^{\circ} \mathrm{C}$. The mixture was extracted with EtOAc $(3 \times 20 \mathrm{~mL})$, while the organic layer was washed with brine $(60 \mathrm{~mL})$ and was purified by silica gel column eluting with $\mathrm{CH}_{2} \mathrm{Cl}_{2}$ to obtain B-3a (yield $80 \%$ ). B-3b (yield 55\%) was synthesized from $\mathbf{B - 2 b}$ by the same protocol as for $\mathbf{B - 3 a}$.
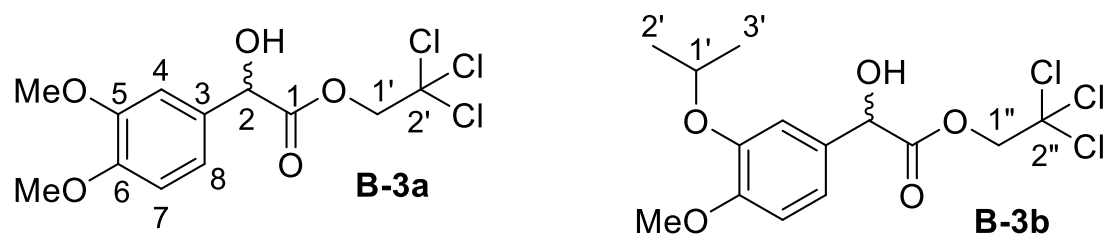

B-3a: Light yellow solid, IR $v_{\max } 3446,2971,1749,1705,1258 \mathrm{~cm}^{-1} ;{ }^{1} \mathrm{H}$ NMR $\left(\mathrm{CDCl}_{3}, 400 \mathrm{MHz}\right) \delta_{\mathrm{H}} 7.00(\mathrm{dd}, J=2.0,8.0 \mathrm{~Hz}, \mathrm{H}-8), 6.94(\mathrm{~d}, J=2.0 \mathrm{~Hz}, \mathrm{H}-4), 6.81$ (d, $J=8.0 \mathrm{~Hz}, \mathrm{H}-7), 5.25$ (s, H-2), 4.76 (d, $\left.J=11.9 \mathrm{~Hz}, \mathrm{Ha}-1^{\prime}\right), 4.71$ (d, $J=11.9 \mathrm{~Hz}$, $\left.\mathrm{Hb}-1^{\prime}\right), 3.83$ (s, OMe/OMe); ${ }^{13} \mathrm{C}$ NMR $\left(\mathrm{CDCl}_{3}, 100 \mathrm{MHz}\right) \delta_{\mathrm{C}} 172.1$ (C-1), 149.4 (C-5), 149.1 (C-6), 129.8 (C-3), 119.3 (C-8), 111.0 (C-4), 109.6 (C-7), 94.3 (C-2'), 74.6 (C-2), 72.7 (C-1'), 55.9 (OMe), 55.9 (OMe); ESIMS m/z 342.9907 [M+H]

$\boldsymbol{B}-3 \boldsymbol{b}$ : White solid, IR $v_{\max } 3447,2922,1750,1235 \mathrm{~cm}^{-1} ;{ }^{1} \mathrm{H}$ NMR $\left(\mathrm{CDCl}_{3}, 400\right.$ MHz) $\delta_{\mathrm{H}} 7.03(\mathrm{dd}, J=2.0,8.0 \mathrm{~Hz}, \mathrm{H}-8), 7.00(\mathrm{~d}, J=2.0 \mathrm{~Hz}, \mathrm{H}-4), 6.85(\mathrm{~d}, J=8.0 \mathrm{~Hz}$, H-7), 5.26 (s, H-2), 4.77 (s, H2-1”), 4.56 (m, H-1'), 3.84 (s, OMe), 1.35 (d, J=6.1 Hz, $\left.\mathrm{H}_{3}-2^{\prime} / \mathrm{H}_{3}-3^{\prime}\right) ;{ }^{13} \mathrm{C} \mathrm{NMR}\left(\mathrm{CDCl}_{3}, 100 \mathrm{MHz}\right) \delta_{\mathrm{C}} 172.3$ (C-1), 150.9 (C-5), 147.5 (C-6), 129.8 (C-3), 119.7 (C-8), 114.2 (C-4), 111.9 (C-7), 94.4 (C-2”), 74.7 (C-1'), 72.8 (C-2), 71.7 (C-1”), 56.1 (OMe), 22.2 (C-2'/C-3'); HRESIMS m/z 369.0189 [M-H]' (calcd. for $\mathrm{C}_{14} \mathrm{H}_{16} \mathrm{Cl}_{3} \mathrm{O}_{5} 369.0142$ ). 


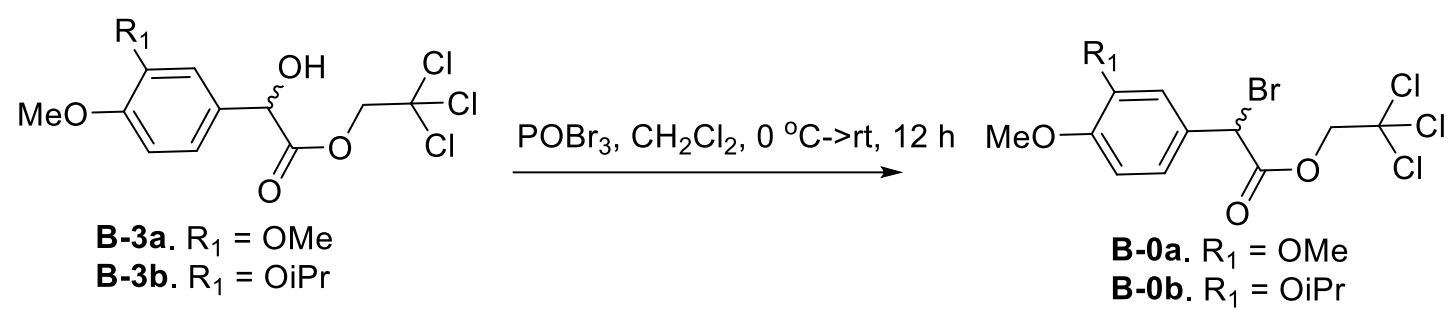

\section{Scheme S1-5. Synthesis of B-0a and B-0b}

\section{Synthesis of 2,2,2-trichloroethyl-2-bromo-2-(3,4-dimethoxyphenyl)acetate}

(B-0a) and 2,2,2-trichloroethyl-2-bromo-2-(3-isopropoxy-4-methoxyphenyl)

acetate (B-0b): Phosphorus oxybromide (2.0 eq.) was slowly added to a solution of B-3a (1.0 eq.) in a dry $\mathrm{CH}_{2} \mathrm{Cl}_{2}(10 \mathrm{~mL})$ under $\mathrm{N}_{2}$ at $\mathrm{rt}$. The mixture was stirred for $6 \mathrm{~h}$ in the dark. The reaction was quitted by $\mathrm{H}_{2} \mathrm{O}(50 \mathrm{~mL})$ at $0{ }^{\circ} \mathrm{C}$, while the mixture was extracted with $\mathrm{CH}_{2} \mathrm{Cl}_{2}(3 \times 30 \mathrm{~mL})$ and concentrated under vacuo. The residue was purified by silica gel column eluting with $\mathrm{CH}_{2} \mathrm{Cl}_{2}$ to yield B-0a (yield 78\%). B-0b was prepared from B-3b (1.0 eq.) by the same protocol as for B-0a with yield 85\%.
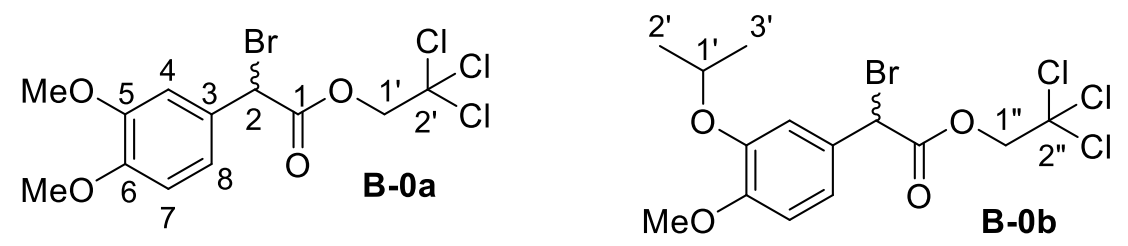

B-0a: Colorless oil, IR $v_{\max } 2957,1762,1264 \mathrm{~cm}^{-1} ;{ }^{1} \mathrm{H} \mathrm{NMR}\left(\mathrm{CDCl}_{3}, 400 \mathrm{MHz}\right)$ $\delta_{\mathrm{H}} 7.18(\mathrm{~d}, J=2.1 \mathrm{~Hz}, \mathrm{H}-4), 7.09$ (dd, $\left.J=8.3,2.1 \mathrm{~Hz}, \mathrm{H}-8\right), 6.80$ (d, $\left.J=8.3 \mathrm{~Hz}, \mathrm{H}-7\right)$, 5.44 (s, H-2), 4.82 (d, J=11.9 Hz, Ha-1'), 4.78 (d, J=11.9 Hz, Hb-1'), 3.90 (s, OMe), $3.87(\mathrm{~s}, \mathrm{OMe}) ;{ }^{13} \mathrm{C} \mathrm{NMR}\left(\mathrm{CDCl}_{3}, 100 \mathrm{MHz}\right) \delta_{\mathrm{C}} 167.0(\mathrm{C}-1), 150.4(\mathrm{C}-5), 149.4(\mathrm{C}-6)$, 127.0 (C-3), 121.7 (C-8), 111.9 (C-4), 110.9 (C-7), 94.4 (C-2'), 75.1 (C-1'), 56.1

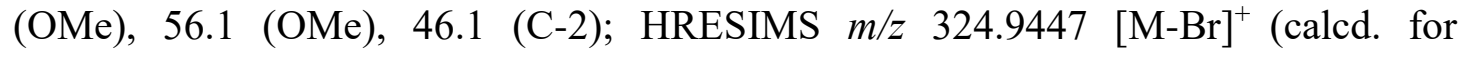
$\left.\mathrm{C}_{12} \mathrm{H}_{12} \mathrm{Cl}_{3} \mathrm{O}_{4} 324.9807\right)$.

$\boldsymbol{B}$-0b: Colorless oil, IR $v_{\max } 2975,1749,1236 \mathrm{~cm}^{-1} ;{ }^{1} \mathrm{H} \mathrm{NMR}\left(\mathrm{CDCl}_{3}, 400 \mathrm{MHz}\right)$ $\delta_{\mathrm{H}} 7.21(\mathrm{~d}, J=2.1 \mathrm{~Hz}, \mathrm{H}-4), 7.09$ (dd, $\left.J=8.3,2.1 \mathrm{~Hz}, \mathrm{H}-8\right), 6.82$ (d, $J=8.3 \mathrm{~Hz}, \mathrm{H}-7$ ), 5.42 (s, H-2), 4.80 (s, H2-1'), 4.60 (m, H-1'), 3.85 (s, OMe), 1.37 (d, J=6.1 Hz, $\left.\mathrm{H}_{3}-2^{\prime} / \mathrm{H}_{3}-3^{\prime}\right) ;{ }^{13} \mathrm{C} \mathrm{NMR}\left(\mathrm{CDCl}_{3}, 100 \mathrm{MHz}\right) \delta_{\mathrm{C}} 167.0$ (C-1), 151.5 (C-5), 147.5 (C-6), 126.9 (C-3), 121.9 (C-8), 116.1 (C-4), 111.6 (C-7), 94.4 (C-2”), 75.0 (C-1”), 71.8 (C-1'), 56.1 (OMe), 46.0 (C-2), 22.1 (C-2'), 22.0 (C-3'); HRESIMS m/z 353.0107 
$[\mathrm{M}-\mathrm{Br}]^{+}$(calcd. for $\mathrm{C}_{14} \mathrm{H}_{16} \mathrm{Cl}_{3} \mathrm{O}_{4} 353.0109$ ).<smiles>[R]c1ccc(C=O)cc1[R]</smiles>

$\mathrm{A}-1 \mathrm{~d} . \mathrm{R}_{3}=\mathrm{OMe}, \mathrm{R}_{4}=\mathrm{H}$

A-1e. $R_{3}=\mathrm{OMe}, \mathrm{R}_{4}=\mathrm{Cl}$

A-1f. $R_{3}=\mathrm{OMe}, \mathrm{R}_{4}=\mathrm{Br}$

A-1g. $R_{3}=O M e, R_{4}=O i P r$<smiles>[R]c1ccc(C=COC)cc1[R]</smiles>

A-2d. $\mathrm{R}_{3}=\mathrm{OMe}, \mathrm{R}_{4}=\mathrm{H}$

A-2e. $\mathrm{R}_{3}=\mathrm{OMe}, \mathrm{R}_{4}=\mathrm{Cl}$

A-2f. $R_{3}=\mathrm{OMe}, \mathrm{R}_{4}=\mathrm{Br}$

A-2g. $\mathrm{R}_{3}=\mathrm{OMe}, \mathrm{R}_{4}=\mathrm{OiPr}$

\section{Scheme S2. Synthesis of A-2d to A-2g.}

Synthesis of A-2d to A-2g: A-1d is a commercial product. A-1e and A-1f were derived from 4-methoxybenzaldehyde following the methods in literatures [1-2]. A-1g was derived from 3-hydroxy-4-methoxybenzaldehyde by the reported method [3]. (Methoxymethyl) triphenylphodphonium chloride (1.1 eq.) was added to a mixture of potassium $t$-butoxide (1.1 eq.) and A-1d (1.0 eq.) in dry tetrahydrofuran under $\mathrm{N}_{2}$ at 0 ${ }^{\circ} \mathrm{C}$ to stir at $\mathrm{rt}$ overnight. The reaction was quitted by $\mathrm{H}_{2} \mathrm{O}$, and the mixture was extracted with EtOAc. After concentration under vacuo, the EtOAc residue was purified by silica gel column eluting with hexane-acetone (1:1) to yield A-2d. A-2e to A-2g were derived from A-1e to A-1g respectively by the same protocol as for A-2d.

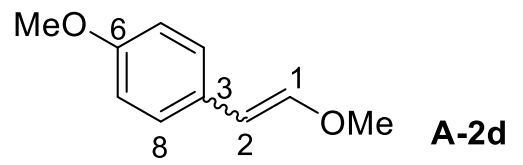

$\boldsymbol{A}-2 \boldsymbol{d}$ (yield 80\%): ${ }^{1} \mathrm{H}$ NMR $\left(\mathrm{CDCl}_{3}, 400 \mathrm{MHz}\right) \delta_{\mathrm{H}} 6.50(\mathrm{~d}, J=16.0 \mathrm{~Hz}, \mathrm{H}-1)$, $5.40(\mathrm{~d}, J=16.0 \mathrm{~Hz}, \mathrm{H}-2), 7.20(\mathrm{~d}, J=8.0 \mathrm{~Hz}, \mathrm{H}-4 / \mathrm{H}-8), 6.90(\mathrm{~d}, J=8.0 \mathrm{~Hz}$, H-5/H-7), 3.80 (s, OMe), 3.75 (OMe); ESIMS m/z $165.08[\mathrm{M}+\mathrm{H}]^{+}$.

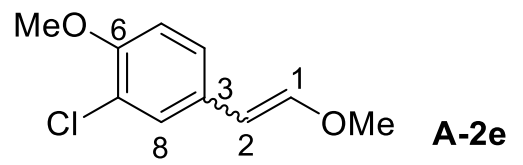

$\boldsymbol{A}-2 \boldsymbol{e}$ (yield 70\%): $E$-form: ${ }^{1} \mathrm{H}$ NMR $\left(\mathrm{CDCl}_{3}, 400 \mathrm{MHz}\right) \delta_{\mathrm{H}} 7.27(\mathrm{~d}, J=1.9 \mathrm{~Hz}$, H-8), 7.08 (dd, $J=8.5,1.9 \mathrm{~Hz}, \mathrm{H}-4), 6.95$ (d, $J=15.0 \mathrm{~Hz}, \mathrm{H}-1), 6.85$ (d, $J=8.5 \mathrm{~Hz}$, $\mathrm{H}-5), 5.73$ (d, $J=15.0 \mathrm{~Hz}, \mathrm{H}-2), 3.90$ (s, OMe), 3.69 (s, OMe); ${ }^{13} \mathrm{C} \mathrm{NMR}\left(\mathrm{CDCl}_{3}\right.$, $100 \mathrm{MHz}) \delta_{\mathrm{H}} 153.2$ (C-6), 148.6 (C-1), 130.2 (C-3), 126.8 (C-4), 124.6 (C-8), 122.7 (C-7), 112.5 (C-5), 103.7 (C-2), 56.7 (OMe), 56.4 (OMe). Z-form: ${ }^{1} \mathrm{H}$ NMR $\left(\mathrm{CDCl}_{3}\right.$, $400 \mathrm{MHz}) \delta_{\mathrm{H}} 7.68(\mathrm{~d}, J=2.0 \mathrm{~Hz}, \mathrm{H}-8), 7.37(\mathrm{dd}, J=8.5,2.0 \mathrm{~Hz}, \mathrm{H}-4), 6.83$ (d, $J=$ 
$8.5 \mathrm{~Hz}, \mathrm{H}-5), 6.07$ (d, $J=10.0 \mathrm{~Hz}, \mathrm{H}-1), 5.11$ (d, $J=10.0 \mathrm{~Hz}, \mathrm{H}-2), 3.86$ (s, OMe), $3.76(\mathrm{~s}, \mathrm{OMe}) ;{ }^{13} \mathrm{C} \mathrm{NMR}\left(\mathrm{CDCl}_{3}, 100 \mathrm{MHz}\right) \delta_{\mathrm{C}} 152.9$ (C-6), $147.4(\mathrm{C}-1), 129.8$ (C-3), 127.6 (C-4), 124.5 (C-8), 122.0 (C-7), 111.8 (C-5), 104.1 (C-2), 60.7 (OMe), 56.1 (OMe). ESIMS m/z $199.04[\mathrm{M}+\mathrm{H}]^{+}$.

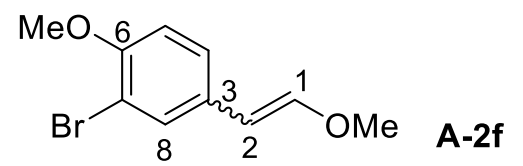

$\boldsymbol{A}-2 \boldsymbol{f}$ (yield 80\%): ${ }^{1} \mathrm{H}$ NMR $\left(\mathrm{CDCl}_{3}, 400 \mathrm{MHz}\right) \delta_{\mathrm{H}} 8.50(\mathrm{~d}, J=2.0 \mathrm{~Hz}, \mathrm{H}-8), 8.0$ (dd, $J=2.0,8.5 \mathrm{~Hz}, \mathrm{H}-4), 7.10$ (d, $J=15.0 \mathrm{~Hz}, \mathrm{H}-1), 7.00$ (d, $J=8.5 \mathrm{~Hz}, \mathrm{H}-5), 5.53$ (d, $J=15.0 \mathrm{~Hz}, \mathrm{H}-2), 3.87$ (s, OMe), 3.70 (s, OMe); ESIMS $m / z 242.99[\mathrm{M}+\mathrm{H}]^{+}$.

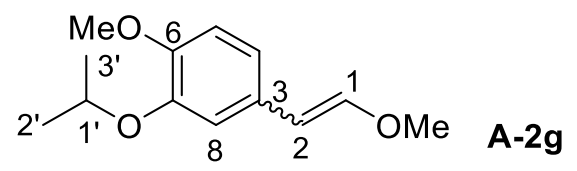

$\boldsymbol{A}-\boldsymbol{2 g}\left(\right.$ yield 75\%): ${ }^{1} \mathrm{H} \mathrm{NMR}\left(\mathrm{CDCl}_{3}, 400 \mathrm{MHz}\right) \delta_{\mathrm{H}} 7.25(\mathrm{~d}, J=2.0 \mathrm{~Hz}, \mathrm{H}-8), 7.50$ (dd, $J=2.0,8.5 \mathrm{~Hz}, \mathrm{H}-4), 6.60$ (d, $J=15.0 \mathrm{~Hz}, \mathrm{H}-1), 7.05$ (d, $J=8.5 \mathrm{~Hz}, \mathrm{H}-5), 5.40$ (d, $J=15.0 \mathrm{~Hz}, \mathrm{H}-2), 3.85$ (s, OMe), 3.75 (s, OMe), 4.40 (m, H-1'), 1.30 (d, $J=6.0$ $\mathrm{Hz}, \mathrm{H}_{3}-2^{\prime}$ ), 1.32 (d, $J=6.0 \mathrm{~Hz}, \mathrm{H}_{3}-3$ '); ESIMS $m / z 223.28[\mathrm{M}+\mathrm{H}]^{+}$.<smiles>[R3]c1ccc(/C=C/OC)cc1[R]</smiles>

A-2d. $\mathrm{R}_{3}=\mathrm{OMe}, \mathrm{R}_{4}=\mathrm{H}$

A-2e. $\mathrm{R}_{3}=\mathrm{OMe}, \mathrm{R}_{4}=\mathrm{Cl}$

A-2f. $\mathrm{R}_{3}=\mathrm{OMe}, \mathrm{R}_{4}=\mathrm{Br}$

A-2g. $\mathrm{R}_{3}=\mathrm{OMe}, \mathrm{R}_{4}=\mathrm{OiPr}$<smiles>[R]c1ccc(CC=O)cc1[R]</smiles>

A-0c. $R_{3}=R_{4}=H$ A-0d. $\mathrm{R}_{3}=\mathrm{OMe}, \mathrm{R}_{4}=\mathrm{H}$ A-Oe. $R_{3}=\mathrm{OMe}, \mathrm{R}_{4}=\mathrm{Cl}$ A-Of. $\mathrm{R}_{3}=\mathrm{OMe}, \mathrm{R}_{4}=\mathrm{Br}$ $A-0 g \cdot R_{3}=\mathrm{OMe}, \mathrm{R}_{4}=\mathrm{OiPr}$

Scheme S3. Synthesis of A-0c to A-0g.

Synthesis of A-0d to A-0g: A-0c is a commercial product. To a mixture of A-2d (1.0 eq.) in tetrahydrofuran, $\mathrm{HCl}(2 \mathrm{~N})$ was added to reflux for $3 \mathrm{~h}$. After cooling to rt, the $\mathrm{pH}$ was adjusted to 6-7 by saturated $\mathrm{Na}_{2} \mathrm{CO}_{3}$. The mixture was then extracted with EtOAc and concentrated under vacuo to yield A-0d with 93\% yield. Following the same protocol as for A-0d, A-0e (yield 97\%), A-0f (yield 95\%) and A-0g (yield 44\%) were derived from A-2e, A-2f and A-2g respectively. 


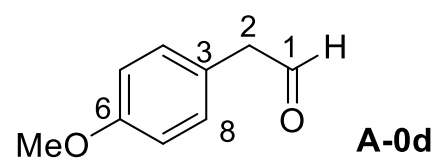

A-0d (2-(4-methoxyphenyl)acetaldehyde, 68\% yield): Light yellow liquid, ${ }^{1} \mathrm{H}$ NMR (400 MHz, $\left.\mathrm{CDCl}_{3}\right) \delta_{\mathrm{H}} 9.70(\mathrm{t}, J=2.4 \mathrm{~Hz}, \mathrm{H}-1), 7.14$ (d, $\left.J=8.5 \mathrm{~Hz}, \mathrm{H}-4 / \mathrm{H}-8\right)$, $6.90(\mathrm{~d}, J=8.5 \mathrm{~Hz}, \mathrm{H}-5 / \mathrm{H}-7), 3.80$ (s, OMe), 3.62 (d, $\left.J=2.4 \mathrm{~Hz}, \mathrm{H}_{2}-2\right) .{ }^{13} \mathrm{C} \mathrm{NMR}$ $\left(100 \mathrm{MHz}, \mathrm{CDCl}_{3}\right) \delta_{\mathrm{C}} 199.7$ (C-1), 159.0 (C-6), 130.7 (C-4/C-8), 123.7 (C-3), 114.5 (C-5/C-7), 55.3 (OMe), 49.7 (C-2). ESIMS m/z $151.08[\mathrm{M}+\mathrm{H}]^{+}$.

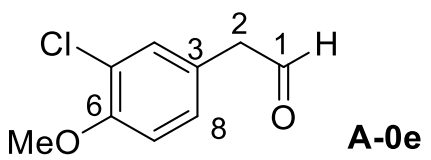

A-0e (2-(3-chloro-4-methoxyphenyl)acetaldehyde): Colorless oil, ${ }^{1} \mathrm{H}$ NMR $\left(\mathrm{CDCl}_{3}, 400 \mathrm{MHz}\right) \delta_{\mathrm{H}} 9.72(\mathrm{t}, J=2.2 \mathrm{~Hz}, \mathrm{H}-1), 7.23$ (d, $\left.J=2.2 \mathrm{~Hz}, \mathrm{H}-4\right), 7.07$ (dd, $J$ $=2.2,8.4 \mathrm{~Hz}, \mathrm{H}-8), 6.92(\mathrm{~d}, J=8.4 \mathrm{~Hz}, \mathrm{H}-7), 3.90(\mathrm{~s}, \mathrm{OMe}), 3.62(\mathrm{~d}, J=2.2 \mathrm{~Hz}$, $\left.\mathrm{H}_{2}-2\right) ;{ }^{13} \mathrm{C}$ NMR $\left(\mathrm{CDCl}_{3}, 100 \mathrm{MHz}\right) \delta_{\mathrm{C}} 199.0(\mathrm{C}-1), 154.4(\mathrm{C}-6), 131.3$ (C-8), 129.0 (C-4), 124.8 (C-3), 122.8 (C-5), 112.5 (C-7), 56.2 (OMe), 49.2 (C-2); HRESIMS m/z $183.0270[\mathrm{M}-\mathrm{H}]^{-}$(calcd for $\mathrm{C}_{9} \mathrm{H}_{8} \mathrm{ClO}_{2}$ 183.0291).

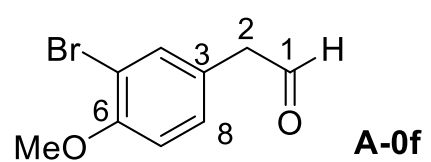

A-0f (2-(3-bromo-4-methoxyphenyl)acetaldehyde): Colorless oil, ${ }^{1} \mathrm{H}$ NMR $\left(\mathrm{CDCl}_{3}, 400 \mathrm{MHz}\right) \delta_{\mathrm{H}} 9.71(\mathrm{t}, J=2.0 \mathrm{~Hz}, \mathrm{H}-1), 7.40$ (d, $\left.J=2.0 \mathrm{~Hz}, \mathrm{H}-4\right), 7.11(\mathrm{dd}, J=$ 2.0, 8.4 Hz, H-8), 6.89 (d, J=8.4 Hz, H-7), 3.88 (s, OMe), 3.61 (d, J=2.0 Hz, H2-2); ${ }^{13} \mathrm{C} \mathrm{NMR}\left(\mathrm{CDCl}_{3}, 100 \mathrm{MHz}\right) \delta_{\mathrm{C}} 198.9$ (C-1), 155.4 (C-6), 134.5 (C-3), 129.8 (C-8), 125.4 (C-4), 112.4 (C-7), 112.2 (C-5), 56.4 (OMe), 49.2 (C-2). ESIMS m/z 228.97 $[\mathrm{M}+\mathrm{H}]^{+}$.<smiles>COc1ccc(CC=O)cc1O[14CH3]</smiles>

A-0g (2-(3-isopropoxy-4-methoxyphenyl)acetaldehyde): Colorless oil, IR $v_{\max }$ 2976, 1683, $1258 \mathrm{~cm}^{-1} ;{ }^{1} \mathrm{H} \mathrm{NMR}\left(\mathrm{CDCl}_{3}, 400 \mathrm{MHz}\right) \delta_{\mathrm{H}} 9.73(\mathrm{t}, J=1.5 \mathrm{~Hz}, \mathrm{H}-1), 6.89$ 
$(\mathrm{d}, J=8.0 \mathrm{~Hz}, \mathrm{H}-7), 6.78$ (dd, $J=2.0,8.0 \mathrm{~Hz}, \mathrm{H}-8), 6.77$ (dd, $J=2.0 \mathrm{~Hz}, \mathrm{H}-4), 4.57$ (m, H-1'), 3.86 (s, OMe), 3.61 (d, $\left.J=1.5 \mathrm{~Hz}, \mathrm{H}_{2}-2\right), 1.38$ (d, $J=6.1 \mathrm{~Hz}, \mathrm{H}_{3}-2^{\prime} / \mathrm{H}_{3}-3^{\prime}$ ); ${ }^{13} \mathrm{C}$ NMR $\left(\mathrm{CDCl}_{3}, 100 \mathrm{MHz}\right) \delta_{\mathrm{C}} 199.6$ (C-1), 149.9 (C-5), 147.7 (C-6), 124.1 (C-3), 122.4 (C-8), 117.3 (C-4), 112.5 (C-7), 71.6 (C-1'), 56.1 (OMe), 50.1 (C-2), 22.2 (C-2'/C-3'). ESIMS m/z 209.11 [M+H] .
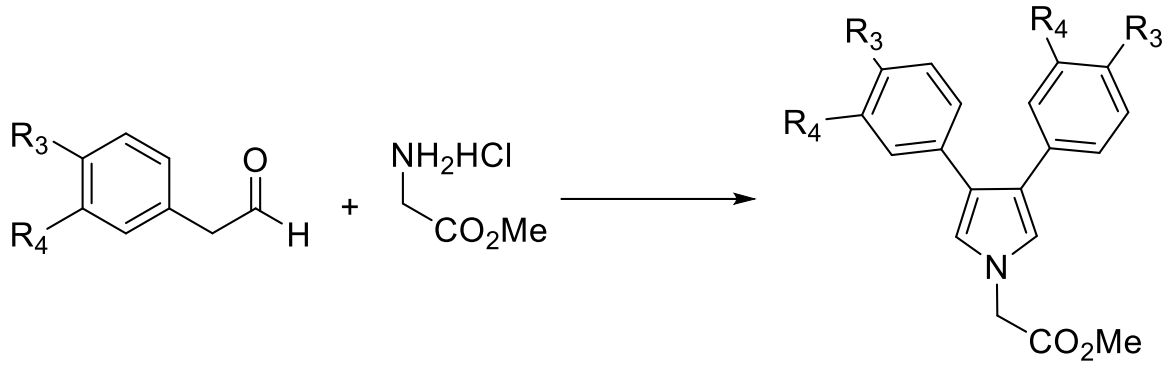

$$
\begin{aligned}
& \text { A-0. } R_{3}=R_{4}=O M e \\
& \text { A-0c. } R_{3}=R_{4}=H \\
& \text { A-0d. } R_{3}=O M e, R_{4}=H \\
& \text { A-0e. } R_{3}=O M e, R_{4}=C l \\
& \text { A-0f. } R_{3}=O M e, R_{4}=B r \\
& \text { A-0g. } R_{3}=O M e, R_{4}=O i P r
\end{aligned}
$$

$$
\begin{aligned}
& \text { 1. } R 3=R 4=O M e \\
& \text { 1c. } R 3=R 4=H \\
& \text { 1d. } R 3=O M e, R 4=H \\
& \text { 1e. } R 3=O M e, R 4=C l \\
& \text { 1f. } R 3=O M e, R 4=B r \\
& \text { 1g. } R 3=O M e, R 4=O i P r
\end{aligned}
$$

\section{Scheme S4. Synthesis of 1 and 1c-1g}

Synthesis of 1 and 1c-1g: To a solution of A-0 (1.0 eq.) in tetrahydrofuran, methyl-2-aminoacetatehydrochloride (1.0 eq.) was added under $\mathrm{N}_{2}$. After stirring at $\mathrm{rt}$ for 30 min, $\mathrm{AgOAc}(3.0$ eq.) and $\mathrm{NaOAc}$ (3.0 eq.) were added. The mixture was refluxed for $16 \mathrm{~h}$. After cooling to $\mathrm{rt}$, the solid was purified by silica gel column eluting by $\mathrm{CH}_{2} \mathrm{Cl}_{2}-\mathrm{MeOH}$ (3:1) to yield 1 (yield 11\%). 1c-1g with the yield of 47\%, $10 \%, 12 \%, 18 \%$ and $11 \%$ respectively were obtained from A-0c to A-0g respectively by the same protocol as for $\mathbf{1}$.

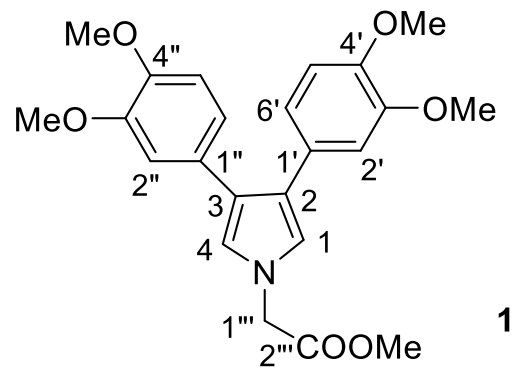

1 (methyl-2-(3,4-bis(3,4-dimethoxyphenyl)-1H-pyrrol-1-yl)acetate): Light yellow solid, IR $v_{\max } 2937,1730,1226 \mathrm{~cm}^{-1} ;{ }^{1} \mathrm{H} \mathrm{NMR}\left(\mathrm{CDCl}_{3}, 400 \mathrm{MHz}\right) \delta_{\mathrm{H}} 6.85(\mathrm{~s}$, H-1/H-4), 6.79 (dd, $J=2.0,8.0$ Hz, H-6'/H-6”), 6.76 (d, J=2.0 Hz, H-2'/H-2”), 6.75 
6.75 (d, $J=8.0 \mathrm{~Hz}, \mathrm{H}-5$ '/H-5"), 4.67 (s, H2-1"'), 3.86 (s, OMe/OMe), 3.81 (s, OMe), 3.68 (s, OMe/OMe); ${ }^{13} \mathrm{C}$ NMR $\left(\mathrm{CDCl}_{3}, 100 \mathrm{MHz}\right) \delta_{\mathrm{C}} 169.1$ (C-2"”), 148.5 (C-4'/C-4”), 147.4 (C-3'/C-3”), 128.5 (C-1'/C-1”), $124.1 \quad$ (C-1/C-4), 120.8 (C-6'/C-6”), 120.7 (C-2'/C-2”), 112.3 (C-3'/C-3”), 111.2 (C-2/C-3), 56.0 (OMe×2), 55.8 (OMe×2), 52.8 (C-1'”), $51.0(\mathrm{OMe})$; HRESIMS m/z 412.1770 [M+H] ${ }^{+}$(calcd for $\mathrm{C}_{23} \mathrm{H}_{26} \mathrm{NO}_{6} 412.1682$ ).

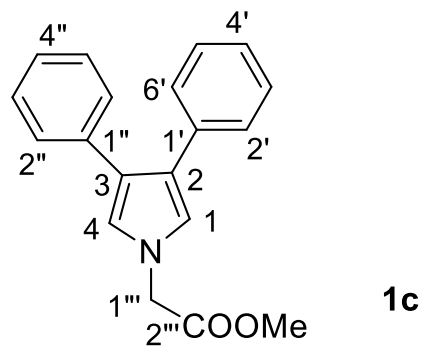

1c (methyl-2-(3,4-diphenyl-1H-pyrrol-1-yl)acetate): Light yellow solid, IR $v_{\max } 2951,1751 \mathrm{~cm}^{-1} ;{ }^{1} \mathrm{H} \quad \mathrm{NMR} \quad\left(\mathrm{CDCl}_{3}, 400 \mathrm{MHz}\right) \delta_{\mathrm{H}}$ 7.29-7.23 (m, H-2'/H-6'/H-2”/H-6”, H3'/H-5'/H-3”/H-5”), 7.21 (m, H-4'/H-4”), 6.79 (s, H-1/H-4), 4.68 (s, $\mathrm{H}_{2}-1 "$ "), 3.81 (s, OMe); ${ }^{13} \mathrm{C}$ NMR $\left(\mathrm{CDCl}_{3}, 100 \mathrm{MHz}\right) \delta_{\mathrm{C}} 169.0$ (C-2"”), 135.6 (C-1'/C-1”), 128.6 (C-2'/C-6'/C-2”/C-6”), 128.3 (C-3'/C-5'/C-3”/C-5”), 125.9 (C-4'/C-4"), 124.4 (C-1/C-4), 121.4 (C-2/C-3), 52.8 (C-1"'), 51.0 (OMe); HRESIMS $m / z 292.1338[\mathrm{M}+\mathrm{H}]^{+}\left(\right.$calcd for $\left.\mathrm{C}_{19} \mathrm{H}_{18} \mathrm{NO}_{2} 292.1259\right)$.

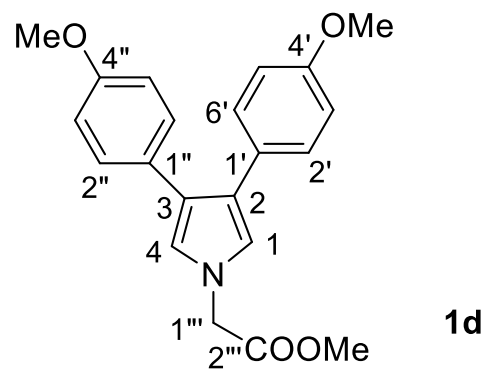

1d (methyl-2-(3,4-bis(4-methoxyphenyl)-1H-pyrrol-1-yl)acetate): Light yellow solid, IR $v_{\max } 2952,1748,1249 \mathrm{~cm}^{-1} ;{ }^{1} \mathrm{H}$ NMR $\left(\mathrm{CDCl}_{3}, 400 \mathrm{MHz}\right) \delta_{\mathrm{H}} 7.20(\mathrm{~d}, J=8.0$ Hz, H-2'/H-6'/H-2"/H-6”), 6.82 (d, $J=8.0 \mathrm{~Hz}, \mathrm{H}-3$ '/H-5'/H-3”/H-5”), 6.71 (s, $\mathrm{H}-1 / \mathrm{H}-4), 4.65$ (s, $\mathrm{H}_{2}-1$ "'), 3.80 (s, OMe), 3.79 (s, OMe $\left.\times 2\right) ;{ }^{13} \mathrm{C} \mathrm{NMR}\left(\mathrm{CDCl}_{3}, 100\right.$ MHz) $\delta 169.1,157.9,129.6,128.2,123.8,120.7,113.7,55.3,52.7,50.9$; HRESIMS $m / z 352.1541[\mathrm{M}+\mathrm{H}]^{+}$(calcd for $\mathrm{C}_{21} \mathrm{H}_{22} \mathrm{NO}_{4} 352.1471$ ). 


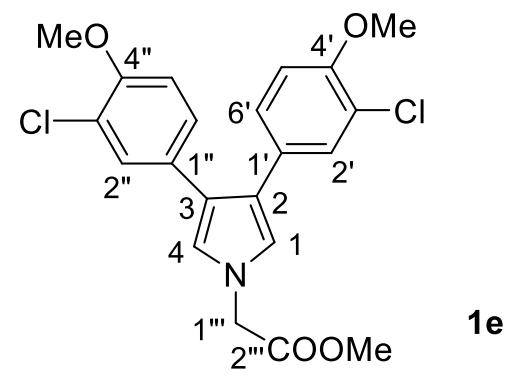

1e (methyl-2-(3,4-bis(3-chloro-4-methoxyphenyl)-1H-pyrrol-1-yl)acetate): Light yellow solid, IR $v_{\max } 2930,1748,1259 \mathrm{~cm}^{-1} ;{ }^{1} \mathrm{H} \mathrm{NMR}\left(\mathrm{CDCl}_{3}, 400 \mathrm{MHz}\right) \delta_{\mathrm{H}}$ 7.30 (d, $J=2.1 \mathrm{~Hz}, \mathrm{H}-2$ '/H-2”), 7.03 (dd, $J=2.1,8.5$ Hz, H-6'/H-6”), 6.80 (d, $J=8.5$ Hz, H-5'/H-5"), 6.71 (s, H-1/H-4), 4.64 (s, H2-1"'), 3.88 (s, OMe×2), 3.80 (s, OMe); ${ }^{13} \mathrm{C}$ NMR $\left(\mathrm{CDCl}_{3}, 100 \mathrm{MHz}\right) \delta_{\mathrm{C}} 168.9$ (C-2"”), 153.4 (C-4'/C-4”), 130.0 (C-1'/C-1”), 128.9 (C-1/C-4), 127.9 (C-6'/C-6”), 122.6 (C-2'/C-2”), 122.1 (C-3'C-3”), 121.1 (C-2/C-3), 112.0 (C-5'/C-5”), 56.2 (OMe×2), 52.8 (C-1"'), 50.9 (OMe); HRESIMS $m / z 420.0768[\mathrm{M}+\mathrm{H}]^{+}$(calcd for $\mathrm{C}_{21} \mathrm{H}_{20} \mathrm{Cl}_{2} \mathrm{NO}_{4} 420.0691$ ).

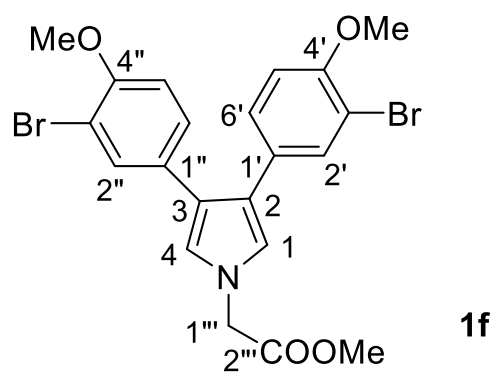

1f (methyl-2-(3,4-bis(3-bromo-4-methoxyphenyl)-1H-pyrrol-1-yl)acetate):

Light yellow solid, IR $v_{\max } 2918,1747,1250 \mathrm{~cm}^{-1} ;{ }^{1} \mathrm{H} \mathrm{NMR}\left(\mathrm{CDCl}_{3}, 400 \mathrm{MHz}\right) \delta_{\mathrm{H}}$ 7.49 (d, $J=2.1 \mathrm{~Hz}, \mathrm{H}-2 / \mathrm{H}-2 ”), 7.06$ (dd, $J=2.1,8.5$ Hz, H-6'/H-6”), 6.77 (d, $J=8.5$ Hz, H-5'/H-5”), 6.71 (s, H-1/H-4), 4.64 (s, H2-1"”), 3.87 (s, OMe×2), 3.80 (s, OMe); ${ }^{13} \mathrm{C}$ NMR $\left(\mathrm{CDCl}_{3}, 100 \mathrm{MHz}\right) \delta_{\mathrm{C}} 169.0$ (C-2”'), 154.2 (C-4'/C-4”), 133.0 (C-1'/C-1”), 129.4 (C-6'/C-6”), 128.7 (C-1/C-4), 122.5 (C-6'/C-6”), 121.1 (C-3'/C-3”), 111.8 (C-5'/C-5”), 111.4 (C-2/C-3), 56.3 (OMe×2), 52.8 (C-1"'), 50.9 (OMe); HRESIMS $m / z 507.9759[\mathrm{M}+\mathrm{H}]^{+}$(calcd for $\mathrm{C}_{21} \mathrm{H}_{20} \mathrm{Br}_{2} \mathrm{NO}_{4}$ 507.9681). 


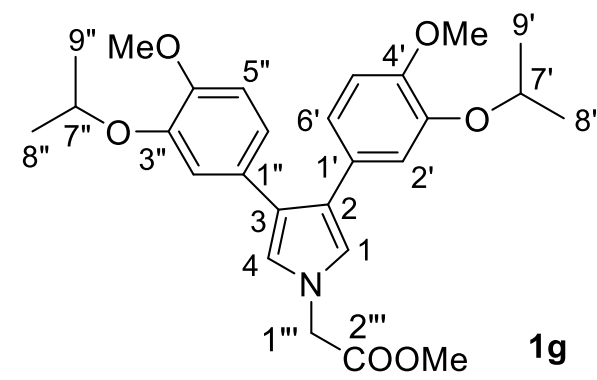

$1 \mathrm{~g}$ (methyl-2-(3,4-bis(3-isopropoxy-4-methoxyphenyl)-1H-pyrrol-1-yl)acetate): Light yellow solid, IR $v_{\max } 2975,1748,1254 \mathrm{~cm}^{-1} ;{ }^{1} \mathrm{H} \mathrm{NMR}\left(\mathrm{CDCl}_{3}, 400 \mathrm{MHz}\right) \delta_{\mathrm{H}}$ 6.84 (d, $J=2.0 \mathrm{~Hz}, \mathrm{H}-2$ '/H-2”), 6.82 (dd, $J=2.0,8.0 \mathrm{~Hz}, \mathrm{H}-6$ '/H-6”), 6.77 (d, $J=8.0$ Hz, H-5'/H-5”), 6.71 (s, H-1/H-4), 4.64 (s, H2-1”'), 4.32 (m, H-7'/H-7”), 3.81 (s, OMe $\times 2$ ), 3.79 (s, OMe), 1.22 (d, $\left.J=6.1 \mathrm{~Hz}, \mathrm{H}_{3}-8^{\prime} / \mathrm{H}_{3}-9^{\prime} / \mathrm{H}_{3}-88^{\prime \prime} / \mathrm{H}_{3}-9^{\prime \prime}\right) ;{ }^{13} \mathrm{C} \mathrm{NMR}$ $\left(\mathrm{CDCl}_{3}, 100 \mathrm{MHz}\right) \delta_{\mathrm{C}} 169.1$ (C-2”’), 148.7 (C-3'/C-3”), 147.0 (C-4'/C-4”), 128.6 (C-1'/C-1”), $124.1 \quad$ (C-1/C-4), $121.0 \quad$ (C-6'/C-6”), $120.6 \quad$ (C-2'/C-2”), 116.6 (C-5'/C-5”), 112.1 (C-2/C-3), 71.1 (C-7'/C-7’), 56.1 (OMe×2), 52.6 (C-1"”), 50.9 (OMe), 22.1 (C-8'/C-9'/C-8"/C-9"); HRESIMS m/z $468.2389[\mathrm{M}+\mathrm{H}]^{+}$(calcd for $\left.\mathrm{C}_{27} \mathrm{H}_{34} \mathrm{NO}_{6} 468.2308\right)$.
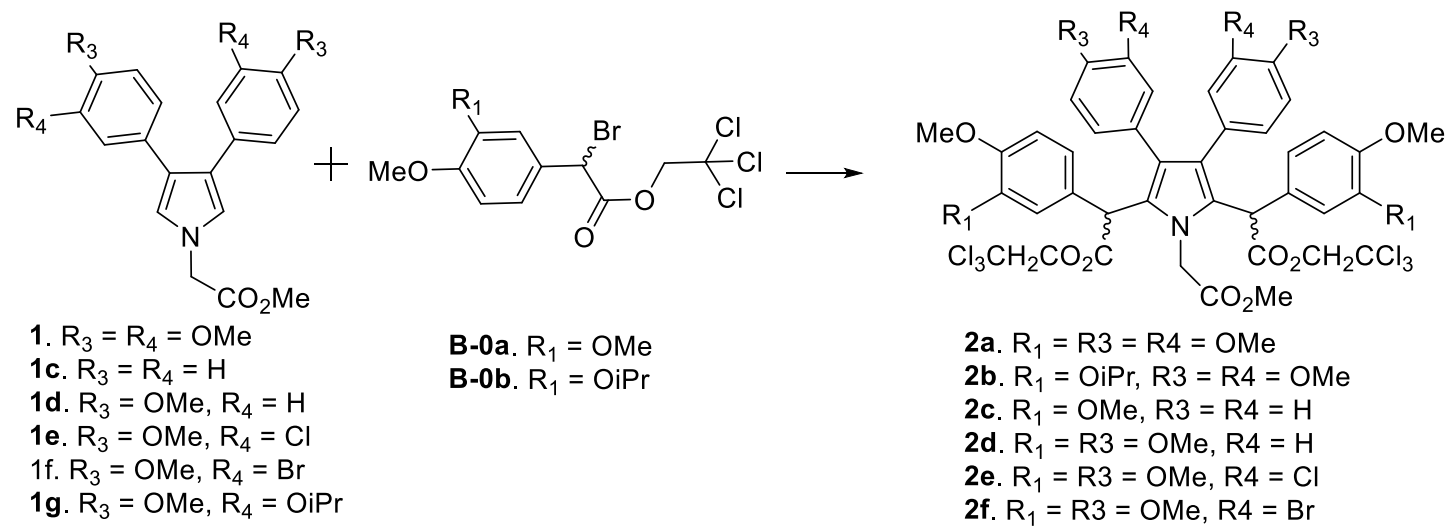

$$
\begin{aligned}
& \text { 2a. } R_{1}=R 3=R 4=O M e \\
& \text { 2b. } R_{1}=O i P r, R 3=R 4=O M e \\
& \text { 2c. } R_{1}=O M e, R 3=R 4=H \\
& \text { 2d. } R_{1}=R 3=O M e, R 4=H \\
& \text { 2e. } R_{1}=R 3=O M e, R 4=C l \\
& \text { 2f. } R_{1}=R 3=O M e, R 4=B r \\
& \text { 2g. } R_{1}=R 3=O M e, R 4=O i P r
\end{aligned}
$$

\section{Scheme S5. Synthesis of 2 a to $2 \mathrm{~g}$}

Synthesis of 2 a to $2 \mathrm{~g}$ : $\mathbf{B - 0 a}\left(2.5\right.$ eq.) in dry $\mathrm{CH}_{2} \mathrm{Cl}_{2}(10 \mathrm{~mL})$ was added to a mixture of 1 (1.0 eq.) and $\mathrm{Al}_{2} \mathrm{O}_{3}$ (30.0 eq.) in dry $\mathrm{CH}_{2} \mathrm{Cl}_{2}$ under $\mathrm{N}_{2}$ to stir at $30{ }^{\circ} \mathrm{C}$ for $20 \mathrm{~h}$. The solid was filtered and washed with $\mathrm{CH}_{2} \mathrm{Cl}_{2}$, while the concentrated filtrate was purified by silica gel column eluting with $\mathrm{CH}_{2} \mathrm{Cl}_{2}-\mathrm{MeOH}(3: 1)$ to yield 2a (yield 40\%). Analogues 2b (yield 38\%), 2c (yield 48\%), 2d (yield 68\%), 2e (yield 75\%), 2f (yield 35\%) and $\mathbf{2 g}$ (yield 60\%) were generated from 1c-1g with B-0a or B-0b following the same protocol as for $\mathbf{2 a}$. 


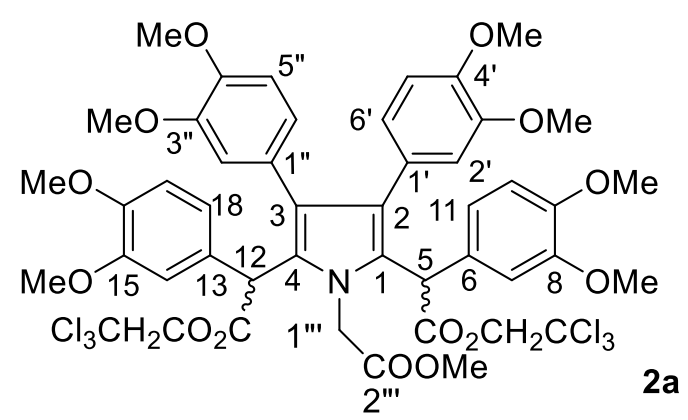

2a (bis(2,2,2-trichloroethyl)2,2'-(3,4-bis(3,4-dimethoxyphenyl)-1-(2-methoxy -2-oxoethyl)-1H-pyrrole-2,5-diyl)bis(2-(3,4-dimethoxyphenyl)acetate): Light yellow solid, IR $v_{\max } 2931,1745,1248 \mathrm{~cm}^{-1} ;{ }^{1} \mathrm{H} \mathrm{NMR}\left(\mathrm{CDCl}_{3}, 400 \mathrm{MHz}\right) \delta_{\mathrm{H}} 6.91$ (dd, $J=2.0,7.5$ Hz, H-11/H-18), 6.90 (dd, $J=2.0,8.0$ Hz, H-6'/H-6”), 6.75 (d, $J=2.0$ Hz, H-7/H-14), 6.72 (d, J=8.0, Hz, H-10/H-17), 6.65 (d, J=2.0 Hz, H-2'/H-2”), 6.57 (d, $J=8.0 \mathrm{~Hz}, \mathrm{H}-5$ '/H-5”), 5.52 (s, H-5/H-12), 4.87 (s, $\mathrm{H}_{2}-1$ '”), 4.66 (d, J=12.0 Hz, 2H), $4.47(\mathrm{~d}, J=12.0 \mathrm{~Hz}, 2 \mathrm{H}), 3.83(\mathrm{~s}, \mathrm{OMe} \times 2), 3.79$ (s, OMe $\times 2), 3.59$ (s, OMe $\times 4), 3.32$ (s, OMe); ${ }^{13} \mathrm{C} \mathrm{NMR}\left(\mathrm{CDCl}_{3}, 100 \mathrm{MHz}\right) \delta_{\mathrm{C}} 170.5$ (C-2"”), $168.8(\mathrm{CO} \times 2), 149.5$ (C-8/C-15), 148.4 (C-4'/C-4”), 147.7 (C-9/C-16), 147.2 (C-3'/C-3”), 127.8 (C-1'/C-1”), 127.7 (C-6/C-13), 125.9 (C-1/C-4), 125.9 (C-11/C-18), 123.1 (C-6'/C-6”), 120.5 (C-2'/C-2”), 115.9 (C-7/C-14), 114.5 (C-5'/C-5”), 111.6 (C-10/C-19), $110.8(\mathrm{C}-2 / \mathrm{C}-3), 94.9\left(\mathrm{CCl}_{3} \times 2\right), 71.4\left(\mathrm{CH}_{2} \mathrm{O} \times 2\right), 56.0(\mathrm{OMe} \times 2), 55.9$ (OMe×4), 55.9 (OMe×2), $51.8(\mathrm{OMe}), 47.9$ (C-1"”), $46.9\left(\mathrm{CH}_{2} \mathrm{O} \times 2\right)$; HRESIMS m/z $1060.1178[\mathrm{M}+\mathrm{H}]^{+}$(calcd for $\left.\mathrm{C}_{47} \mathrm{H}_{47} \mathrm{Cl}_{6} \mathrm{NO}_{14}\right)$.

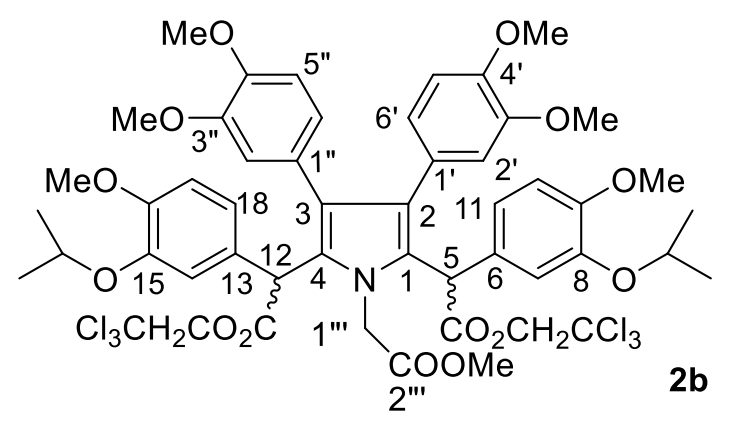

2b (bis(2,2,2-trichloroethyl)2,2'-(3,4-bis(3,4-dimethoxyphenyl)-1-(2-methoxy -2-oxoethyl)-1H-pyrrole-2,5-diyl)bis(2-(3-isopropoxy-4-methoxyphenyl)acetate):

Light yellow solid, IR $v_{\max } 2933,1748,1251 \mathrm{~cm}^{-1} ;{ }^{1} \mathrm{H} \mathrm{NMR}\left(\mathrm{CDCl}_{3}, 400 \mathrm{MHz}\right) \delta_{\mathrm{H}}$ 6.91 (d, $J=7.6$ Hz, H-10/H-17), 6.75 (dd, $J=7.5,2.0$ Hz, H-6'/H-6”), 6.72 (d, $J=2.0$ Hz, H-2'/H-2”), 6.65 (d, J=2.0 Hz, H-7/H-14), 6.62 (d, $J=7.5$ Hz, H-10/H-17), 6.57 
(d, $J=2.0$ Hz, H-5'/H-5”), 5.52 (s, H-5/H-12), 4.87 (s, $\mathrm{H}_{2}-1$ "'), 4.82 (m, iPr×2), 4.66 (d, $\left.J=11.9 \mathrm{~Hz}, \mathrm{OCH}_{2}\right), 4.47$ (d, $\left.J=11.9 \mathrm{~Hz}, \mathrm{OCH}_{2}\right), 3.83$ (s, OMe×2), 3.79 (s, $\mathrm{OMe} \times 2), 3.59$ (s, OMe $\times 2), 3.32$ (s, OMe), 1.29 (m, iPr-Me×4); ${ }^{13} \mathrm{C} \mathrm{NMR}\left(\mathrm{CDCl}_{3}\right.$, $100 \mathrm{MHz}) \delta_{\mathrm{C}} 170.5$ (C-2””), 168.8 (CO×2), 149.5 (C-8/C-15), 148.4 (C-4'/C-4”), 147.7 (C-9/C-16), 147.2 (C-3'/C-3”), 127.8 (C-1/C-4), 127.7 (C-6/C-13), 125.9 (C-1'/C-1"), 125.9 (C-11/C-18), 123.1 (C-6'/C-6”), 120.5 (C-7/C-15), 115.9 (C-10/C-17), 114.5 (C-2'/C-2”), 111.6 (C-5'/C-5”), 110.8 (C-2/C-3), $94.9\left(\mathrm{CCl}_{3} \times 2\right)$, 74.6 (iPr×2), $71.4\left(\mathrm{OCH}_{2} \times 2\right), 56.0(\mathrm{OMe} \times 2), 55.9(\mathrm{OMe} \times 2), 55.9(\mathrm{OMe} \times 2), 51.8$ (OMe), 47.9 (C-1"'), 46.9 (C-2/C-5), 22.1 (Me×4); HRESIMS m/z 1118.2198 [M+H] ${ }^{+}$ (calcd for $\mathrm{C}_{51} \mathrm{H}_{56} \mathrm{Cl}_{6} \mathrm{NO}_{14} 1118.1724$ ).

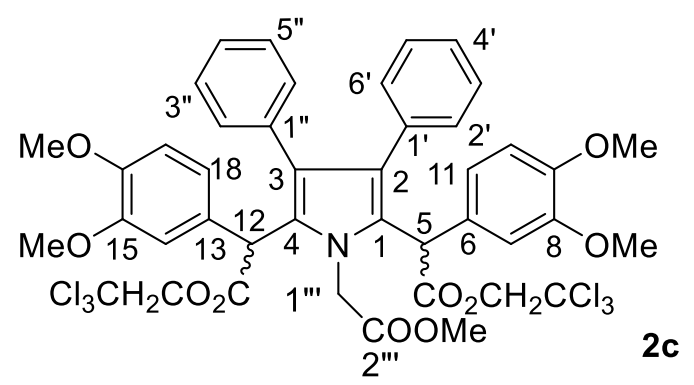

2c (bis(2,2,2-trichloroethyl)2,2'-(1-(2-methoxy-2-oxoethyl)-3,4-diphenyl-1Hpyrrole-2,5-diyl)bis(2-(3,4-dimethoxyphenyl)acetate): Light yellow solid, IR $v$ 2951, 1751, $1251 \mathrm{~cm}^{-1} ;{ }^{1} \mathrm{H}$ NMR $\left(\mathrm{CDCl}_{3}, 400 \mathrm{MHz}\right) \delta 7.24-7.14$ (m, 10H), 6.78-6.71 (m, 4H), 6.66 (s, 2H), 5.45 (s, 2H), 4.82 (d, J=11.9 Hz, 2H), 4.57 (d, J = 11.9 Hz, 2H), $4.47(\mathrm{~s}, 2 \mathrm{H}), 3.86(\mathrm{~s}, 6 \mathrm{H}), 3.76(\mathrm{~s}, 6 \mathrm{H}), 3.58(\mathrm{~s}, 3 \mathrm{H}) ;{ }^{13} \mathrm{C} \mathrm{NMR}\left(\mathrm{CDCl}_{3}, 100\right.$ MHz) $\delta 170.1,169.6,149.1,148.4,134.9,130.9,128.2$, 128.0, 126.9, 126.6, 125.8, 120.1, 111.7, 111.1, 94.9, 74.7, 56.0, 55.9, 52.3, 47.9, 46.5; HRESIMS $m / z$ 940.0786 $[\mathrm{M}+\mathrm{H}]^{+}$(calcd for $\left.\mathrm{C}_{43} \mathrm{H}_{40} \mathrm{Cl}_{6} \mathrm{NO}_{10} 940.0705\right)$.

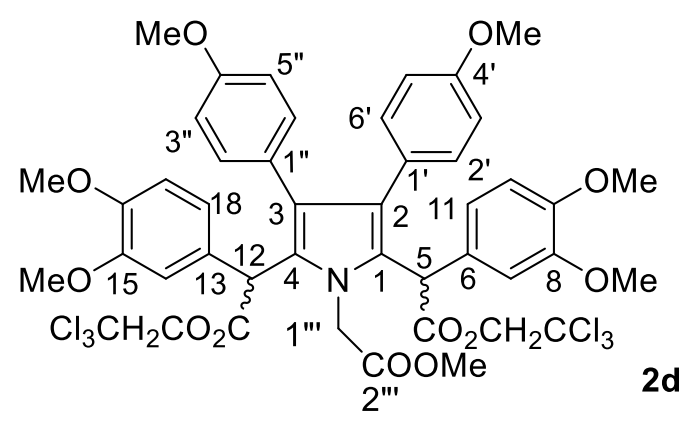




\section{methoxyphenyl)-1H-pyrrole-2,5-diyl)-bis(2-(3,4-dimethoxyphenyl)acetate):}

Yellow solid, IR $v_{\max } 2918,1750,1242 \mathrm{~cm}^{-1} ;{ }^{1} \mathrm{H}$ NMR $\left(\mathrm{CDCl}_{3}, 400 \mathrm{MHz}\right) \delta_{\mathrm{H}} 7.21$ (dd, $J=8.0,2.0$ Hz, H-11/H-18), 7.13 (d, $J=8.0$ Hz, H-2'/H-6'/2"/H-6”), 6.78 (d, $J$ $=2.0 \mathrm{~Hz}, \mathrm{H}-7 / \mathrm{H}-14), 6.76(\mathrm{~d}, J=2.0 \mathrm{~Hz}, \mathrm{H}-7 / \mathrm{H}-14), 6.52(\mathrm{~d}, J=8.0 \mathrm{~Hz}$, H-3'/H-5'/3"/H-3”), 5.48 (s, H-5/H-12), 5.43 (s, H2-1"'), 4.87 (m, $\left.\mathrm{OCH}_{2} \times 2\right), 3.85$ (OMe×2), $3.74(\mathrm{OMe} \times 2), 3.58(\mathrm{OMe} \times 2), 3.30(\mathrm{~s}, \mathrm{OMe}) ;{ }^{13} \mathrm{C} \mathrm{NMR}\left(\mathrm{CDCl}_{3}, 100\right.$ MHz) $\delta_{\mathrm{C}} 170.6 / 170.2$ (C-2"”), 169.7/168.9 (C=O), 158.3/158.2 (C-4'/C-4”), 149.0 (C-8/C-15), 148.8/148.4 (C-9/C-16), 132.1/131.9 (C-1'/C-1"), 128.3 (C-2'/C-6'/C-2"/C-6”), $128.0 \quad$ (C-6/C-13), $127.3 \quad$ (C-1'/C-1"), 126.5/125.9 (C-11/C-18), $\quad 125.8 / 125.5 \quad$ (C-1/C-4), $120.1 / 120.0 \quad$ (C-7/C-14), 113.5/113.5 (C-10/C-17), 111.7/111.6 (C-3'/C-5'/C-3"/C-5”), 111.0/110.9 (C-2/C-3), 95.0/94.9 $\left(\mathrm{CCl}_{3} \times 2\right)$, 74.4/74.7 $\left(\mathrm{OCH}_{2} \times 2\right), 56.0 / 55.9(\mathrm{OMe} \times 2), 55.9 / 55.8(\mathrm{OMe} \times 2), 55.2 / 55.2$ (OMe×2), 52.2/51.7 (OMe), , 48.0/46.9 (C-1"”), $46.5\left(\mathrm{OCH}_{2} \times 2\right)$; HRESIMS m/z 1000.0956 [M+H] $]^{+}$(calcd for $\mathrm{C}_{45} \mathrm{H}_{44} \mathrm{Cl}_{6} \mathrm{NO}_{12}$ 1000.0916).

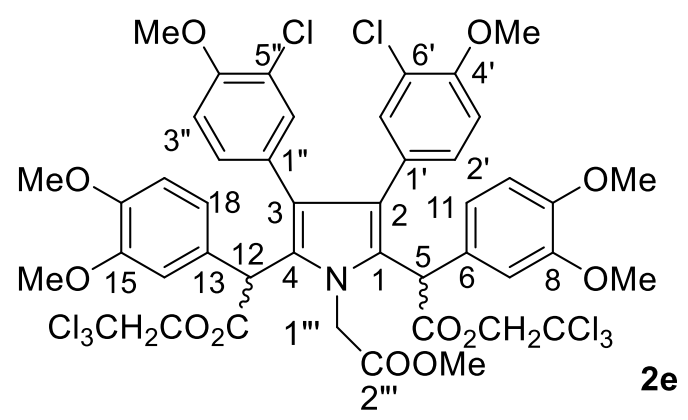

2e bis(2,2,2-trichloroethyl)2,2'-(3,4-bis(3-chloro-4-methoxyphenyl)-1-(2methoxy-2-oxoethyl)-1H-pyrrole-2,5-diyl)bis(2-(3,4-dimethoxyphenyl)acetate):

Yellow solid with two isomers (1:0.28), IR $v_{\max } 2952,1748,1252 \mathrm{~cm}^{-1}$; Isomer A: ${ }^{1} \mathrm{H}$ NMR $\left(\mathrm{CDCl}_{3}, 400 \mathrm{MHz}\right) \delta_{\mathrm{H}} 7.35(\mathrm{~s}, 2 \mathrm{H}), 7.04(\mathrm{~d}, J=8.5 \mathrm{~Hz}, 2 \mathrm{H}), 6.76(\mathrm{~d}, J=8.5 \mathrm{~Hz}$, 2H), $6.72(\mathrm{~d}, J=8.4 \mathrm{~Hz}, 2 \mathrm{H}), 6.59$ (d, $J=8.4 \mathrm{~Hz}, 2 \mathrm{H}), 6.52(\mathrm{~s}, 2 \mathrm{H}), 5.41$ (s, 2H), 4.90-4.86 (m, 3H), $4.74(\mathrm{~d}, J=11.8 \mathrm{~Hz}, 2 \mathrm{H}), 4.43(\mathrm{~d}, J=18.7 \mathrm{~Hz}, 1 \mathrm{H}), 3.84(\mathrm{~s}, 6 \mathrm{H})$, $3.82(\mathrm{~s}, 6 \mathrm{H}), 3.76(\mathrm{~s}, 6 \mathrm{H}), 3.29(\mathrm{~s}, 3 \mathrm{H}) ;{ }^{13} \mathrm{C} \mathrm{NMR}\left(\mathrm{CDCl}_{3}, 100 \mathrm{MHz}\right) \delta_{\mathrm{C}} 170.3,168.7$, $153.8,148.9,148.3,132.3,130.5,128.0,127.5,126.3,124.7,122.0,120.1,111.8$, 111.7, 111.0, 94.8, 74.7, 56.1, 56.0, 55.8, 51.8, 47.9, 46.8. Isomer B: ${ }^{1} \mathrm{H} \mathrm{NMR}\left(\mathrm{CDCl}_{3}\right.$, 
$400 \mathrm{MHz}) \delta_{\mathrm{H}} 7.27(\mathrm{~s}, 2 \mathrm{H}), 6.98(\mathrm{~d}, J=8.4 \mathrm{~Hz}, 2 \mathrm{H}), 6.75-6.71(\mathrm{~m}, 4 \mathrm{H}), 6.66-6.63(\mathrm{~m}$, 4H), 5.34 (s, 2H), 4.86 (d, $J=11.9 \mathrm{~Hz}, 2 \mathrm{H}), 4.62$ (d, $J=11.9 \mathrm{~Hz}, 2 \mathrm{H}), 4.48$ (s, 2H), $3.84(\mathrm{~s}, 6 \mathrm{H}), 3.83(\mathrm{~d}, 6 \mathrm{H}), 3.76(\mathrm{~s}, 6 \mathrm{H}), 3.59(\mathrm{~s}, 3 \mathrm{H}) ;{ }^{13} \mathrm{C} \mathrm{NMR}\left(\mathrm{CDCl}_{3}, 100 \mathrm{MHz}\right) \delta_{\mathrm{C}}$ $170.0,169.5,153.8,149.1,148.5,132.3,130.4,128.0,127.8,126.1,125.2,121.9$, 120.0, 111.8, 111.6, 111.1, 94.8, 74.8, 56.2, 56.0, 55.9, 52.4, 47.8, 46.5. HRESIMS $m / z 1072.0006[\mathrm{M}+\mathrm{H}]^{+}$(calcd for $\mathrm{C}_{45} \mathrm{H}_{42} \mathrm{Cl}_{8} \mathrm{NO}_{12}$ 1072.0078).

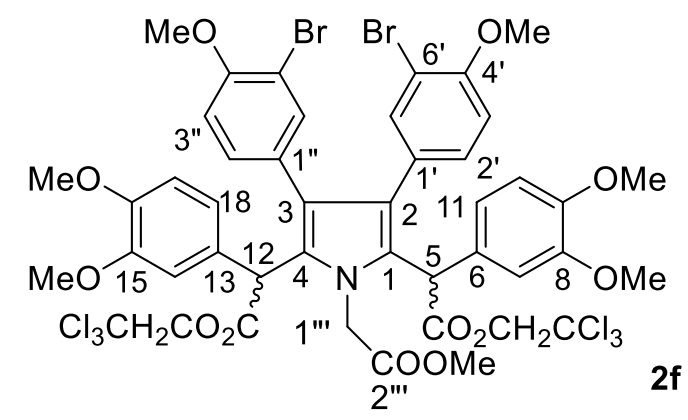

2f Bis(2,2,2-trichloroethyl)2,2'-(3,4-bis(3-bromo-4-methoxyphenyl)-1-(2methoxy-2-oxoethyl)-1H-pyrrole-2,5-diyl)bis(2-(3,4-dimethoxyphenyl)acetate):

Yellow solid, IR $v_{\max } 2917,1748,1250 \mathrm{~cm}^{-1} ;{ }^{1} \mathrm{H} \mathrm{NMR}\left(\mathrm{CDCl}_{3}, 400 \mathrm{MHz}\right) \delta_{\mathrm{H}} 7.51(\mathrm{~s}$, 2H), 7.07 (dd, $J=8.4,1.4 \mathrm{~Hz}, 2 \mathrm{H}), 6.74-6.71(\mathrm{~m}, 4 \mathrm{H}), 6.58(\mathrm{~d}, J=8.4 \mathrm{~Hz}, 2 \mathrm{H}), 6.52$ (d, $J=1.8 \mathrm{~Hz}, 2 \mathrm{H}), 5.41(\mathrm{~s}, 2 \mathrm{H}), 4.90-4.85(\mathrm{~m}, 3 \mathrm{H}), 4.73$ (d, $J=11.9 \mathrm{~Hz}, 2 \mathrm{H}), 4.43$ (d, $J=18.7 \mathrm{~Hz}, 1 \mathrm{H}), 3.823$ (s, 6H), 3.819 (s, 6H), 3.75 (s, 6H), $3.29(\mathrm{~s}, 3 \mathrm{H}) ;{ }^{13} \mathrm{C} \mathrm{NMR}$ $\left(\mathrm{CDCl}_{3}, 100 \mathrm{MHz}\right) \delta_{\mathrm{C}} 170.3,168.7,154.6,148.8,148.2,135.2,131.3,128.4,127.5$, 126.3, 124.6, 120.0, 111.6, 111.5, 111.2, 110.9, 94.8, 74.6, 56.2, 55.9, 55.8, 51.8, 47.9, 46.8; HRESIMS $m / z 1181.8993[\mathrm{M}+\mathrm{Na}]^{+}$(calcd for $\mathrm{C}_{45} \mathrm{H}_{41} \mathrm{Br}_{2} \mathrm{Cl}_{6} \mathrm{NO}_{12} \mathrm{Na} 1181.9077$ ).

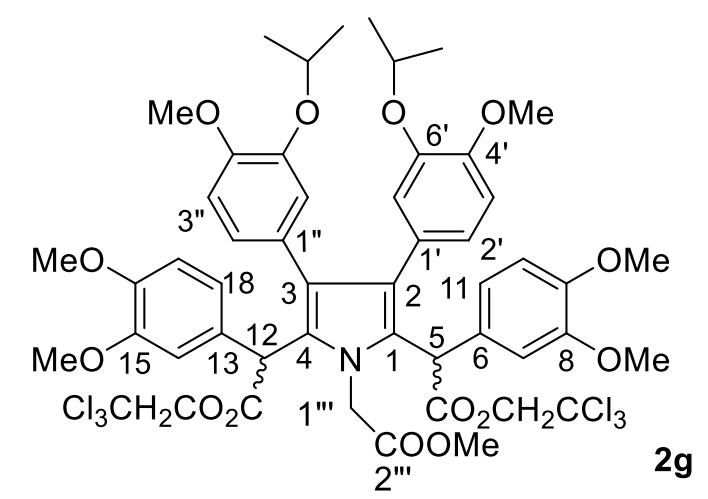

2g (Bis(2,2,2-trichloroethyl)2,2'-(3,4-bis(3-isopropoxy-4-methoxyphenyl)-1(2-methoxy-2-oxoethyl)-1H-pyrrole-2,5-diyl)bis(2-(3,4-dimethoxyphenyl)acetate):

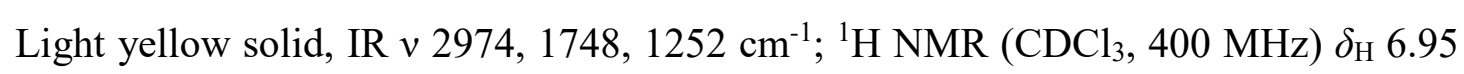


$(\mathrm{d}, J=7.8 \mathrm{~Hz}, 2 \mathrm{H}), 6.76-6.72(\mathrm{~m}, 4 \mathrm{H}), 6.65-6.61(\mathrm{~m}, 4 \mathrm{H}), 6.56(\mathrm{~s}, 2 \mathrm{H}), 5.56(\mathrm{~s}, 2 \mathrm{H})$, 4.87-4.80 (m, 3H), 4.69 (d, $J=11.9 \mathrm{~Hz}, 2 \mathrm{H}), 4.46$ (d, $J=18.8 \mathrm{~Hz}, 1 \mathrm{H}), 4.24-4.18$ (m, 2H), $3.83(\mathrm{~s}, 6 \mathrm{H}), 3.78(\mathrm{~s}, 6 \mathrm{H}), 3.76(\mathrm{~s}, 6 \mathrm{H}), 3.30(\mathrm{~s}, 3 \mathrm{H}), 1.18(\mathrm{~d}, J=6.0 \mathrm{~Hz}, 6 \mathrm{H})$, $1.06(\mathrm{~d}, J=5.9 \mathrm{~Hz}, 6 \mathrm{H}) ;{ }^{13} \mathrm{H}$ NMR $\left(\mathrm{CDCl}_{3}, 100 \mathrm{MHz}\right) \delta_{\mathrm{C}} 170.6,168.9,149.2,148.8$, $148.2,146.8,128.0,127.6,126.0,125.7,123.5,120.2,119.1,111.8,111.7,111.0,94.9$, 74.6, 71.2, 56.0, 56.0, 55.8, 51.8, 48.0, 46.9, 22.1, 21.7; HRESIMS $m / z 1117.6605$ $[\mathrm{M}+\mathrm{H}]^{+}$(calcd for $\mathrm{C}_{51} \mathrm{H}_{56} \mathrm{Cl}_{6} \mathrm{NO}_{14}$ 1118.1724).

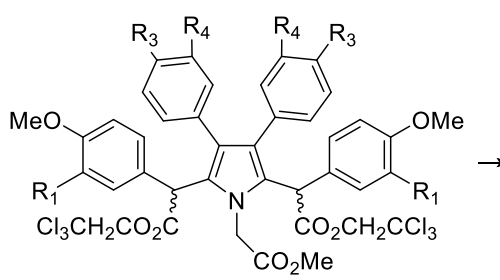

2a. $\mathrm{R}_{1}=\mathrm{R}_{3}=\mathrm{R}_{4}=\mathrm{OMe}$

2b. $R_{1}=O i P r, R_{3}=R_{4}=O M e$

2c. $R_{1}=O M e, R_{3}=R_{4}=H$

2d. $R_{1}=R_{3}=O M e, R_{4}=H$

2e. $R_{1}=R_{3}=O M e, R_{4}=C l$

2f. $R_{1}=R_{3}=O M e, R_{4}=B r$

2g. $R_{1}=R_{3}=O M e, R_{4}=O i P r$

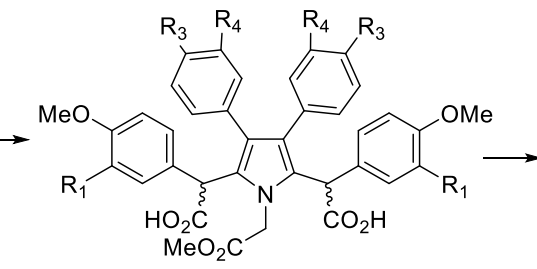

3a. $R_{1}=R_{3}=R_{4}=O M e$

3b. $R_{1}=O i P r, R_{3}=R_{4}=O M e$

3c. $\mathrm{R}_{1}=\mathrm{OMe}, \mathrm{R}_{3}=\mathrm{R}_{4}=\mathrm{H}$

3d. $R_{1}=R_{3}=O M e, R_{4}=H$

3e. $R_{1}=R_{3}=O M e, R_{4}=C l$

3f. $R_{1}=R_{3}=O M e, R_{4}=B r$

3g. $\mathrm{R}_{1}=\mathrm{R}_{3}=\mathrm{OMe}, \mathrm{R}_{4}=\mathrm{OiPr}$

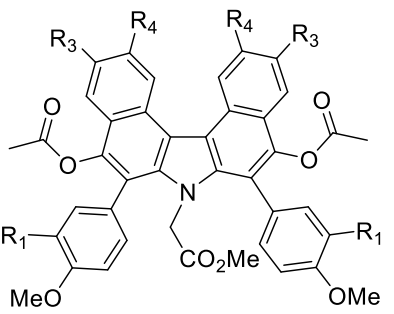

4a. $\mathrm{R}_{1}=\mathrm{R}_{3}=\mathrm{R}_{4}=\mathrm{OMe}$

4b. $R_{1}=O i P r, R_{3}=R_{4}=O M e$

4c. $R_{1}=O M e, R_{3}=R_{4}=H$

4d. $\mathrm{R}_{1}=\mathrm{R}_{3}=\mathrm{OMe}, \mathrm{R}_{4}=\mathrm{H}$

4e. $R_{1}=R_{3}=O M e, R_{4}=C l$

4f. $R_{1}=R_{3}=O M e, R_{4}=B r$

4g. $R_{1}=R_{3}=O M e, R_{4}=O i P r$

\section{Scheme S6. Synthesis of 4 a to $4 \mathrm{~g}$}

Synthesis of 4 a to $4 \mathrm{~g}$. A $1 \mathrm{~N}$ aqueous solution of ammonium acetate (4.0 eq.) and zinc power (50 eq.) were added to a solution of $2 \mathbf{2}$ (1.0 eq.) in tetrahydrofuran. The mixture was stirred at $\mathrm{rt}$ for $1 \mathrm{~h}$. The solid was then washed with tetrahydrofuran (20 $\mathrm{mL}$ ). The filtrate was concentrated under vacuum resulting in the crude 3a. The crude 3a was retaken into acetic anhydride, and then KOAc (0.26 eq.) was added. The mixture was refluxed for $2 \mathrm{hrs}$. After cooling to rt, the $\mathrm{pH}$ of this aqueous mixture was adjusted to 7-8 with saturated $\mathrm{NaHCO}_{3}$ solution. This mixture was extracted with EtOAc, while the organic layer was concentrated under vacuo. The crude extract was purified by silica chromatography eluding with $\mathrm{CH}_{2} \mathrm{Cl}_{2}-\mathrm{MeOH}$ (5:1) to yield 4a. Compounds $\mathbf{4 b}$ ( $72 \%$ yield), $\mathbf{4 c}$ (58\% yield), $\mathbf{4 d}$ (20\% yield), $\mathbf{4 e}$ (34\% yield), $\mathbf{4 f}$ (35\% yield), and $\mathbf{4 g}$ (39\% yield) were derived from $\mathbf{2 c - 2 g}$ respectively by the same protocol as for $\mathbf{4 a}$. 


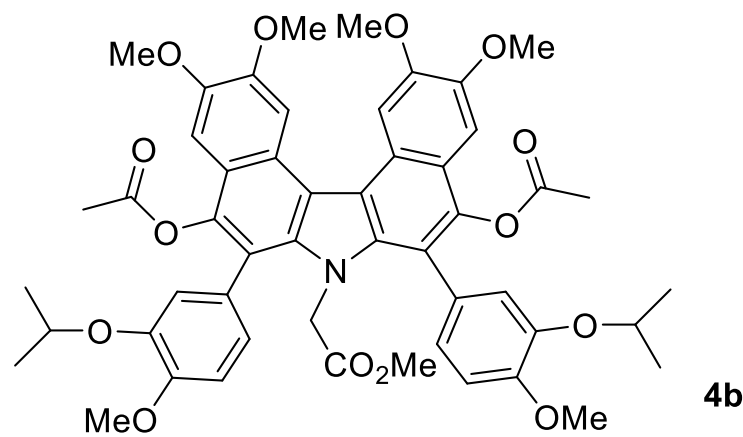

4b

(6,8-Bis(3-isopropoxy-4-methoxyphenyl)-2,3,11,12-tetramethoxy-7-(2-

methoxy-2-oxoethyl)-7H-dibenzo[c,g]carbazole-5,9-diyldiacetate)): Brown solid, IR $v_{\max } 2924,1748,1251 \mathrm{~cm}^{-1} ;{ }^{1} \mathrm{H} \mathrm{NMR}\left(\mathrm{CDCl}_{3}, 400 \mathrm{MHz}\right) \delta_{\mathrm{H}} 8.26(\mathrm{~s}, 2 \mathrm{H}), 7.19(\mathrm{~s}$, 2H), 6.96-6.79 (m, 6H), 4.45-4.41 (m, 2H), $4.36(\mathrm{~d}, J=19.0 \mathrm{~Hz}, 1 \mathrm{H}), 4.20(\mathrm{~d}, J=$ $19.0 \mathrm{~Hz}, 1 \mathrm{H}), 4.04(\mathrm{~s}, 6 \mathrm{H}), 3.97(\mathrm{~s}, 6 \mathrm{H}), 3.89(\mathrm{~s}, 6 \mathrm{H}), 3.34(\mathrm{~s}, 3 \mathrm{H}), 2.02(\mathrm{~s}, 6 \mathrm{H})$, 1.35-1.33 (m, 12H); ${ }^{13} \mathrm{C} \mathrm{NMR}\left(\mathrm{CDCl}_{3}, 100 \mathrm{MHz}\right) \delta_{\mathrm{C}} 169.7,169.4,148.9,148.2$, $146.8,142.9,136.0,126.5,123.5,123.0,118.3,118.2,117.4,116.1,116.0,111.4$, 106.3, 101.7, 71.1, 56.2, 56.0, 55.8, 51.4, 47.5, 21.0, 20.4; HRESIMS m/z 926.3314 $[\mathrm{M}+\mathrm{Na}]^{+}$(calcd for $\left.\mathrm{C}_{51} \mathrm{H}_{53} \mathrm{NO}_{14} \mathrm{Na} 926.3466\right)$

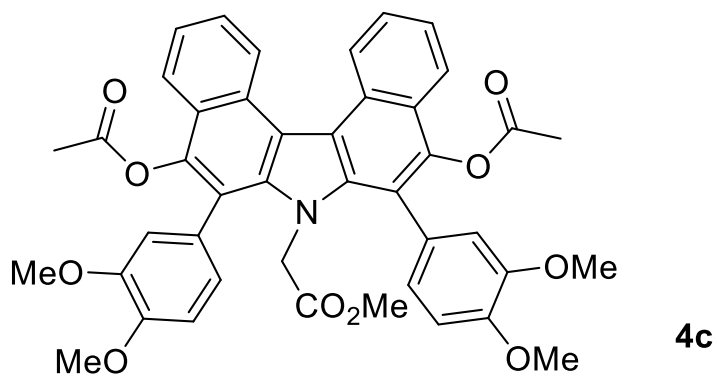

4c (6,8-Bis(3,4-dimethoxyphenyl)-7-(2-methoxy-2-oxoethyl)-7H-dibenzo[c,g] carbazole-5,9-diyl diacetate): Brown solid, ${ }^{1} \mathrm{H} \mathrm{NMR}\left(\mathrm{CDCl}_{3}, 400 \mathrm{MHz}\right) \delta_{\mathrm{H}} 9.14(\mathrm{~d}$, $J=8.4 \mathrm{~Hz}, 2 \mathrm{H}), 7.91(\mathrm{~d}, J=8.4 \mathrm{~Hz}, 2 \mathrm{H}), 7.69-7.65(\mathrm{~m}, 2 \mathrm{H}), 7.58-7.54(\mathrm{~m}, 2 \mathrm{H}), 6.91$ (s, 2H), 6.86-6.80 (m, 4H), 4.45-4.23 (m, 2H), $3.92(\mathrm{~s}, 6 \mathrm{H}), 3.81(\mathrm{~s}, 6 \mathrm{H}), 3.35(\mathrm{~s}, 3 \mathrm{H})$, $2.06(\mathrm{~s}, 6 \mathrm{H}) ;{ }^{13} \mathrm{C} \mathrm{NMR}\left(\mathrm{CDCl}_{3}, 100 \mathrm{MHz}\right) \delta_{\mathrm{C}} 170.0,169.6,149.0,148.4,144.1,136.2$, $128.3,126.7,125.9,125.5,124.7,123.3,122.7,122.4,119.4,117.1,114.1,110.9,56.1$, 56.0, 51.4, 47.9, 20.5. HRESIMS $m / z$ 728.2491 $[\mathrm{M}+\mathrm{H}]^{+}\left(\right.$calcd for $\mathrm{C}_{43} \mathrm{H}_{38} \mathrm{NO}_{10}$ 728.2495). 


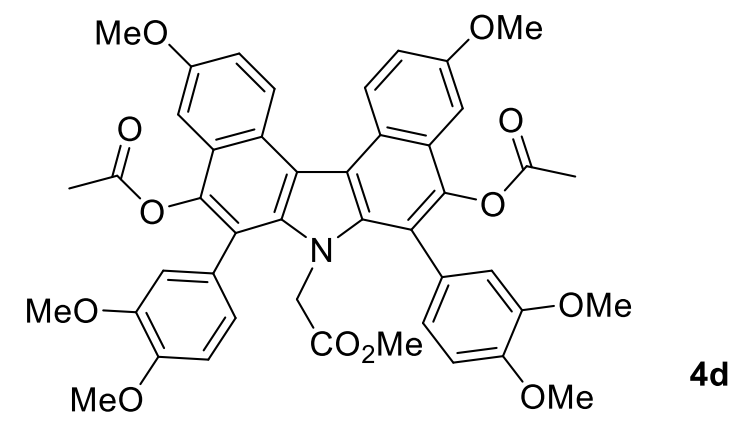

4d (6,8-Bis(3,4-dimethoxyphenyl)-3,11-dimethoxy-7-(2-methoxy-2-oxoethyl) -7H-dibenzo[c,g]carbazole-5,9-diyl diacetate): Brown solid, IR $v_{\max }$ 2935, 1750, $1257 \mathrm{~cm}^{-1} ;{ }^{1} \mathrm{H} \mathrm{NMR}\left(\mathrm{CDCl}_{3}, 400 \mathrm{MHz}\right) \delta_{\mathrm{H}} 9.02(\mathrm{~d}, J=9.2 \mathrm{~Hz}, 2 \mathrm{H}), 7.34(\mathrm{dd}, J=9.2$, $2.7 \mathrm{~Hz}, 2 \mathrm{H}), 7.21(\mathrm{~d}, J=2.7 \mathrm{~Hz}, 2 \mathrm{H}), 6.90-6.80(\mathrm{~m}, 6 \mathrm{H}), 4.37$ (d, $J=19.1 \mathrm{~Hz}, 1 \mathrm{H})$, $4.22(\mathrm{~d}, J=19.1 \mathrm{~Hz}, 1 \mathrm{H}), 3.97(\mathrm{~s}, 6 \mathrm{H}), 3.92(\mathrm{~s}, 6 \mathrm{H}), 3.81(\mathrm{~s}, 6 \mathrm{H}), 3.34(\mathrm{~s}, 3 \mathrm{H}), 2.05(\mathrm{~s}$, $6 \mathrm{H}) ;{ }^{13} \mathrm{C} \mathrm{NMR}\left(\mathrm{CDCl}_{3}, 100 \mathrm{MHz}\right) \delta_{\mathrm{C}} 169.8,169.7,156.7,148.9,148.7,143.2,143.2$, 135.1, 127.0, 126.8, 124.5, 123.4, 122.6, 119.8, 117.0, 116.9, 110.8, 102.1, 56.0, 56.0, 55.5, 51.3, 47.7, 20.5; HRESIMS $m / z$ 810.2508 $[\mathrm{M}+\mathrm{Na}]^{+}$(calcd for $\mathrm{C}_{45} \mathrm{H}_{41} \mathrm{NO}_{12} \mathrm{Na}$ 810.2629).

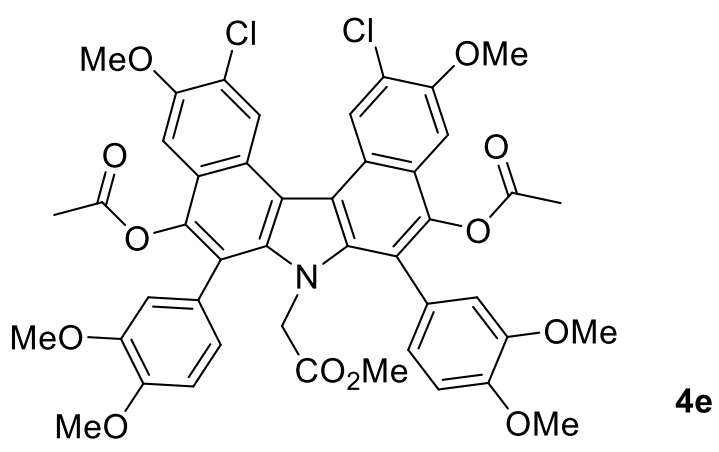

4e (2,12-Dichloro-6,8-bis(3,4-dimethoxyphenyl)-3,11-dimethoxy-7-(2-methoxy -2-oxoethyl)-7H-dibenzo[c,g]carbazole-5,9-diyl diacetate): Brown solid, IR $v_{\max }$ 2951, 1748, $1239 \mathrm{~cm}^{-1} ;{ }^{1} \mathrm{H}$ NMR $\left(\mathrm{CDCl}_{3}, 400 \mathrm{MHz}\right) \delta_{\mathrm{H}} 9.13(\mathrm{~s}, 2 \mathrm{H}), 7.22(\mathrm{~s}, 2 \mathrm{H})$, 6.91-6.76 (m, 6H), $4.36(\mathrm{~d}, J=19.0 \mathrm{~Hz}, 1 \mathrm{H}), 4.21(\mathrm{~d}, J=19.0 \mathrm{~Hz}, 1 \mathrm{H}), 4.04(\mathrm{~s}, 6 \mathrm{H})$, $3.92(\mathrm{~s}, 6 \mathrm{H}), 3.80(\mathrm{~s}, 6 \mathrm{H}), 3.35(\mathrm{~s}, 3 \mathrm{H}), 2.04(\mathrm{~s}, 6 \mathrm{H}) ;{ }^{13} \mathrm{C} \mathrm{NMR}\left(\mathrm{CDCl}_{3}, 100 \mathrm{MHz}\right) \delta_{\mathrm{C}}$ $169.6,169.5,152.4,149.0,143.2,143.2,135.8,126.6,126.4,124.0,123.2,123.2$, 122.6, 120.0, 115.9, 110.9, 102.5, 99.7, 56.3, 56.1, 56.0, 51.4, 47.7, 20.5; HRESIMS $m / z 878.1739[\mathrm{M}+\mathrm{Na}]^{+}$(calcd for $\mathrm{C}_{45} \mathrm{H}_{39} \mathrm{Cl}_{2} \mathrm{NO}_{12} \mathrm{Na} 878.1849$ ). 


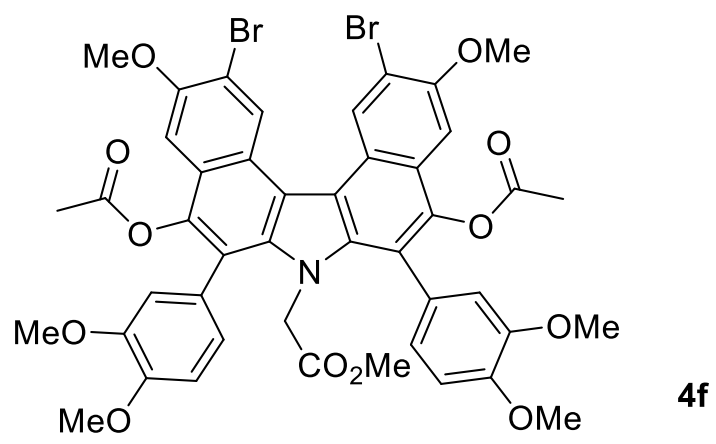

4f (2,12-Dibromo-6,8-bis(3,4-dimethoxyphenyl)-3,11-dimethoxy-7-(2-methoxy -2-oxoethyl)-7H-dibenzo[c,g]carbazole-5,9-diyl diacetate): Yellow solid, IR $v_{\max }$ 2951, 1770, $1258 \mathrm{~cm}^{-1} ;{ }^{1} \mathrm{H} \mathrm{NMR}\left(\mathrm{CDCl}_{3}, 400 \mathrm{MHz}\right) \delta_{\mathrm{H}} 9.33(\mathrm{~s}, 2 \mathrm{H}), 7.19(\mathrm{~s}, 2 \mathrm{H})$, 6.89-6.76 (m, 6H), $4.36(\mathrm{~d}, J=19.0 \mathrm{~Hz}, 1 \mathrm{H}), 4.20(\mathrm{~d}, J=19.0 \mathrm{~Hz}, 1 \mathrm{H}), 4.04(\mathrm{~s}, 6 \mathrm{H})$, $3.92(\mathrm{~s}, 6 \mathrm{H}), 3.80(\mathrm{~s}, 6 \mathrm{H}), 3.34(\mathrm{~s}, 3 \mathrm{H}), 2.04(\mathrm{~s}, 6 \mathrm{H}) ;{ }^{13} \mathrm{C} \mathrm{NMR}\left(\mathrm{CDCl}_{3}, 100 \mathrm{MHz}\right) \delta_{\mathrm{C}}$ $169.7,169.5,153.1,149.0,148.9,148.3,143.2,135.7,130.1,126.4,123.7,123.7$, 122.5, 120.2, 115.8, 113.3, 110.8, 102.1, 56.4, 56.0, 56.0, 51.4, 47.7, 20.5; HRESIMS $m / z 966.1042[\mathrm{M}+\mathrm{Na}]^{+}$(calcd for $\mathrm{C}_{45} \mathrm{H}_{39} \mathrm{Br}_{2} \mathrm{NO}_{12} \mathrm{Na} 966.0839$ ).

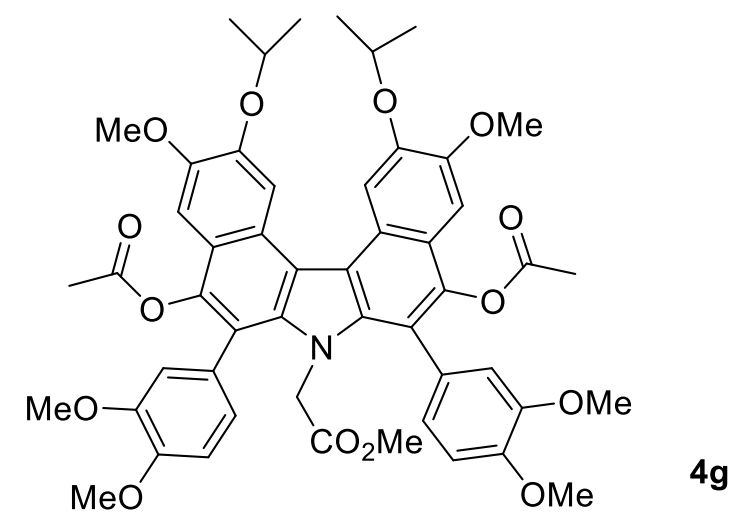

$4 \mathrm{~g}$ (6,8-bis(3,4-dimethoxyphenyl)-2,12-diisopropoxy-3,11-dimethoxy-7-(2methoxy-2-oxoethyl)-7H-dibenzo[c,g]carbazole-5,9-diyl diacetate): Yellow solid, IR v 2933, 1756, $1240 \mathrm{~cm}^{-1} ;{ }^{1} \mathrm{H}$ NMR $\left(\mathrm{CDCl}_{3}, 400 \mathrm{MHz}\right) \delta 8.44(\mathrm{~s}, 2 \mathrm{H}), 7.19(\mathrm{~s}, 2 \mathrm{H})$, 6.89-6.79 (m, 4H), 4.79-4.73 (m, 2H), $4.36(\mathrm{~d}, J=19.0 \mathrm{~Hz}, 1 \mathrm{H}), 4.21(\mathrm{~d}, J=19.0 \mathrm{~Hz}$, 1H), $4.01(\mathrm{~s}, 6 \mathrm{H}), 3.91(\mathrm{~s}, 6 \mathrm{H}), 3.80(\mathrm{~s}, 6 \mathrm{H}), 3.34(\mathrm{~s}, 3 \mathrm{H}), 2.03(\mathrm{~s}, 6 \mathrm{H}), 1.37(\mathrm{~s}, 12 \mathrm{H})$; ${ }^{13} \mathrm{C} \mathrm{NMR}\left(\mathrm{CDCl}_{3}, 100 \mathrm{MHz}\right) \delta 169.8,169.6,149.5,148.7,147.4,143.0,135.6,129.4$, $126.9,123.8,122.8,118.5,117.3,116.1,115.3,110.7,110.6,102.3,72.0,55.9,55.9$, 55.9, 51.2, 47.6, 22.2, 20.4; HRESIMS $m / z$ 904.3673 [M+H] ${ }^{+}\left(\right.$calcd for $\mathrm{C}_{51} \mathrm{H}_{54} \mathrm{NO}_{14}$ 904.3466). 


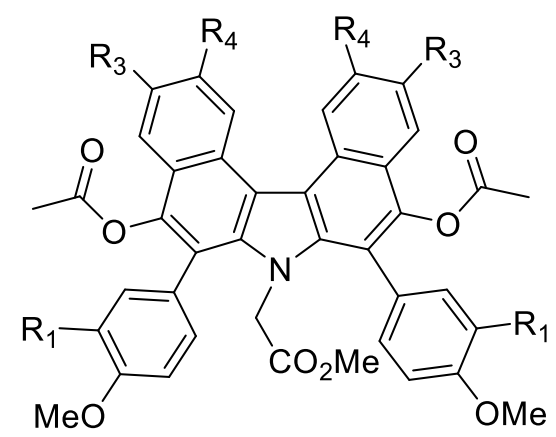

4a. $\mathrm{R}_{1}=\mathrm{R}_{3}=\mathrm{R}_{4}=\mathrm{OMe}$

4b. $\mathrm{R}_{1}=\mathrm{OiPr}, \mathrm{R}_{3}=\mathrm{R}_{4}=\mathrm{OMe}$

4c. $\mathrm{R}_{1}=\mathrm{OMe}, \mathrm{R}_{3}=\mathrm{R}_{4}=\mathrm{H}$

4d. $R_{1}=R_{3}=O M e, R_{4}=H$

4e. $R_{1}=R_{3}=O M e, R_{4}=\mathrm{Cl}$

4f. $R_{1}=R_{3}=O M e, R_{4}=B r$

4g. $R_{1}=R_{3}=O M e, R_{4}=O i P r$

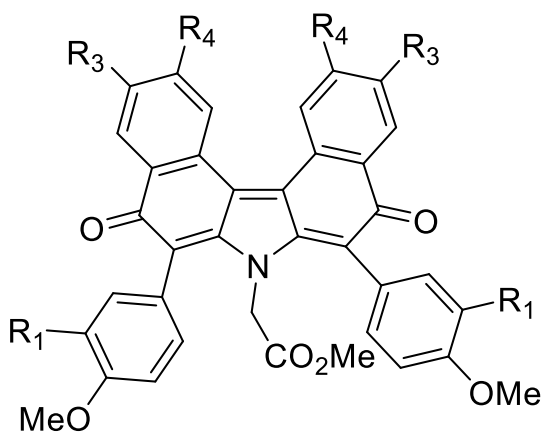

II-135. $\mathrm{R}_{1}=\mathrm{R}_{3}=\mathrm{R}_{4}=\mathrm{OMe}$

5. $R_{1}=O i P r, R_{3}=R_{4}=O M e$

13. $R_{1}=O M e, R_{3}=R_{4}=H$

14. $R_{1}=R_{3}=O M e, R_{4}=H$

15. $R_{1}=R_{3}=O M e, R_{4}=C l$

16. $R_{1}=R_{3}=O M e, R_{4}=B r$

17. $R_{1}=R_{3}=O M e, R_{4}=O i P r$

\section{Scheme S7. Synthesis of II-135, 5 and 13 to 17}

Synthesis of II-135, 5 and 13 to 17. An aqueous solution of $\mathrm{NaHCO}_{3}(5 \%$, some drops) was added to $4 \mathrm{a}$ (1.0 eq.) in $\mathrm{MeOH}$. The mixture was stirred at $55{ }^{\circ} \mathrm{C}$ for $1 \mathrm{~h}$ in air. After cooling to $\mathrm{rt}, \mathrm{HCl}$ solution $(6 \%)$ and $\mathrm{H}_{2} \mathrm{O}$ were added subsequently under stirring. The mixture was extracted with EtOAc to get an organic layer which was washed with brine and then concentrated under vacuo. The crude extract was purified by silica chromatography eluting with $\mathrm{CH}_{2} \mathrm{Cl}_{2}-\mathrm{MeOH}$ (5:1) to yield $\mathbf{I I - 1 3 5}$ (yield 81\%). Analogues 5 (yield 90\%), 13 (yield 67\%), 14 (yield 51\%), 15 (yield 13\%), 16 (yield 41\%) and 17 (yield: 89\%) were derived from $\mathbf{4 b}$ to $\mathbf{4 g}$ respectively by the same protocol as for II-135.<smiles></smiles>

II-135 (Methyl-2-(6,8-bis(3,4-dimethoxyphenyl)-2,3,11,12-tetramethoxy-5,9-

dioxo-5H-dibenzo[c,g] carbazol-7(9H)-yl)acetate): Brown solid, IR $v_{\max }$ 2951, 1751, $1738,1271 \mathrm{~cm}^{-1} ;{ }^{1} \mathrm{H}$ NMR $\left(\mathrm{CDCl}_{3}, 400 \mathrm{MHz}\right) \delta_{\mathrm{H}} 7.92(\mathrm{~s}, 2 \mathrm{H}), 7.66(\mathrm{~s}, 2 \mathrm{H}), 6.86(\mathrm{~d}, J$ $=8.2 \mathrm{~Hz}, 2 \mathrm{H}), 6.77-6.74(\mathrm{~m}, 4 \mathrm{H}), 4.00(\mathrm{~s}, 6 \mathrm{H}), 3.89(\mathrm{~s}, 6 \mathrm{H}), 3.87(\mathrm{~s}, 6 \mathrm{H}), 3.84(\mathrm{~s}, 6 \mathrm{H})$, 
3.72-3.69 (m, 2H), $3.38(\mathrm{~s}, 3 \mathrm{H}) ;{ }^{13} \mathrm{C} \mathrm{NMR}\left(\mathrm{CDCl}_{3}, 100 \mathrm{MHz}\right) \delta_{\mathrm{C}} 183.6,169.0,153.4$, $151.3,151.3,149.2,148.9,130.6,126.2,125.0$, 124.2, 123.4, 117.3, 114.1, 111.1, $110.0,109.0,56.7,56.4,56.0,56.0,51.8,47.3$; HRESIMS $m / z 762.2568[\mathrm{M}+\mathrm{H}]^{+}$ (calcd for $\mathrm{C}_{43} \mathrm{H}_{40} \mathrm{NO}_{12} 762.2472$ ).

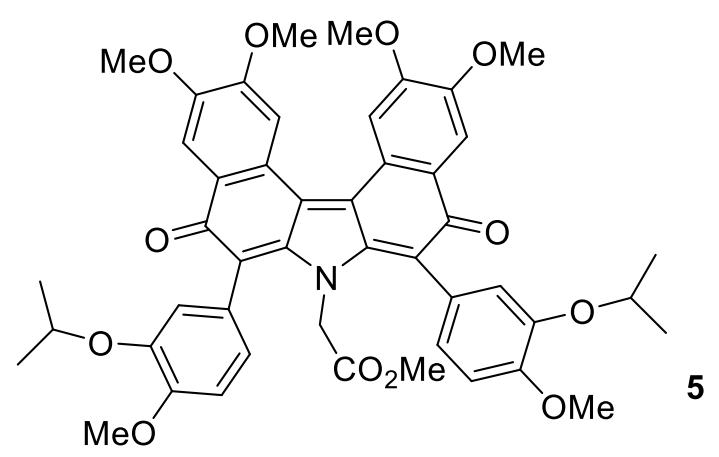

5 (Methyl-2-(6,8-bis(3-isopropoxy-4-methoxyphenyl)-2,3,11,12-tetramethoxy -5,9-dioxo-5H-dibenzo [c,g]carbazol-7(9H)-yl)acetate): Brown solid, IR v 2973, 1748, $1263 \mathrm{~cm}^{-1} ;{ }^{1} \mathrm{H}$ NMR $\left(\mathrm{CDCl}_{3}, 400 \mathrm{MHz}\right) \delta 7.92(\mathrm{~s}, 2 \mathrm{H}), 7.66(\mathrm{~s}, 2 \mathrm{H}), 6.85(\mathrm{~d}, J=$ 8.1 Hz, 2H), 6.77-6.74 (m, 4H), 4.51-4.45 (m, 2H), $4.00(\mathrm{~s}, 6 \mathrm{H}), 3.864(\mathrm{~s}, 6 \mathrm{H}), 3.856$ $(\mathrm{s}, 6 \mathrm{H}), 3.76-3.67(\mathrm{~m}, 2 \mathrm{H}), 3.37(\mathrm{~s}, 3 \mathrm{H}), 1.35(\mathrm{~d}, J=5.9 \mathrm{~Hz}, 12 \mathrm{H}) ;{ }^{13} \mathrm{C} \mathrm{NMR}\left(\mathrm{CDCl}_{3}\right.$, $100 \mathrm{MHz}) \delta 183.5,168.8,151.2,151.2,150.5,147.2,130.7,126.2,124.8,124.2$, $118.2,118.2,117.3,111.6,109.9,108.9,71.5,56.6,56.3,56.0,51.9,47.3,22.2$; HRESIMS $m / z$ 818.3181 $[\mathrm{M}+\mathrm{H}]^{+}$(calcd for $\mathrm{C}_{47} \mathrm{H}_{48} \mathrm{NO}_{12}$ 818.3098).

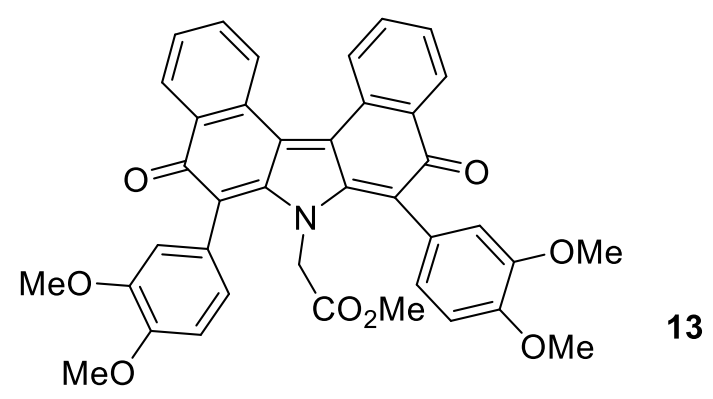

13

(Methyl-2-(6,8-bis(3,4-dimethoxyphenyl)-5,9-dioxo-5H-dibenzo[c,g] carbazol-7(9H)-yl)acetate): Brown solid, ${ }^{1} \mathrm{H} \mathrm{NMR}\left(\mathrm{CDCl}_{3}, 400 \mathrm{MHz}\right) \delta_{\mathrm{H}} 8.52-8.50$ (m, 2H), 8.23-8.21 (m, 2H), 7.58-7.55 (m, 4H), 6.88-6.86 (m, 2H), 6.79-6.75 (m, 4H), $3.89(\mathrm{~s}, 6 \mathrm{H}), 3.84(\mathrm{~s}, 6 \mathrm{H}), 3.79-3.78(\mathrm{~m}, 2 \mathrm{H}), 3.38(\mathrm{~s}, 3 \mathrm{H}) ;{ }^{13} \mathrm{C} \mathrm{NMR}\left(\mathrm{CDCl}_{3}, 100\right.$ $\mathrm{MHz}) \delta_{\mathrm{C}} 184.1,168.9,168.8,149.3,148.9,131.6,131.3,131.2,130.8,130.1,128.3$, $126,5,124.8,123.4,118.0,114.0,111.1,56.1,56.0,51.9,47.4$; HRESIMS $m / z$ 
$642.2133[\mathrm{M}+\mathrm{H}]^{+}$(calcd for $\left.\mathrm{C}_{39} \mathrm{H}_{32} \mathrm{NO}_{8} 642.2050\right)$.

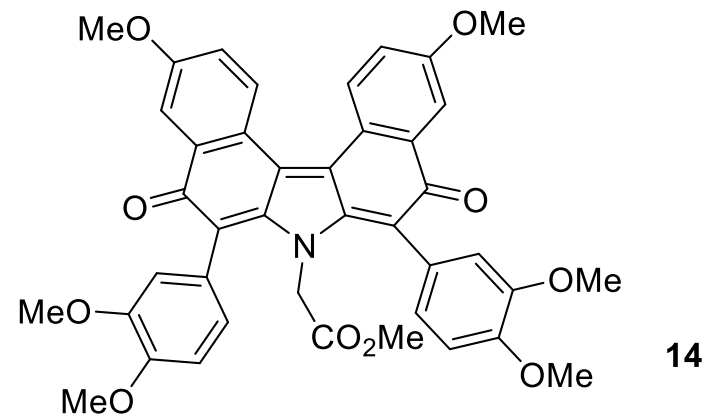

14 (Methyl-2-(6,8-bis(3,4-dimethoxyphenyl)-3,11-dimethoxy-5,9-dioxo-5Hdibenzo[c,g]carbazol-7(9H)-yl)acetate): Brown solid, IR $v_{\max }$ 2949, 1752, 1621, $1251 \mathrm{~cm}^{-1} ;{ }^{1} \mathrm{H}$ NMR $\left(\mathrm{CDCl}_{3}, 400 \mathrm{MHz}\right) \delta_{\mathrm{H}} 8.34(\mathrm{~d}, J=8.8 \mathrm{~Hz}, 2 \mathrm{H}), 7.67(\mathrm{~d}, J=2.9$ Hz, 2H), 7.07 (dd, $J=8.8,2.9 \mathrm{~Hz}, 2 \mathrm{H}), 6.85$ (d, $J=8.2 \mathrm{~Hz}, 2 \mathrm{H}), 6.77-6.74$ (m, 4H), $3.91(\mathrm{~s}, 6 \mathrm{H}), 3.87(\mathrm{~s}, 6 \mathrm{H}), 3.83(\mathrm{~s}, 6 \mathrm{H}), 3.77-3.69(\mathrm{~m}, 2 \mathrm{H}), 3.36(\mathrm{~s}, 3 \mathrm{H}) ;{ }^{13} \mathrm{C} \mathrm{NMR}$ $\left(\mathrm{CDCl}_{3}, 100 \mathrm{MHz}\right) \delta_{\mathrm{C}} 184.0,168.9,161.2,153.7,149.1,148.8,133.0,129.9,128.2$, $124.9,123.3,123.2,118.9,117.7,113.9,110.9,110.9,56.0,55.9,55.8,51.7,47.3$; HRESIMS $m / z 702.2323[\mathrm{M}+\mathrm{H}]^{+}$(calcd for $\mathrm{C}_{41} \mathrm{H}_{36} \mathrm{NO}_{10} 702.2261$ ).<smiles></smiles>

15 (Methyl-2-(2,12-dichloro-6,8-bis(3,4-dimethoxyphenyl)-3,11-dimethoxy-5,9 -dioxo-5H-dibenzo[c,g] carbazol-7(9H)-yl)acetate): Brown solid, IR $v_{\max } 2919$, 1748, 1620, $1252 \mathrm{~cm}^{-1} ;{ }^{1} \mathrm{H}$ NMR $\left(\mathrm{CDCl}_{3}, 400 \mathrm{MHz}\right) \delta_{\mathrm{H}} 8.43(\mathrm{~s}, 2 \mathrm{H}), 7.74(\mathrm{~s}, 2 \mathrm{H})$, $6.87(\mathrm{~d}, J=8.1 \mathrm{~Hz}, 2 \mathrm{H}), 6.77-6.73(\mathrm{~m}, 4 \mathrm{H}), 4.04(\mathrm{~s}, 6 \mathrm{H}), 3.89(\mathrm{~s}, 6 \mathrm{H}), 3.84(\mathrm{~s}, 6 \mathrm{H})$, 3.78-3.71 (m, 2H), $3.38(\mathrm{~s}, 3 \mathrm{H}) ;{ }^{13} \mathrm{C} \mathrm{NMR}\left(\mathrm{CDCl}_{3}, 100 \mathrm{MHz}\right) \delta_{\mathrm{C}} 188.6,156.9,150.0$, $149.4,148.9,131.4,129.6,128.2,126.8,124.5,123.4,123.3,118.0,114.2,113.9$, 111.1, 110.6, 56.7, 56.1, 56.0, 51.9, 47.5; HRESIMS $m / z 770.1539[\mathrm{M}+\mathrm{H}]^{+}$(calcd for $\mathrm{C}_{41} \mathrm{H}_{34} \mathrm{Cl}_{2} \mathrm{NO}_{10}$ 770.1482). 


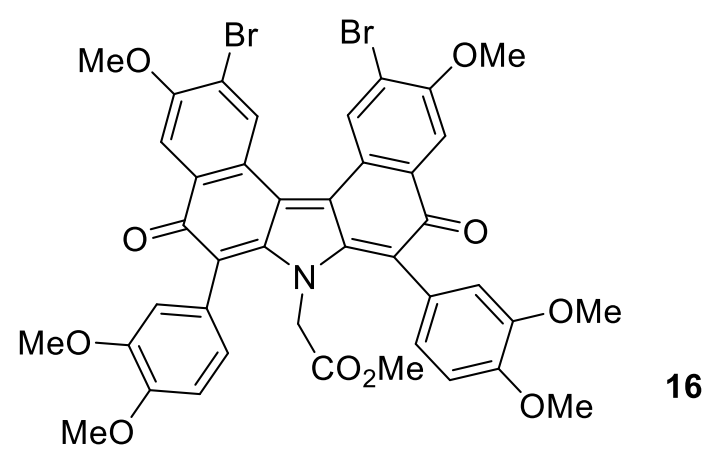

16 (Methyl-2-(2,12-dibromo-6,8-bis(3,4-dimethoxyphenyl)-3,11-dimethoxy -5,9-dioxo-5H-dibenzo[c,g] carbazol-7(9H)-yl)acetate): Brown solid, IR $v_{\max } 2920$, 1748, 1619, $1252 \mathrm{~cm}^{-1} ;{ }^{1} \mathrm{H} \mathrm{NMR}\left(\mathrm{CDCl}_{3}, 400 \mathrm{MHz}\right) \delta_{\mathrm{H}} 8.63(\mathrm{~s}, 2 \mathrm{H}), 7.69(\mathrm{~s}, 2 \mathrm{H})$, $6.87(\mathrm{~d}, J=8.2 \mathrm{~Hz}, 2 \mathrm{H}), 6.77-6.73(\mathrm{~m}, 4 \mathrm{H}), 4.04(\mathrm{~s}, 6 \mathrm{H}), 3.89(\mathrm{~s}, 6 \mathrm{H}), 3.84(\mathrm{~s}, 6 \mathrm{H})$, 3.75-3.70 (m, 2H), $3.38(\mathrm{~s}, 3 \mathrm{H}) ;{ }^{13} \mathrm{C} \mathrm{NMR}\left(\mathrm{CDCl}_{3}, 100 \mathrm{MHz}\right) \delta_{\mathrm{C}} 183.4,168.7,157.8$, $149.4,148.9,148.9,132.1,131.4,129.4,124.5,123.7,123.3,118.1,116.2,113.9$, 111.1, 110.3, 56.9, 56.0, 56.0, 51.9, 47.4; HRESIMS $m / z$ 857.8329 $[\mathrm{M}+\mathrm{H}]^{+}$(calcd for $\left.\mathrm{C}_{41} \mathrm{H}_{34} \mathrm{Br}_{2} \mathrm{NO}_{10} 858.0471\right)$.

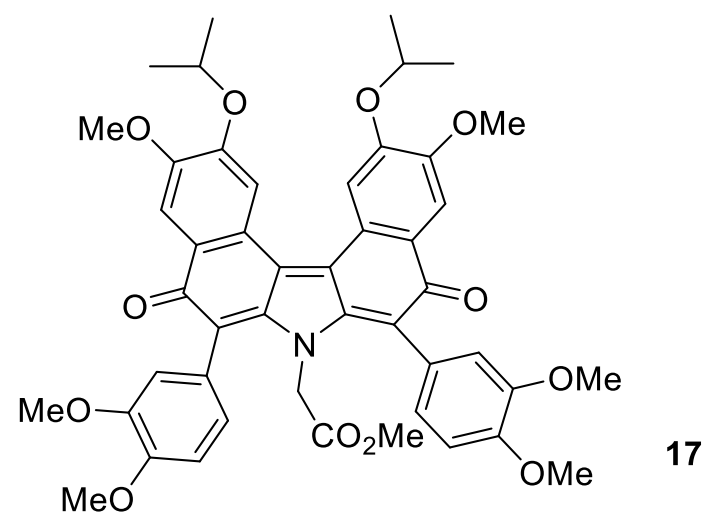

17

(Methyl-2-(6,8-bis(3,4-dimethoxyphenyl)-2,12-diisopropoxy-3,11dimethoxy-5,9-dioxo-5H-dibenzo [c,g]carbazol-7(9H)-yl)acetate): Brown solid, IR $v$ 2933, 1755, 1621, $1240 \mathrm{~cm}^{-1} ;{ }^{1} \mathrm{H}$ NMR $\left(\mathrm{CDCl}_{3}, 400 \mathrm{MHz}\right) \delta 7.90(\mathrm{~s}, 2 \mathrm{H}), 7.68(\mathrm{~s}$, 2H), $6.86(\mathrm{~d}, J=8.0 \mathrm{~Hz}, 2 \mathrm{H}), 6.77-6.74(\mathrm{~m}, 4 \mathrm{H}), 4.58-4.52(\mathrm{~m}, 2 \mathrm{H}), 3.98(\mathrm{~s}, 6 \mathrm{H})$, 3.89 (s, 6H), 3.83 (s, 6H), $3.72(\mathrm{bs}, 2 \mathrm{H}), 3.38(\mathrm{~s}, 3 \mathrm{H}), 1.35(\mathrm{~d}, J=5.9 \mathrm{~Hz}, 12 \mathrm{H}) ;{ }^{13} \mathrm{C}$ $\operatorname{NMR}\left(\mathrm{CDCl}_{3}, 100 \mathrm{MHz}\right) \delta 183.6,168.9,152.5,149.9,149.1,148.8,130.4,129.5$, $126.1,125.0,124.0,123.4,117.2,114.1,113.0,111.0,110.4,72.5,56.2,56.0,55.9$, 51.7, 47.3, 22.2; HRESIMS $m / z$ 818.3215 [M+H] $]^{+}$(calcd for $\mathrm{C}_{47} \mathrm{H}_{48} \mathrm{NO}_{12}$ 818.3098). 

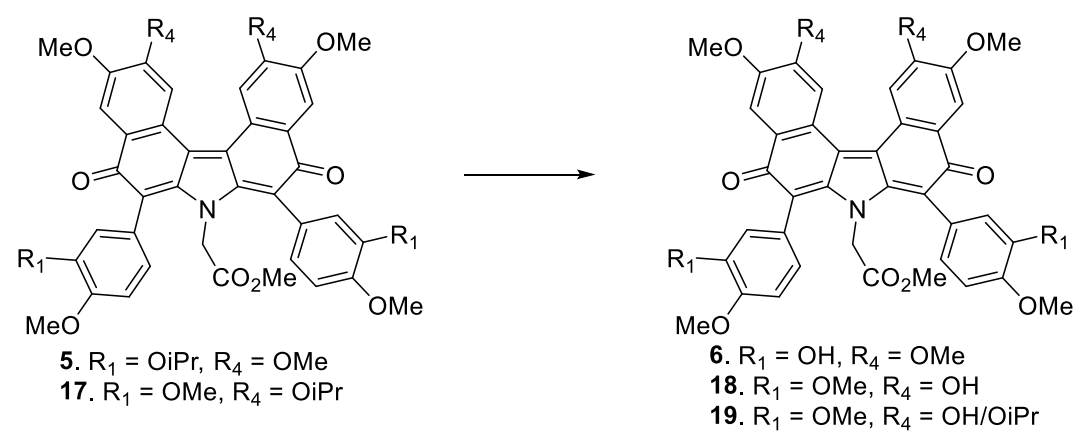

\section{Scheme S8. Synthesis of 6, 18 and 19}

Synthesis of 6, 18 and 19. Aluminium chloride $\left(\mathrm{AlCl}_{3}, 6.0\right.$ eq.) was added to 5 (1.0 eq.) in dry $\mathrm{CHCl}_{3}$ under $\mathrm{N}_{2}$. The mixture was stirred at $\mathrm{rt}$ for $18 \mathrm{~h}$. Saturated aqueous $\mathrm{NH}_{4} \mathrm{Cl}$ was added under stirring. The mixture was extracted with $\mathrm{CH}_{2} \mathrm{Cl}_{3}$, and the organic layer was washed with brine to concentrate under vacuo. The crude extract was purified by silica gel column eluting with $\mathrm{CH}_{2} \mathrm{Cl}_{2}-\mathrm{MeOH}(4: 1)$ to obtain 6 (yield 96\%). Conversion of 17 to 18 (yield 82\%) and 18 to 19 (yield 25\%) was performed by the same protocol as for $\mathbf{6}$.

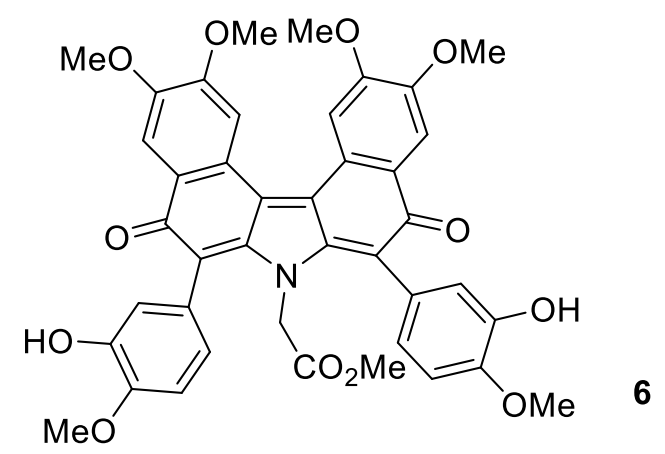

\section{6 (Methyl-2-(6,8-bis(3-hydroxy-4-methoxyphenyl)-2,3,11,12-tetramethoxy-}

5,9-dioxo-5H-dibenzo[c,g] carbazol-7(9H)-yl)acetate): Brown solid, IR $v_{\max } 2918$, $1748,1251 \mathrm{~cm}^{-1} ;{ }^{1} \mathrm{H}$ NMR $\left(\mathrm{CDCl}_{3}, 400 \mathrm{MHz}\right) \delta_{\mathrm{H}} 7.91(\mathrm{~s}, 2 \mathrm{H}), 7.65(\mathrm{~s}, 2 \mathrm{H}), 6.83(\mathrm{~d}, J$ $=8.2 \mathrm{~Hz}, 2 \mathrm{H}), 6.76(\mathrm{~s}, 2 \mathrm{H}), 6.68(\mathrm{~d}, J=8.2 \mathrm{~Hz}, 2 \mathrm{H}), 5.61(\mathrm{~s}, 2 \mathrm{H}), 4.00(\mathrm{~s}, 6 \mathrm{H}), 3.89$ $(\mathrm{s}, 6 \mathrm{H}), 3.87(\mathrm{~s}, 6 \mathrm{H}), 3.69(\mathrm{bs}, 2 \mathrm{H}), 3.48(\mathrm{~s}, 3 \mathrm{H}) ;{ }^{13} \mathrm{C} \mathrm{NMR}\left(\mathrm{CDCl}_{3}, 100 \mathrm{MHz}\right) \delta_{\mathrm{C}}$ $183.6,168.7,153.3,151.2,151.2,146.7,145.7,145.6,126.2,125.7,125.7,124.2$, 117.1, 117.1, 110.5, 109.9, 108.9, 56.6, 56.3, 56.0, 52.2, 47.4; HRESIMS $m / z$ 734.2295 $[\mathrm{M}+\mathrm{H}]^{+}$(calcd for $\mathrm{C}_{41} \mathrm{H}_{36} \mathrm{NO}_{12}$ 734.2159). 


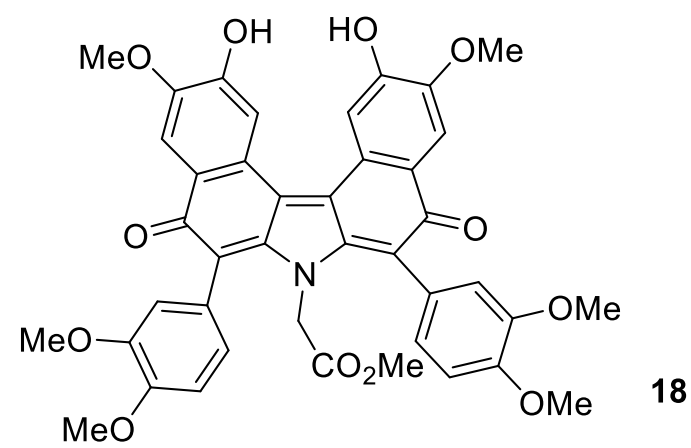

18 (Methyl-(6,8-bis(3,4-dimethoxyphenyl)-2,12-dihydroxy-3,11-dimethoxy-5,9 -dioxo-5H-dibenzo [c,g]carbazol-7(9H)-yl)acetate): Brown solid, IR $v_{\max } 2925$, 1748, $1287 \mathrm{~cm}^{-1} ;{ }^{1} \mathrm{H} \mathrm{NMR}\left(\mathrm{CDCl}_{3}, 400 \mathrm{MHz}\right) \delta_{\mathrm{H}} 7.94(\mathrm{~s}, 2 \mathrm{H}), 7.56(\mathrm{~s}, 2 \mathrm{H}), 6.84(\mathrm{~d}, J$ $=8.5 \mathrm{~Hz}, 2 \mathrm{H}), 6.75-6.74(\mathrm{~m}, 4 \mathrm{H}), 6.65(\mathrm{bs}, 2 \mathrm{H}), 3.93(\mathrm{~s}, 6 \mathrm{H}), 3.87(\mathrm{~s}, 6 \mathrm{H}), 3.83(\mathrm{~s}$, 6H), $3.70(\mathrm{bs}, 2 \mathrm{H}), 3.37(\mathrm{~s}, 3 \mathrm{H}) ;{ }^{13} \mathrm{C} \mathrm{NMR}\left(\mathrm{CDCl}_{3}, 100 \mathrm{MHz}\right) \delta_{\mathrm{C}} 183.7,169.1,153.5$, 149.2 , 148.9, 148.8, 130.7, 129.0, 125.3, 125.1, 124.6, 123.4, 117.2, 114.2, 112.4, 111.1, 109.7, 56.3, 56.0, 56.0, 51.8, 47.4; HRESIMS $m / z 734.0571[\mathrm{M}+\mathrm{H}]^{+}$(calcd for $^{2}$ $\mathrm{C}_{41} \mathrm{H}_{36} \mathrm{NO}_{12}$ 734.2159).

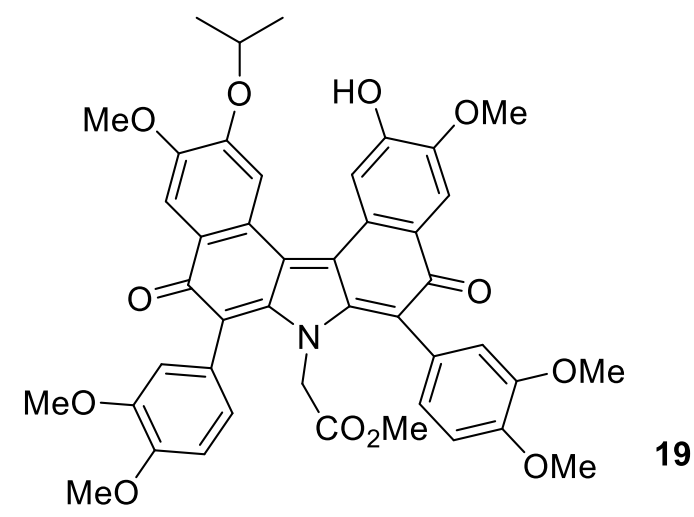

19 (Methyl-2-(6,8-bis(3,4-dimethoxyphenyl)-2-hydroxy-12-isopropoxy-3,11 -dimethoxy-5,9-dioxo-5H- dibenzo[c,g]carbazol-7(9H)-yl)acetate): Brown solid, IR $v_{\max } 2931,2848,1748,1263,1021 \mathrm{~cm}^{-1} ;{ }^{1} \mathrm{H} \mathrm{NMR}\left(\mathrm{CDCl}_{3}, 400 \mathrm{MHz}\right) \delta_{\mathrm{H}} 7.97(\mathrm{~s}, 1 \mathrm{H})$, $7.91(\mathrm{~s}, 1 \mathrm{H}), 7.67(\mathrm{~s}, 1 \mathrm{H}), 7.64(\mathrm{~s}, 1 \mathrm{H}), 6.85(\mathrm{~d}, J=8.2 \mathrm{~Hz}, 2 \mathrm{H}), 6.76-6.73(\mathrm{~m}, 4 \mathrm{H})$, $6.21(\mathrm{bs}, 1 \mathrm{H}), 4.63-4.57(\mathrm{~m}, 1 \mathrm{H}), 4.01(\mathrm{~s}, 3 \mathrm{H}), 3.96(\mathrm{~s}, 3 \mathrm{H}), 3.88(\mathrm{~s}, 6 \mathrm{H}), 3.83(\mathrm{~s}, 6 \mathrm{H})$, $3.70(\mathrm{bs}, 2 \mathrm{H}), 3.37(\mathrm{~s}, 3 \mathrm{H}), 1.42(\mathrm{~d}, J=6.1 \mathrm{~Hz}, 6 \mathrm{H}) ;{ }^{13} \mathrm{C} \mathrm{NMR}\left(\mathrm{CDCl}_{3}, 100 \mathrm{MHz}\right) \delta_{\mathrm{C}}$ $183.7,183.6,169.0,153.5,152.1,150.2,149.1,148.8,148.6,148.5,131.3,130.0$, $125.5,125.2,125.1,124.9,123.9,123.4,117.3,117.2,114.1,112.1,111.8,111.0$, $110.3,109.7,72.1,69.7,56.4,56.3,56.0,55.9,53.9,51.8,47.4,22.3$; HRESIMS $m / z$ 
$776.0617[\mathrm{M}+\mathrm{H}]^{+}$(calcd for $\mathrm{C}_{44} \mathrm{H}_{42} \mathrm{NO}_{12}$ 776.2629).
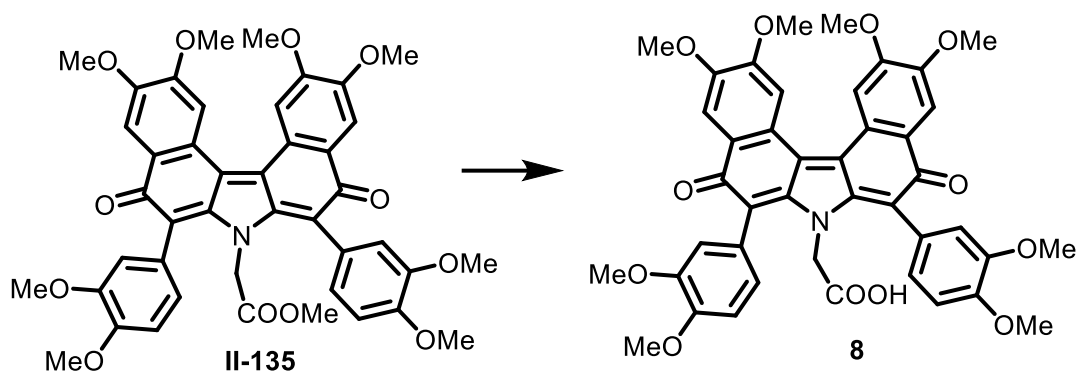

\section{Scheme S9. Conversion of II-135 to 8}

Synthesis of 8: Lithium hydroxide (396 mg, $9.4 \mathrm{mmol}, 40$ eq.) was added to II-135 (200 mg, 1.0 eq.) in tetrahydrofuran $-\mathrm{H}_{2} \mathrm{O}(20 \mathrm{~mL}, 1 / 1, \mathrm{v} / \mathrm{v})$. The mixture was stirred at $60{ }^{\circ} \mathrm{C}$ for two days. After cooling to rt, the $\mathrm{pH}$ was adjusted to $4-5$ with $6 \%$ $\mathrm{HCl}$ solution, and the solution was extracted with $\mathrm{CH}_{2} \mathrm{Cl}_{2}(3 \times 60 \mathrm{~mL})$. The organic layer was washed with brine $(100 \mathrm{~mL})$ and then concentrated under vacuo. The residue was purified by silica gel column eluting with $2.5 \% \mathrm{EtOH}-\mathrm{CH}_{2} \mathrm{Cl}_{2}$ containing $0.2 \% \mathrm{CF}_{3} \mathrm{COOH}$ to yield 8 (145 $\mathrm{mg}$, yield $\left.82 \%\right)$.

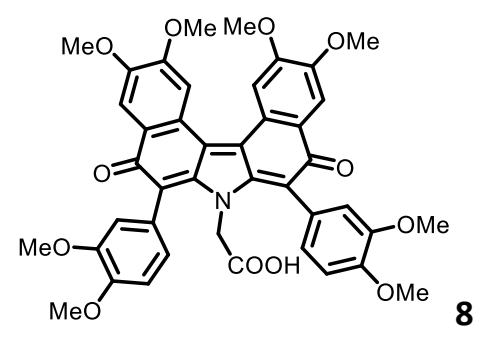

8 (2-(6,8-bis(3,4-dimethoxyphenyl)-2,3,11,12-tetramethoxy-5,9-dioxo-5H-

dibenzo[c,g]carbazol-7(9H)-yl)acetic acid): Dark red solid, IR $v_{\max }$ 3495, 2928, 1725, $1264 \mathrm{~cm}^{-1} ;{ }^{1} \mathrm{H} \mathrm{NMR}\left(\mathrm{CDCl}_{3}, 400 \mathrm{MHz}\right) \delta_{\mathrm{H}} 7.91(\mathrm{~s}, 2 \mathrm{H}), 7.64(\mathrm{~s}, 2 \mathrm{H}), 6.83(\mathrm{~d}, J$ $=8.0 \mathrm{~Hz}, 2 \mathrm{H}), 6.75-6.71(\mathrm{~m}, 4 \mathrm{H}), 3.99(\mathrm{~s}, 6 \mathrm{H}), 3.87(\mathrm{~s}, 6 \mathrm{H}), 3.84(\mathrm{~s}, 6 \mathrm{H}), 3.77(\mathrm{~s}, 6 \mathrm{H})$, $3.68(\mathrm{bs}, 2 \mathrm{H}) ;{ }^{13} \mathrm{C} \mathrm{NMR}\left(\mathrm{CDCl}_{3}, 100 \mathrm{MHz}\right) \delta_{\mathrm{C}} 184.1,153.9,151.6,151.4,149.4$, 149.0, 132.9, 131.0, 126.0, 124.4, 124.2, 123.4, 117.6, 113.9, 111.4, 110.1, 109.0, 56.7, 56.4, 56.0, 56.0, 46.9; HRESIMS $m / z 746.2217$ [M-H] $]^{-}$(calcd for $\mathrm{C}_{42} \mathrm{H}_{36} \mathrm{NO}_{12}$ 746.2316). 

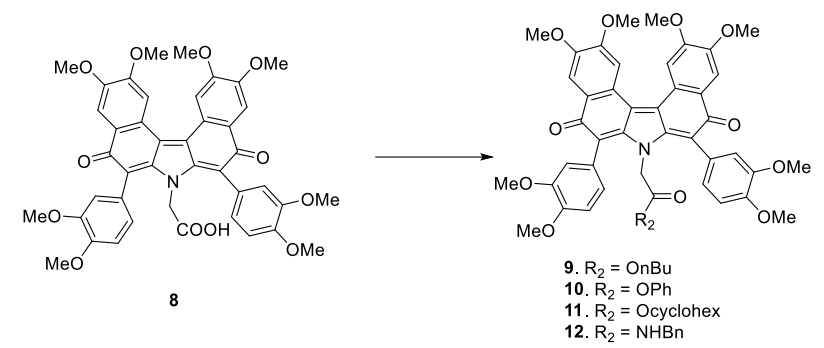

\section{Scheme S10. Conversion of 8 to 9-12}

Conversion of 8 to $9-12$. Dicyclohexylcarbodiimide $(1.1$ eq. $)$, 4-dimethylaminopyridine (0.1 eq.) and $n$ - $\mathrm{BuOH}$ (2.5 eq.) were added to a solution of 8 (1.0 eq.) in dry $\mathrm{CH}_{2} \mathrm{Cl}_{2}$ under $\mathrm{N}_{2}$. The mixture was stirred at $\mathrm{rt}$ for $16 \mathrm{~h}$. The concentrated residue was purified by silica gel column eluting with $\mathrm{CH}_{2} \mathrm{Cl}_{2}-\mathrm{MeOH}$ (5:1) to obtain 9 (yield 33\%). Compounds 10 (yield 36\%), 11 (yield 29\%) and 12 (yield 57\%) were derived from $\mathbf{8}$ by adding phenol, cyclohexanol, or amine respectively in the same method as for 9.

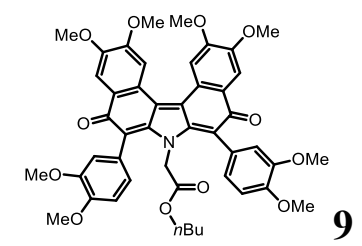

\section{9 (Butyl-2-(6,8-bis(3,4-dimethoxyphenyl)-2,3,11,12-tetramethoxy-5,9-dioxo-} 5H-dibenzo[c,g]carbazol-7(9H)-yl)acetate): Dark red solid, IR $v_{\max }$ 2935, 2836, 1750, 1269, $1022 \mathrm{~cm}^{-1} ;{ }^{1} \mathrm{H}$ NMR $\left(\mathrm{CDCl}_{3}, 400 \mathrm{MHz}\right) \delta_{\mathrm{H}} 7.92(\mathrm{~s}, 2 \mathrm{H}), 7.66(\mathrm{~s}, 2 \mathrm{H})$, $6.85(\mathrm{~d}, J=8.2 \mathrm{~Hz}, 2 \mathrm{H}), 6.78-6.75(\mathrm{~m}, 4 \mathrm{H}), 4.00(\mathrm{~s}, 6 \mathrm{H}), 3.88(\mathrm{~s}, 6 \mathrm{H}), 3.87(\mathrm{~s}, 6 \mathrm{H})$, $3.83(\mathrm{bs}, 6 \mathrm{H}), 3.78-3.66(\mathrm{~m}, 4 \mathrm{H}), 1.36-1.29(\mathrm{~m}, 2 \mathrm{H}), 1.19-1.09(\mathrm{~m}, 2 \mathrm{H}), 0.81(\mathrm{t}, J=$ $7.3 \mathrm{~Hz}, 3 \mathrm{H}) ;{ }^{13} \mathrm{C} \mathrm{NMR}\left(\mathrm{CDCl}_{3}, 100 \mathrm{MHz}\right) \delta_{\mathrm{C}} 183.6,168.5,153.7,151.3,151.3,149.2$, $148.8,130.7,129.6,126.2,124.9,124.2,117.4,115.1,111.0,109.9,108.9,65.1,56.6$, 56.3, 55.9, 47.5, 30.3, 19.0, 13.7; HRESIMS $m / z 804.3013[\mathrm{M}+\mathrm{H}]^{+}$(calcd for $\mathrm{C}_{46} \mathrm{H}_{46} \mathrm{NO}_{12}$ 804.2942).

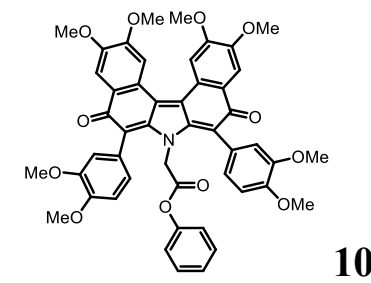


-5H-dibenzo[c,g] carbazol-7(9H)-yl)acetate): Dark red solid, IR $v_{\max }$ 2951, 1751, $1270 \mathrm{~cm}^{-1} ;{ }^{1} \mathrm{H} \mathrm{NMR}\left(\mathrm{CDCl}_{3}, 400 \mathrm{MHz}\right) \delta_{\mathrm{H}} 7.92(\mathrm{~s}, 2 \mathrm{H}), 7.66(\mathrm{~s}, 2 \mathrm{H}), 7.30(\mathrm{~d}, J=7.7$ $\mathrm{Hz}, 2 \mathrm{H}), 7.21-7.14(\mathrm{~m}, 2 \mathrm{H}), 6.88-6.78(\mathrm{~m}, 7 \mathrm{H}), 4.03(\mathrm{~s}, 6 \mathrm{H}), 3.86-3.76(\mathrm{~m}, 18 \mathrm{H})$, 3.72-3.68 (m, 2H); ${ }^{13} \mathrm{C} \mathrm{NMR}\left(\mathrm{CDCl}_{3}, 100 \mathrm{MHz}\right) \delta_{\mathrm{C}} 183.7,166.7,153.5,151.4,151.3$, 149.9, 149.3, 148.9, 130.7, 129.7, 129.4, 126.1, 125.0, 124.2, 120.9, 120.5, 117.4, 115.4, 111.2, 109.9, 108.9, 56.6, 56.3, 56.0, 55.9, 47.7; HRESIMS m/z 824.2694 $[\mathrm{M}+\mathrm{H}]^{+}$(calcd for $\mathrm{C}_{48} \mathrm{H}_{42} \mathrm{NO}_{12}$ 824.2629).

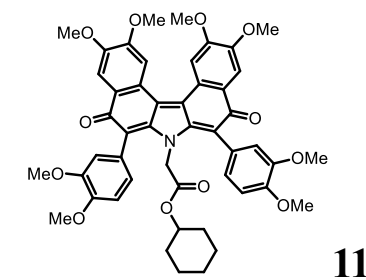

11 (Cyclohexyl-2-(6,8-bis(3,4-dimethoxyphenyl)-2,3,11,12-tetramethoxy-5,9 -dioxo-5H-dibenzo[c,g] carbazol-7(9H)-yl)acetate): Dark red solid, IR $v_{\max } 2951$, 2837, 1751, 1270, $1022 \mathrm{~cm}^{-1} ;{ }^{1} \mathrm{H}$ NMR $\left(\mathrm{CDCl}_{3}, 400 \mathrm{MHz}\right) \delta_{\mathrm{H}} 7.92(\mathrm{~s}, 2 \mathrm{H}), 7.67$ (s, 2H), 6.91-6.77 (m, 6H), $4.46(\mathrm{bs}, 1 \mathrm{H}), 4.00(\mathrm{~s}, 6 \mathrm{H}), 3.89(\mathrm{~s}, 6 \mathrm{H}), 3.87(\mathrm{~s}, 6 \mathrm{H}), 3.84$ (bs, 6H), 3.76-3.69 (m, 2H), 1.58-1.43 (m, 4H), 1.14-1.06 (m, 4H), 0.89-0.83 (m, 2H); ${ }^{13} \mathrm{C} \mathrm{NMR}\left(\mathrm{CDCl}_{3}, 100 \mathrm{MHz}\right) \delta_{\mathrm{C}} 183.6,167.8,154.1,151.3,151.3,149.2,148.9$, 129.7, 126.3, 125.1, 124.2, 120.8, 117.5, 115.4, 111.2, 110.0, 108.9, 73.9, 56.7, 56.4, 56.0, 31.3, 25.2, 23.4; HRESIMS $m / z 830.3160[\mathrm{M}+\mathrm{H}]^{+}$(calcd for $\mathrm{C}_{48} \mathrm{H}_{48} \mathrm{NO}_{12}$ 830.3098).

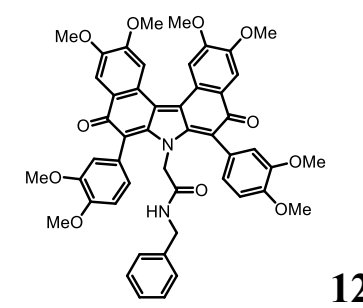

12 (N-benzyl-2-(6,8-bis(3,4-dimethoxyphenyl)-2,3,11,12-tetramethoxy-5,9dioxo-5H-dibenzo[c,g]carbazol-7(9H)-yl)acetamide): Dark red solid, IR $v_{\max } 2934$, 2837, 1751, 1584, 1269, $1023 \mathrm{~cm}^{-1} ;{ }^{1} \mathrm{H} \mathrm{NMR}\left(\mathrm{CDCl}_{3}, 400 \mathrm{MHz}\right) \delta_{\mathrm{H}} 7.96(\mathrm{~s}, 2 \mathrm{H}), 7.69$ (s, 2H), 7.34-7.30 (m, 3H), $7.12(\mathrm{~d}, J=6.4 \mathrm{~Hz}, 2 \mathrm{H}), 6.84-6.74(\mathrm{~m}, 6 \mathrm{H}), 4.57$ (bs, 1H), 4.21-4.08 (m, 2H), $4.03(\mathrm{~s}, 6 \mathrm{H}), 3.90(\mathrm{~s}, 6 \mathrm{H}), 3.86(\mathrm{bs}, 6 \mathrm{H}), 3.84(\mathrm{~s}, 6 \mathrm{H}), 3.62-3.58(\mathrm{~m}$, $2 \mathrm{H}) ;{ }^{13} \mathrm{C} \mathrm{NMR}\left(\mathrm{CDCl}_{3}, 100 \mathrm{MHz}\right) \delta_{\mathrm{C}} 183.6,166.9,154.4,151.3,151.3,149.03,148.8$, 
$137.4,130.9,128.9,128.3,128.0,126.3,125.5,125.4,124.3,117.2,114.3,111.0$, 109.9, 109.1, 56.7, 56.4, 56.0, 55.9, 48.8, 43.7; HRESIMS $m / z$ 837.3019 $[\mathrm{M}+\mathrm{H}]^{+}$ (calcd for $\mathrm{C}_{49} \mathrm{H}_{45} \mathrm{~N}_{2} \mathrm{O}_{11} 837.2945$ ).

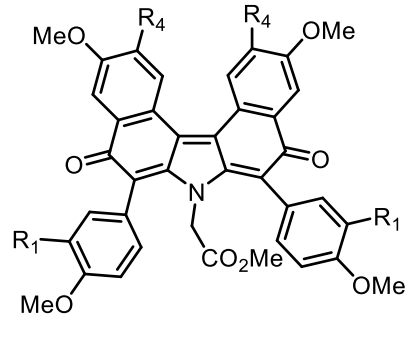

$\mathrm{R}_{1}=\mathrm{OH}, \mathrm{R}_{4}=\mathrm{OMe}: \mathbf{6}$ $\mathrm{R}_{1}=\mathrm{OMe}, \mathrm{R}_{4}=\mathrm{OH}: 18$

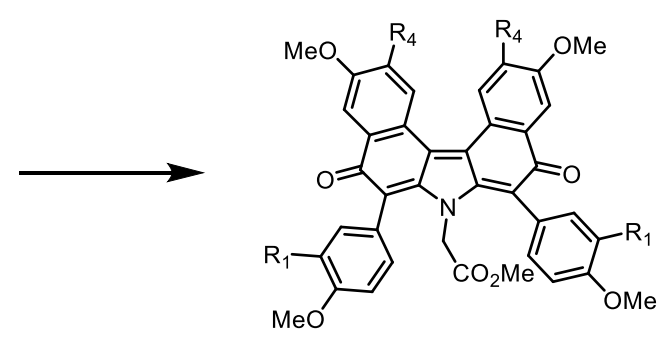

$\mathrm{R}_{1}=\mathrm{OAc}, \mathrm{R}_{4}=\mathrm{OMe}: 7$ $\mathrm{R}_{1}=\mathrm{OMe}, \mathrm{R}_{4}=\mathrm{OAC}: 20$

Scheme S11. Synthesis of 7 and 20

Synthesis of 7 and 20. Acetic anhydride (3.0 eq.) and pyridine (4.0 eq.) were added to 6 (1.0 eq.) in dry $\mathrm{CH}_{2} \mathrm{Cl}_{2}$ under $\mathrm{N}_{2}$. The mixture was stirred at $\mathrm{rt}$ for $22 \mathrm{~h}$. $\mathrm{H}_{2} \mathrm{O}$ was then added, while the mixture was extracted with $\mathrm{CH}_{2} \mathrm{Cl}_{2}$. The organic layer was washed with brine and concentrated under vacuo. The residue was purified by silica gel column eluting with hexane-acetone (1:1) to yield 7 (yield 90\%). Compound $\mathbf{2 0}$ (yield 60\%) was derived from 18 by the same protocol as for 7.

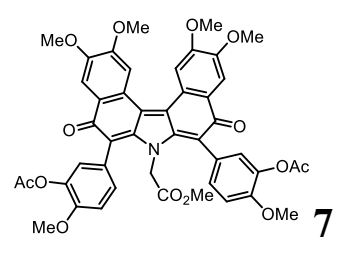

7 ((2,3,11,12-tetramethoxy-7-(2-methoxy-2-oxoethyl)-5,9-dioxo-7,9-dihydro -5H-dibenzo[c,g]carbazole-6,8-diyl)bis(2-methoxy-5,1-phenylene)diacetate): Dark red solid, IR $v_{\max } 2918,, 1764,1263 \mathrm{~cm}^{-1} ;{ }^{1} \mathrm{H}$ NMR $\left(\mathrm{CDCl}_{3}, 400 \mathrm{MHz}\right) \delta_{\mathrm{H}} 7.91(\mathrm{~s}$, 2H), $7.65(\mathrm{~s}, 2 \mathrm{H}), 7.08(\mathrm{~d}, J=7.3 \mathrm{~Hz}, 2 \mathrm{H}), 6.98-6.95(\mathrm{~m}, 4 \mathrm{H}), 3.99(\mathrm{~s}, 6 \mathrm{H}), 3.86(\mathrm{~s}$, 6H), 3.85 (s, 6H), $3.78(\mathrm{bs}, 2 \mathrm{H}), 3.39$ (s, 3H), $2.28(\mathrm{~s}, 6 \mathrm{H}) ;{ }^{13} \mathrm{C} \mathrm{NMR}\left(\mathrm{CDCl}_{3}, 100\right.$ $\mathrm{MHz}) \delta_{\mathrm{C}} 183.1,168.6,168.5,151.3,151.2,139.7,130.8,130.8,129.4,129.3,126.1$, $125.7,124.7,124.2,116.4,112.2,109.9,108.9,56.6,56.3,56.0,52.1,48.0,20.8$ HRESIMS $m / z$ 818.2441 [M+H] $]^{+}$(calcd for $\mathrm{C}_{45} \mathrm{H}_{40} \mathrm{NO}_{14}$ 818.2371).

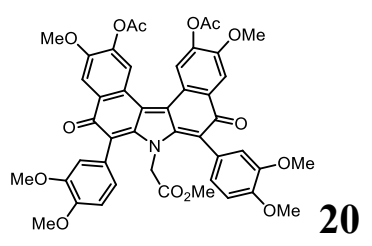




\section{0 (6,8-bis(3,4-dimethoxyphenyl)-3,11-dimethoxy-7-(2-methoxy-2-oxoethyl)}

-5,9-dioxo-7,9-dihydro-5H-dibenzo[c,g]carbazole-2,12-diyldiacetate): Dark red solid, IR v $2930\left(v_{\text {as C-H }}\right), 2850\left(v_{\mathrm{s} \text { C-H}}\right), 1755\left(v_{\mathrm{C}=\mathrm{O}}\right), 1748\left(v_{\mathrm{C}=\mathrm{O}}\right), 1269$ ( $\left.v_{\text {as C-O-C }}\right), 1015$ $\left(v_{\mathrm{s} \mathrm{C}-\mathrm{O}-\mathrm{C}}\right) \mathrm{cm}^{-1} ;{ }^{1} \mathrm{H} \mathrm{NMR}\left(\mathrm{CDCl}_{3}, 400 \mathrm{MHz}\right) \delta 8.09(\mathrm{~s}, 2 \mathrm{H}), 7.78(\mathrm{~s}, 2 \mathrm{H}), 6.88(\mathrm{~d}, J=$ 8.1 Hz, 2H), 6.78-6.75 (m, 4H), 3.97 (s, 6H), $3.91(\mathrm{~s}, 6 \mathrm{H}), 3.86(\mathrm{~s}, 6 \mathrm{H}), 3.79-3.73(\mathrm{~m}$, 2H), $3.40(\mathrm{~s}, 3 \mathrm{H}), 2.39$ (s, 6H); ${ }^{13} \mathrm{C} \mathrm{NMR}\left(\mathrm{CDCl}_{3}, 100 \mathrm{MHz}\right) \delta 183.4,169.2,153.2$, $149.3,148.9,142.1,130.5,129.9,124.8,124.7,123.4,123.1,121.9,117.8,114.0$, 113.9, 111.2, 111.0, 56.4, 56.0, 56.0, 51.9, 47.3, 20.8; HRESIMS m/z 818.0909 $[\mathrm{M}+\mathrm{H}]^{+}\left(\right.$calcd for $\left.\mathrm{C}_{45} \mathrm{H}_{40} \mathrm{NO}_{14} 818.2371\right)$.

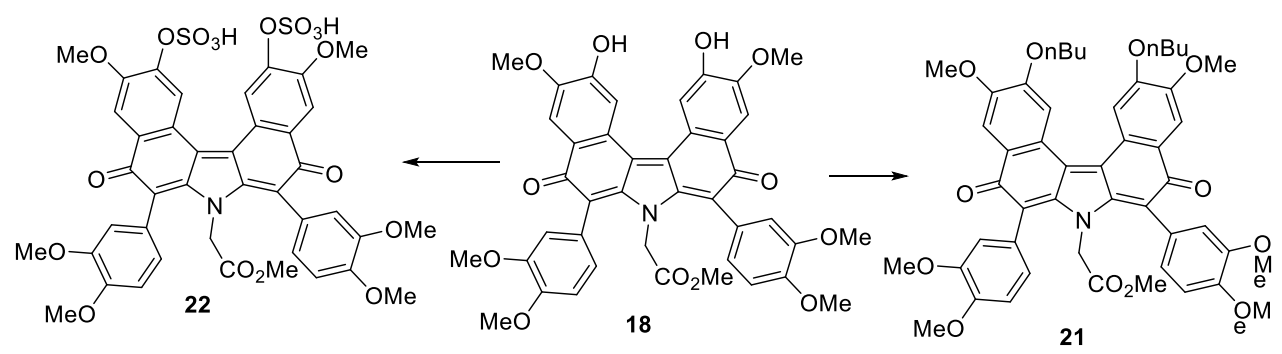

\section{Scheme S12. Conversion of 18 to 21 and 22}

Conversion of 18 to 21 and 22. Bromobutane $(7.8 \mu \mathrm{L}, 0.073 \mathrm{mmol})$ and $\mathrm{K}_{2} \mathrm{CO}_{3}$ (16.7 $\mathrm{mg}, 0.12 \mathrm{mmol})$ were added to a solution of $18(17.8 \mathrm{mg}, 0.024 \mathrm{mmol})$ in dry $\mathrm{N}, \mathrm{N}$-dimethylformamide $(3.0 \mathrm{~mL})$ to stir at $50{ }^{\circ} \mathrm{C}$ overnight. After cooling to $\mathrm{rt}$ and adding $\mathrm{H}_{2} \mathrm{O}(15 \mathrm{~mL})$, the $\mathrm{pH}$ was adjusted to $7-8$ with $2 \mathrm{~N} \mathrm{HCl}$ solution. The mixture was then extracted with EtOAc $(3 \times 15 \mathrm{~mL})$ and the organic layer was washed with brine $(40 \mathrm{~mL})$ to concentrate under vacuo. The residue was purified by silica gel column eluting with EtOAc- $\mathrm{CH}_{2} \mathrm{Cl}_{2}$ (1:9) to yield 21 (8.0 mg, 39\% yield).

Chlorosulfonic acid (54 $\mu \mathrm{L}, 0.04 \mathrm{mmol}, 40$ eq.) was added slowly to a solution of 18 (15.0 mg, $0.02 \mathrm{mmol}, 1.0$ eq.) in anhydrous pyridine $(0.8 \mathrm{~mL})$ under $\mathrm{N}_{2}$ to stir at rt for $20 \mathrm{~h}$. After concentration under vacuo, it was dissolved in $\mathrm{H}_{2} \mathrm{O}(20 \mathrm{~mL})$ to adjust $\mathrm{pH}$ to $3-4$ by $2 \mathrm{~N} \mathrm{HCl}$. The mixture was then extracted with EtOAc $(3 \times 30 \mathrm{~mL})$ and was purified by silica gel column eluting with $\mathrm{MeOH}-\mathrm{CH}_{2} \mathrm{Cl}_{2}$ (3:7) to yield 22 (4.5 $\mathrm{mg}, 25 \%$ yield). 


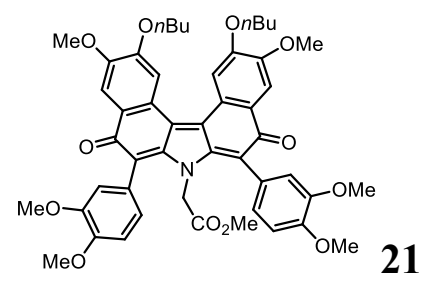

21 (Methyl-2-(2,12-dibutoxy-6,8-bis(3,4-dimethoxyphenyl)-3,11-dimethoxy -5,9-dioxo-5H-dibenzo [c,g] carbazol-7(9H)-yl)acetate): Dark red solid, IR $v_{\max }$ 2918, 1756, $1264 \mathrm{~cm}^{-1} ;{ }^{1} \mathrm{H} \mathrm{NMR}\left(\mathrm{CDCl}_{3}, 400 \mathrm{MHz}\right) \delta_{\mathrm{H}} 7.91(\mathrm{~s}, 2 \mathrm{H}), 7.65$ (s, 2H), $6.85(\mathrm{~d}, J=8.2 \mathrm{~Hz}, 2 \mathrm{H}), 6.77-6.73(\mathrm{~m}, 4 \mathrm{H}), 3.99-3.96(\mathrm{~m}, 10 \mathrm{H}), 3.88(\mathrm{~s}, 6 \mathrm{H}), 3.83(\mathrm{~s}$, $6 \mathrm{H}), 3.70(\mathrm{bs}, 2 \mathrm{H}), 3.37(\mathrm{~s}, 3 \mathrm{H}), 1.84-1.76(\mathrm{~m}, 4 \mathrm{H}), 1.51-1.42(\mathrm{~m}, 4 \mathrm{H}), 0.94(\mathrm{t}, J=7.4$ $\mathrm{Hz}, 6 \mathrm{H}) ;{ }^{13} \mathrm{C} \mathrm{NMR}\left(\mathrm{CDCl}_{3}, 100 \mathrm{MHz}\right) \delta_{\mathrm{C}} 183.7,169.0,151.6,151.1,149.2,148.8$, 130.6, 125.9, 125.1, 124.1, 123.4, 123.4, 117.2, 114.0, 111.0, 110.2, 110.1, 69.5, 56.30, 56.0, 55.9, 51.8, 47.3, 31.2, 19.4, 13.9; HRESIMS $m / z$ 846.1692 [M+H] ${ }^{+}$(calcd for $\mathrm{C}_{49} \mathrm{H}_{52} \mathrm{NO}_{12}$ 846.3411).

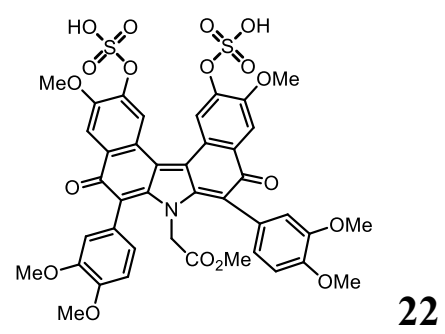

22 (Methyl-2-(6,8-bis(3,4-dimethoxyphenyl)-3,11-dimethoxy-5,9-dioxo-2, 12-bis(sulfooxy)-5H-dibenzo [c,g]carbazol-7(9H)-yl)acetate): Dark red solid, IR $v_{\max } 2921,2851,1045 \mathrm{~cm}^{-1} ;{ }^{1} \mathrm{H}$ NMR $\left(\mathrm{CD}_{3} \mathrm{OD}, 400 \mathrm{MHz}\right) \delta_{\mathrm{H}} 8.55$ (s, 2H), $7.70(\mathrm{~s}$, 2H), $6.97(\mathrm{~d}, J=8.1 \mathrm{~Hz}, 2 \mathrm{H}), 6.79-6.74(\mathrm{~m}, 4 \mathrm{H}), 3.97(\mathrm{~s}, 6 \mathrm{H}), 3.85(\mathrm{~s}, 6 \mathrm{H}), 3.81(\mathrm{~s}$, 6H), 3.69 (bs, 2H), 3.39 (s, 3H); $\left.{ }^{13} \mathrm{C} \mathrm{NMR} \mathrm{(CD}{ }_{3} \mathrm{OD}, 100 \mathrm{MHz}\right) \delta_{\mathrm{C}} 185.3,169.5,155.3$, $150.6,150.2,145.6,131.4,130.2,126.3,124.8,124.3,122.3,118.9,116.0,115.9$, 112.6, 111.9, 56.6, 56.5, 56.5, 52.4, 50.1; HRESIMS $m / z$ 445.5297 [M-2H] $]^{2-}$ (calcd for $\left.\mathrm{C}_{41} \mathrm{H}_{33} \mathrm{NO}_{18} \mathrm{~S}_{2} 891.1296\right)$. 

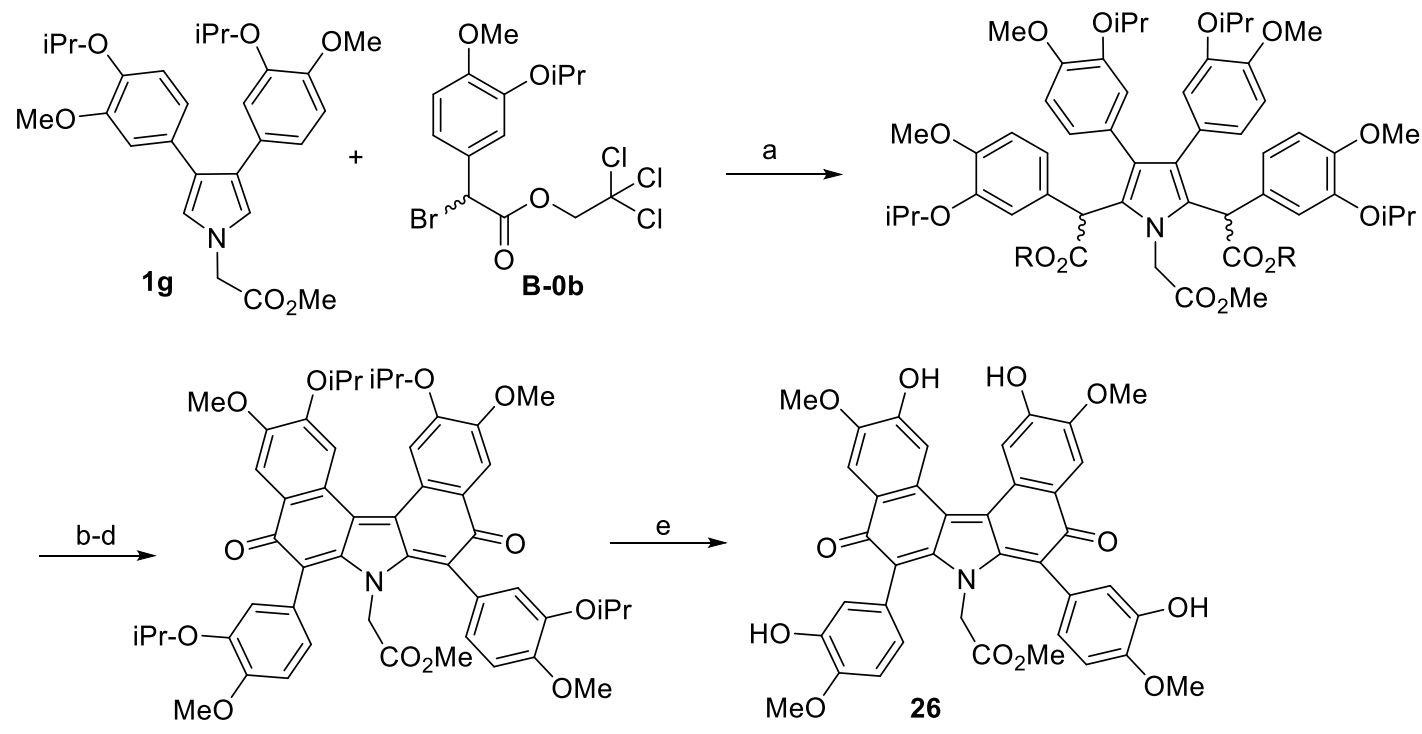

a) $\mathrm{Al}_{2} \mathrm{O}_{3}\left(\mathrm{H}^{+}\right), \mathrm{CH}_{2} \mathrm{Cl}_{2}$, reflux,10h; b) $\mathrm{Zn}, \mathrm{NH}_{4} \mathrm{OAc}, \mathrm{THF}$, rt, $1.5 \mathrm{~h}$; c) $\mathrm{Ac}_{2} \mathrm{O}, \mathrm{KOAc}$, reflux, $1.5 \mathrm{~h}$; d) aq. $\mathrm{NaOH}$, $\mathrm{MeOH}$, air, 55oC, $0.5 \mathrm{~h} ; \mathrm{e}) \mathrm{AlCl}_{3}, \mathrm{CH}_{2} \mathrm{Cl}_{2}, \mathrm{rt}, 16 \mathrm{~h}$

\section{Scheme S13. Synthesis of 26}

Synthesis of 26. Conversion of $\mathbf{1 g}$ to $\mathbf{2 6}$ was followed by the same protocol as for the synthesis of II-135, while hydrolysis of 17 a to 6 a was performed by the same protocol for the conversion of 5 to 6.

26: Brown solid, IR $v_{\max } 2919,1745,1250 \mathrm{~cm}^{-1} ;{ }^{1} \mathrm{H} \mathrm{NMR}\left(\mathrm{CDCl}_{3}, 400 \mathrm{MHz}\right) \delta_{\mathrm{H}}$ $7.90(\mathrm{~s}, 2 \mathrm{H}), 7.66(\mathrm{~s}, 2 \mathrm{H}), 6.81(\mathrm{~d}, J=8.2 \mathrm{~Hz}, 2 \mathrm{H}), 6.74(\mathrm{~s}, 2 \mathrm{H}), 6.64(\mathrm{~d}, J=8.2 \mathrm{~Hz}$, 2H), $5.60(\mathrm{~s}, 2 \mathrm{H}), 4.00(\mathrm{~s}, 6 \mathrm{H}), 3.90(\mathrm{~s}, 6 \mathrm{H}), 3.65(\mathrm{bs}, 2 \mathrm{H}), 3.46(\mathrm{~s}, 3 \mathrm{H}) ;{ }^{13} \mathrm{C} \mathrm{NMR}$ $\left(\mathrm{CDCl}_{3}, 100 \mathrm{MHz}\right) \delta_{\mathrm{C}} 183.4,168.6,153.2,151.0,151.1,146.5,145.5,145.4,126.1$, $125.7,125.7,124.2,117.1,117.1,110.5,109.9,108.9,56.5 / 55.4,56.0 / 56.2,52.2,47.8$; HRESIMS $m / z$ 706.1921 $[\mathrm{M}+\mathrm{H}]^{+}$. 


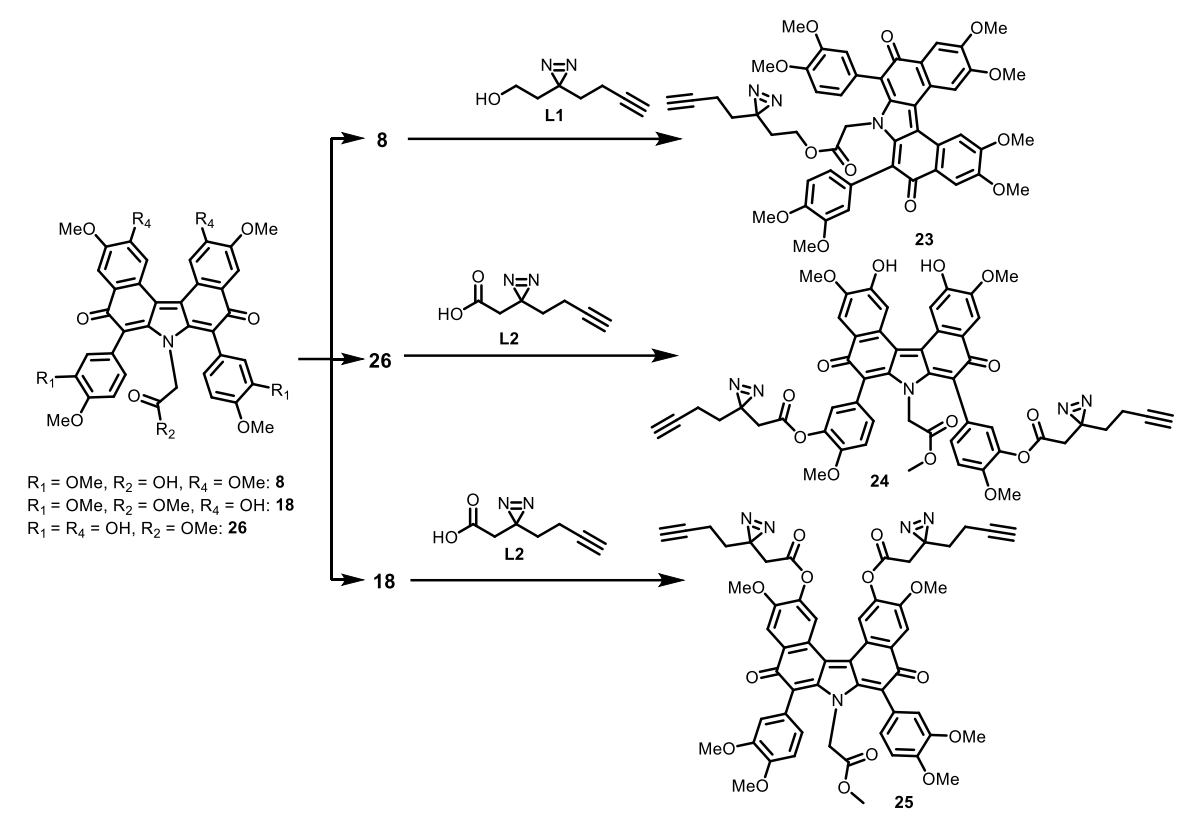

Scheme S14. Synthesis of probes 23 to 25

Dicyclohexylcarbodiimide (1.1 eq.), 4-dimethylaminopyridine (0.1 eq.) and L1 (1.5 eq.) were added to a solution of 8 (1.0 eq.) in dry $\mathrm{CH}_{2} \mathrm{Cl}_{2}$ under $\mathrm{N}_{2}$ to stir at $\mathrm{rt}$ for $16 \mathrm{~h}$. The residue was purified by silica gel column eluting with hexane-acetone $2: 1$ to yield 23 (yield 65\%). Probes 24 (yield 91\%) and $\mathbf{2 5}$ (yield 45\%) were derived from $\mathbf{2 6}$ and 18 (1.0 eq.) respectively by the same protocol as $\mathbf{8}$. It is noted that the reaction of $\mathbf{2 6}$ with L2 was controlled within $5 \mathrm{~h}$ to produce $\mathbf{2 4}$ as a main product.

23 (2-(3-(but-3-yn-1-yl)-3H-diazirin-3-yl)ethyl2-(6,8-bis(3,4-dimethoxyphenyl)

\section{-2,3,11,12-tetramethoxy-5,9-dioxo-5H-dibenzo[c,g]carbazol-7(9H)-yl)acetate):}

Dark red solid, IR $v_{\max } 2928,1746,1267 \mathrm{~cm}^{-1} ;{ }^{1} \mathrm{H} \mathrm{NMR}\left(\mathrm{CDCl}_{3}, 400 \mathrm{MHz}\right) \delta_{\mathrm{H}} 7.91(\mathrm{~s}$, 2H), $7.64(\mathrm{~s}, 2 \mathrm{H}), 6.86(\mathrm{~d}, J=8.0 \mathrm{~Hz}, 2 \mathrm{H}), 6.77-6.73(\mathrm{~m}, 4 \mathrm{H}), 3.99(\mathrm{~s}, 6 \mathrm{H}), 3.88(\mathrm{~s}$, 6H), 3.86 (s, 6H), 3.82 (s, 6H), 3.74 (bs, 2H), 3.60 (bs, 2H), 1.94-1.93 (m, 1H), 1.87 $(\mathrm{td}, J=7.3,2.4 \mathrm{~Hz}, 2 \mathrm{H}), 1.46-1.42(\mathrm{~m}, 4 \mathrm{H}) ;{ }^{13} \mathrm{C} \mathrm{NMR}\left(\mathrm{CDCl}_{3}, 100 \mathrm{MHz}\right) \delta_{\mathrm{C}} 183.5$, $168.1,151.3,151.2,149.1,148.7,130.5,126.1,124.8,124.8,124.1,123.3,117.3$, $114.1,111.0,109.9,108.8,82.5,69.4,59.6,56.6,56.3,56.0,55.9,47.4,31.8,29.8$, 25.9, 13.1; HRESIMS $m / z$ 868.3067 [M+H] ${ }^{+}$(calcd. for $\mathrm{C}_{49} \mathrm{H}_{46} \mathrm{~N}_{3} \mathrm{O}_{12}$ 868.3003).

\section{4 (2,3,11,12-tetramethoxy-7-(2-methoxy-2-oxoethyl)-5,9-dioxo-7,9-dihydro} -5H-dibenzo[c,g]carbazole-6,8-diyl)bis(2-methoxy-5,1-phenylene) bis(2-(3-(but-3 -yn-1-yl)-3H- diazirin-3-yl)acetate)): Dark red solid, IR $v_{\max }$ 2922, 1766, 1715, $1268 \mathrm{~cm}^{-1} ;{ }^{1} \mathrm{H} \mathrm{NMR}\left(\mathrm{CDCl}_{3}, 400 \mathrm{MHz}\right) \delta_{\mathrm{C}} 7.92(\mathrm{~s}, 2 \mathrm{H}), 7.66(\mathrm{~s}, 2 \mathrm{H}), 7.12(\mathrm{~m}, 2 \mathrm{H})$, 
$7.01(\mathrm{~m}, 4 \mathrm{H}), 4.00(\mathrm{~s}, 6 \mathrm{H}), 3.88(\mathrm{~m}, 12 \mathrm{H}), 3.77(\mathrm{bs}, 2 \mathrm{H}), 3.44(\mathrm{~s}, 3 \mathrm{H}), 2.57(\mathrm{bs}, 4 \mathrm{H})$, 2.13-2.09 (m, 4H), $2.01(\mathrm{~m}, 2 \mathrm{H}), 1.88-1.85(\mathrm{~m}, 4 \mathrm{H}) ;{ }^{13} \mathrm{C} \mathrm{NMR}\left(\mathrm{CDCl}_{3}, 100 \mathrm{MHz}\right) \delta_{\mathrm{C}}$ $183.2,168.6,166.9,151.4,151.3,151.1,139.4,129.8,129.4,126.2,125.5,124.8$, 124.2, 116.3, 115.3, 112.3, 109.9, 108.9, 82.6, 69.7, 56.7, 56.4, 56.1, 52.3, 48.0, 39.6, 32.1, 25.7, 13.4; HRESIMS $m / z$ 974.2882 [M+H] ${ }^{+}$(calcd. for $\mathrm{C}_{52} \mathrm{H}_{44} \mathrm{~N}_{5} \mathrm{O}_{14}$ 974.2285).

\section{5 (6,8-bis(3,4-dimethoxyphenyl)-3,11-dimethoxy-7-(2-methoxy-2-oxoethyl)} -5,9-dioxo-7,9-dihydro-5H-dibenzo[c,g]carbazole-2,12-diyl-bis(2-(3-(but-3-yn-1-y I)- 3H-diazirin -3-yl)acetate)): Dark red solid, IR $v_{\max } 2917,1752,1268 \mathrm{~cm}^{-1} ;{ }^{1} \mathrm{H}$ NMR $\left(\mathrm{CDCl}_{3}, 400 \mathrm{MHz}\right) \delta_{\mathrm{H}} 8.08(\mathrm{~s}, 2 \mathrm{H}), 7.77(\mathrm{~s}, 2 \mathrm{H}), 6.87(\mathrm{~d}, J=8.0 \mathrm{~Hz}, 2 \mathrm{H})$, 6.77-6.73 (m, 4H), 3.97 (s, 6H), 3.89 (s, 6H), $3.84(\mathrm{~s}, 6 \mathrm{H}), 3.74(\mathrm{bs}, 2 \mathrm{H}), 3.39$ (s, 3H), $2.66(\mathrm{~s}, 4 \mathrm{H}), 2.15-2.10(\mathrm{~m}, 4 \mathrm{H}), 2.02(\mathrm{~m}, 2 \mathrm{H}), 1.91-1.88(\mathrm{~m}, 4 \mathrm{H}) ;{ }^{13} \mathrm{C} \mathrm{NMR}\left(\mathrm{CDCl}_{3}\right.$, $100 \mathrm{MHz}) \delta_{\mathrm{C}} 183.3,168.8,167.3,153.0,149.4,148.9,141.7,130.8,129.9,124.7$, 123.3, 123.1, 121.4, 117.9, 114.0, 113.9, 111.4, 111.1, 82.6, 69.6, 56.5, 56.1, 56.0, 51.9, 47.3, 40.0, 32.0, 25.7, 13.4; HRESIMS $m / z 1002.3155[\mathrm{M}+\mathrm{H}]^{+}$(calcd. for $\mathrm{C}_{55} \mathrm{H}_{48} \mathrm{~N}_{5} \mathrm{O}_{14}$ 1002.3120).

\section{Biology}

HIV-1 infectivity assay. To evaluate the inhibitory effects of baculiferins against HIV-1 infectivity, the experiments were carried out with the pseudotyped HIV-1 and TZM-bl indicator cells infected with HIV-1 strain SF33. For pseudotyped HIV-1, HEK293T cells were co-transfected with 300 ng pNL4-3Luc(R-E-), 200 ng VSVG plasmid DNA in 6-well-plates. After $48 \mathrm{~h}$ later, the supernatants were collected and filtered through a $0.45 \mu \mathrm{m}$ filter, and then used to infect the SupT1 cells $(1 \times 105)$ in 96-well plates. Different doses of baculiferins in DMSO (or Efavirenz as a positive control) were added to incubate for $48 \mathrm{~h}$, SupT1 cells were lysed and firefly luciferase activities were determined using a firefly Luciferase Assay System (Promega).

Photoaffinity labeling. H9 cells and H9 cells chronically infected with HIV-1 (H9-IIIB) in D-MEM with 10\% fetal bovine serum were incubated in a humidified $37{ }^{\circ} \mathrm{C}$ incubator with $5 \% \mathrm{CO}_{2}$ to reach $500 \mu \mathrm{L}, 0.5^{*} 10^{6}$ cells. To generate protein lysate, cells were washed twice with cold phosphate buffered saline (PBS), and were harvested by a cell scraper to collect by centrifugation. Cell pellets were re-suspended 
in lysis buffer (25 mM HEPES, pH 7.5, $150 \mathrm{mM} \mathrm{NaCl}, 2 \mathrm{mM} \mathrm{MgCl} 2,50 \mu \mathrm{M}$ PMSF) and lysed by sonication. Protein level was determined by the standard Bradford assay. Cell lysates were diluted with lysis buffer (25 mM HEPES, pH 7.5, $150 \mathrm{mM} \mathrm{NaCl}, 2$ $\mathrm{mM} \mathrm{MgCl} 2,50 \mu \mathrm{M}$ PMSF) to reach $5 \mathrm{mg} / \mathrm{mL}$ for labelling reactions and to store at $-80{ }^{\circ} \mathrm{C}$. The labelling reaction was initiated by incubation of lysate with the probe 23-25 $(100 \mu \mathrm{M})$ respectively in the presence (or not) of competitor $18(100 \mu \mathrm{M})$ at 0 ${ }^{\circ} \mathrm{C}$ for $1 \mathrm{~h}$. The mixture was then exposed to UV $365 \mathrm{~nm}(220 \mathrm{v}, 6 \mathrm{~W}, 365 \mathrm{~nm})$ at $0{ }^{\circ} \mathrm{C}$ for $30 \mathrm{~min}$ (the distance between the lamp and the lysate was kept about $3 \mathrm{~cm}$ ). Biotin- $\mathrm{N}_{3}(500 \mu \mathrm{M})$, sodium ascorbate aqueous solution $(1 \mathrm{mM})$ and copper (II)-TBTA complex $(1 \mathrm{mM})$ were added to incubate at $\mathrm{rt}$ in the dark for $2 \mathrm{~h}$. After the click reaction, cooled $\mathrm{MeOH}$ (4 folds of the lysate volume) was added and kept at -20 ${ }^{\circ} \mathrm{C}$ for overnight. The protein pellet was recovered by centrifugation $\left(13000 \mathrm{rpm}, 4{ }^{\circ} \mathrm{C}\right.$, $15 \mathrm{~min}$ ), and washed with cooled $\mathrm{MeOH}$ for twice in order to remove unreacted Biotin- $\mathrm{N}_{3}$. After evaporation of $\mathrm{MeOH}$ in air for $10 \mathrm{~min}$, the pellet was resolved in $0.1 \%$ SDS in PBS buffer with the help of ultrasound. After the addition of the protein loading buffer $(5 \times)$, the lysate was subjected to SDS-PAGE electrophoresis and then transferred onto polyvinylidene fluoride membrane. After blocking with 5\% BSA in TBST buffer, the membrane was incubated with streptavidin-HRP polymer conjugate (Bioss, cat bs-0437P-HPR). Biotinylated proteins were visualized by ChemiDoc $\mathrm{XRS}^{+}$system (Bio-Rad).

Enrichment of biotinylated protein and the identification by LC-MS/MS. H9 cells pellet washed by $\mathrm{MeOH}$ in photolabeling experiments was resolved in $0.4 \%$ SDS in PBS buffer. Prewashed streptavidin magnetic beads $(100 \mu \mathrm{L}$, Beijing ZhongKeLeiMing Daojin Technology Co., $1 \mu \mathrm{m}, 10 \mathrm{mg} / \mathrm{mL}$ ) were added. After the incubation at $\mathrm{rt}$ for $1 \mathrm{~h}$ on an orbital shaker, the beads were separated by a magnetic separator and washed successively with $200 \mu \mathrm{L}$ of buffer A (50 mM Tris-HCl pH 7.4; $0.1 \mathrm{mg} / \mathrm{mL}$ BSA), buffer B (50 mM Tris-HCl pH 7.4; $0.1 \mathrm{mg} / \mathrm{mL}$ BSA; 0.1\% SDS), buffer $\mathrm{C}(50 \mathrm{mM}$ Tris-HCl $\mathrm{pH} 7.4 ; 0.1 \mathrm{mg} / \mathrm{mL} \mathrm{BSA} ; 1 \mathrm{M} \mathrm{NaCl})$ and finally with distilled water. The beads were suspended in $0.1 \%$ SDS PBS buffer $(30 \mu \mathrm{L})$. After adding the protein loading buffer $(5 \times)$, the biotinylated proteins were then released 
from the beads by the incubation at $100{ }^{\circ} \mathrm{C}$ for $10 \mathrm{~min}$, then subjected to SDS-PAGE electrophoresis and visualized by silver stain (Sigma-Aldrich, ProteoSilver ${ }^{\mathrm{TM}}$ Plus Silver Stain Kit)[9]. Silver stained gel bands were excised and cut into $1.5 \mathrm{~mm}$ cubes. After washing by double distilled water $(50 \mu \mathrm{L}, 10 \mathrm{~min})$, gels were successively dehydrated with acetonitrile $(50 \mu \mathrm{L}, 5 \mathrm{~min})$, reduced with $10 \mathrm{mM}$ DTT $\left(20 \mu \mathrm{L}, 56^{\circ} \mathrm{C}\right.$, $1 \mathrm{~h}$ ), alkylated with $55 \mathrm{mM}$ iodoacetamide (20 $\mu \mathrm{L}$, in the dark, $45 \mathrm{~min}$ ), washed with $25 \mathrm{mM} \mathrm{NH}_{4} \mathrm{HCO}_{3}$ aqueous solution, and dehydrated with 50\% acetonitrile and acetonitrile respectively. Trypsin $\left(10 \mathrm{ng} / \mu \mathrm{L}\right.$ in $25 \mathrm{mM} \mathrm{NH}_{4} \mathrm{HCO}_{3}$ aqueous solution) was added and mixed. After incubating at $4{ }^{\circ} \mathrm{C}$ for $30 \mathrm{~min}$, the proteolysis proceeded for overnight at $37{ }^{\circ} \mathrm{C}$ and stopped by adding $2 \%$ TFA solution. Digested peptides were collected in the supernatant by centrifugation and acidified in $0.1 \%$ TFA.

LC-MS experiments were performed on a nano-flow HPLC system (Easy-nLC II, Thermo Fisher Scientific, Waltham, MA, USA) connected to a LTQ-Orbitrap Velos Pro mass spectrometer (Thermo Fisher Scientific), equipped with a Nanospray Flex Ion Source (Thermo Fisher Scientific). Peptide mixtures were injected at a flow rate of $5 \mu \mathrm{L} / \mathrm{min}$ onto a pre-column (Easy-column C18-A1, $100 \mu \mathrm{m} \mathrm{I.D.} \times 20 \mathrm{~mm}, 5 \mu \mathrm{m}$, Thermo Fisher Scientific). Chromatographic separation was performed on a reversed phase $\mathrm{C}_{18}$ column (Easy-column C18-A2, $75 \mu \mathrm{m}$ I.D. $\times 100 \mathrm{~mm}, 3 \mu \mathrm{m}$, Thermo Fisher Scientific) at a flow rate of $300 \mathrm{~nL} / \mathrm{min}$ with a $60 \mathrm{~min}$ gradient of $2 \%$ to $40 \%$ acetonitrile in $0.1 \%$ formic acid. The electrospray voltage was maintained at $2.2 \mathrm{kV}$, and the capillary temperature was set at $320^{\circ} \mathrm{C}$. The LTQ-Orbitrap was operated in data-dependent mode to simultaneously measure full scan MS spectra (m/z 300-1800) in the Orbitrap with a mass resolution of 60,000 at $\mathrm{m} / \mathrm{z} 400$. After full-scan survey, 15 most abundant ions as detected in the full-MS scan and were measured in the LTQ-Orbitrap using collision-induced dissociation (CID). Protein identification was performed with Proteome Discoverer 1.4 software (Thermo). MS/MS data were submitted to the UniProt human protein database using SequestHT to follow trypsin cleavage, fixed modification of carbamidomethylation of cysteine; variable modifications of methionine oxidation, a maximum of two missed cleavages, and false discovery rate calculated by searching the decoy database. Other parameters 
were set as default. The results were imported into Microsoft excel for further analysis.

Pull-down/immunoblotting for target validation. For putative target identification from above in vitro labeling experiments with $\mathbf{2 4}$, H9 cells lysates were treated with the probe 24, followed by click chemistry with Biotin- $\mathrm{N}_{3}$ and pulled-down with streptavidin magnetic beads. The lysate was then separated by SDS-PAGE, transferred onto polyvinylidene fluoride membrane and analyzed by Western blotting with antibodies specific for the native form of proteins (PRM2, TUBA1C, CCT7, TCP1, RUVBL1, DARS and ATP5B). Control groups were treated with DMSO instead of the probe 24 .

Surface plasmon resonance (SPR) experiments. SPR experiments were performed by Biacore $8 \mathrm{~K}$ (GE Health) to evaluate the associating affinities of baculiferins with RUVBL1 and DARS respectively. The recombinant RUVBL1-His (Proteintech, cat: Ag0281) and DARS-His (Prospec, cat: enz-591) fusion proteins were immobilized on a sensor chip (CM5). Gradient doses of each compound were subsequently injected as analytes. PBS-P (PBS buffer with 5\% DMSO and 0.5\% tween-20) was used as the running buffer. The data were analyzed by Biacore Insight Evaluation Software 1.0.5.11069.

Cells and plasmids. HEK293T cells were cultured in DMEM (GBICO) supplemented with 10\% fetal bovine serum (FBS) (GBICO). SupT1 cells were maintained in RPMI-1640 (GBICO) containing 10\% FBS. Transfection of HEK293T was performed using Lipofectamine 2000 (Invitrogen) and Lipofectamine RNAi MAX (Invitrogen) respectively according to the manufacturer's manuals. Human cDNAs containing completely coding regions for DARS were cloned into the pcDNA3.1 vector (Invitrogen) using BamHI and XhoI to express with a fused flag-tag at the C-terminus. SiRNAs were purchased from RIBO Biotechnology Inc with targeting gene ID 8607 and 1615 respectively.

Western blots. Western blots were probed with monoclonal antibodies that are specifically reacted with DARS (Abcam, ab206695) and $\beta$-actin (Abcam, ab8224). Detection of proteins was performed by enhanced chemiluminescence (Millipore) 
using secondary antibodies anti-mouse and anti-rabbit (ZSGB Biotechnology Inc). Bands in western blots were quantitated using ChemiDoc (Bio-Rad) automated digitizing system.

HIV-1 Infectivity assay. HEK293T cells were co-transfected with $300 \mathrm{ng}$ pNL4-3Luc(R-E-), 200 ng VSVG and Si-DARS/DARS (0, 100, 200, 500ng) plasmid DNA in 6-well-plates for $12 \mathrm{~h}$. The supernatant was collected and filtered by a 0.45 $\mu \mathrm{m}$ filter, and then used to infect the SupT1 cells $(1 \times 105)$ in 96 -well plates. After $48 \mathrm{~h}$, SupT1 cells were lysed and firefly luciferase activities were determined by a firefly Luciferase Assay System (Promega). The DMSO used to dissolve compound was diluted into $1 \%(\mathrm{v} / \mathrm{v})$. P24 in the supernatant was measured using a p24-ELISA assay system according to the manufacturer's manual.

Viral RNA isolation and quantification. HEK293T cells were transfected with SiRNA targeting RUVBL1 and DARS respectively. After $48 \mathrm{~h}$, total RNA was isolated using TRIzol (Invitrogen). The RNAs were analyzed by real-time PCR using the KAPA SYBR FAST qPCR Kit (TAKARA) and the Mx3000PTM Quantitative PCR System thermal cycler (Strata gene). The primers used in real-time PCR involved qDARS-forward 5'-AACAGTGCTTCTTAGTCCTA-3', qDARS-reverse 5'-CTGAACATGTAACTCAACGT-3', qRUVB1-forward 5'-ACCATTAGCCATGTGATCAT-3', qRUVB1-reverse 5 TCTTCAGCTTCAAGGTCGAA-3';

GAPDH-forward: 5'-GAAGGTGAAGGTCGGAGT-3'; GAPDH-reverse 5'-GAAGATGGTGATGGGATTTC-3'. QRT-PCR result was normalized using GAPDH amplification levels and was calculated by $2^{-\triangle \Delta \mathrm{Ct}}$.

Molecular docking. MOE-dock was used for the molecular docking simulation of the ligand to predict the binding affinity with target protein. 2D structure of the ligand was drawn using ChemBioDraw-2014 and was converted to 3D in MOE through energy minimization. Crystal structure (PDB 4J15) was downloaded from RCSB Protein Data Bank (https://www.rcsb.org). Prior to docking, the force field of AMBER10: EHT and the implicit solvation model of Reaction Field (R-field) were selected. The receptor structures were prepared by MOE Structure Preparation and 
were protonated by module Protonate 3D. Three putative binding sites were predicted by module Site Finder, another anticodon binding site and catalytic binding site were determined by module Align/Superpose with crystal structure of PDB 1ASY to form a complex with tRNA. The docking workflow followed the "induced fit" protocol, in which the side chains of the receptor pocket could move according to ligand conformations with a constraint on their positions. The weight used for tethering side chain atoms to their original positions was 10. All docked poses of molecules were ranked by London $\mathrm{dG}$ scoring firstly, then a force field refinement was carried out on the top 30 poses followed by a rescoring of GBVI/WSA dG. The figure presenting docking results was prepared with PyMOL (DeLano Scientific LLC, CA). 


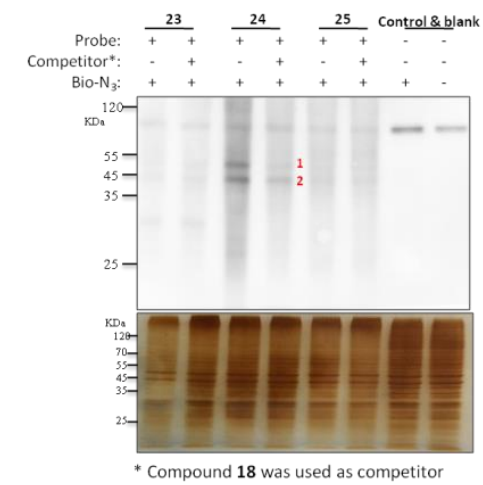

Figure S1: Probe-photolabeled HIV-1 infected H9 cell lysates separated by SDS-PAGE, transferred onto PVDF membrane and visualized by streptavidin-HRP
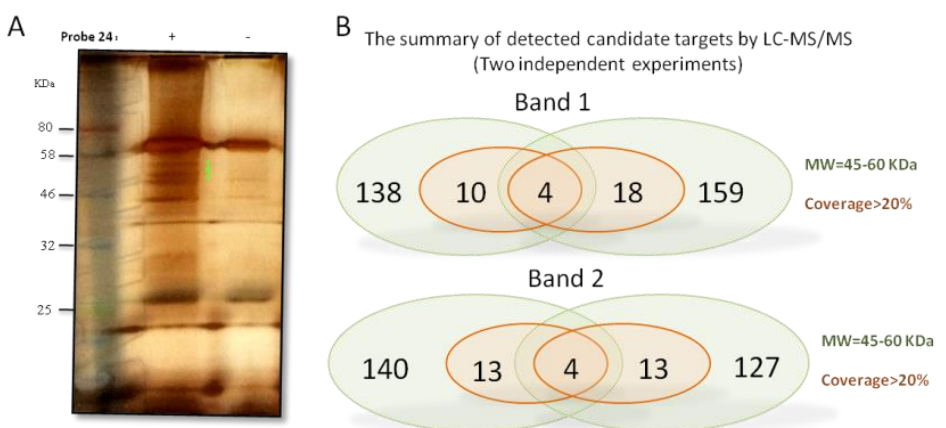

Figure S2: Photo-labeled protein enrichment and identification by LC-MS/MS

A) After the photoaffinity labeling, the biotinylated protein was enriched by streptavidin magnetic beads, which were separated by SDS-PAGE and visualized by silver stain; B) Summary of LC-MS/MS results.

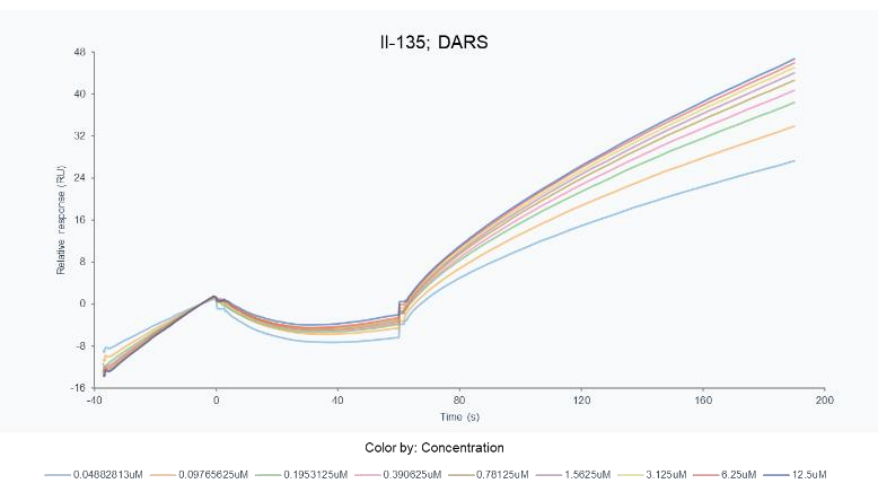

Figure S3. SPR results of II-135 to interact with DARS.

SPR experiments were performed on a Biacore 8K (GE Health) for evaluating the associating affinities of II-135 with DARS. The DARS-His (Prospec, cat: enz-591) fusion protein was immobilized on a sensor chip (CM5). Various concentrations of the compound were subsequently injected as analytes. PBS-P (PBS buffer with 5\% DMSO and 0.5\% tween-20) was used as the running buffer. For all binding studies, the analytes were added to the running buffer at a flow rate of $30 \mu \mathrm{l} / \mathrm{min}$, a contact time of $60 \mathrm{~s}$, and a dissociation time of $60 \mathrm{~s}$. The data was analyzed by Biacore Insight Evaluation Software 1.0.5.11069. 

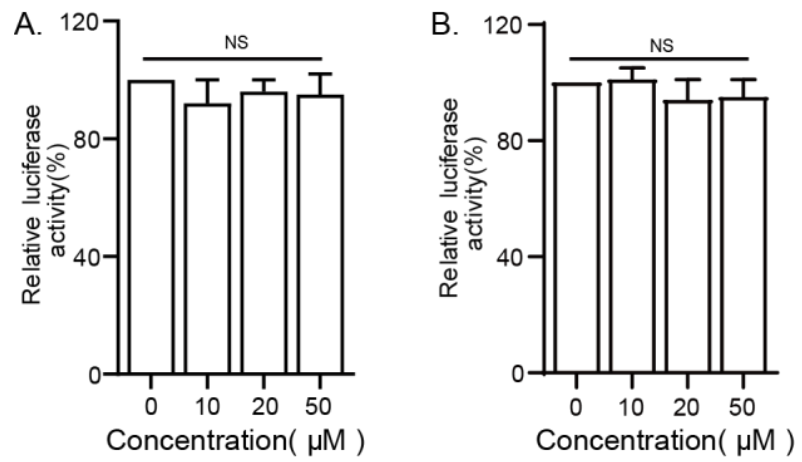

Figure S4. Effects of 18 (A) and 24 (B) on luciferase activity. 

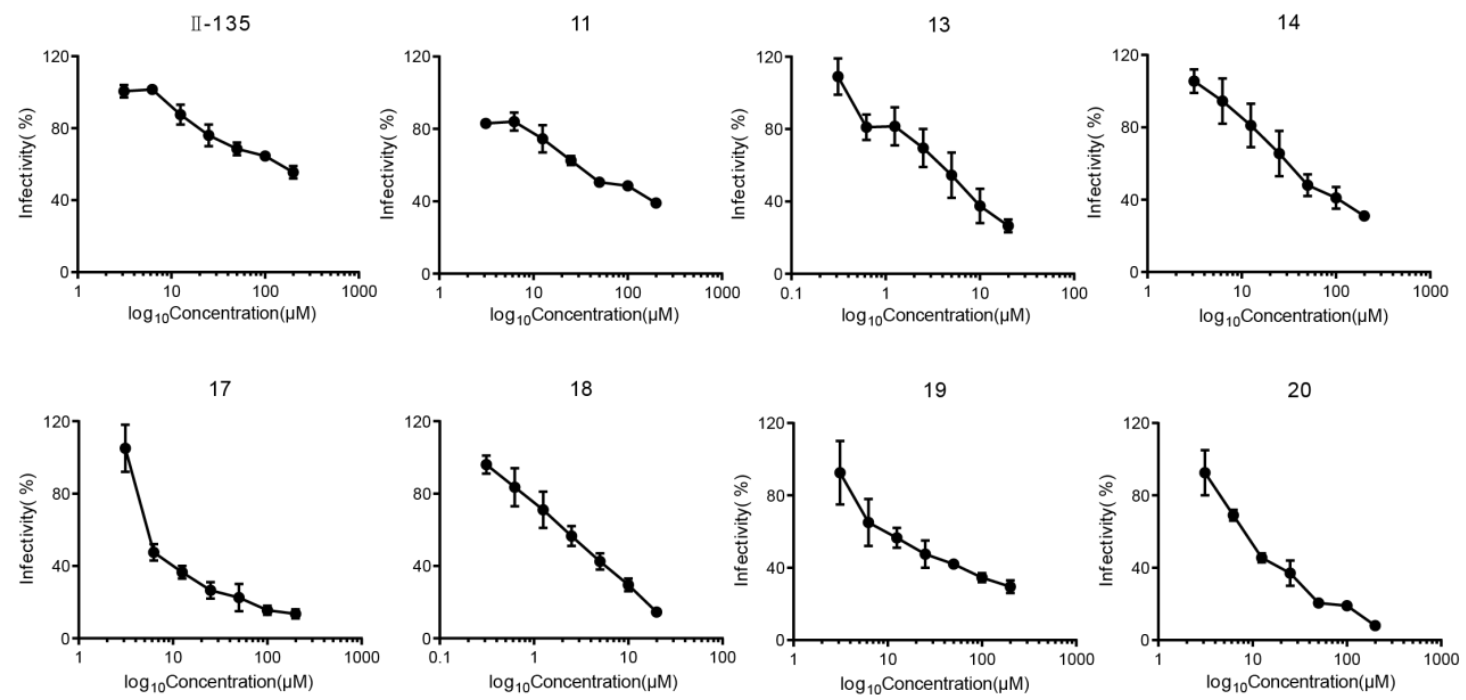

Figure S5. Inhibitory effects of synthesized compounds toward VSV-G pseudotyped HIV-1 in infected SupT1 cells
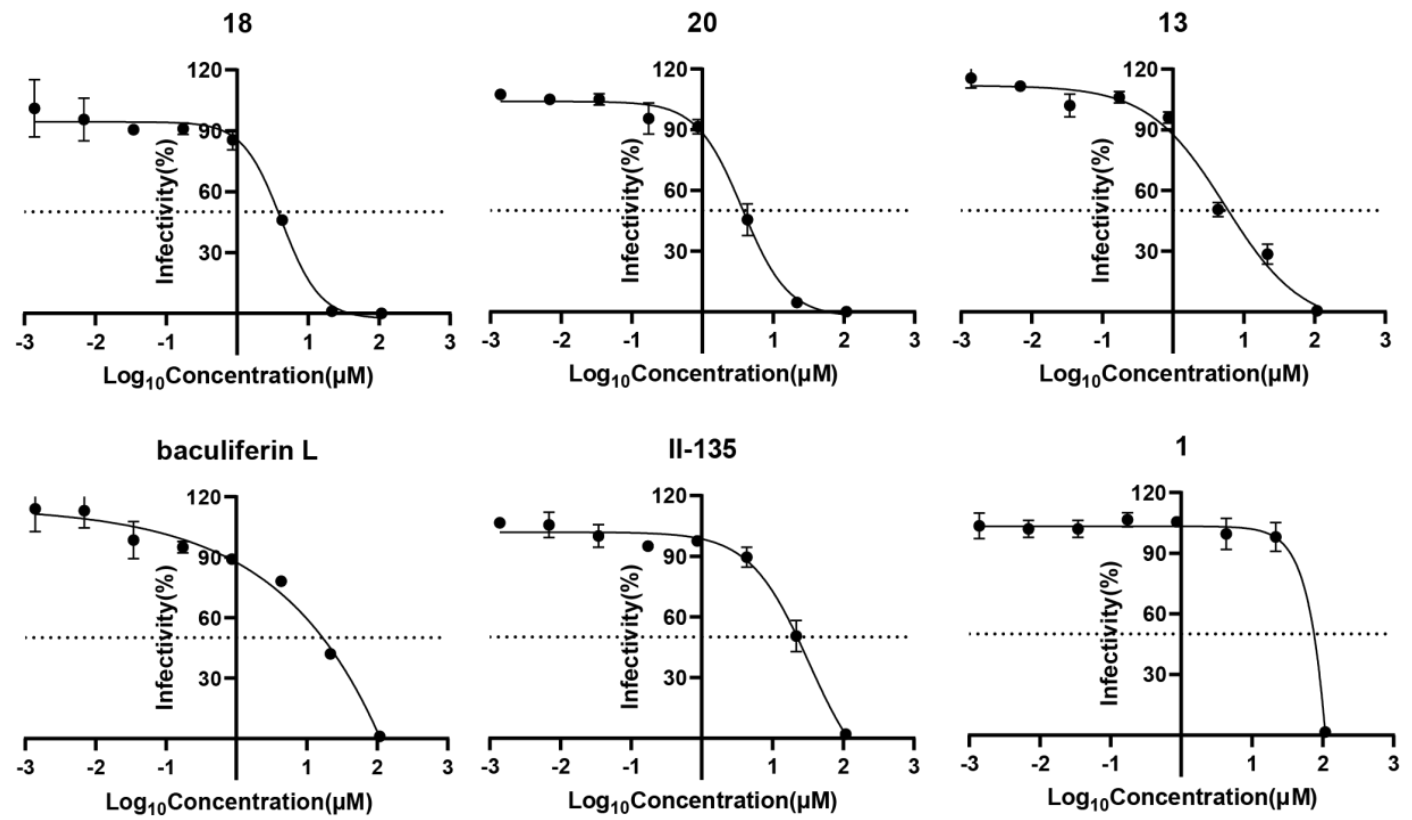

Figure S6. Inhibitory effects of compounds toward HIV-1 strain SF33 in infected TZM-bl cells 
Table S1 Ligand Interaction Report of Binding Pose for $\mathbf{1 3}$

\begin{tabular}{llllrr}
\hline Ligand & & Receptor & Interaction & Distance $(\AA)$ & E (kcal/mol) \\
\hline C44 & OE2 & GLU424 & H-donor & 3.49 & -0.2 \\
O34 & NH1 & ARG431 & H-acceptor & 2.88 & -5.2 \\
O46 & N & SER427 & H-acceptor & 3.31 & -1.4 \\
6-ring & NE2 & GLN251 & pi-H & 4.03 & -0.6 \\
6-ring & CB & GLN251 & pi-H & 4.4 & -0.5 \\
6-ring & CG & PRO400 & pi-H & 4.54 & -0.3 \\
6-ring & CG & PRO400 & pi-H & 3.74 & -0.4 \\
5-ring & CB & SER427 & pi-H & 3.56 & -1 \\
\hline
\end{tabular}

Table S2 Ligand Interactions Report of Binding Pose of $\mathbf{1 8}$

\begin{tabular}{clllrr}
\hline Ligand & Receptor & Interaction & Distance & E (kcal/mol) \\
\hline C & OE1 GLU424 & H-donor & 3.62 & -0.2 \\
O & N & SER427 & H-acceptor & 3.03 & -1.8 \\
6-ring & CG & PRO400 & pi-H & 3.77 & -0.2 \\
6-ring & CB & SER427 & pi-H & 4.21 & -0.2 \\
5-ring & CB & SER427 & pi-H & 3.73 & -1.1 \\
\hline
\end{tabular}




\section{References}

1. Sterling, J., et al., Novel dual inhibitors of AChE and MAO derived from hydroxy aminoindan and phenethylamine as potential treatment for alzheimer's disease. J. Med. Chem. 2002, 45 (24), 5260-5279.

2. He, F., et al., High performance polymer derived from a biorenewable plant oil (anethole). ACS Sustain. Chem. Engin. 2017, 5(3), 2578-2584.

3. Beugelmans, R., et al., Synthetic studies towards western and eastern macropolypeptide subunits of kistamycin. Tetrahedron 1999, 55(16), 5089-5112.

4. Tian, G., P. Fedoseev and E.V. Van der Eycken, Hypervalent iodine(III)-mediated cascade cyclization of propargylguanidines and total syntheses of kealiinine B and C. Chemistry 2017, 23(22), 5224-5227.

5. Caldwell, C.G., et al., Preparation of diphenyloxaspiroalkanes as tachykinin receptor antagonists. 1998, Merck \& Co., Inc., USA; Caldwell, Charles G.; Chiang, Yuan-Ching; Dorn, Conrad; Finke, Paul; Hale, Jeffrey; Maccoss, Malcolm; Mills, Sander; Robichaud, Albert . p. 303 pp.

6. Ohta, T., et al., Design and synthesis of lamellarin D analogues targeting topoisomerase I. J. Org. Chem. 2009, 74(21), 8143-8153.

7. Maurin, C., F. Bailly, P. Cotelle, Facile access to methoxylated 2-phenylnaphthalenes and epoxydibenzocyclooctenes. Tetrahedron 2005, 61, 7054-7058.

8. Gérard-Hirne, T., et al., Photoactivatable oligonucleotide probes to trap single-stranded DNA binding proteins: updating the potential of 4-thiothymidine from a comparative study. Biochimie 2018, 154, 164-175. 


\section{Annex}

$\begin{array}{lll}{ }^{1} \mathrm{H} \mathrm{NMR}: \mathrm{B}-1 \mathrm{a} & \overline{0} & 0 \\ \text { Solvent: } \mathrm{CDCl}_{3} & 0\end{array}$<smiles>COc1ccc(C(=O)C(=O)O)cc1OC</smiles>

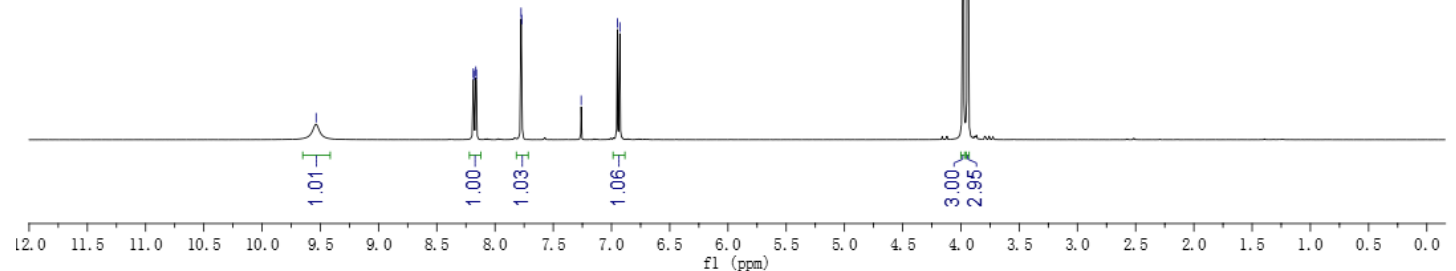

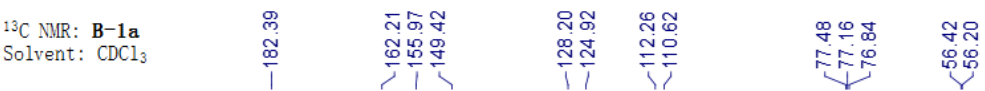<smiles>COc1ccc(C(=O)C(=O)O)cc1OC</smiles>

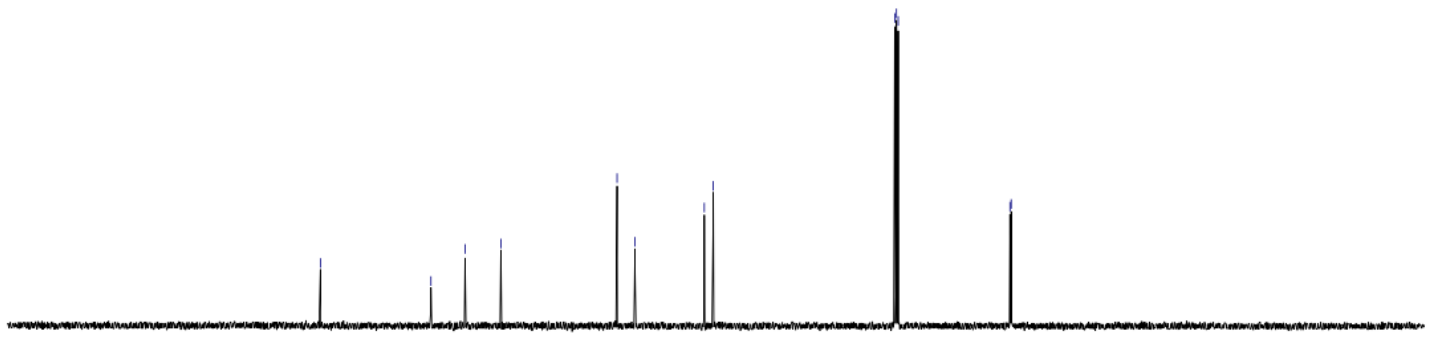

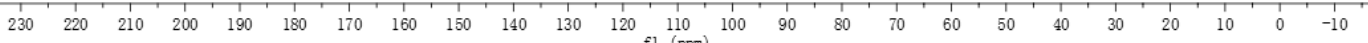




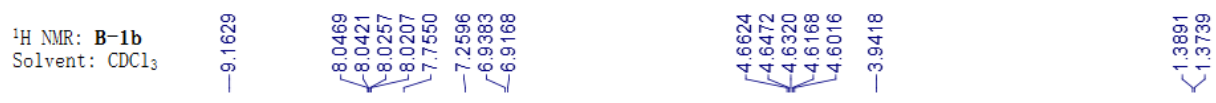<smiles>COc1ccc(C(=O)C(=O)O)cc1OC(C)C</smiles>

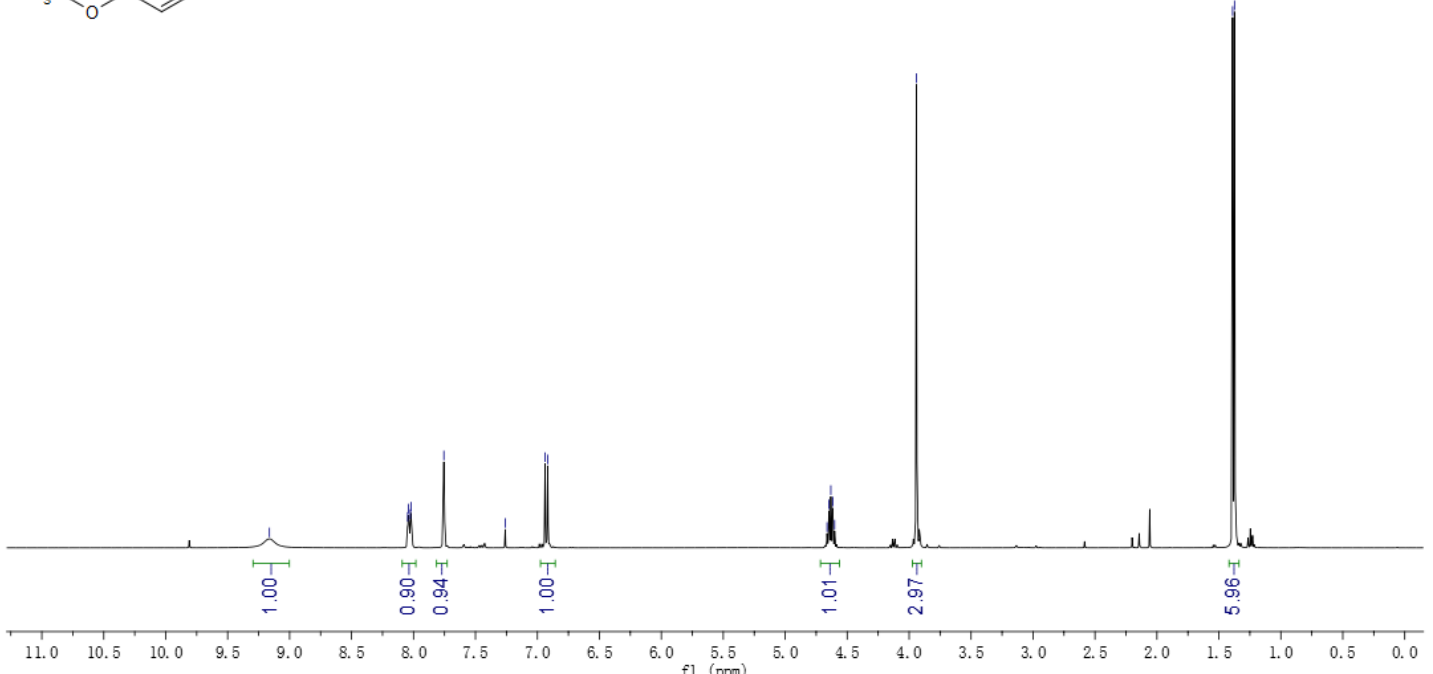

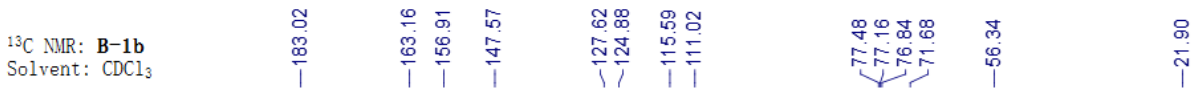<smiles>COc1ccc(C(=O)C(=O)O)cc1OC(C)C</smiles>

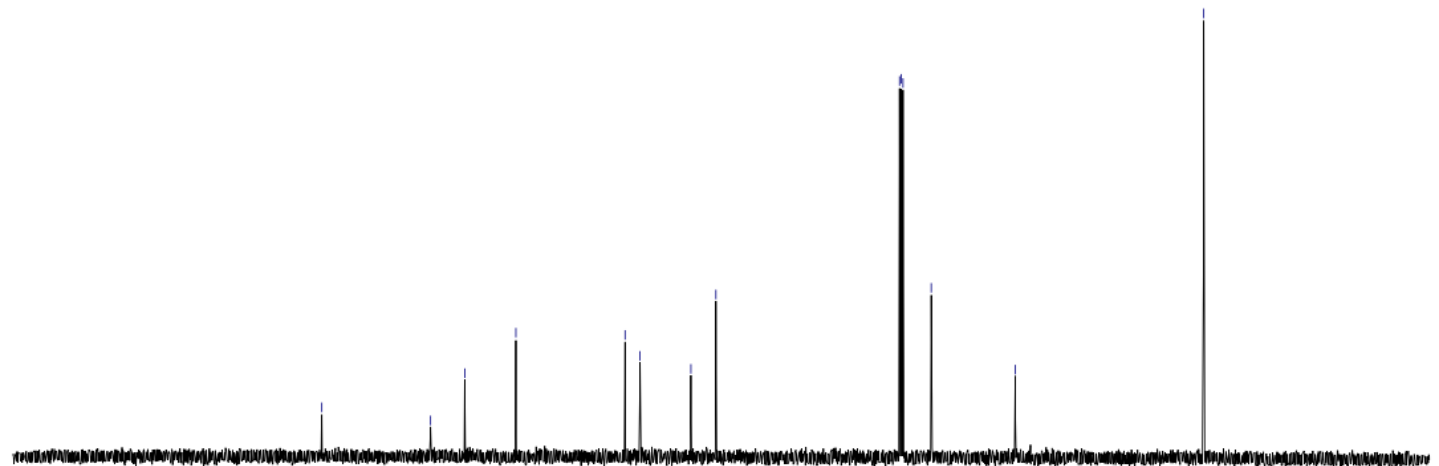

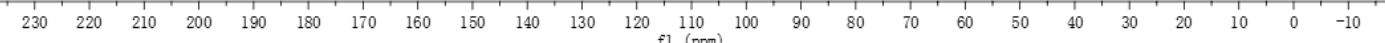


${ }^{1} \mathrm{H}$ NMR: $\mathrm{B}-2 \mathrm{a}$
Solvent: $\mathrm{CDCl}$

(1)

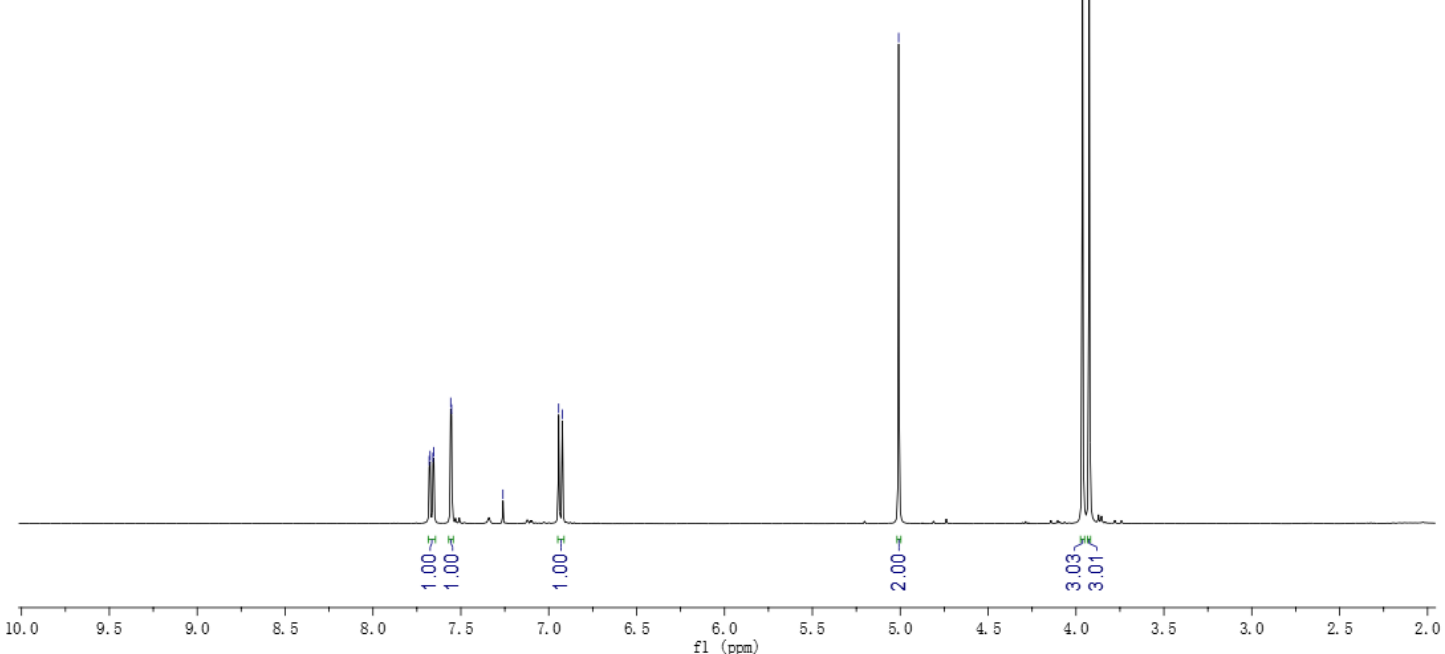

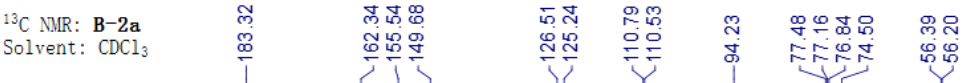<smiles>COc1ccc(C(=O)C(=O)OCC(Cl)(Cl)Cl)cc1OC</smiles>

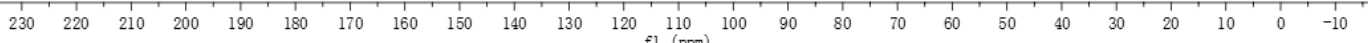


<smiles>COc1ccc(C(=O)C(=O)OCC(C)(Cl)Cl)cc1OC(C)C</smiles>

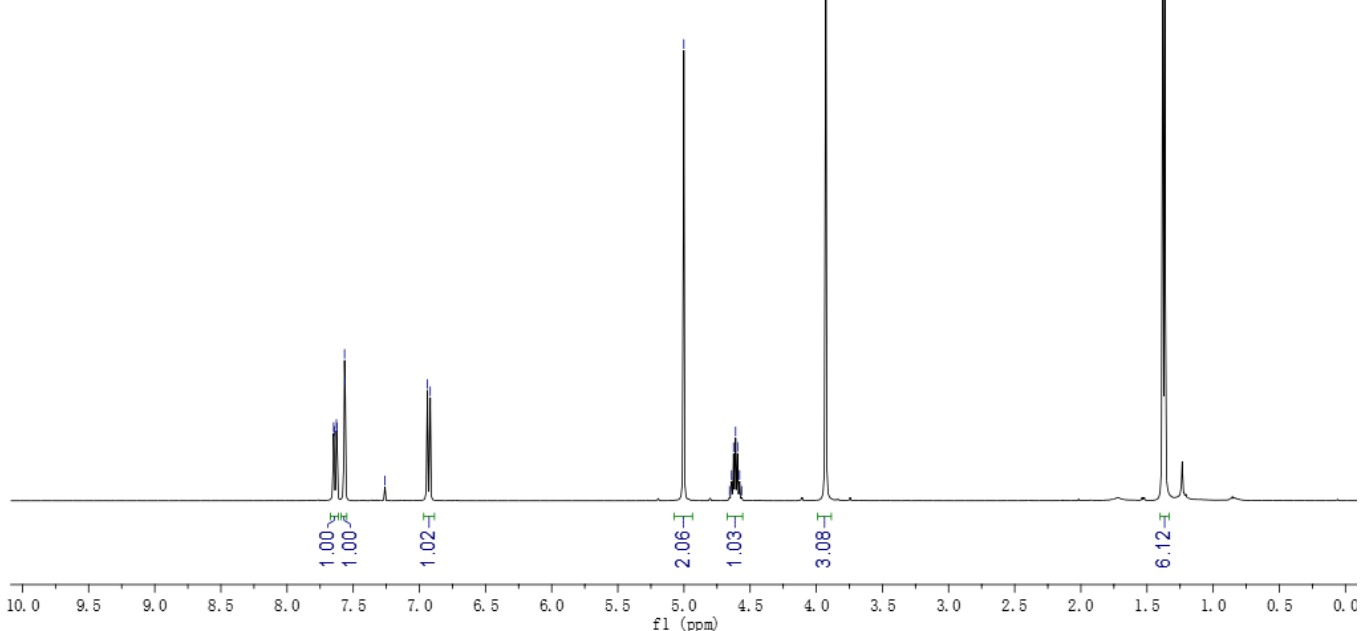

${ }^{13} \mathrm{C}$ NMR: B-2

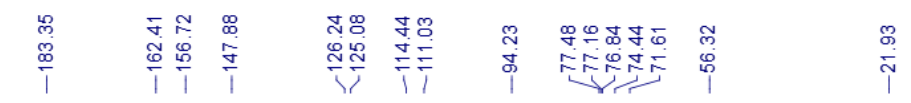<smiles>COc1ccc(C(=O)C(=O)OCC(Cl)(Cl)Cl)cc1OC(C)C</smiles>

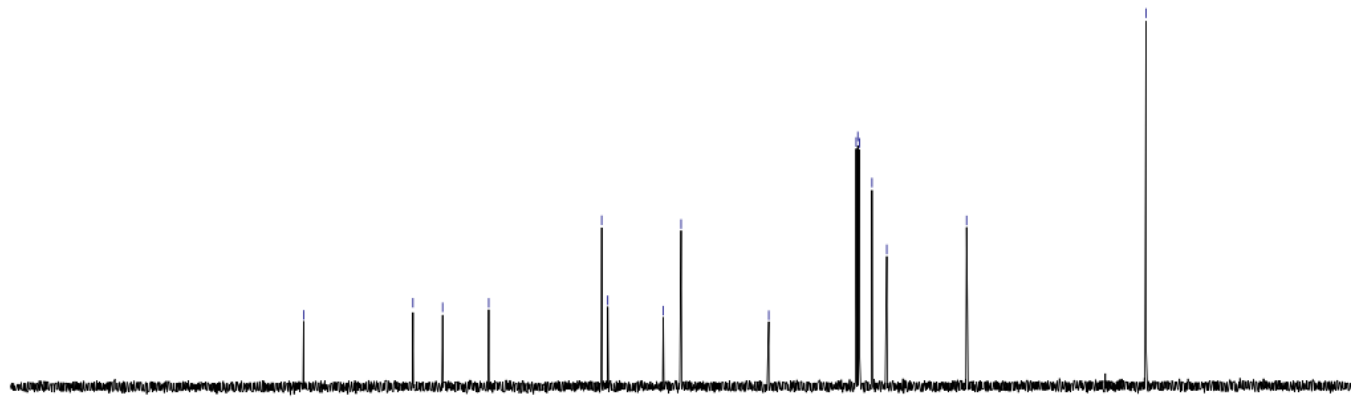

$\begin{array}{llllllllllllllllllllllllllllllllllllllllll}230 & 220 & 210 & 200 & 190 & 180 & 170 & 160 & 150 & 140 & 130 & 120 & 110 & 100 & 90 & 80 & 70 & 60 & 50 & 40 & 30 & 20 & 10 & 0 & -10\end{array}$ 


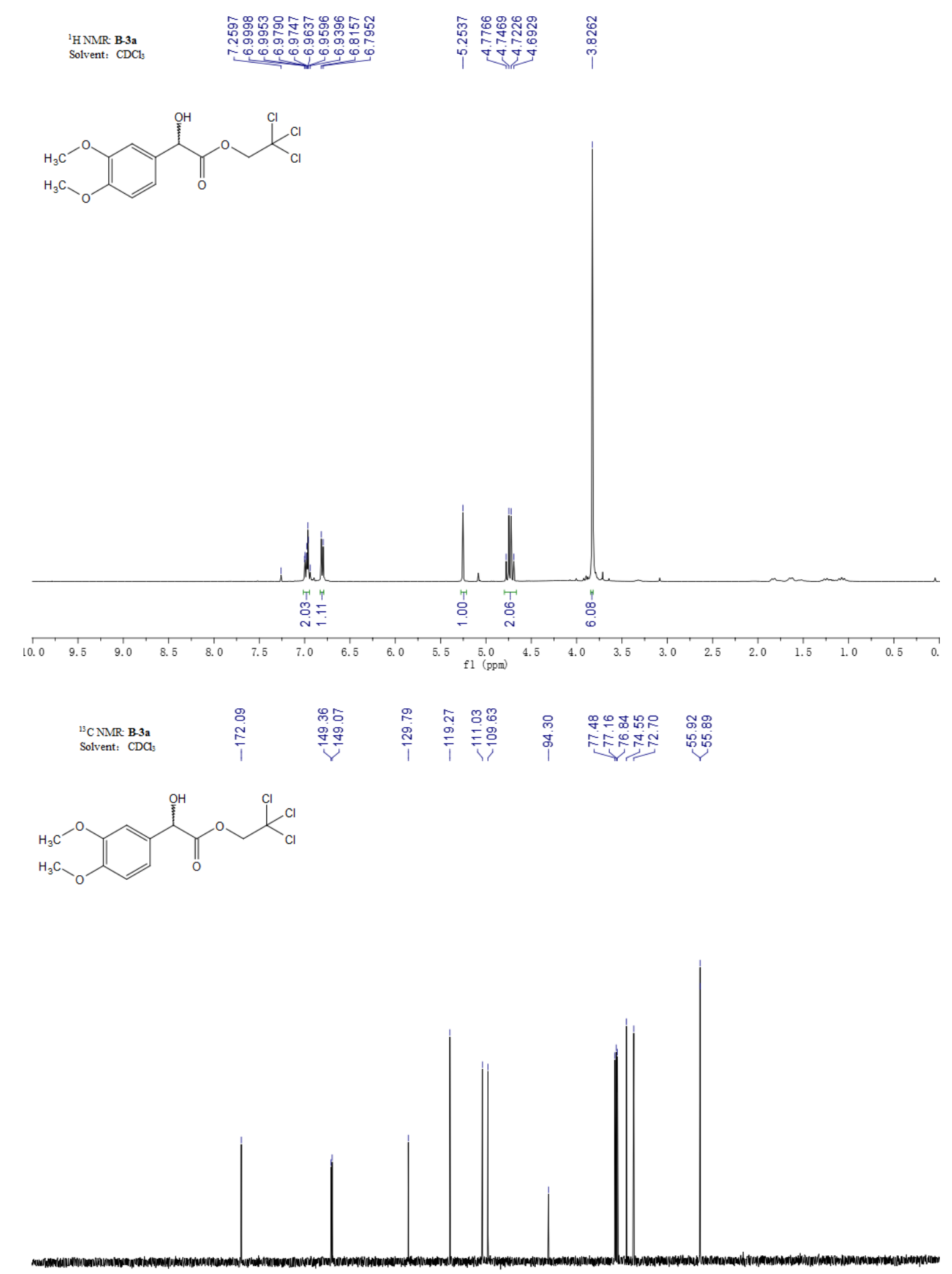

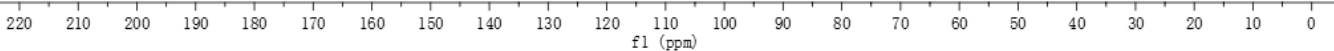


IH NMR: $\mathbf{B}-3 \mathbf{b}$
Solvent: $\mathrm{CDCl}$

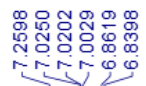

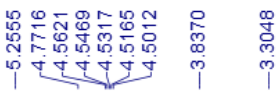

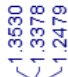<smiles>COc1ccc([C@@H](O)C(=O)OCC(Cl)(Cl)Cl)cc1OC(C)C</smiles>

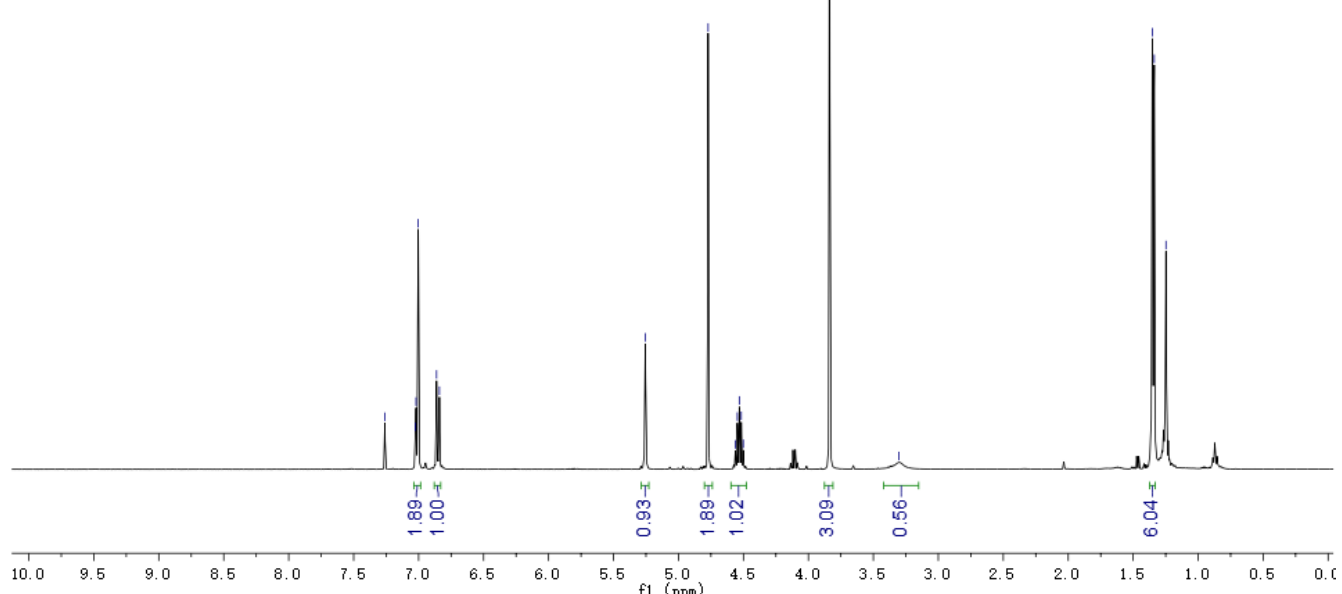

${ }^{13} \mathrm{C}$ NMR: B-3b

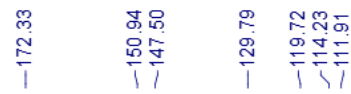

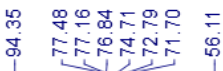

$\stackrel{\infty}{\underset{1}{1}}$<smiles>COc1ccc([C@@H](O)C(=O)OCC(Cl)(Cl)Cl)cc1OC(C)C</smiles>

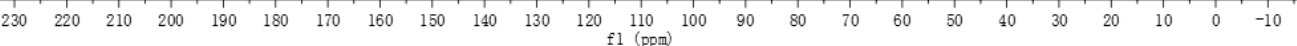




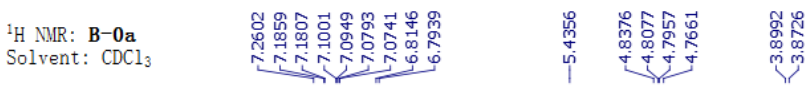
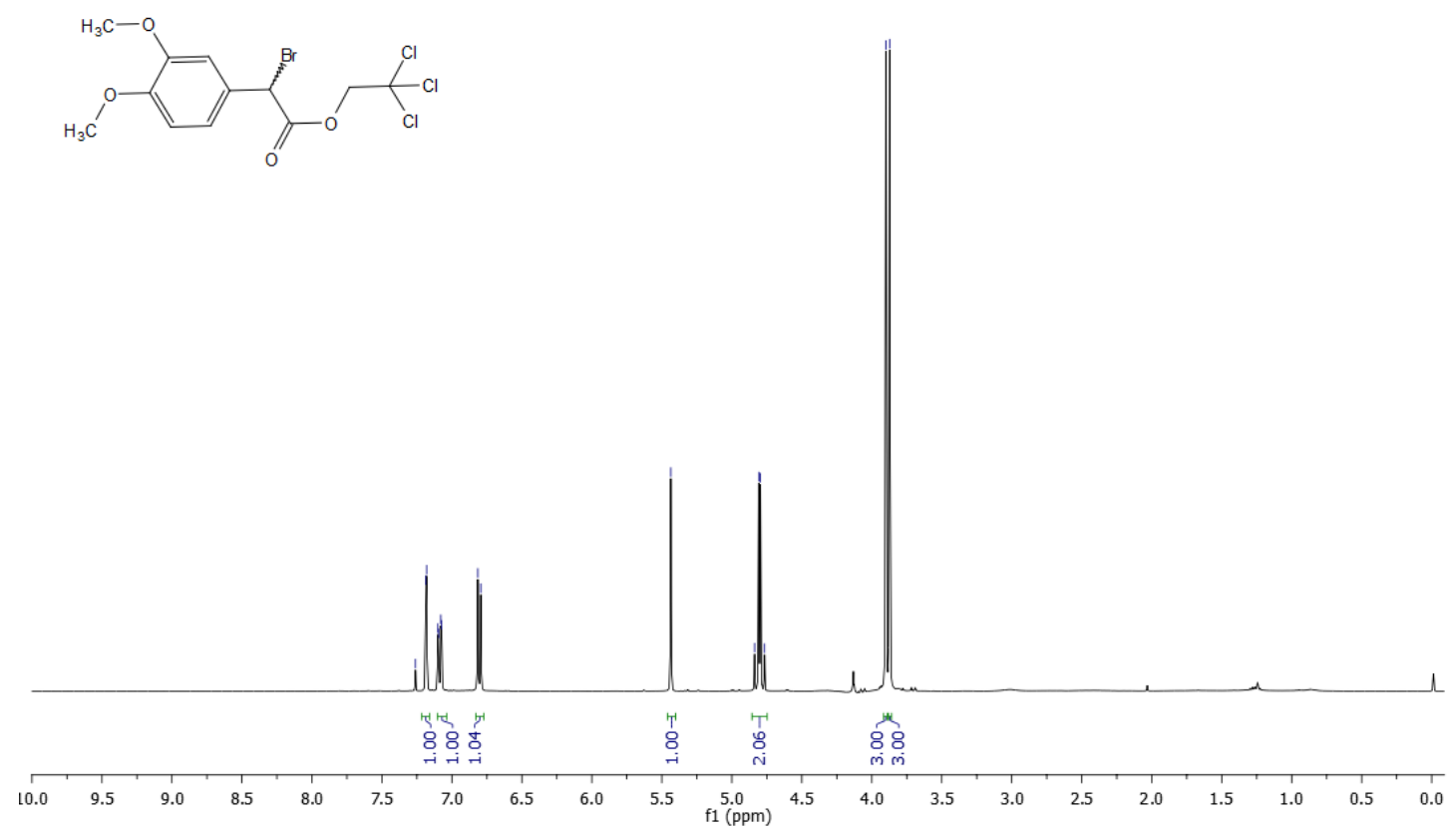

${ }^{13} \mathrm{C}$ NMR: B-0a

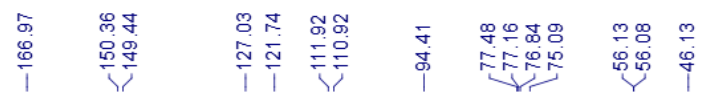
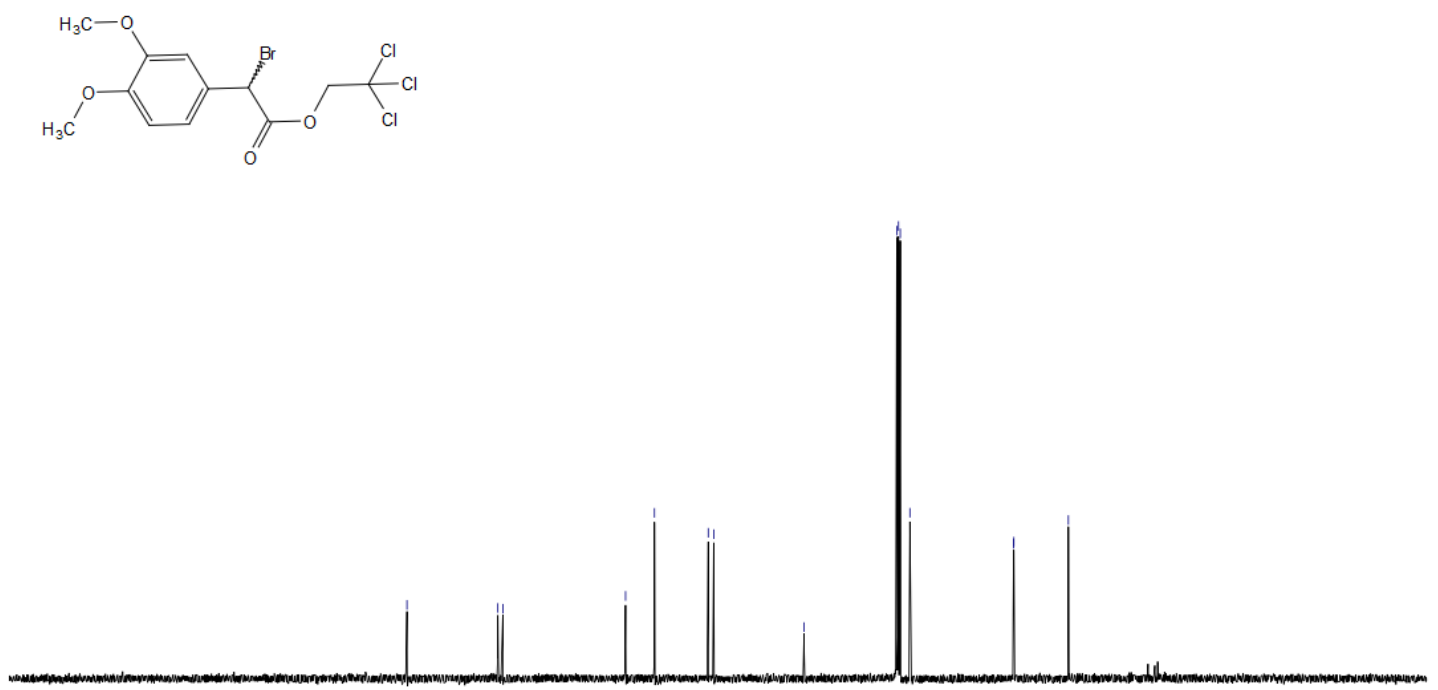

$\begin{array}{llllllllllllllllllllllllllllllllll}230 & 220 & 210 & 200 & 190 & 180 & 170 & 160 & 150 & 140 & 130 & 120 & 110 & 100 & 90 & 80 & 70 & 60 & 50 & 40 & 30 & 20 & 10 & 0 & -10\end{array}$ 
${ }^{1} \mathrm{H}$ NUR: B- 0

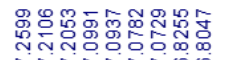

nivingonge

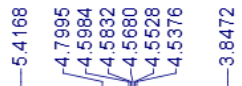

裉

(l)

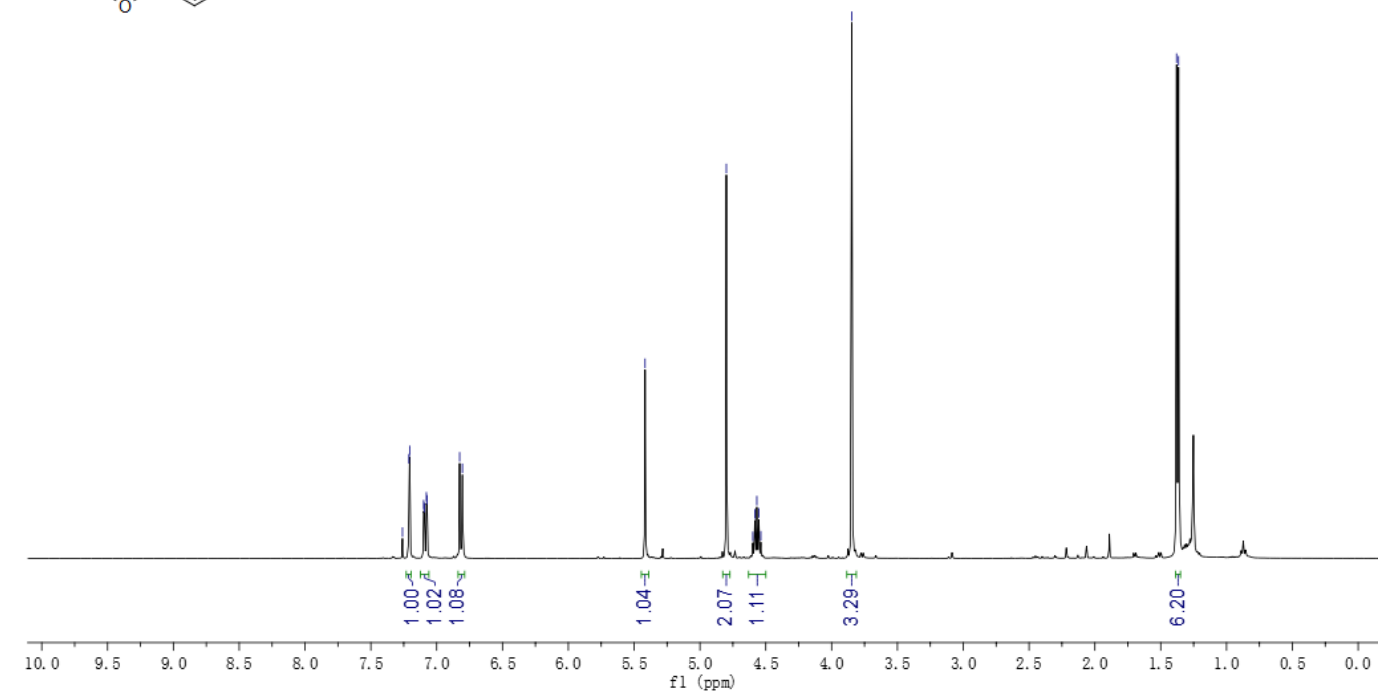

${ }^{13} \mathrm{C}$ NMR: B- $-0 \mathrm{~b}$

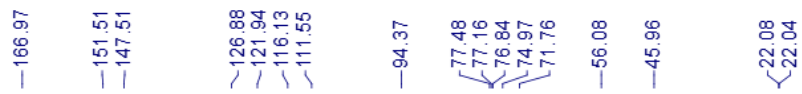<smiles>COc1ccc(C(Br)C(=O)OCC(Cl)(Cl)Cl)cc1OC(C)C</smiles>

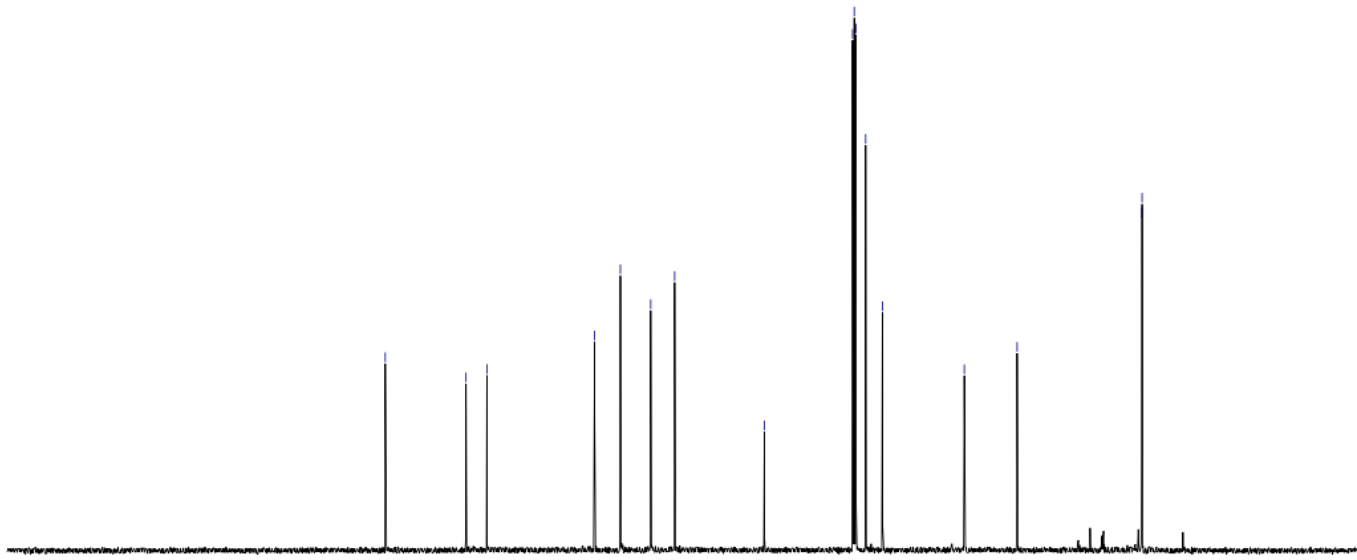

$\begin{array}{lllllllllllllllllllllllllllllll}230 & 220 & 210 & 200 & 190 & 180 & 170 & 160 & 150 & 140 & 130 & 120 & 110 & 100 & 90 & 80 & 70 & 60 & 50 & 40 & 30 & 20 & 10 & 0 & -10\end{array}$ 


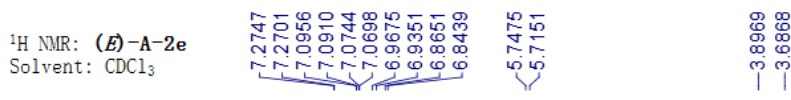<smiles>CO/C=C/c1ccc(OC)c(Cl)c1</smiles>

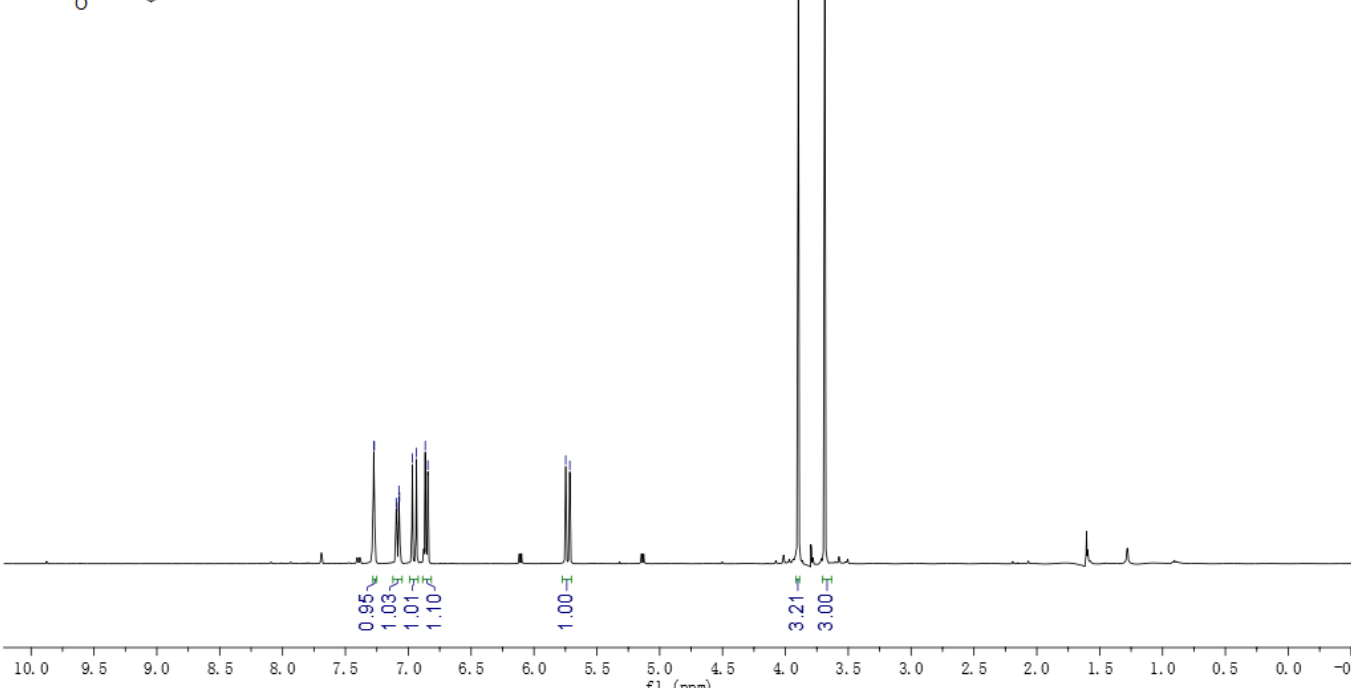

${ }^{13} \mathrm{C}$ NMR: $(E)-\mathrm{A}-2 \mathrm{e}$ Solvent: $\mathrm{CDCl}_{3}$ 更<smiles>CO/C=C/c1ccc(OC)c(Cl)c1</smiles>

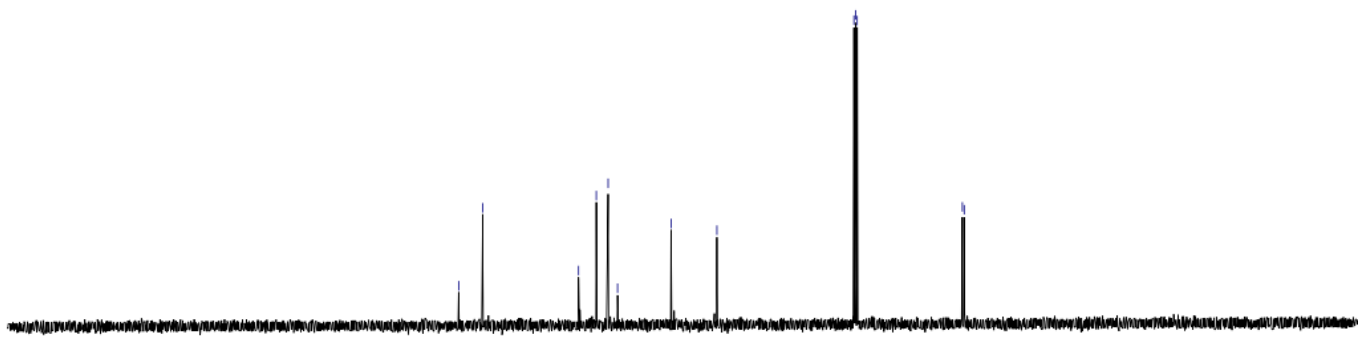

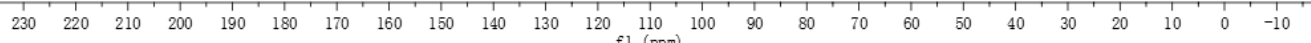




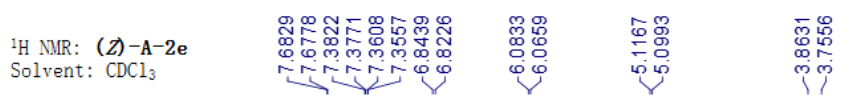<smiles>CO/C(C)=C/c1ccc(OC)c(Cl)c1</smiles>

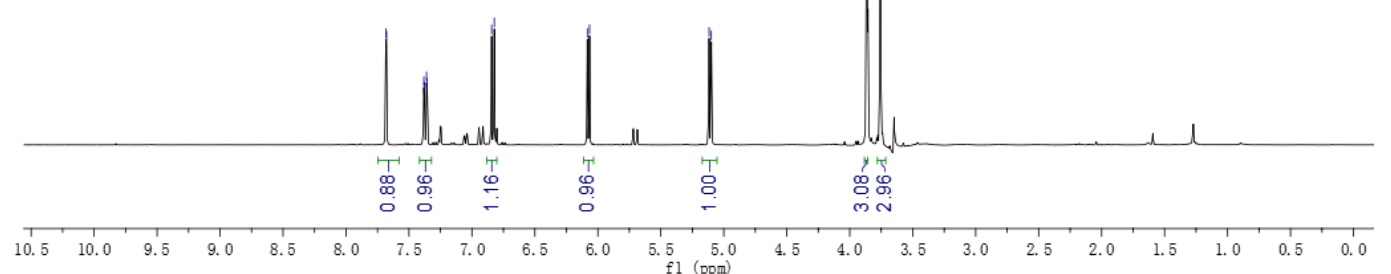

${ }^{13} \mathrm{C}$ NMR: $(Z)-\mathrm{A}-2 \mathrm{e}$ Solvent: $\mathrm{CDCl}_{3}$

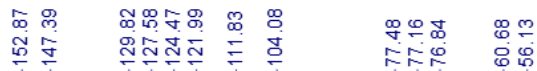<smiles>CO/C=C/c1ccc(OC)c(Cl)c1</smiles>

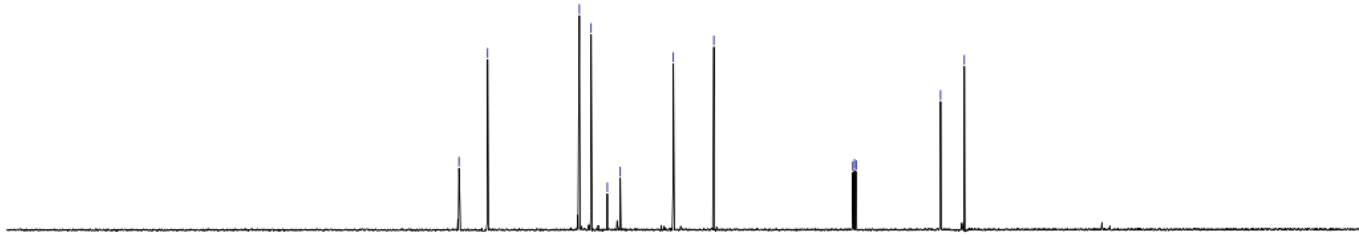

$\begin{array}{llllllllllllllllllllllllllllllllllllllllll}230 & 220 & 210 & 200 & 190 & 180 & 170 & 160 & 150 & 140 & 130 & 120 & 110 & 100 & 90 & 80 & 70 & 60 & 50 & 40 & 30 & 20 & 10 & 0 & -10\end{array}$ 


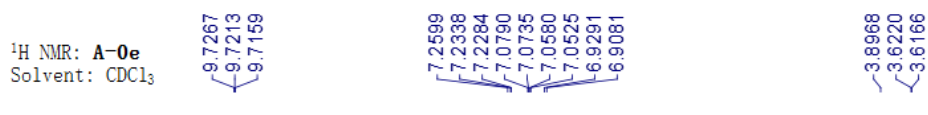<smiles>COc1ccc(CC=O)cc1Cl</smiles>

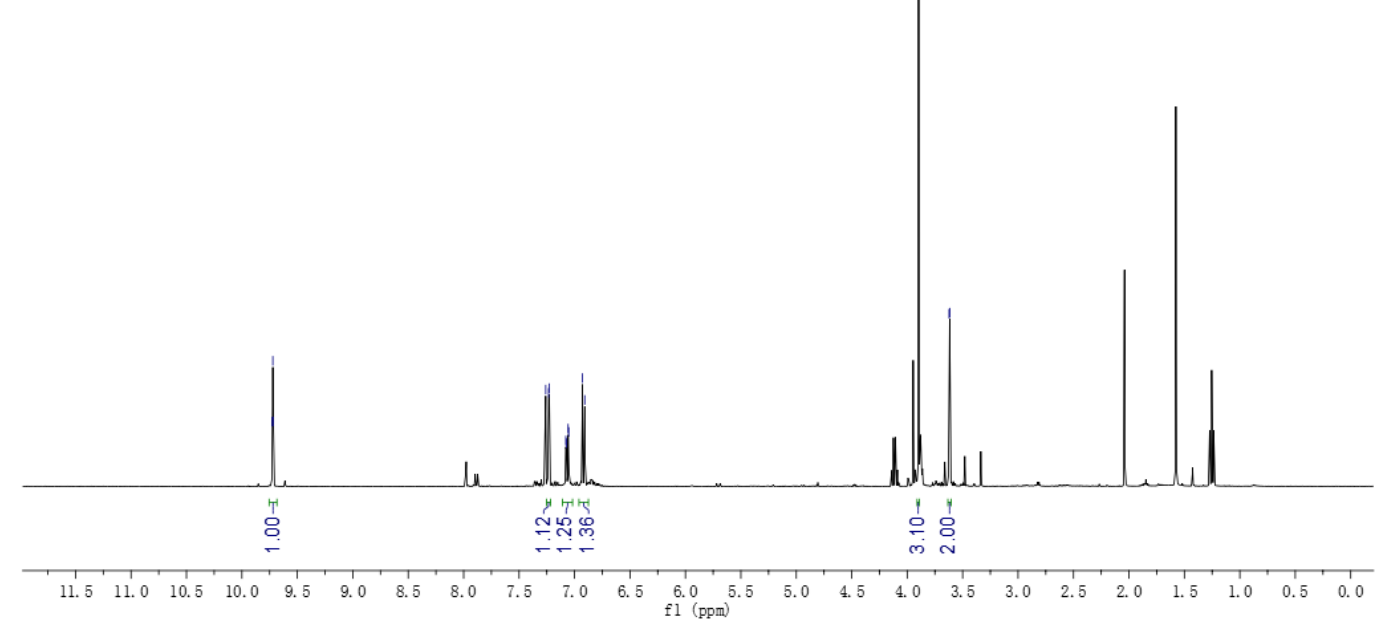

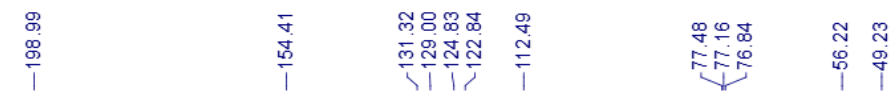

${ }^{13} \mathrm{C}$ NMR: A-0e

Solvent: $\mathrm{CDCl}_{3}$<smiles>COc1ccc(CC=O)cc1Cl</smiles>

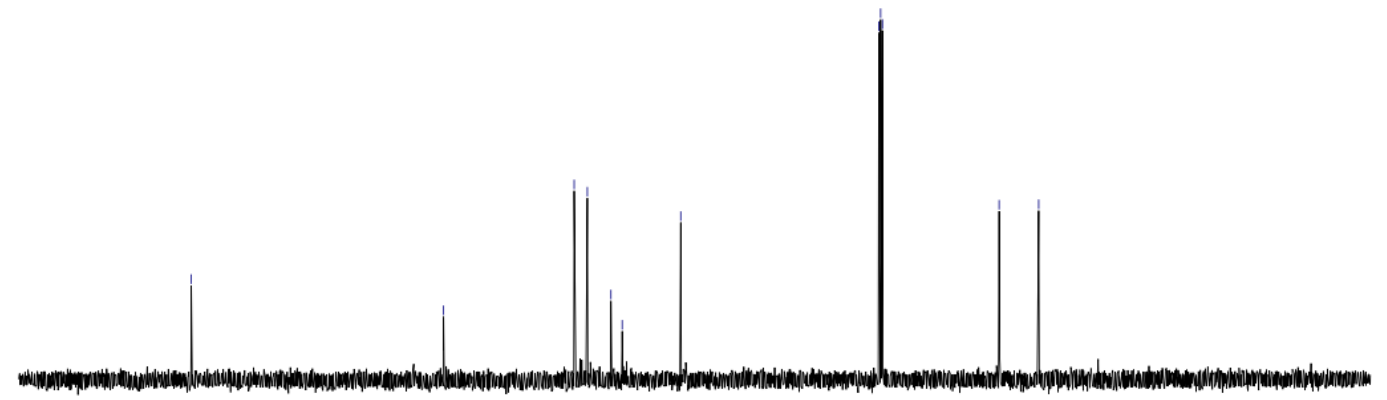

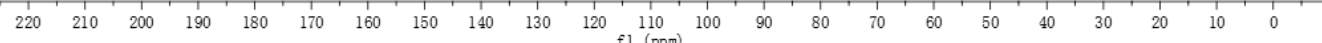


1H NMR: A-0f
Solvent: $\mathrm{CDCl}_{3}$

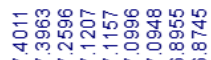

rirring

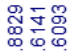

$m_{0}$

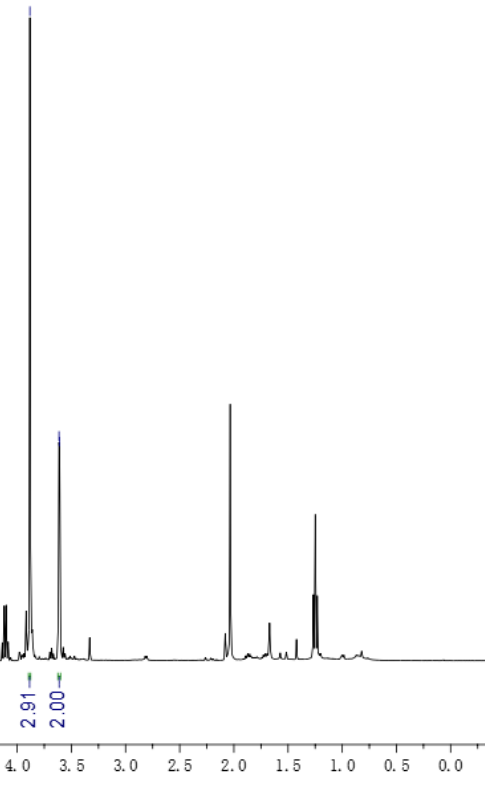

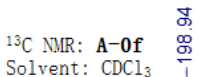

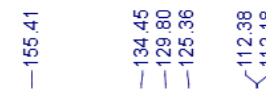

篎禹

量<smiles>COc1ccc(CC=O)cc1Br</smiles>

$\begin{array}{llllllllllllllllllllllllllllll}230 & 220 & 210 & 200 & 190 & 180 & 170 & 160 & 150 & 140 & 130 & 120 & 110 & 100 & 90 & 80 & 70 & 60 & 50 & 40 & 30 & 20 & 10 & 0 & -10\end{array}$ 

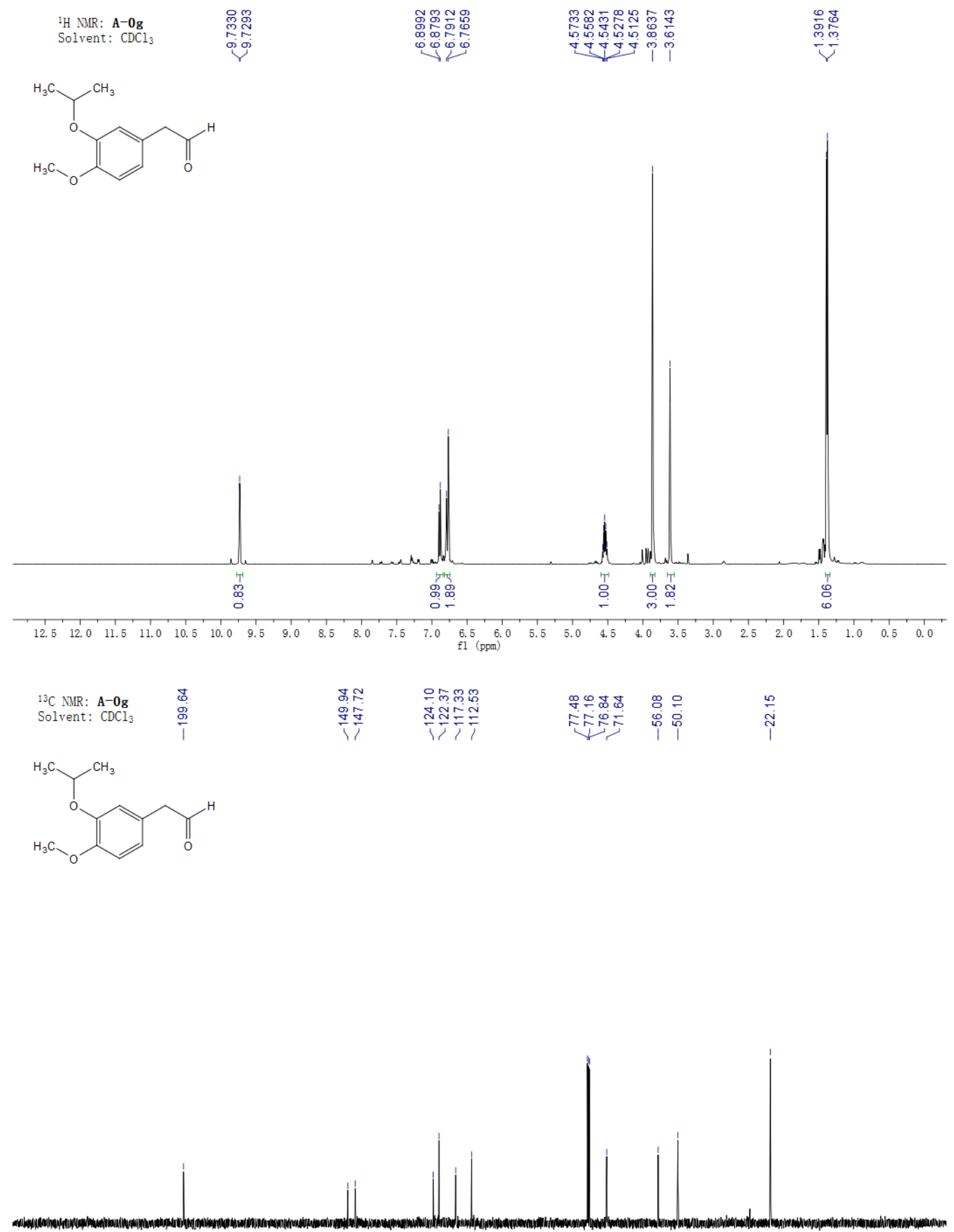

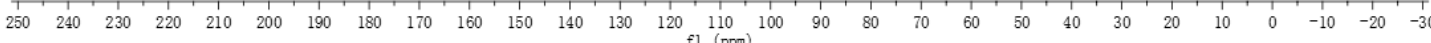


<smiles>COc1ccc(-c2ccc3c(OC(C)C)c(OC)ccc3c2)cc1OC(C)C</smiles>

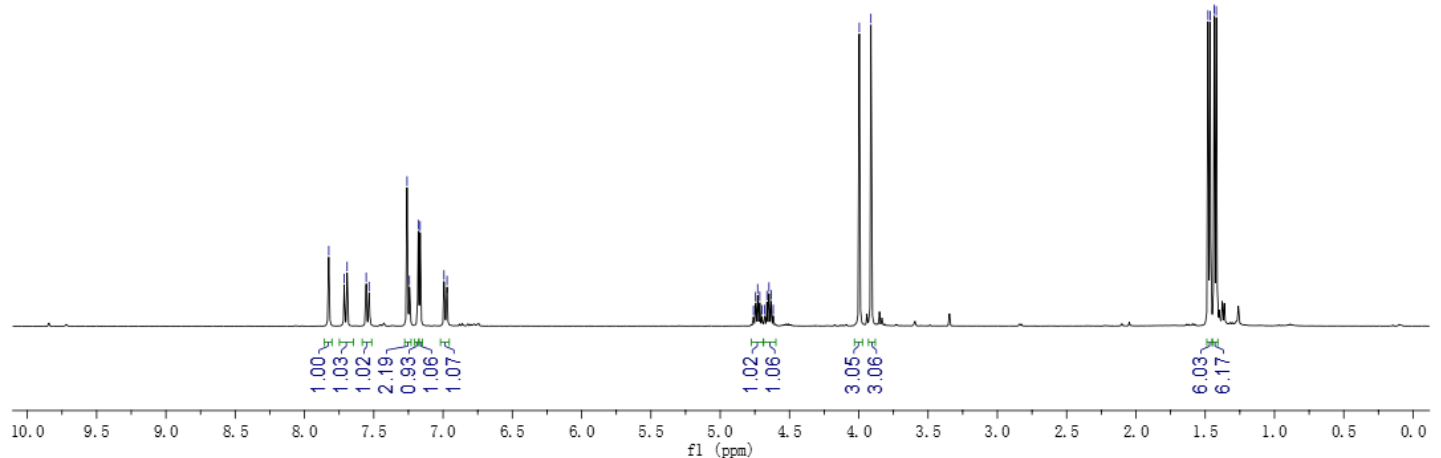

${ }^{13} \mathrm{C}$ NMR: $\mathbf{A}-\mathbf{0 g}-$ by

ดำ

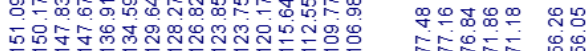<smiles>COc1ccc(-c2ccc3c(OC(C)C)c(OC)ccc3c2)cc1OC(C)C</smiles>

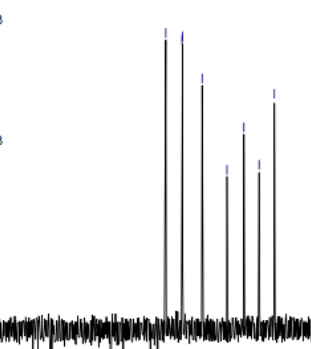


${ }^{1} \mathrm{H}$ NMR: 1

Solvent: $\mathrm{CDCl}_{3}$

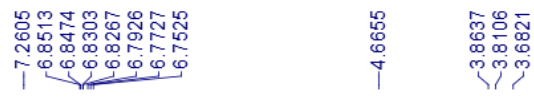
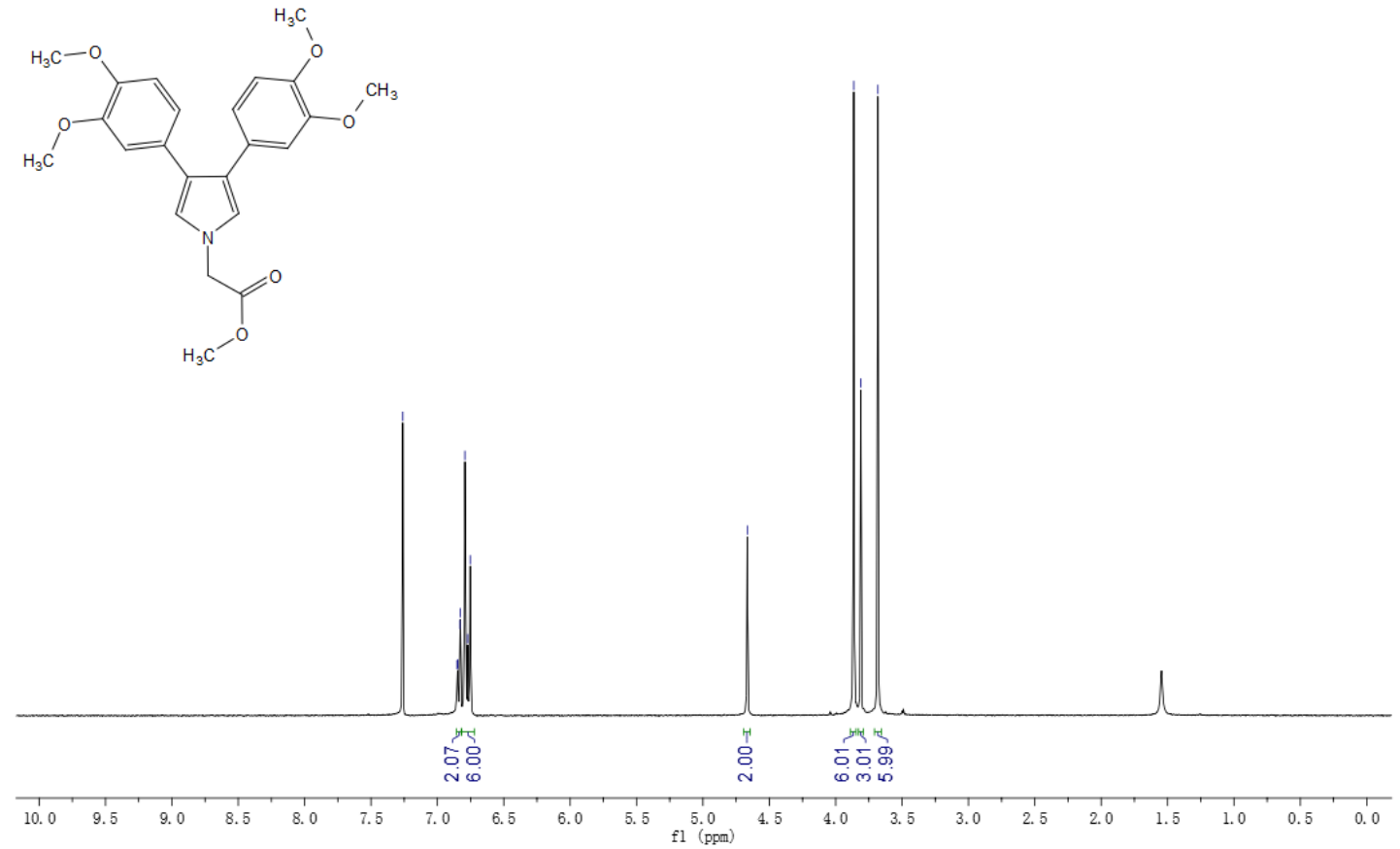

${ }^{13} \mathrm{C}$ NMR: 1

Solvent: $\mathrm{CDCl}_{3}$

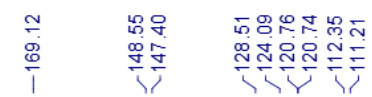

舟

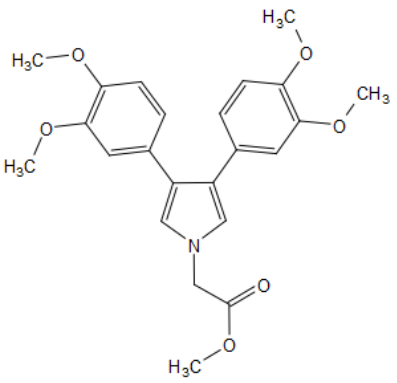

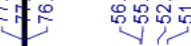

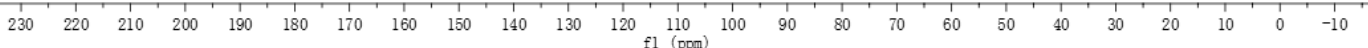




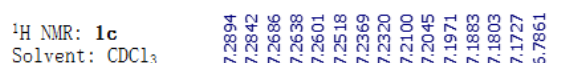

Solvent: $\mathrm{CDCl}_{3}$

$\underset{\substack{1 \\ 6}}{\substack{0 \\ 0}}$
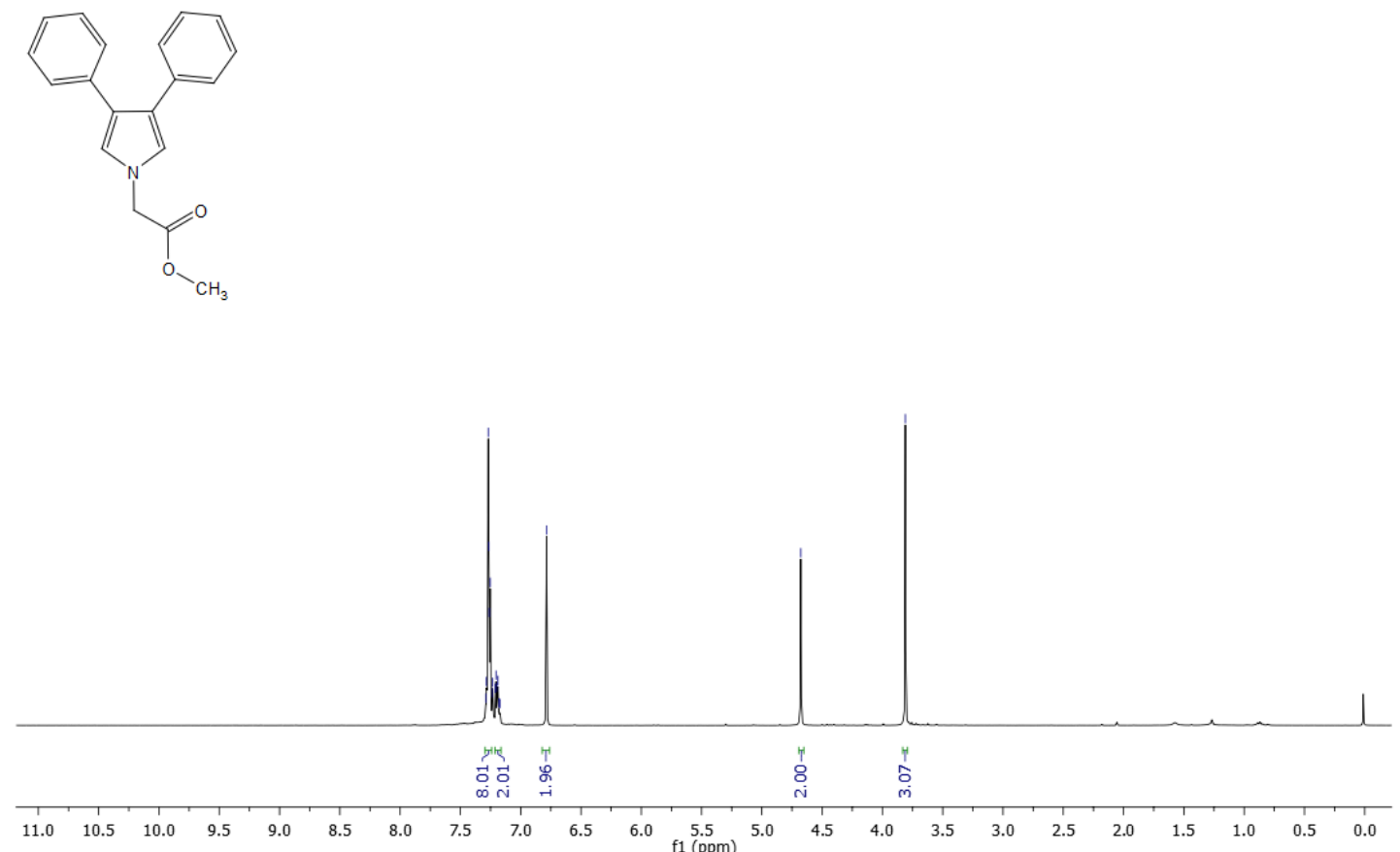

${ }^{13} \mathrm{C}$ NMR: 1c

Solvent: $\mathrm{CDCl}_{3}$

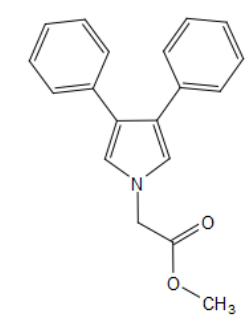

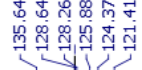

娒 解

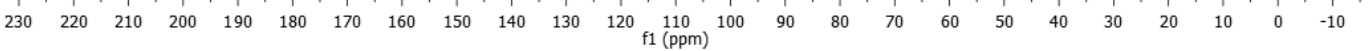


${ }^{1} \mathrm{H}$ NMR: 1d
Solvent: $C D C{ }^{3}$

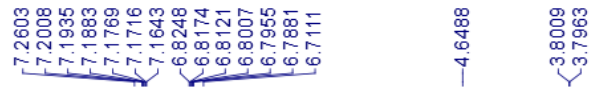

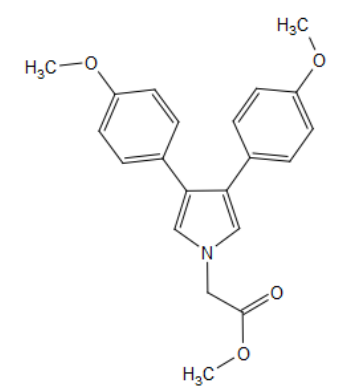

年

总

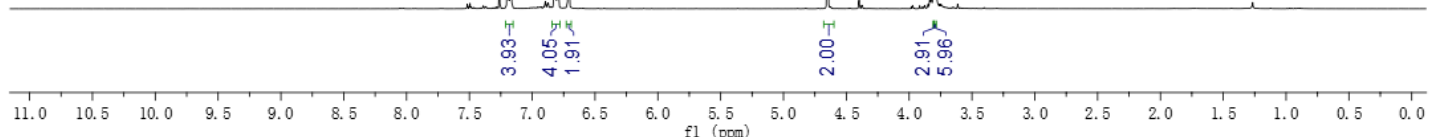

${ }^{13} \mathrm{C}$ NIR: $1 \mathrm{~d}$
Solvent: $\mathrm{CDCl}_{3}$

$\underset{\frac{1}{1}}{\frac{1}{0}} \frac{5}{5}$

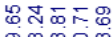

్ㅠㄲ뀸ำ

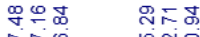

议尔

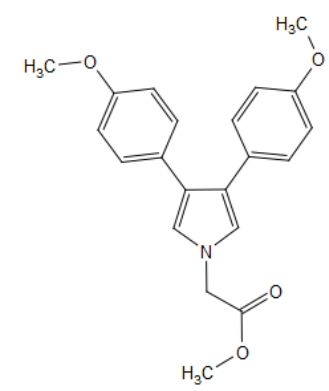

$\begin{array}{llllllllllllllllllllllllll}230 & 220 & 210 & 200 & 190 & 180 & 170 & 160 & 150 & 140 & 130 & 120 & 110 & 100 & 90 & 80 & 70 & 60 & 50 & 40 & 30 & 20 & 10 & 0 & -10\end{array}$ 


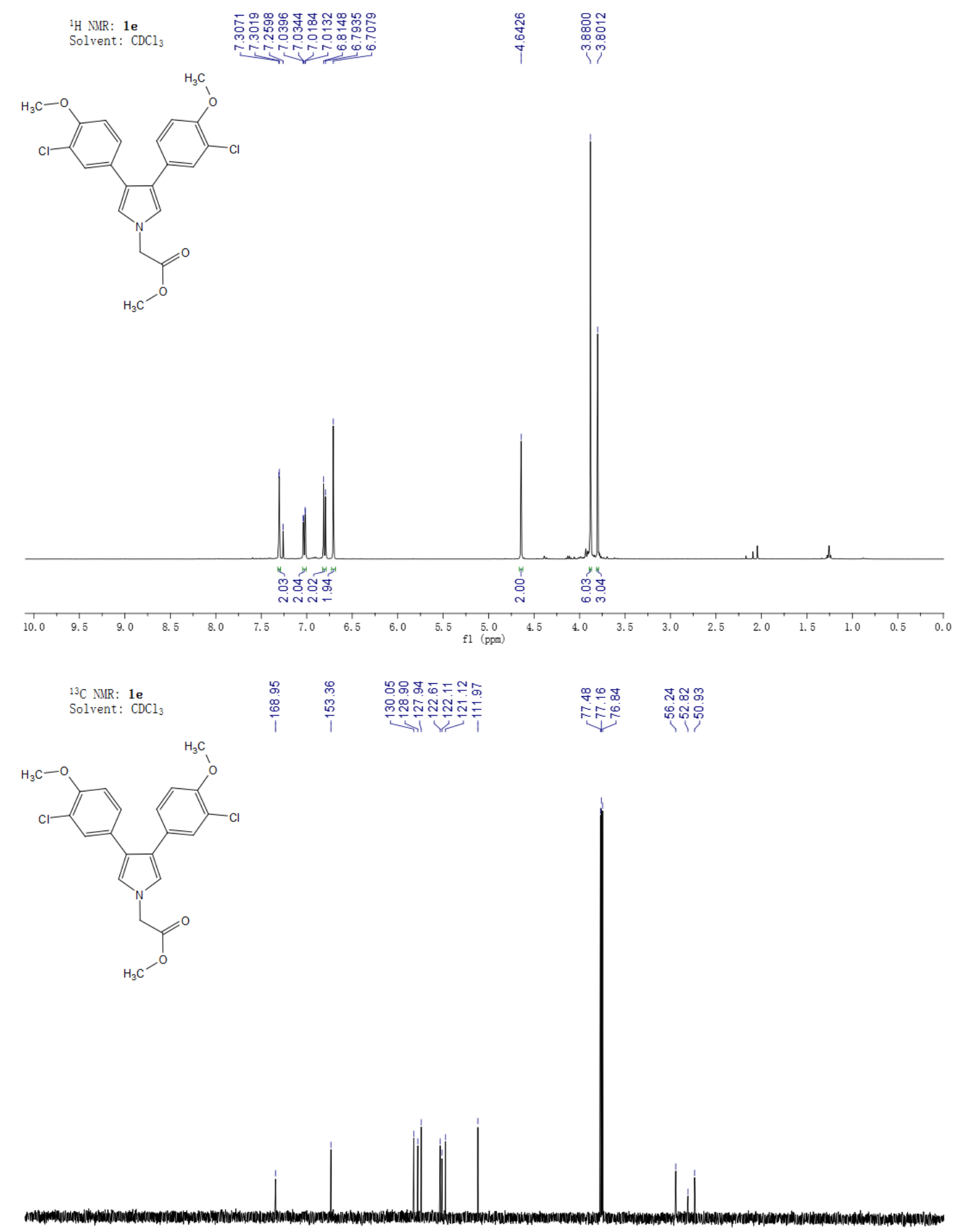

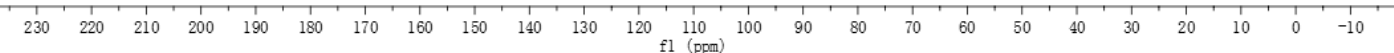




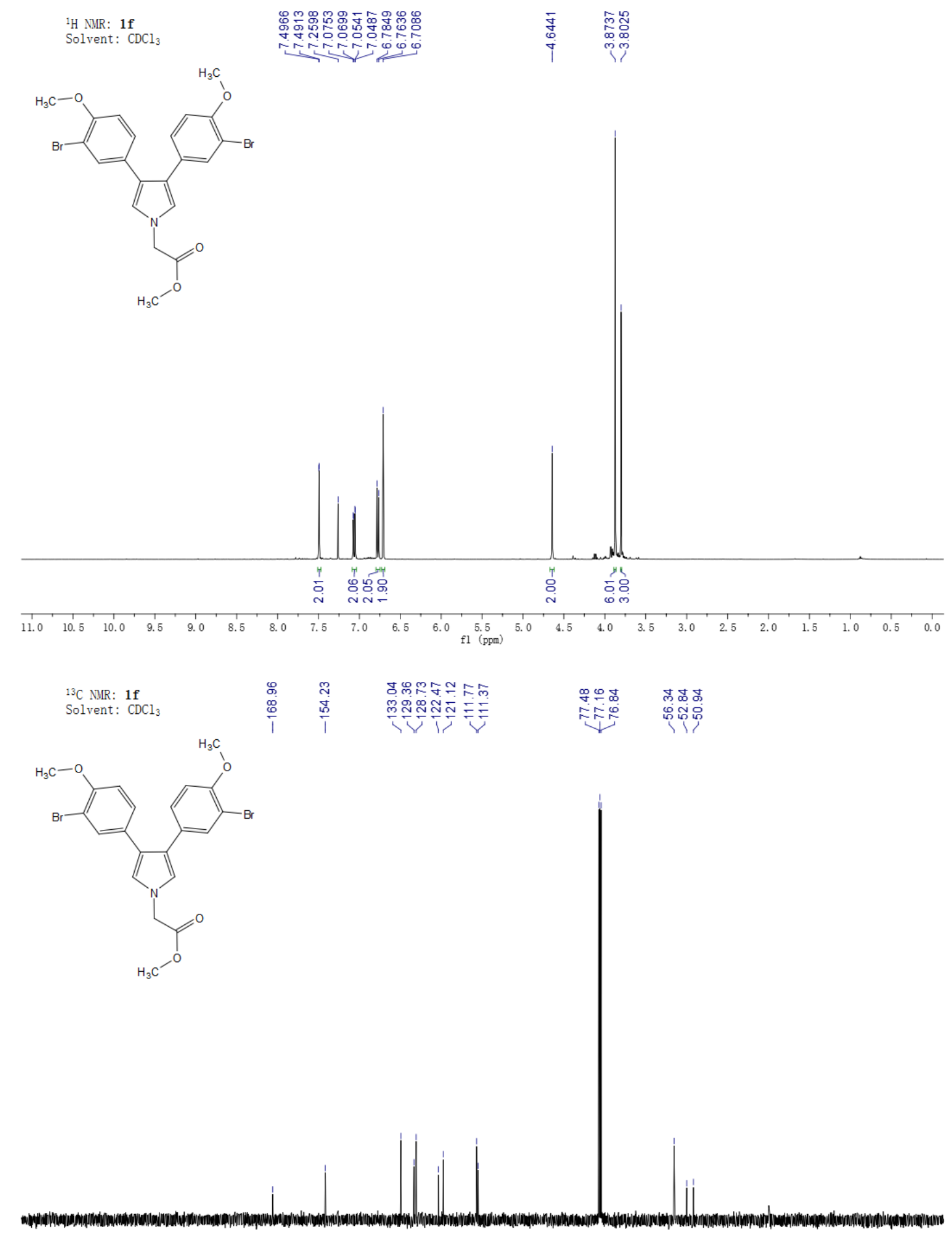

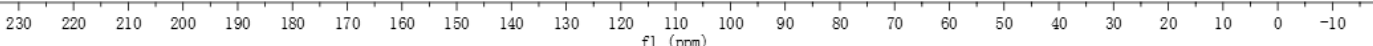




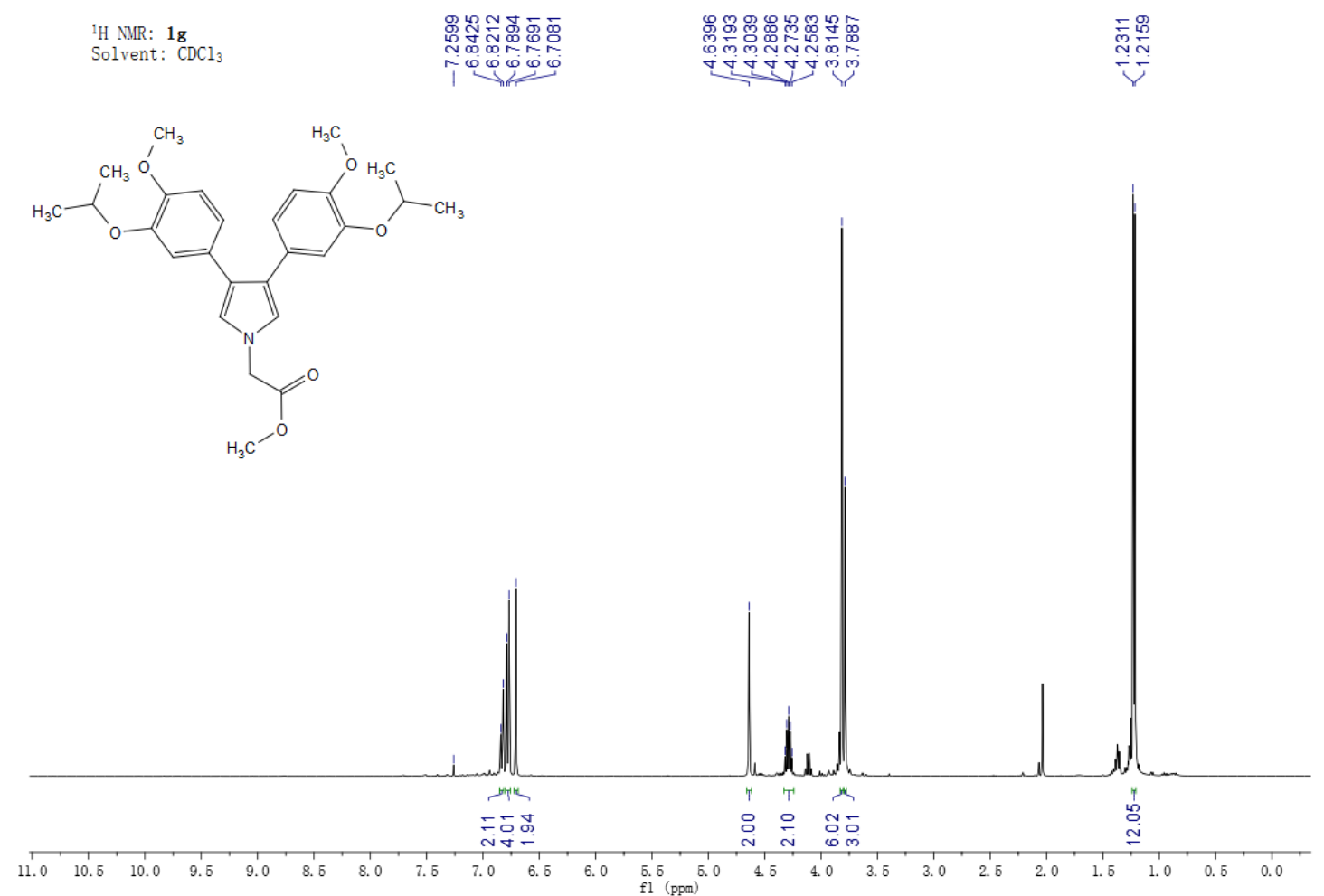

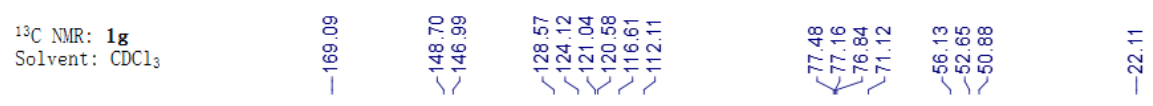

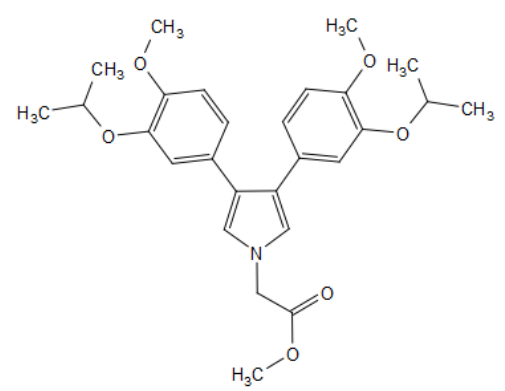

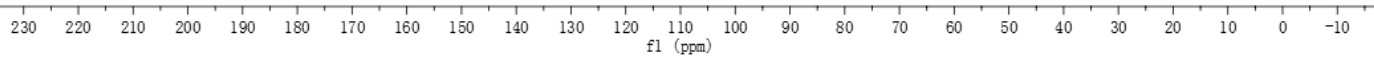



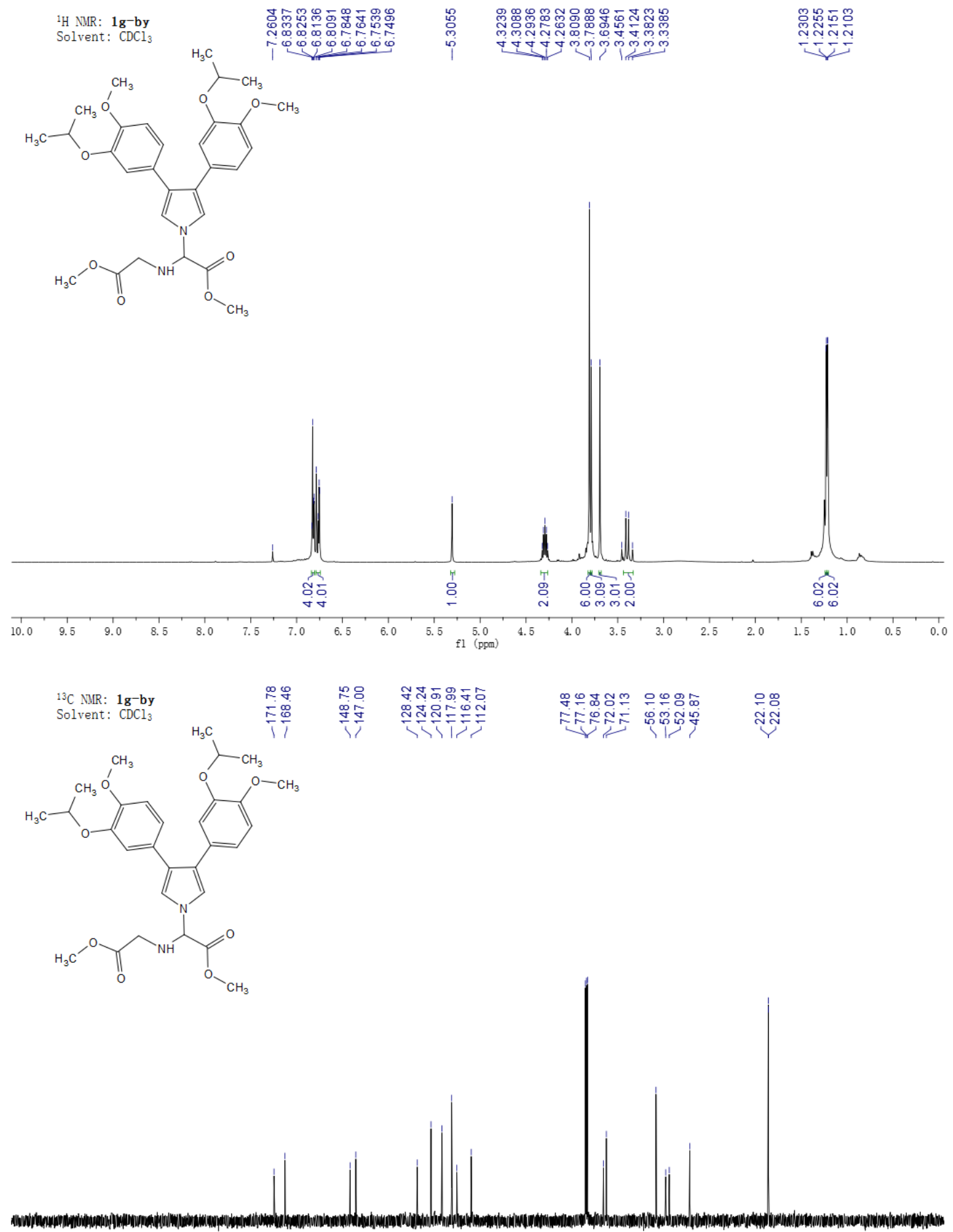

\begin{tabular}{rllllllllllllllllllllllllllllllll}
\hline 250 & 240 & 230 & 220 & 210 & 200 & 190 & 180 & 170 & 160 & 150 & 140 & 130 & 120 & 110 & 100 & 90 & 80 & 70 & 60 & 50 & 40 & 30 & 20 & 10 & 0 & -10 & -20 & -30
\end{tabular} 
${ }^{1} \mathrm{H}$ NMR: 2b

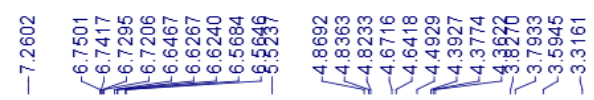

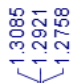
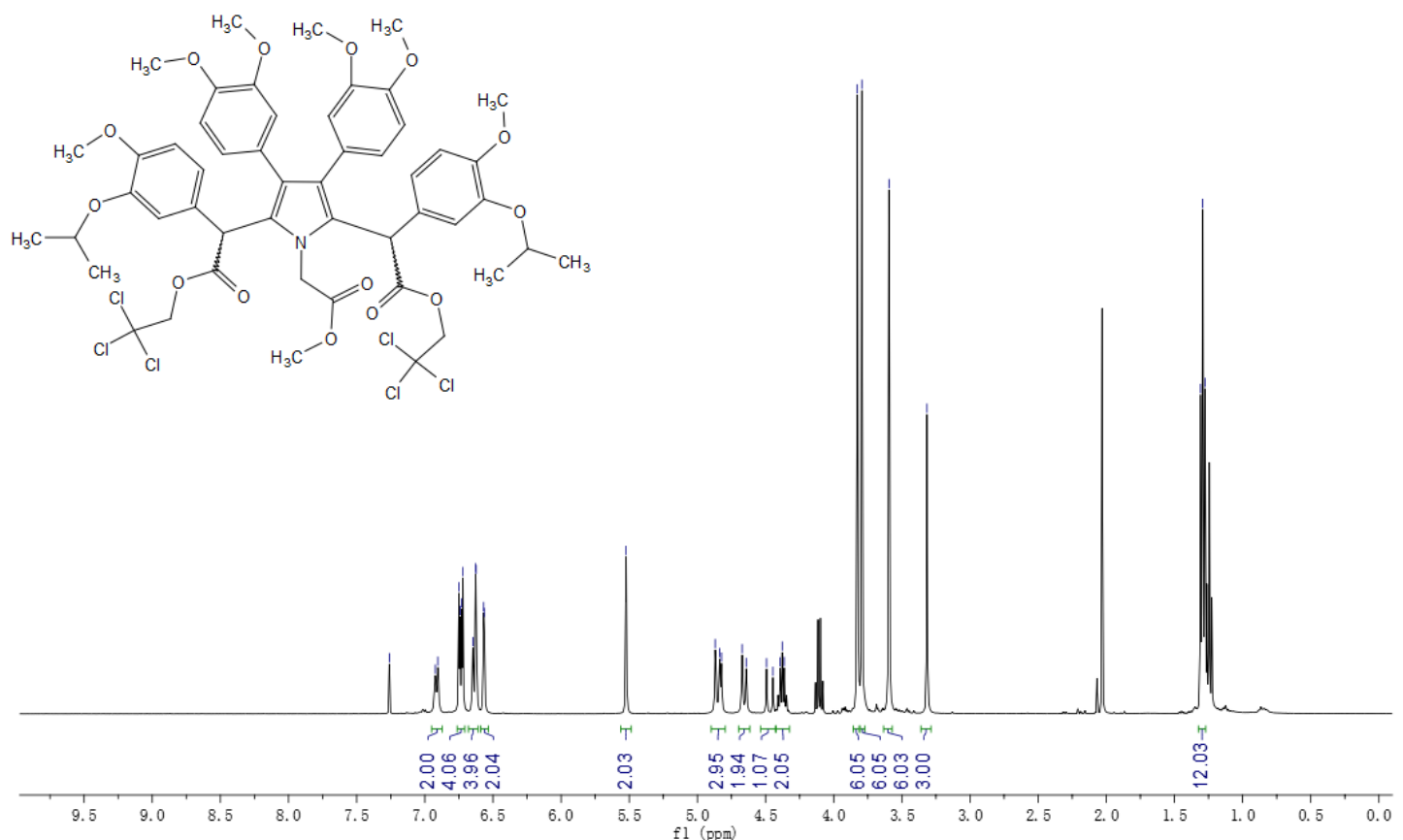

${ }^{13} \mathrm{C}$ NMR: $2 \mathrm{~b}$

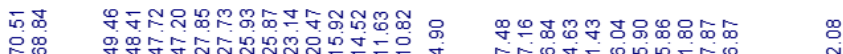

家

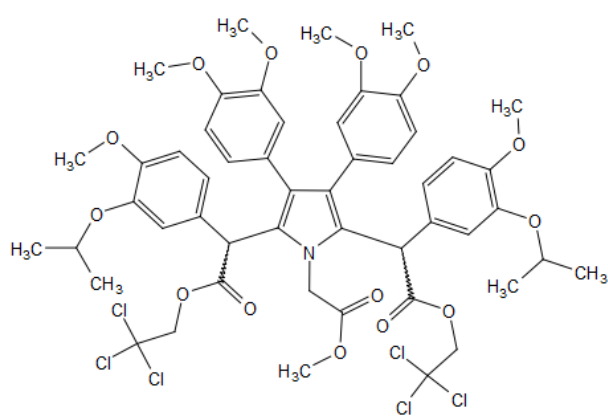

Solvent: $\mathrm{CDCl}_{3}$ 


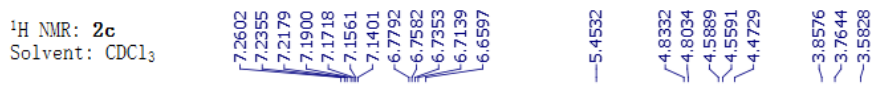<smiles>COC(=O)Cn1c(C(C(=O)OCC(Cl)(Cl)Cl)c2ccc(OC)c(OC)c2)c(-c2ccccc2)c(-c2ccccc2)c1C(C(=O)OCC(Cl)(Cl)Cl)c1ccc(OC)c(OC)c1</smiles>

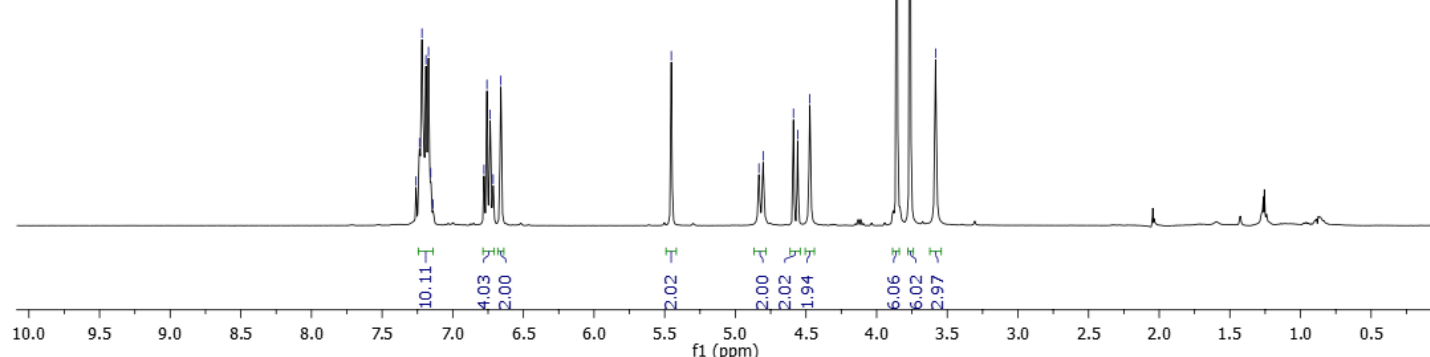

${ }^{13} \mathrm{C}$ NMR: $2 \mathrm{c}$

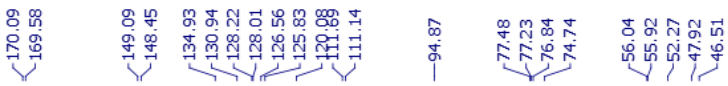
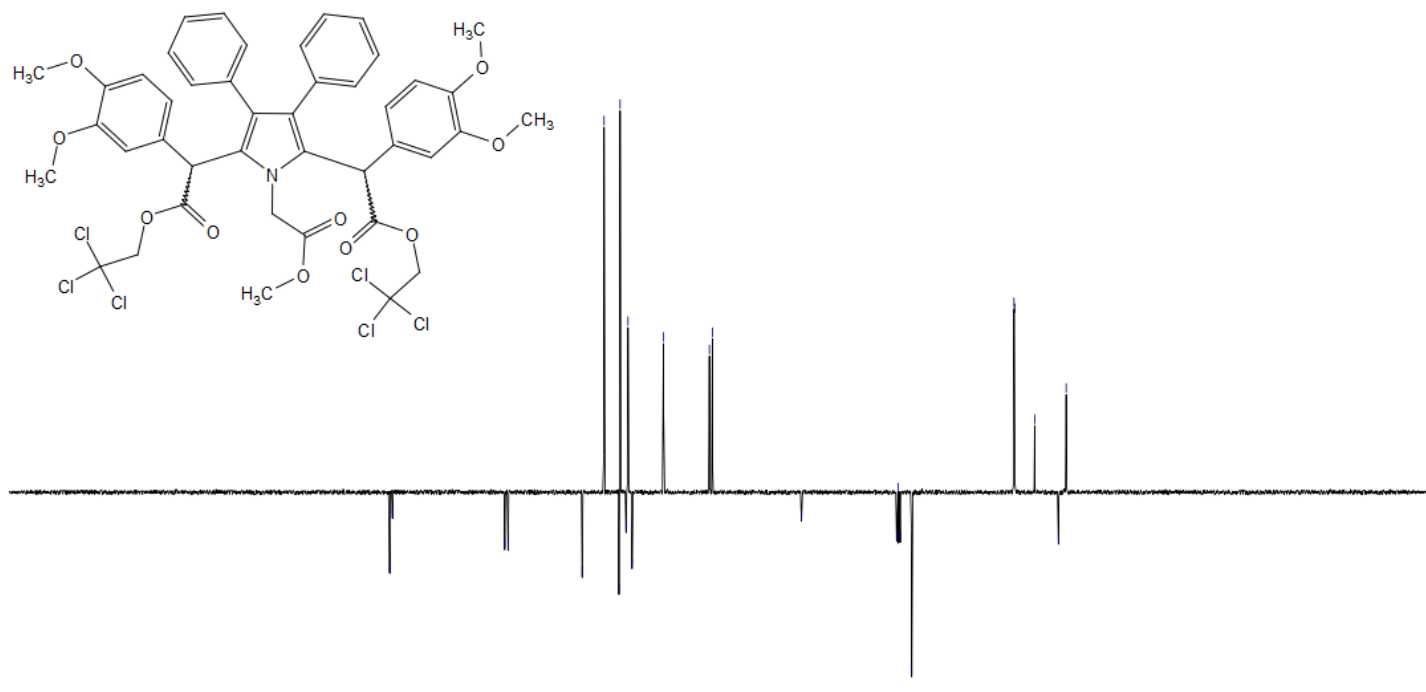

$\begin{array}{lllllllllllllllllllllllll}230 & 220 & 210 & 200 & 190 & 180 & 170 & 160 & 150 & 140 & 130 & 120 & \begin{array}{c}110 \\ \mathrm{fpm})\end{array} & 100 & 90 & 80 & 70 & 60 & 50 & 40 & 30 & 20 & 10 & 0 & -10\end{array}$ 


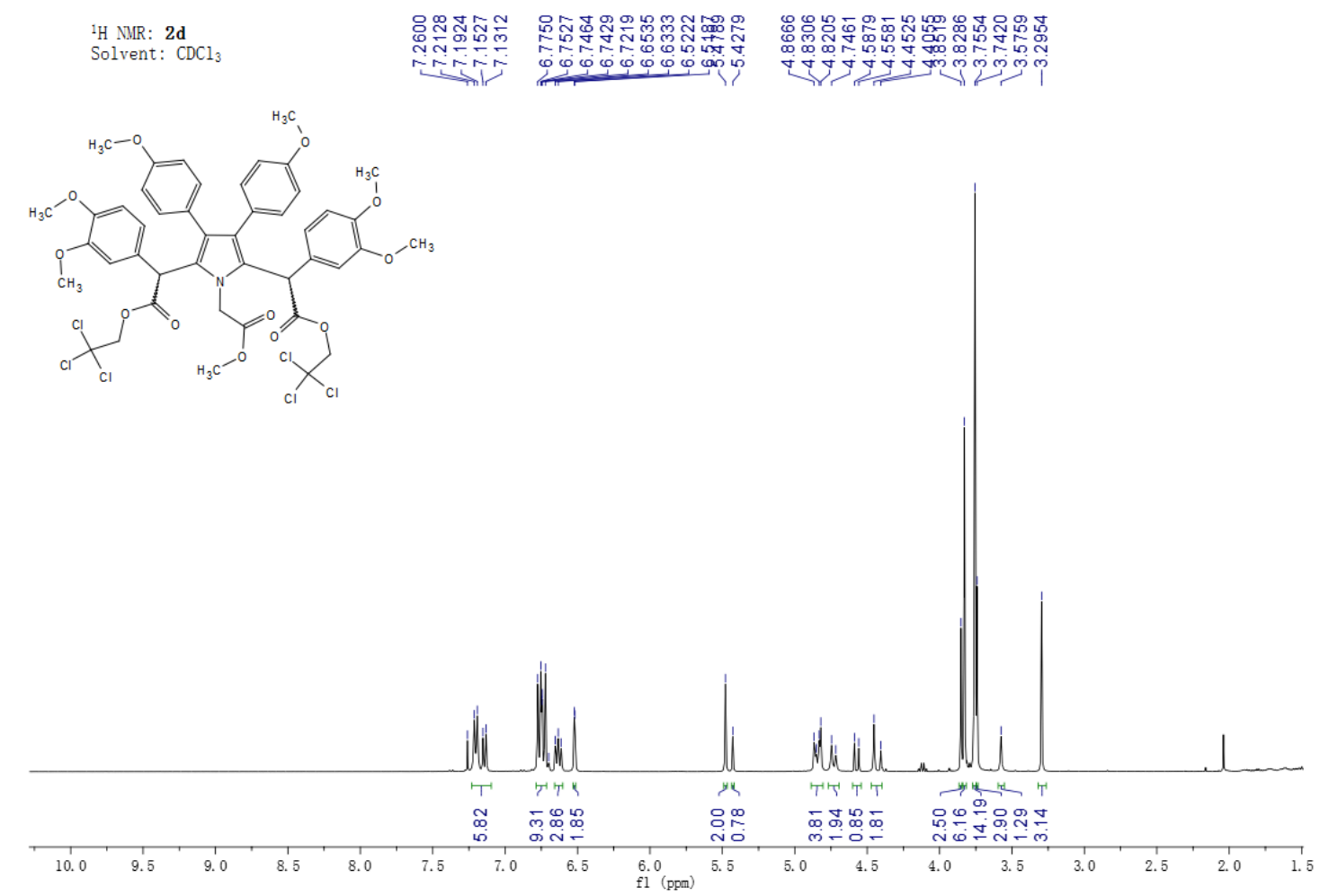

${ }^{13}$ C NMR: 2d Solvent: $\mathrm{CDCl}_{3}$<smiles>COC(=O)Cn1c(C(C(=O)OCC(Cl)(Cl)Cl)c2ccc(OC)c(OC)c2)c(-c2ccc(OC)cc2)c(-c2ccc(OC)cc2)c1C(C(=O)OCC(Cl)(Cl)Cl)c1ccc(OC)c(OC)c1</smiles>

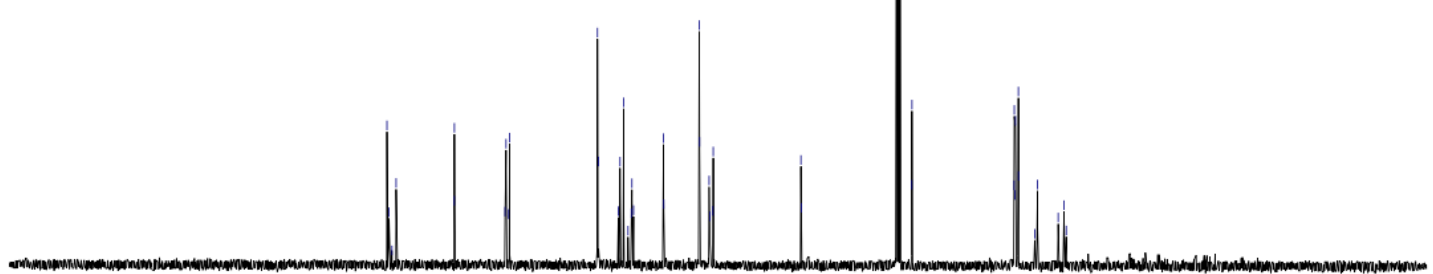

$\begin{array}{llllllllllllllllllllllllllllllllllllll}230 & 220 & 210 & 200 & 190 & 180 & 170 & 160 & 150 & 140 & 130 & 120 & 110 & 100 & 90 & 80 & 70 & 60 & 50 & 40 & 30 & 20 & 10 & 0 & -10\end{array}$ 


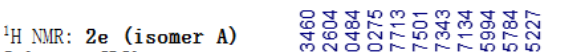

Solvent: $\mathrm{CDCl}$

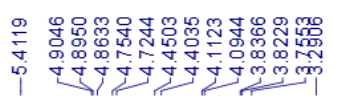

i<smiles>COC(=O)Cn1c(C(C(=O)OCC(Cl)(Cl)Cl)c2ccc(OC)c(Cl)c2)c(-c2ccc(OC)c(OC)c2)c(-c2ccc(OC)c(Cl)c2)c1C(C(=O)OCC(Cl)(Cl)Cl)c1ccc(OC)c(OC)c1</smiles>

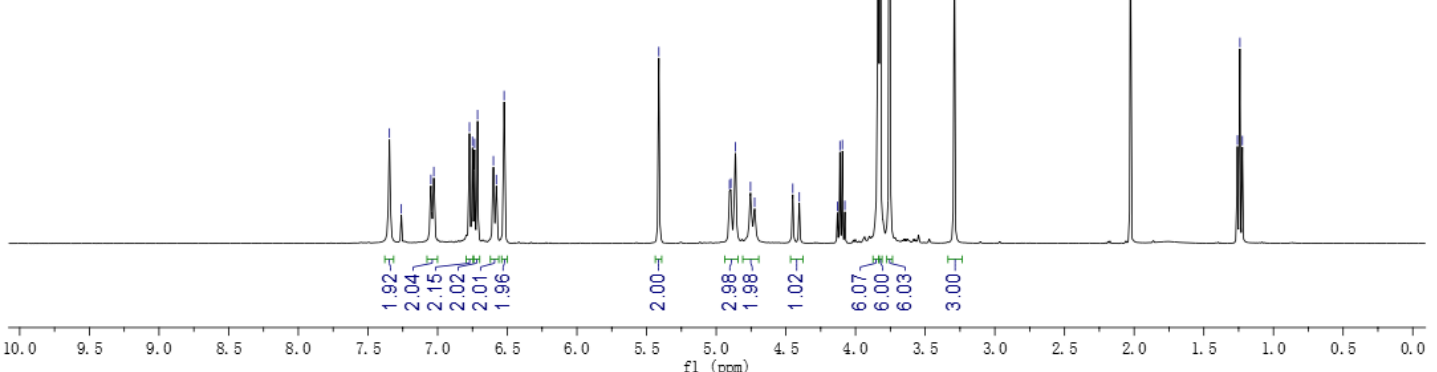

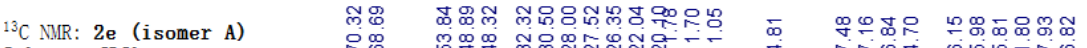

Solvent: $\mathrm{CDCl}_{3}$ (isomer A)<smiles>COC(=O)Cn1c(-c2ccc(OC)c(Cl)c2)c(-c2ccc(OC)c(Cl)c2)c(C(C(=O)OCC(Cl)(Cl)Cl)c2ccc(OC)c(OC)c2)c1C(C(=O)OCC(Cl)(Cl)Cl)c1ccc(OC)c(OC)c1</smiles>

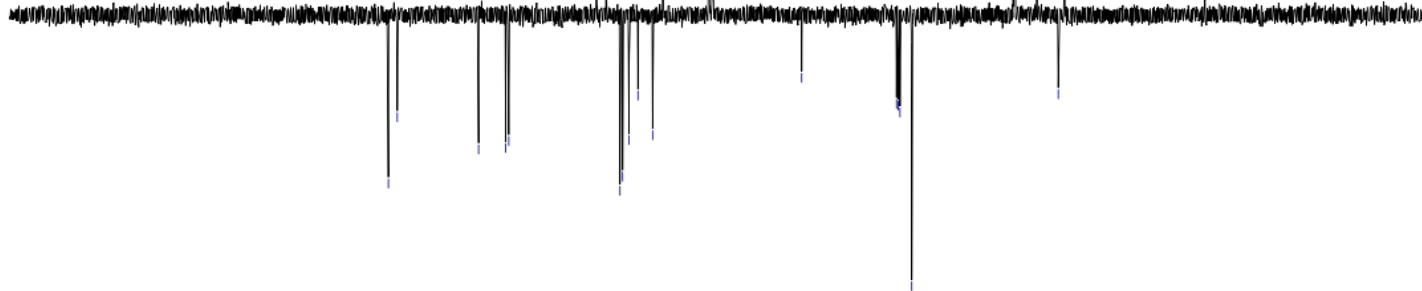

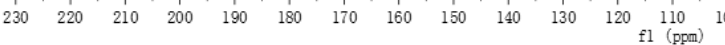




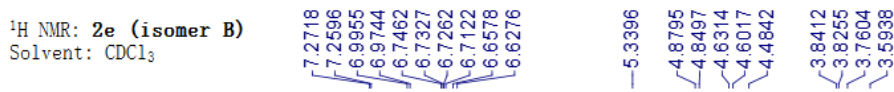<smiles>COC(=O)Cn1c(C(C(=O)OCC(Cl)(Cl)Cl)c2ccc(OC)c(Cl)c2)c(-c2ccc(OC)c(Cl)c2)c(-c2ccc(OC)c(OC)c2)c1C(C(=O)OCC(Cl)(Cl)Cl)c1ccc(OC)c(OC)c1</smiles>

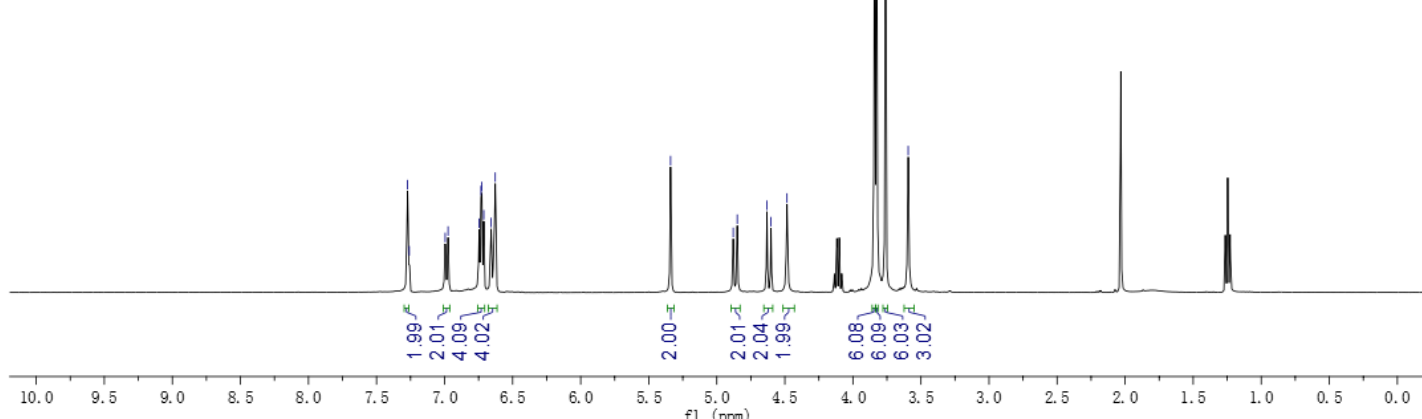

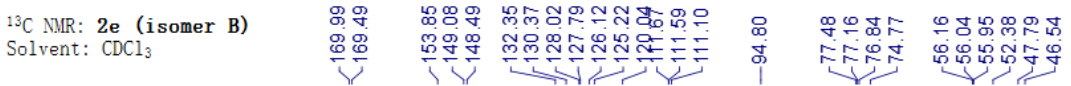<smiles>COC(=O)Cn1c(C(C(=O)OCC(Cl)(Cl)Cl)c2ccc(OC)c(Cl)c2)c(-c2ccc(OC)c(Cl)c2)c(-c2ccc(OC)c(OC)c2)c1C(C(=O)OCC(Cl)(Cl)Cl)c1ccc(OC)c(OC)c1</smiles>

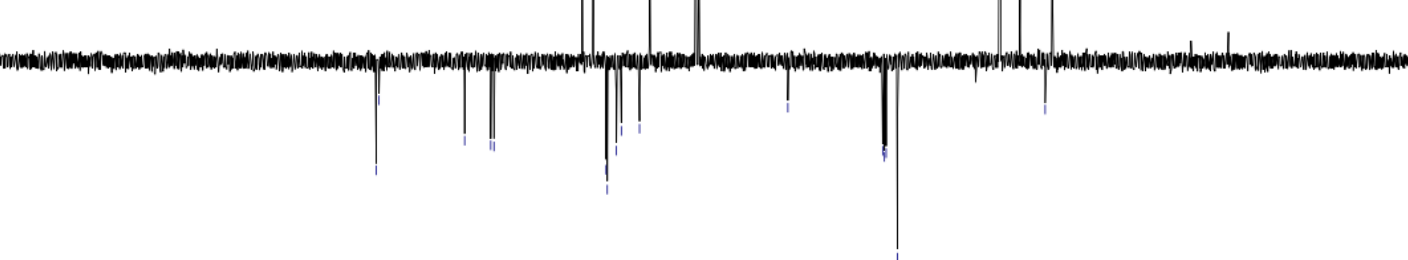

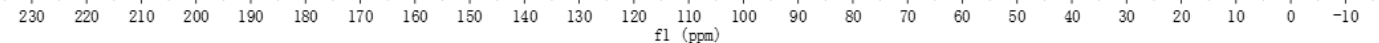


${ }^{1} \mathrm{H}$ NMR: $2 \mathrm{f}$
Solvent: $\mathrm{CDCl}$

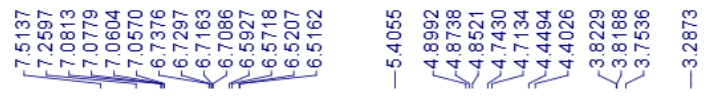<smiles>COC(=O)Cn1c(C(C(=O)OCC(Cl)(Cl)Cl)c2ccc(OC)c(OC)c2)c(-c2ccc(OC)c(Br)c2)c(-c2ccc(OC)c(Br)c2)c1C(C(=O)OCC(Cl)(Cl)Cl)c1ccc(OC)c(OC)c1</smiles>

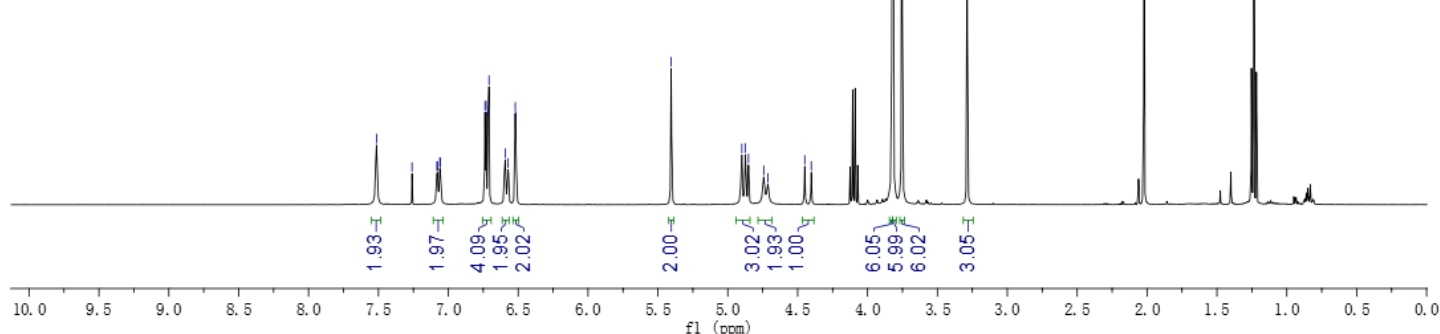

${ }^{13} \mathrm{C}$ NMR: $2 \mathrm{f}$
Solvent: $\mathrm{CDCl}$

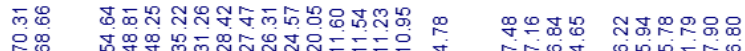

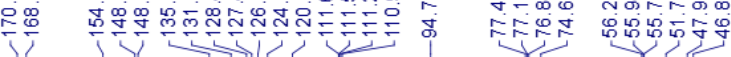<smiles>COC(=O)Cn1c(C(C(=O)OCC(Cl)(Cl)Cl)c2ccc(OC)c(Br)c2)c(-c2ccc(OC)c(Br)c2)c(-c2ccc(OC)c(OC)c2)c1C(C(=O)OCC(Cl)(Cl)Cl)c1ccc(OC)c(OC)c1</smiles>

$\begin{array}{llllllllllllll}230 & 220 & 210 & 200 & 190 & 180 & 170 & 160 & 150 & 140 & 130 & 120 & 110 & 100\end{array}$ 


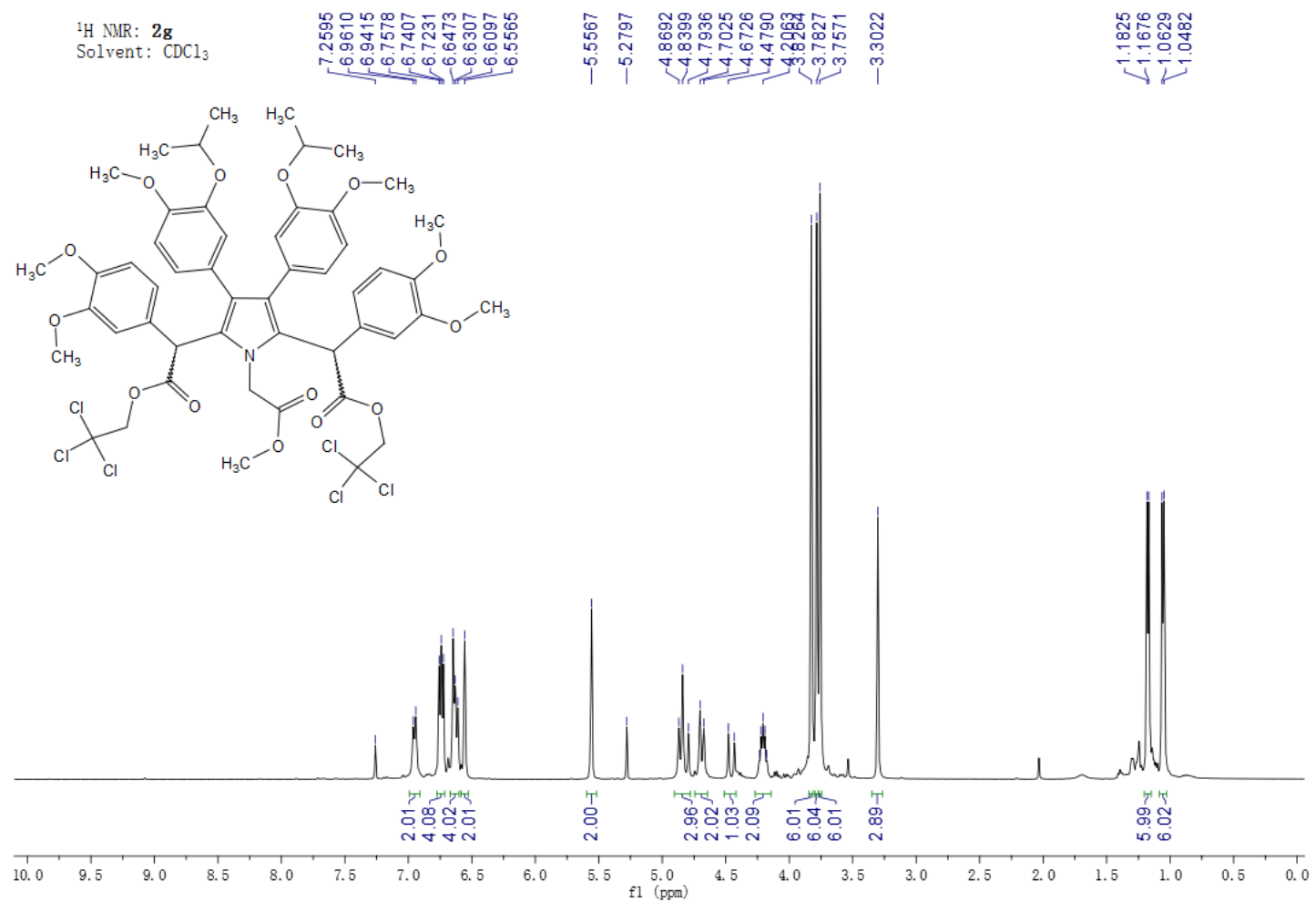

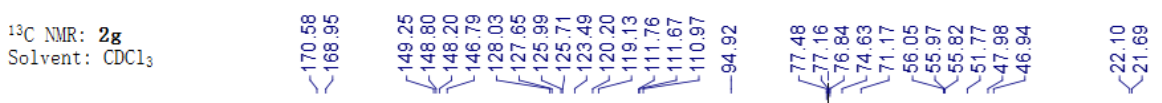
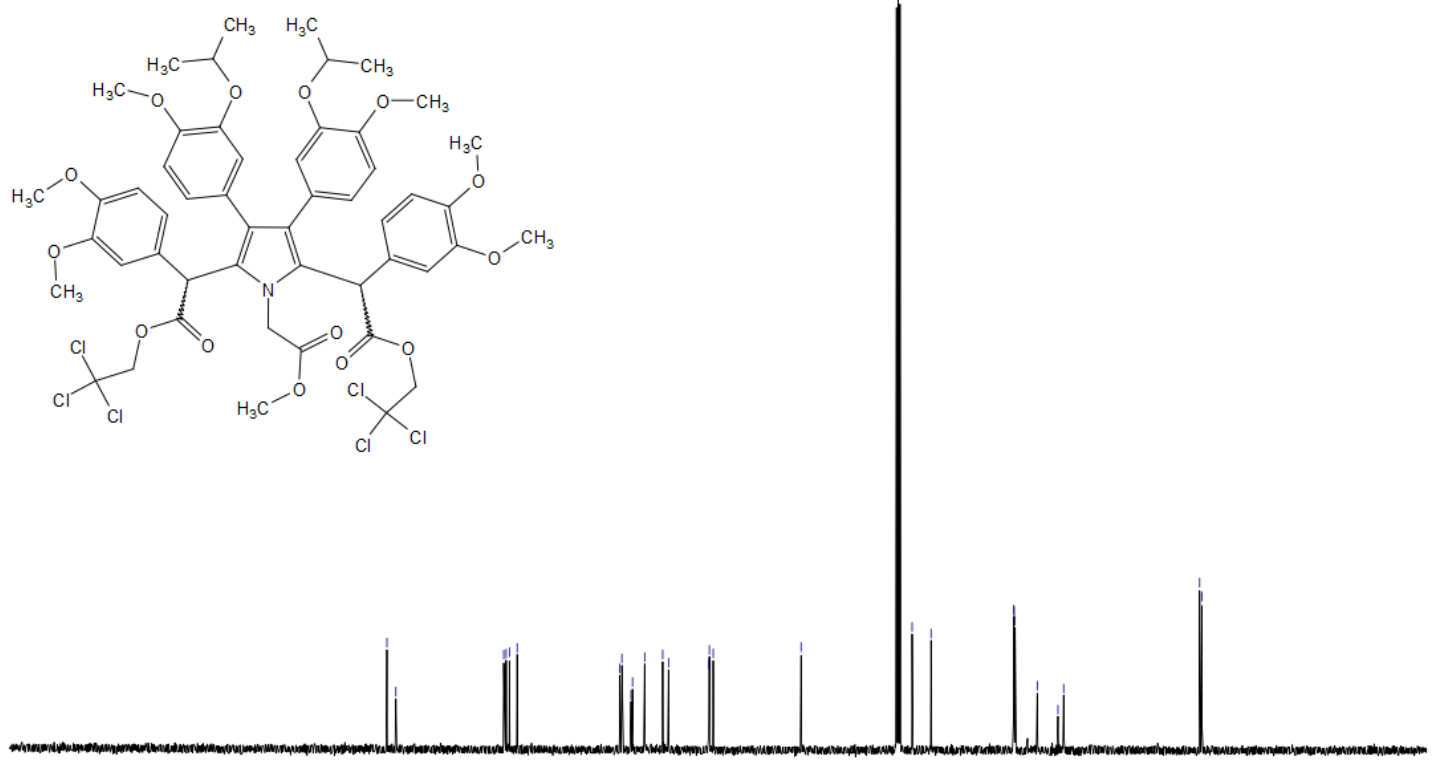

$\begin{array}{llllllllllllllllllllllllllllllllllll}230 & 220 & 210 & 200 & 190 & 180 & 170 & 160 & 150 & 140 & 130 & 120 & 110 & 100 & 90 & 80 & 70 & 60 & 50 & 40 & 30 & 20 & 10 & 0 & -10\end{array}$ 

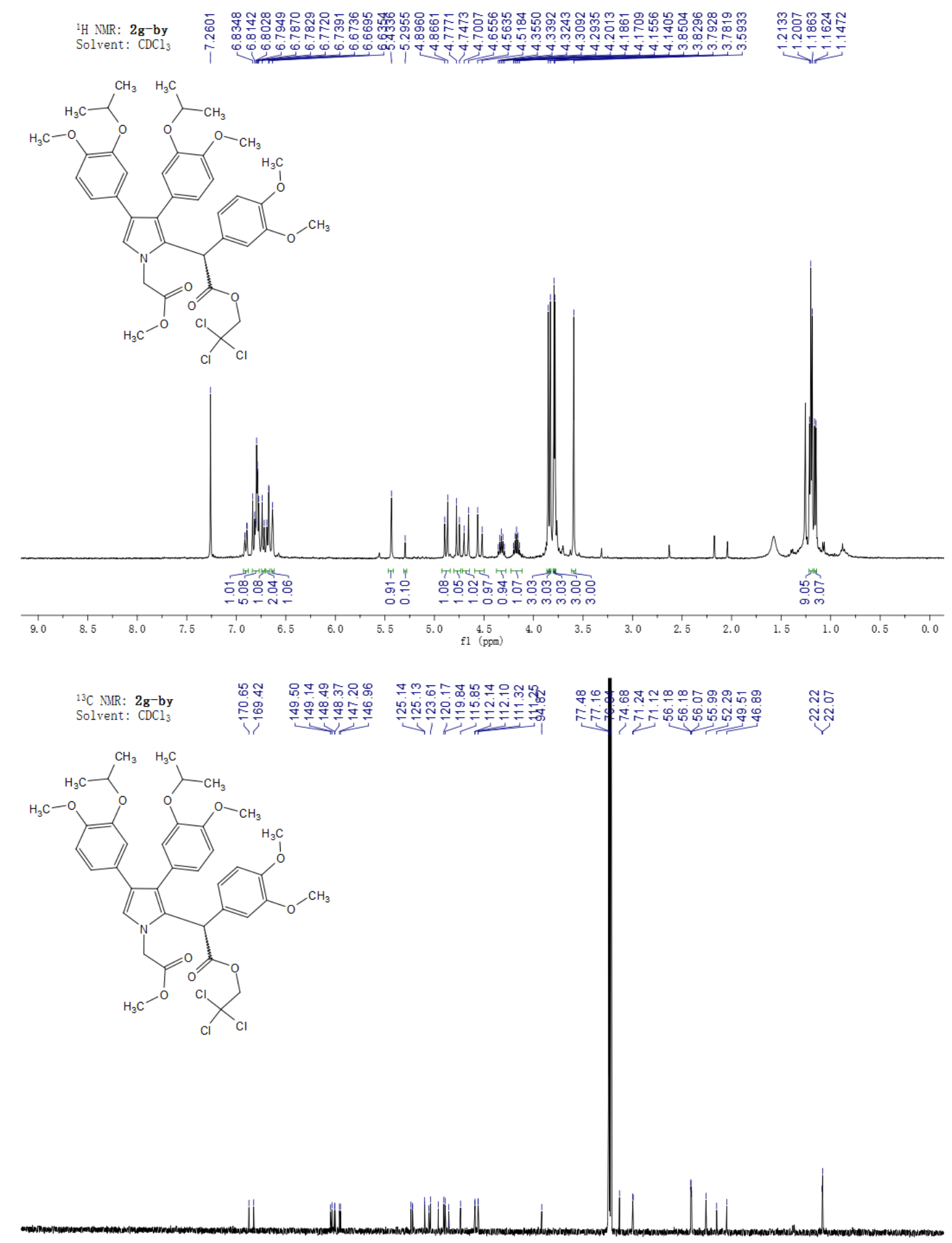

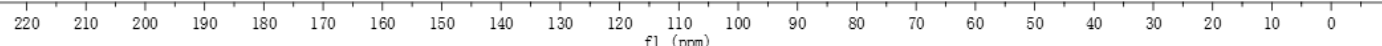



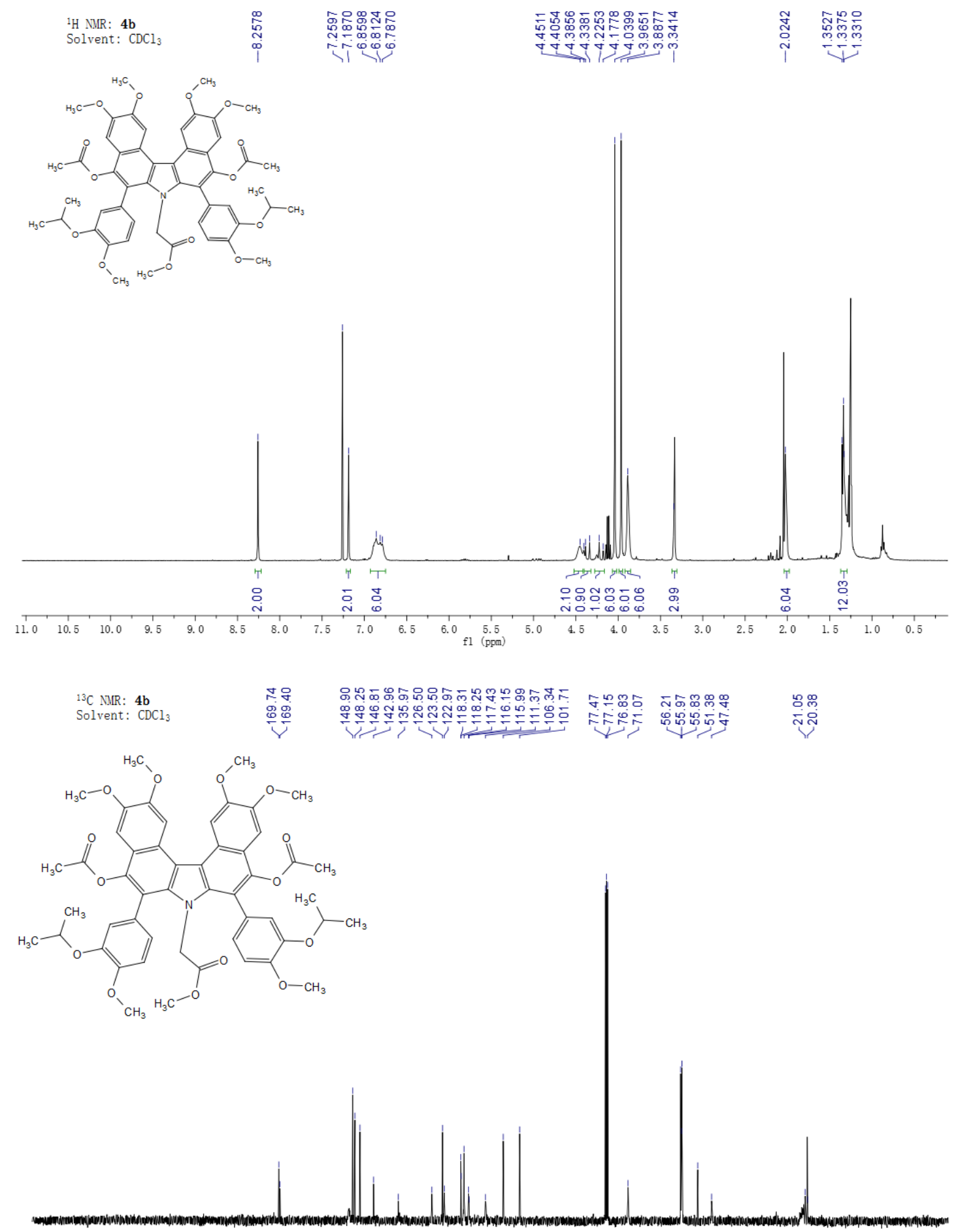

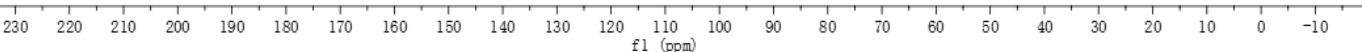




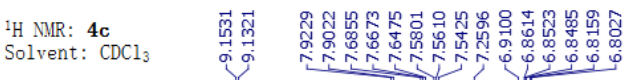

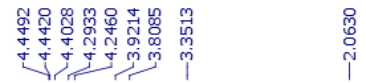
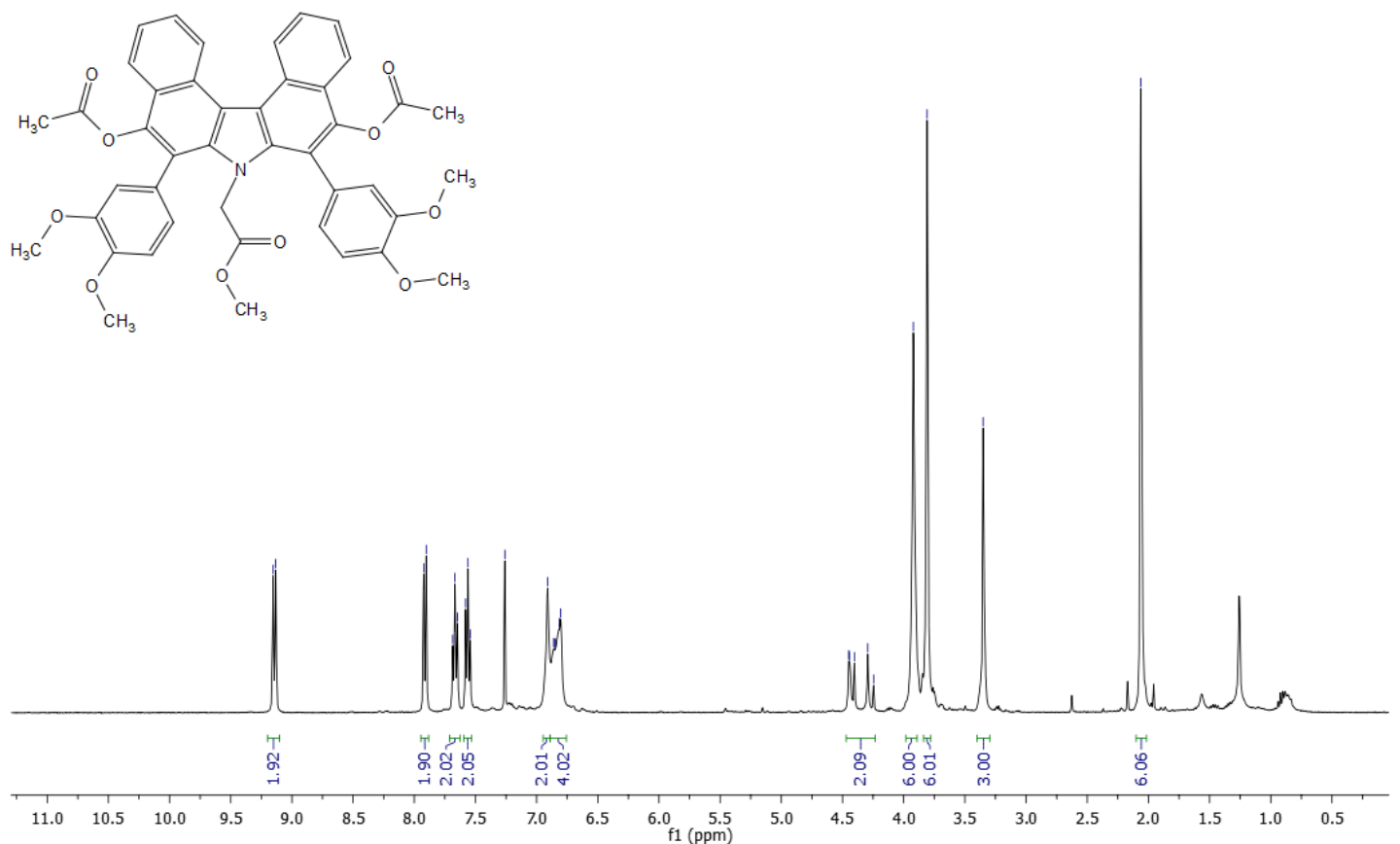

${ }^{13} \mathrm{C}$ NMR: $4 \mathbf{c}$
Solvent: $\mathrm{CDCl}_{3}$

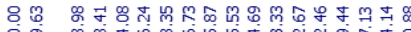

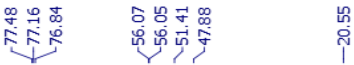

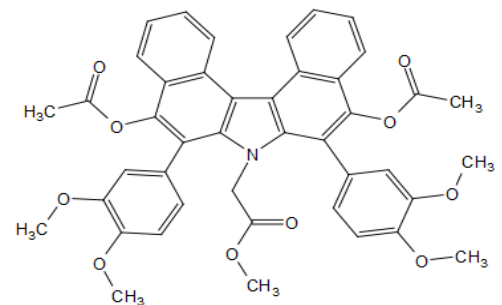

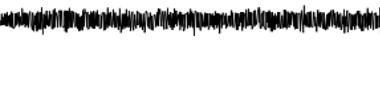

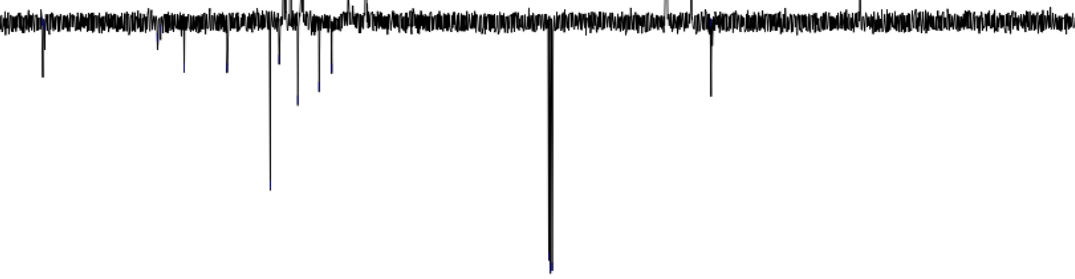

$\begin{array}{llllllllllll}230 & 220 & 210 & 200 & 190 & 180 & 170 & 160 & 150 & 140 & 130 & 120 \\ \mathrm{f} 1(\mathrm{ppm}) & 100\end{array}$ 
${ }^{1} \mathrm{H}$ NMR: 4d

Solvent: $\mathrm{CDCl}_{3}$
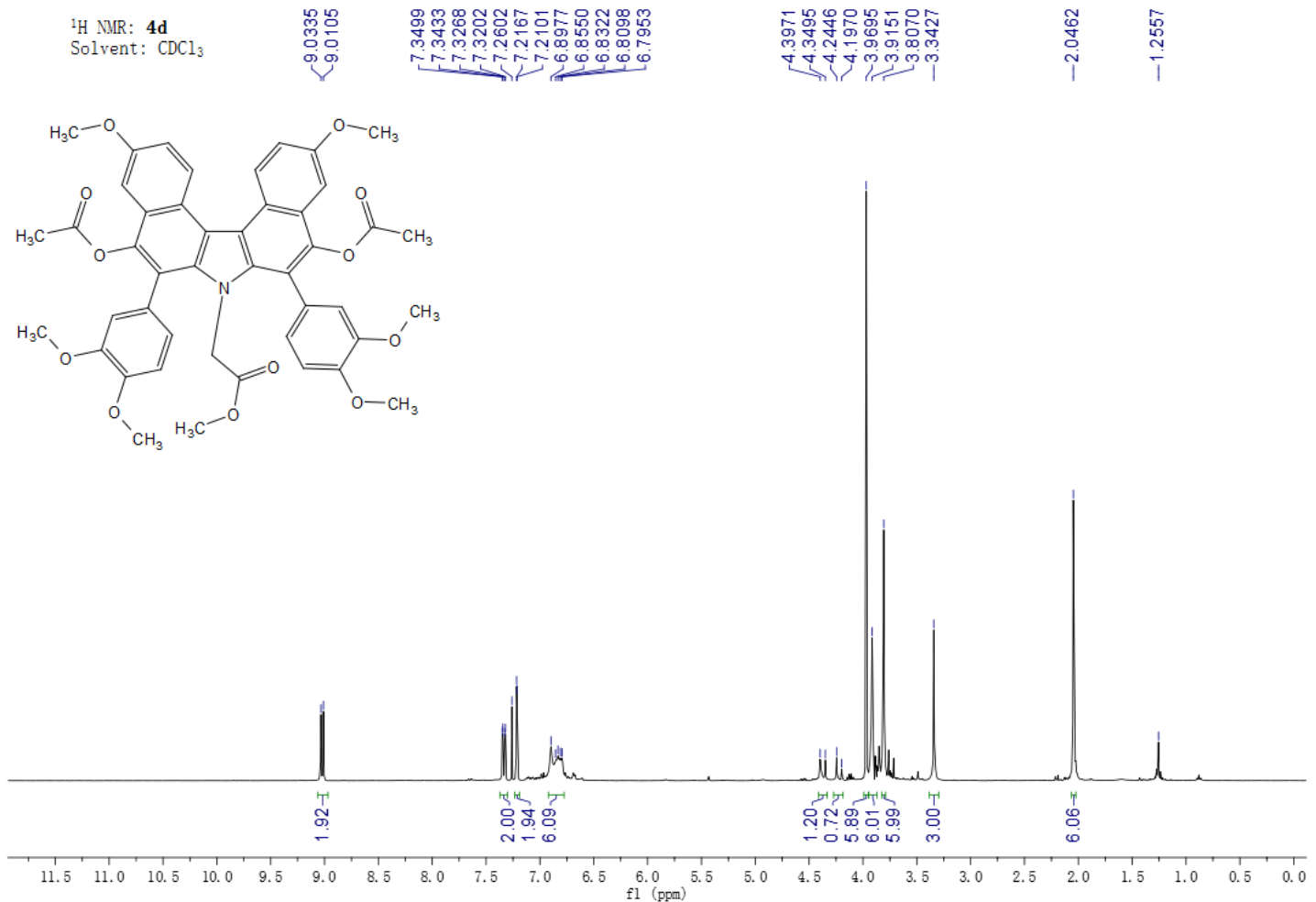

${ }^{13} \mathrm{C}$ NMR: 4d

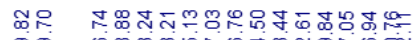

家

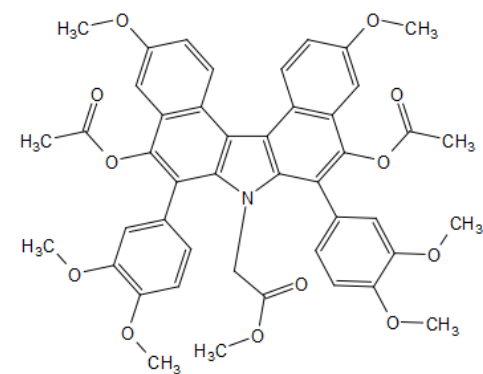

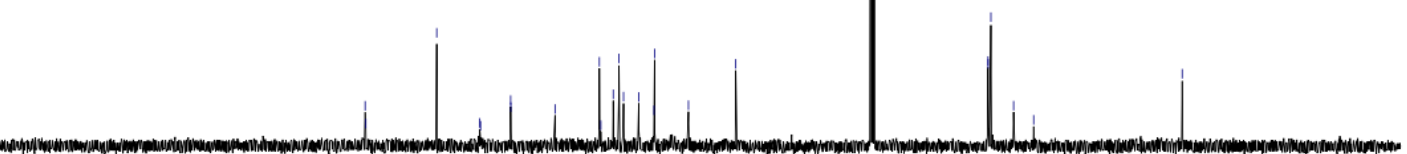

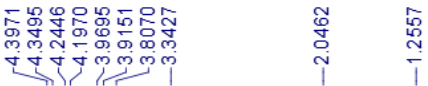




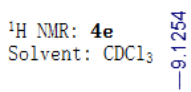

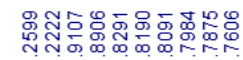

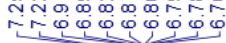

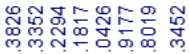

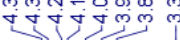

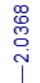
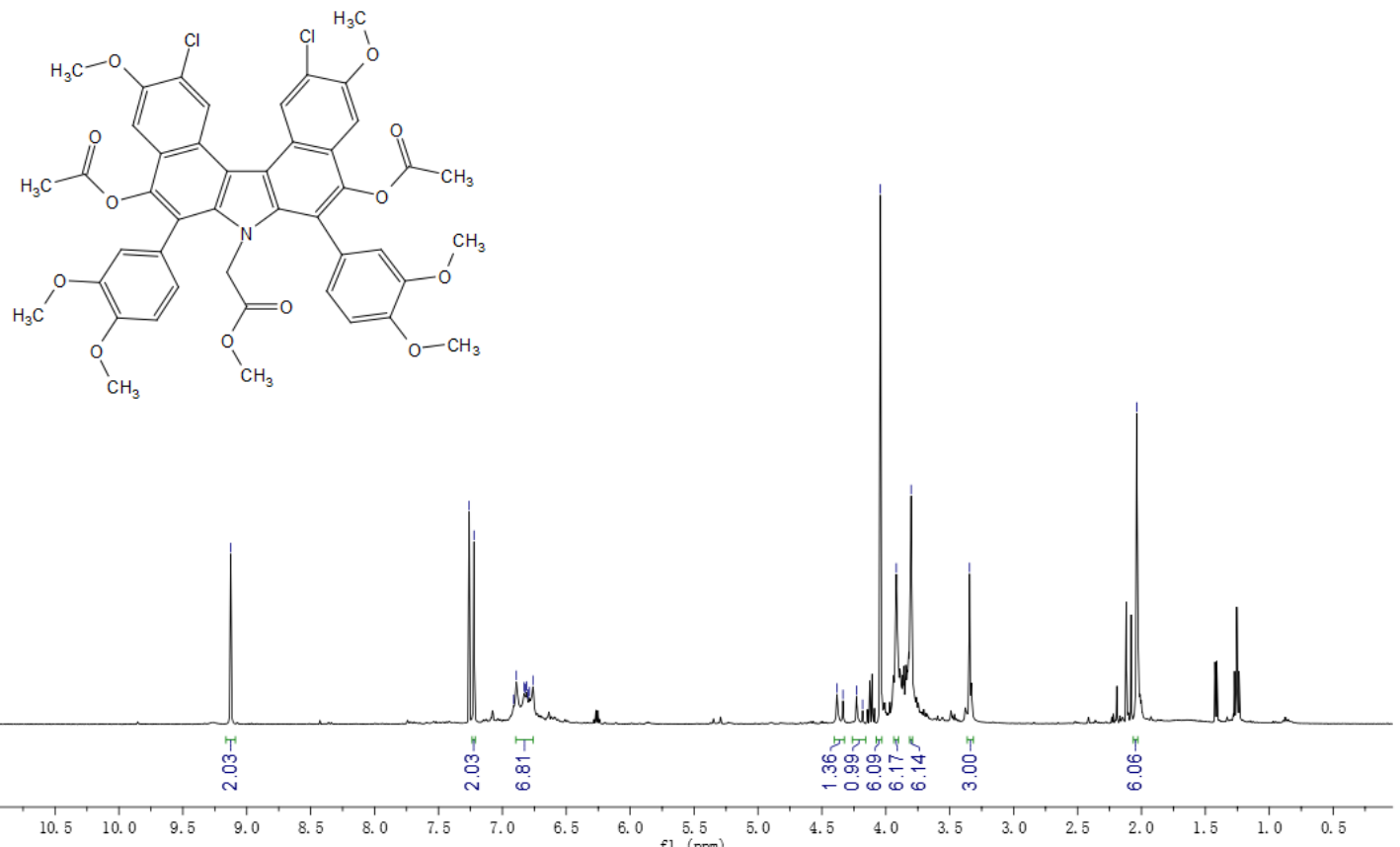

${ }^{13} \mathrm{C}$ NMR: $4 \mathrm{e}$
Solvent: $\mathrm{CDC}$

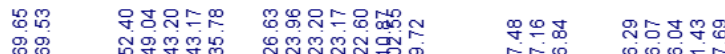

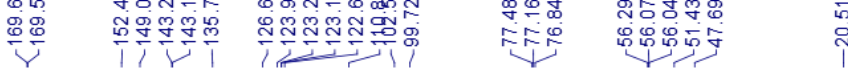
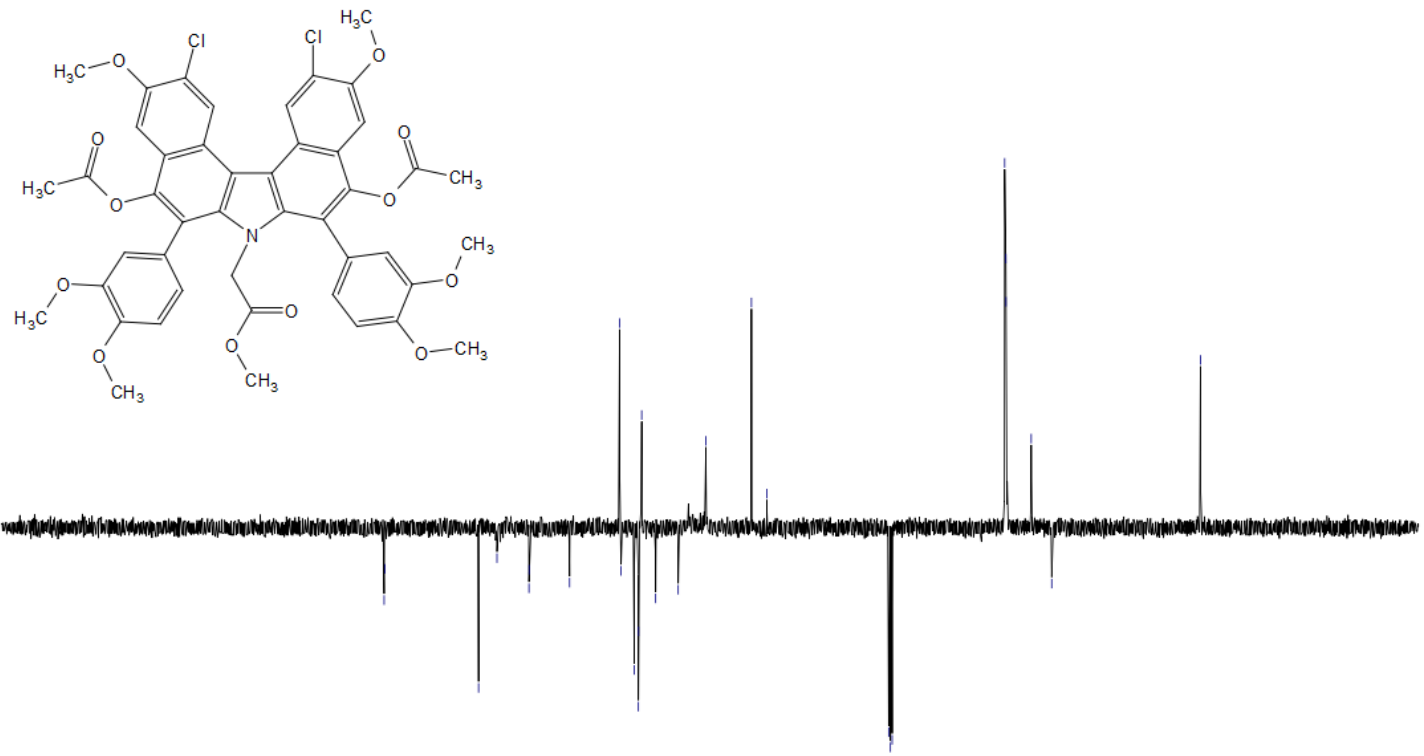

$230-220-210$

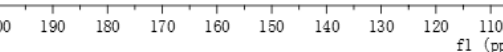

100 

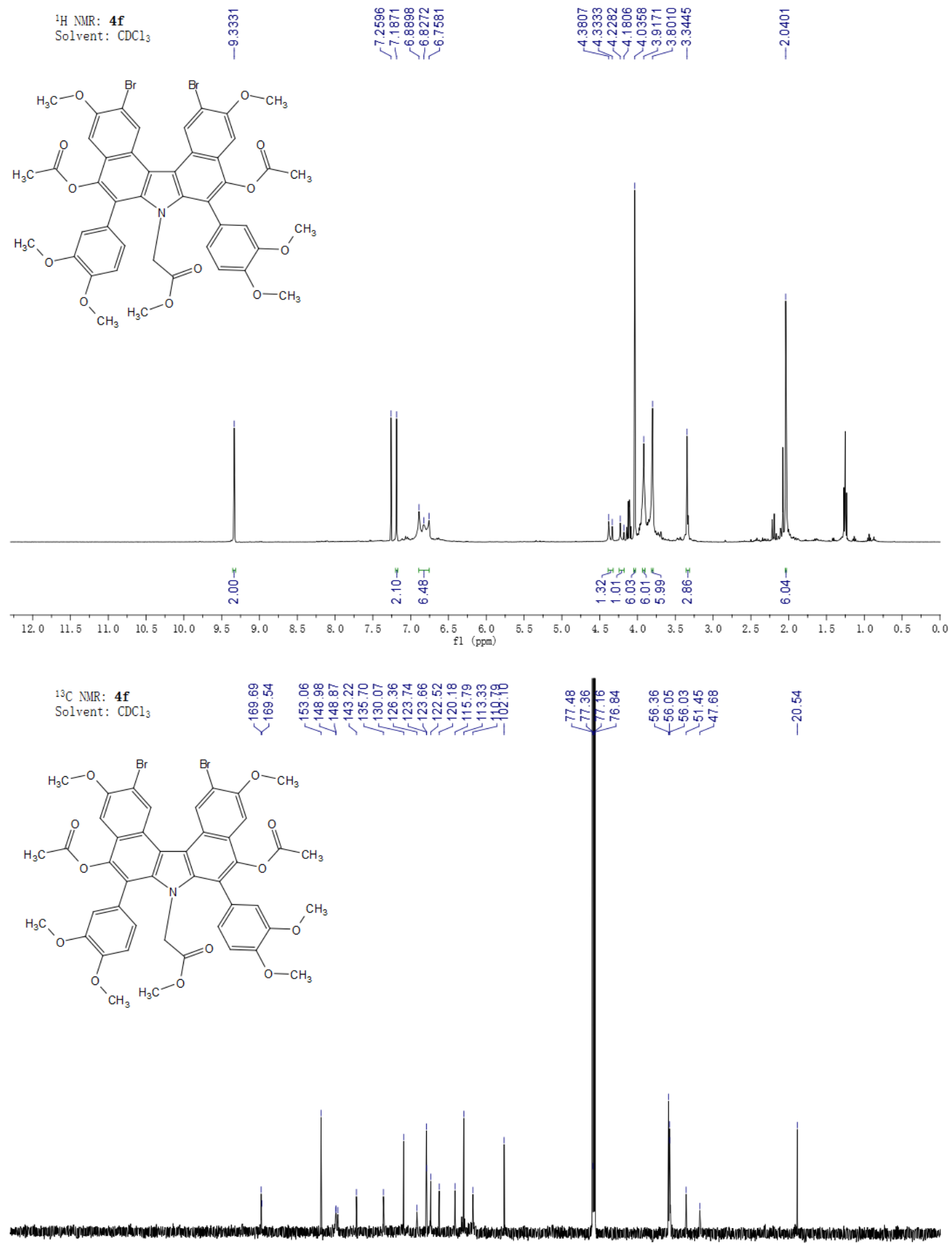

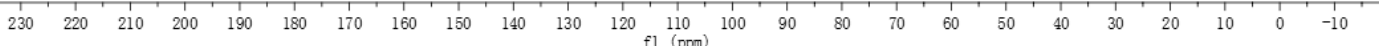


${ }^{1} \mathrm{H}$ NMR: $\mathbf{4 g}$
Solvent: $\mathrm{CDCl}_{3}$
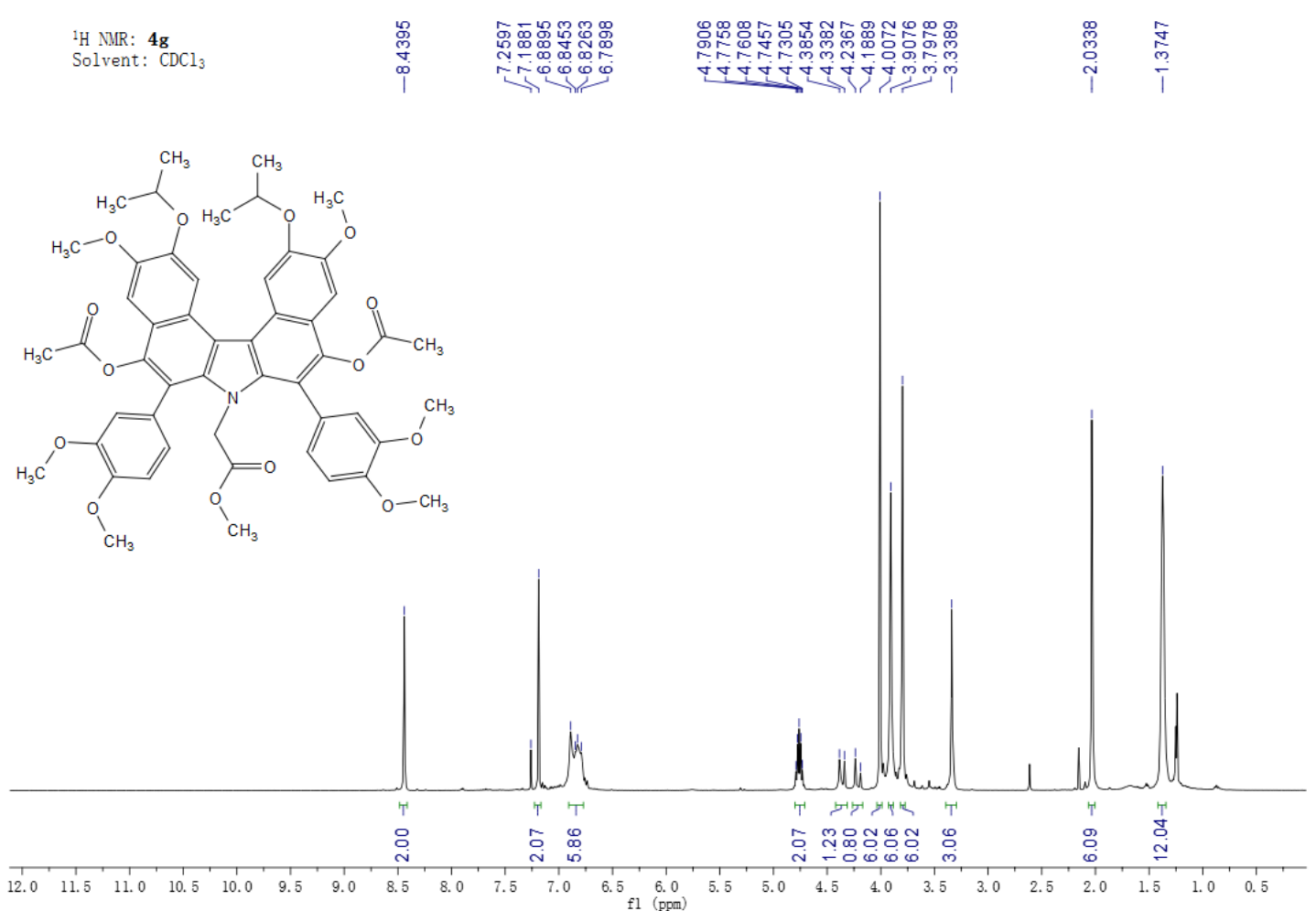

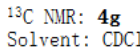

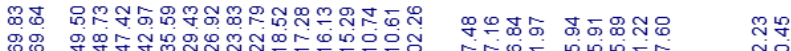

๓⿴囗十

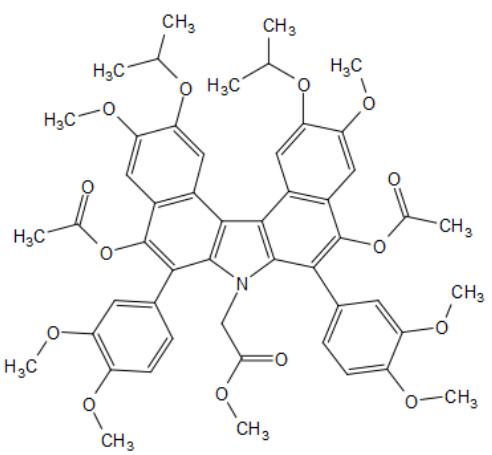

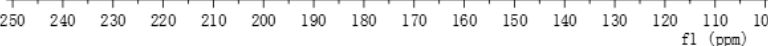


1H NMR: II-135

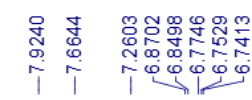

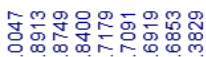

lvent: $\mathrm{CDCl}_{3}$

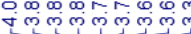
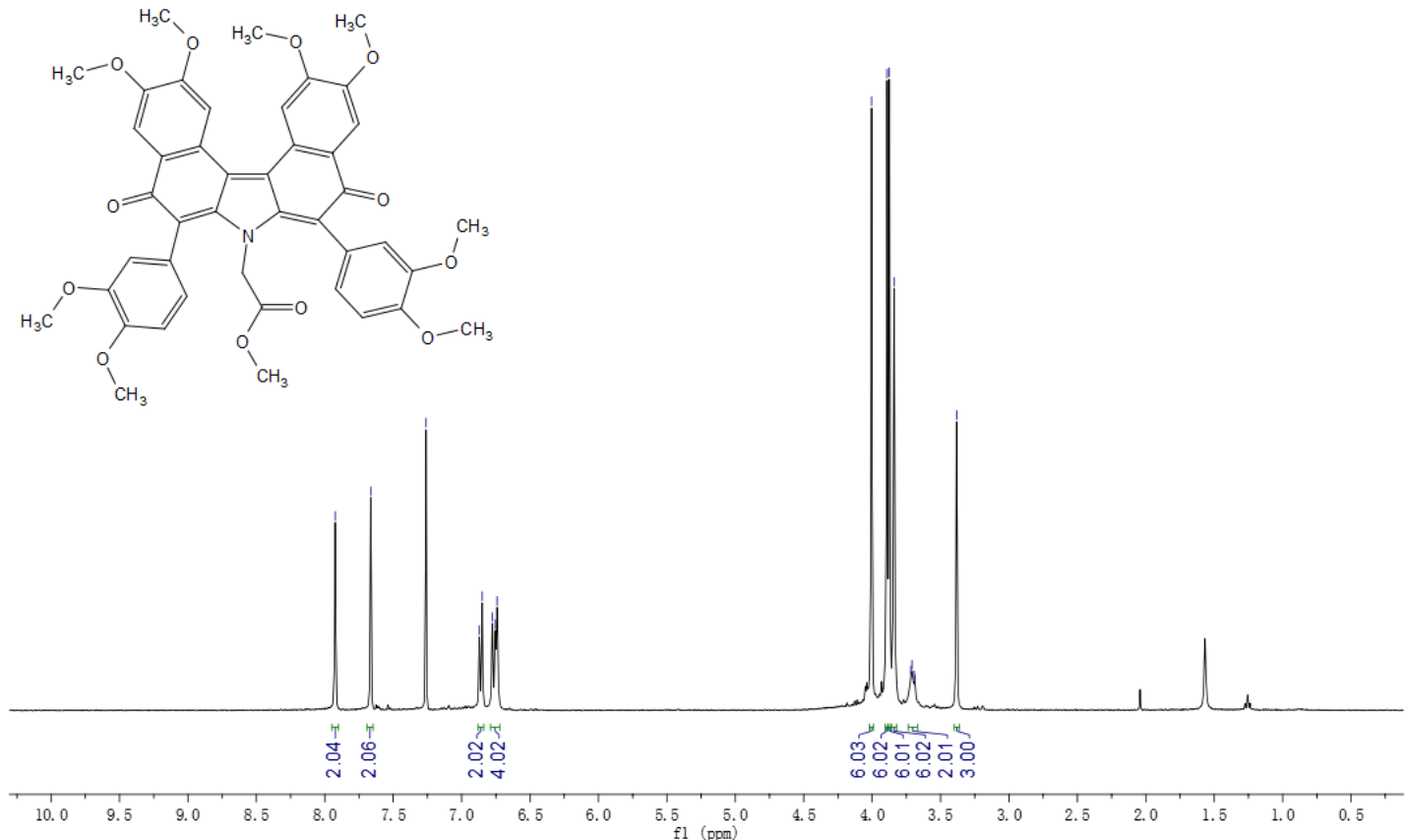

${ }^{13} \mathrm{C}$ NMR: II-135

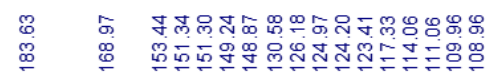

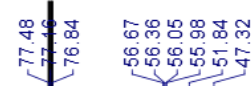

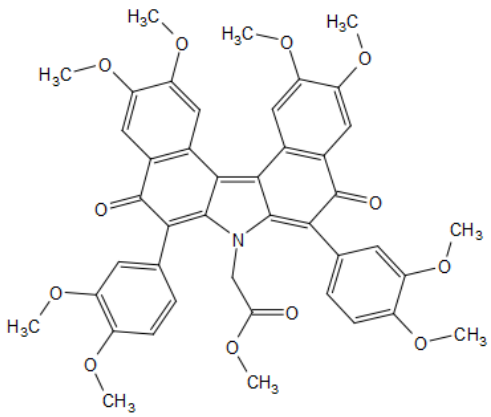

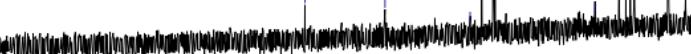

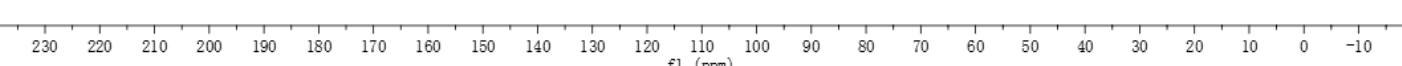


${ }^{1} \mathrm{H}$ NMR: 5

Solvent: $\mathrm{CDCl}_{3}$

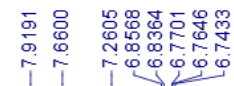

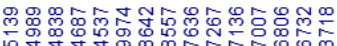

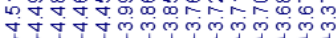

高尊
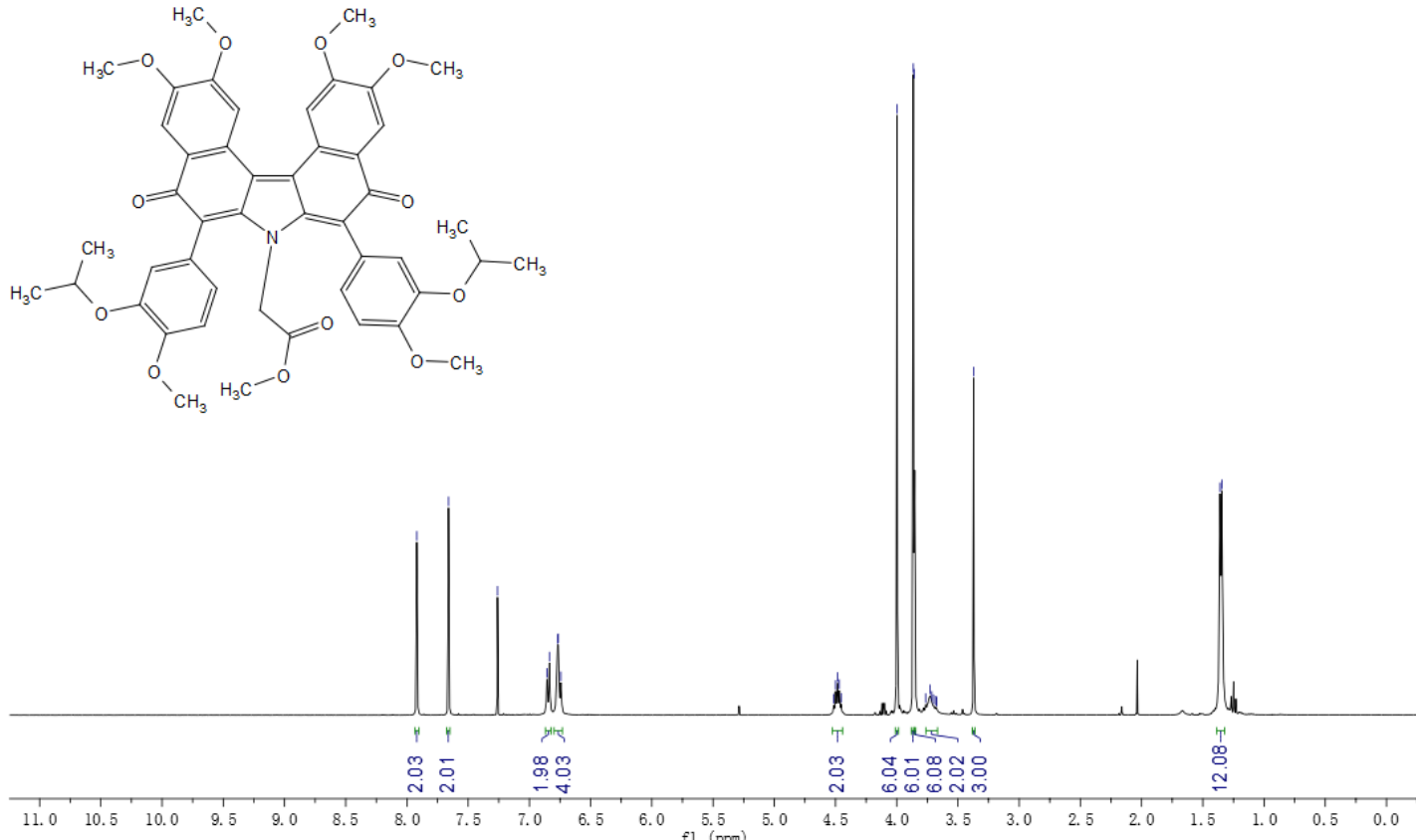

${ }^{13} \mathrm{C}$ NMR: 5

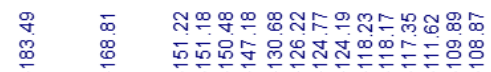

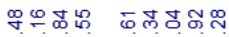

च-5E

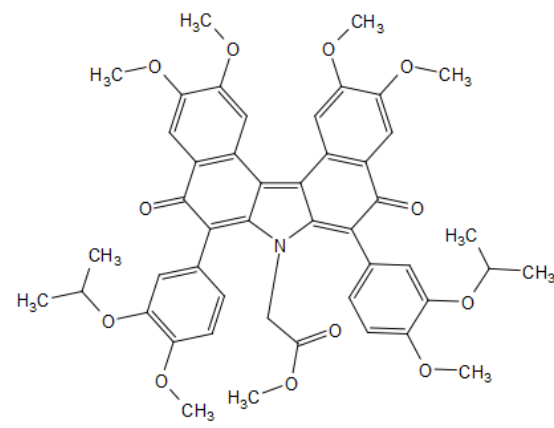

$\begin{array}{lllllllllllll}230 & 220 & 210 & 200 & 190 & 180 & 170 & 160 & 150 & 140 & 130 & 120 & 110 \\ \mathrm{fl} 1(\mathrm{prm}) & 100\end{array}$ 
${ }^{1} \mathrm{H}$ NMR: 13

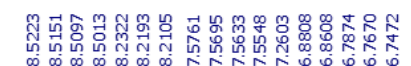

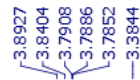
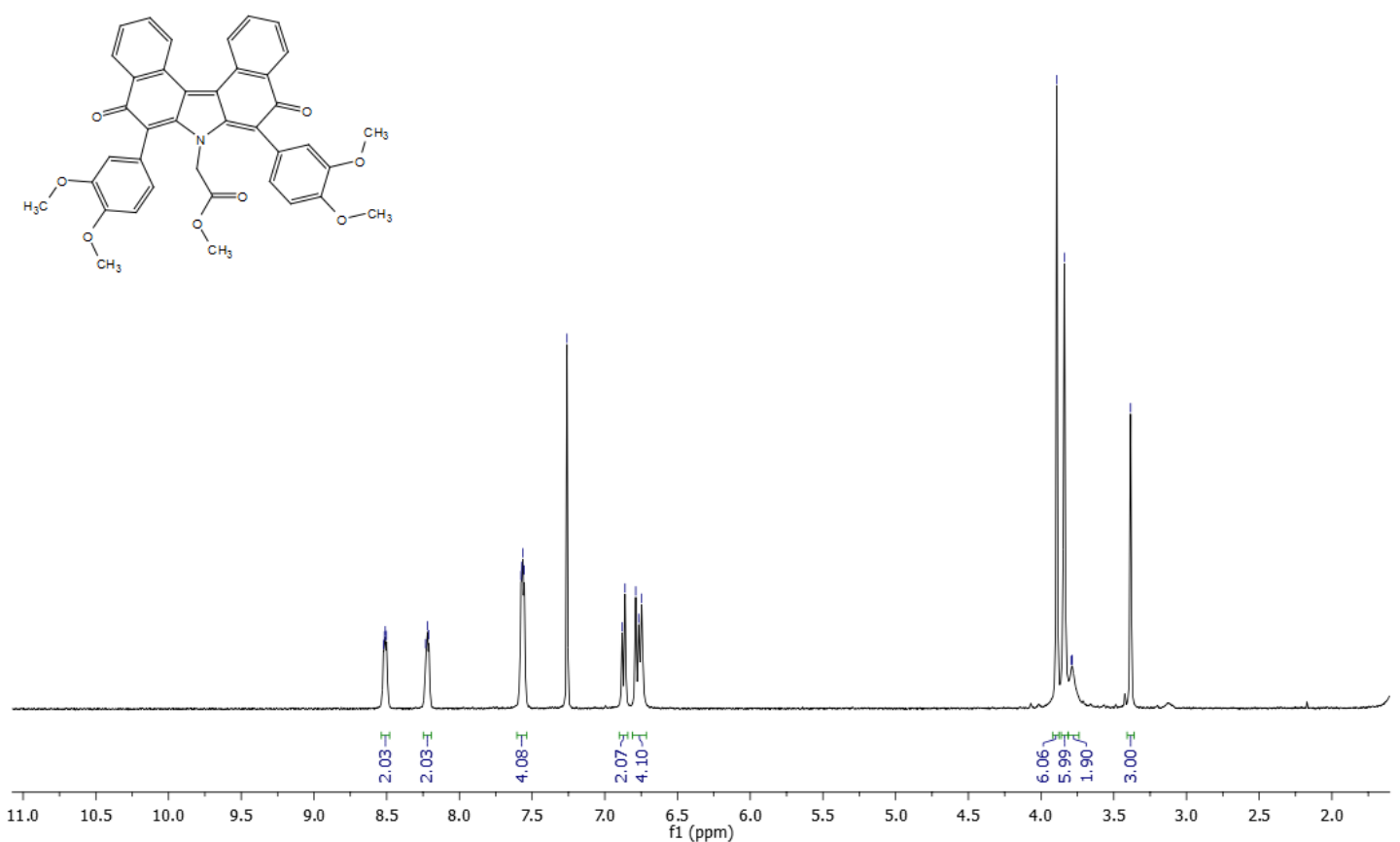

${ }^{13} \mathrm{C}$ NMR: 13

$m \quad$ क

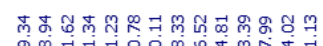

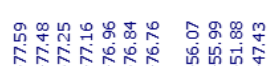

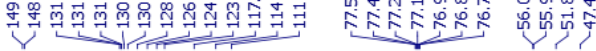
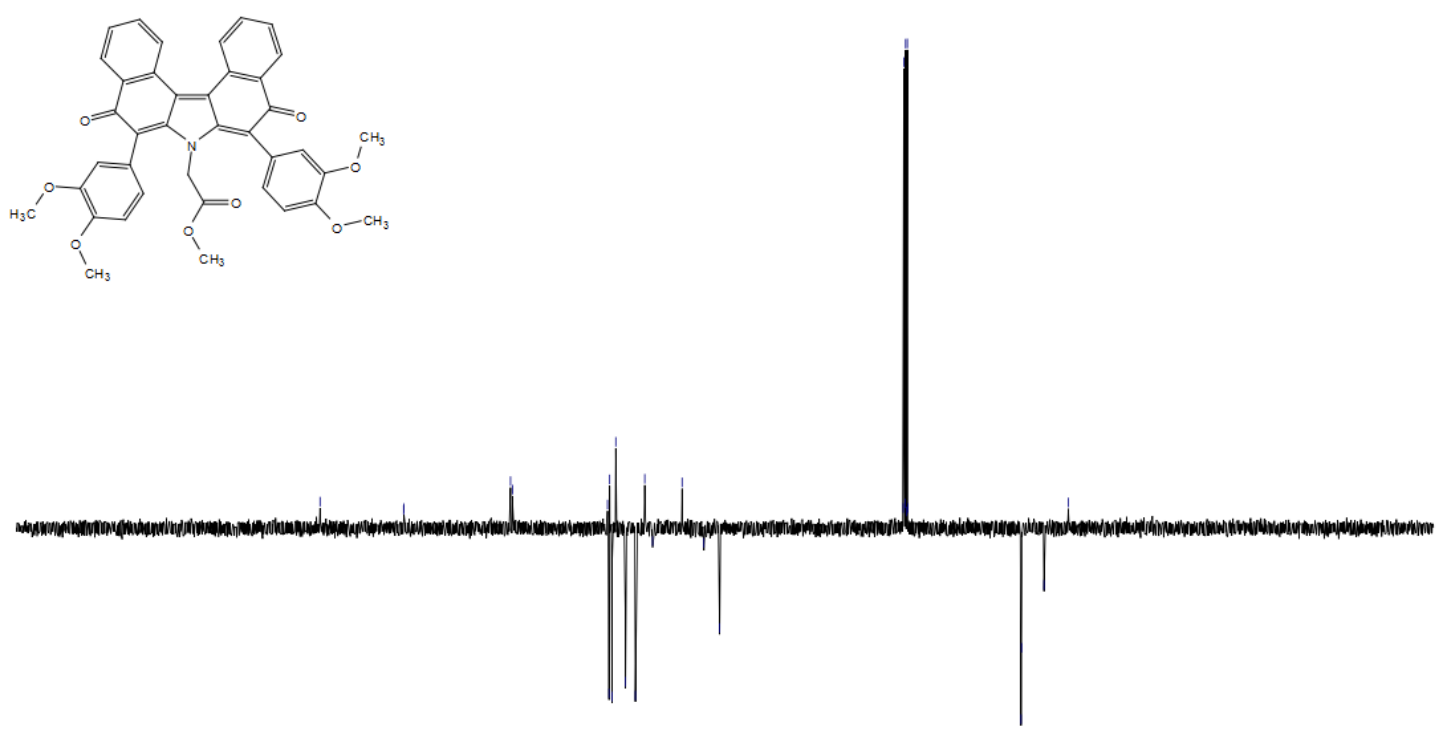

$\begin{array}{lllllllllllllllllllllllll}230 & 220 & 210 & 200 & 190 & 180 & 170 & 160 & 150 & 140 & 130 & 120 & 110 & 100 & 90 & 80 & 70 & 60 & 50 & 40 & 30 & 20 & 10 & 0 & -10\end{array}$ 


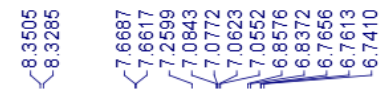

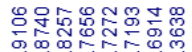

Solvent: $\mathrm{CDCl}_{3}$

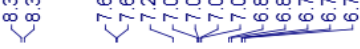

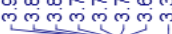
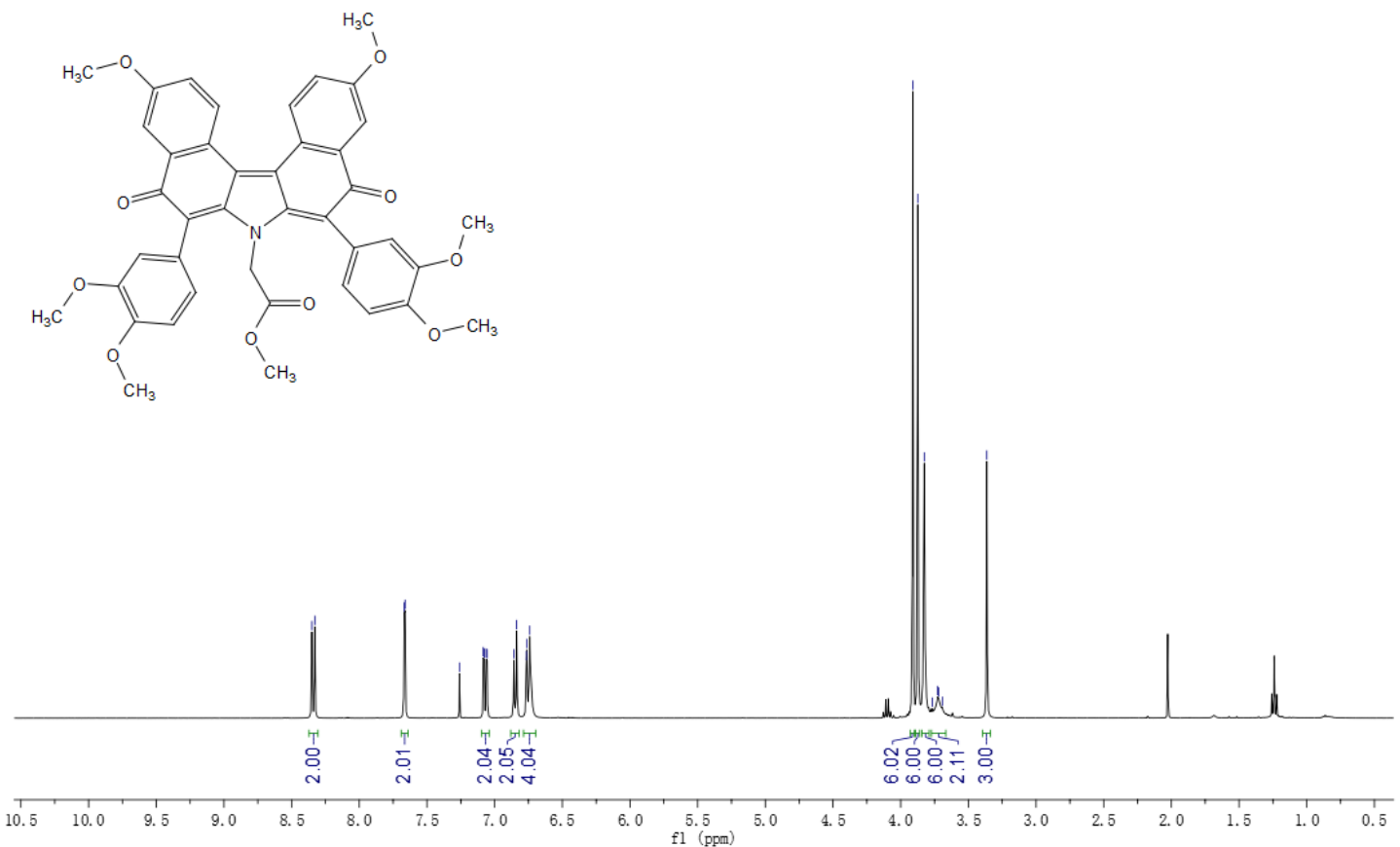

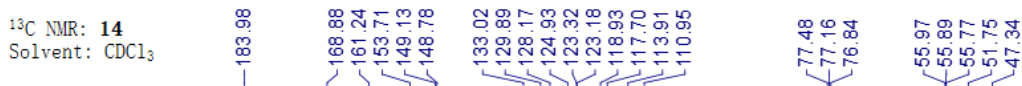
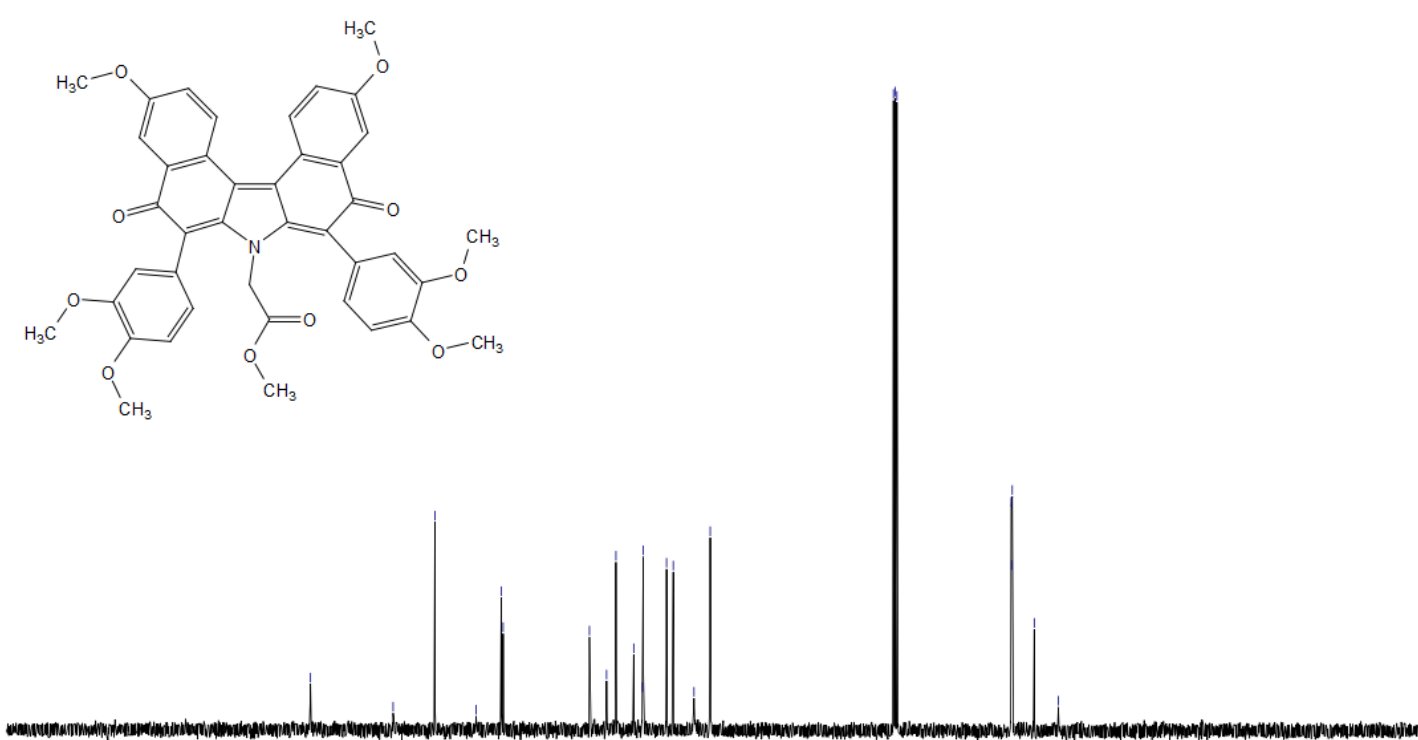

$\begin{array}{lllllllllllllllllllllllll}230 & 220 & 210 & 200 & 190 & 180 & 170 & 160 & 150 & 140 & 130 & 120 & 110 & 100 & 90 & 80 & 70 & 60 & 50 & 40 & 30 & 20 & 10 & 0 & -10\end{array}$ 


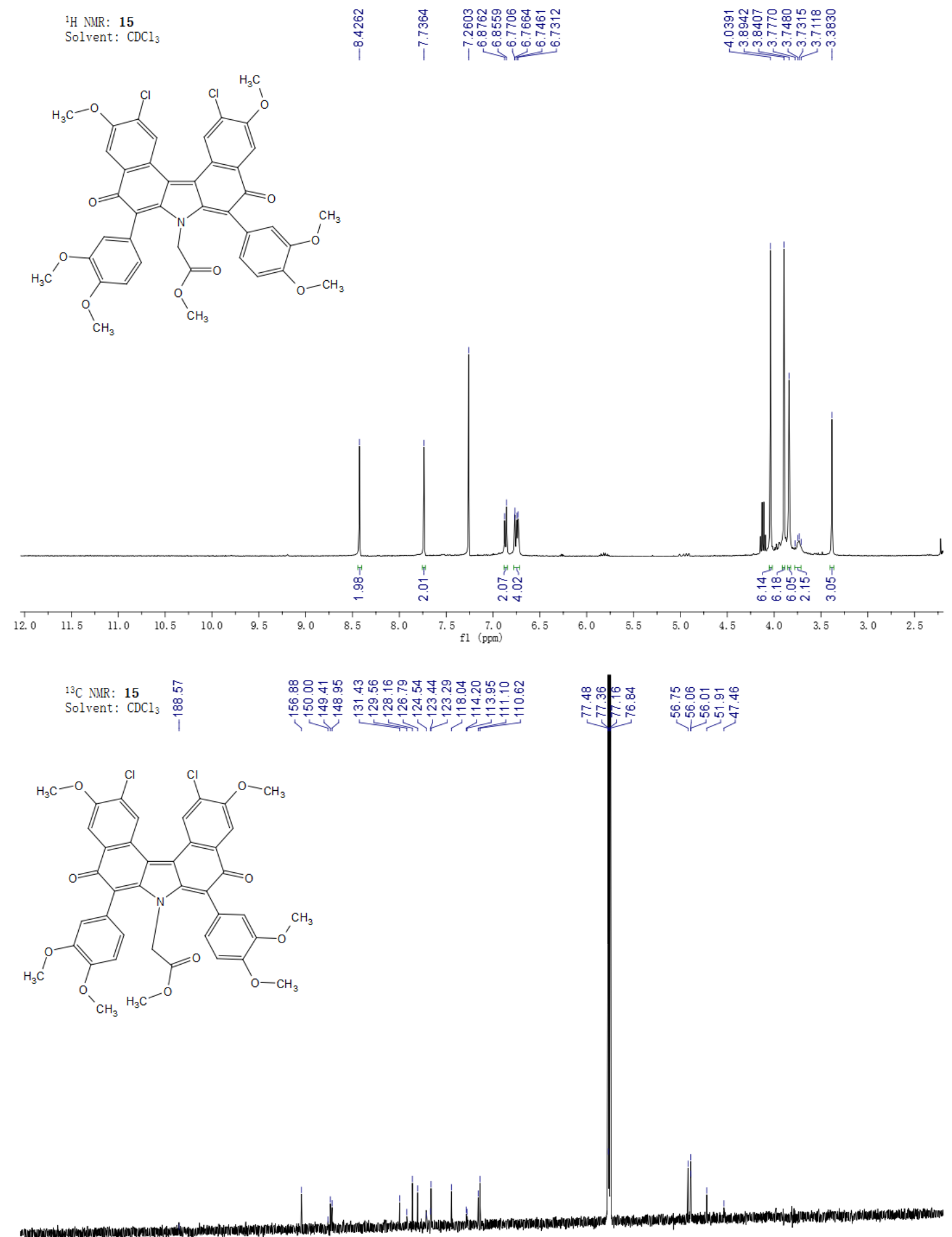

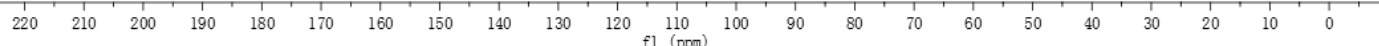




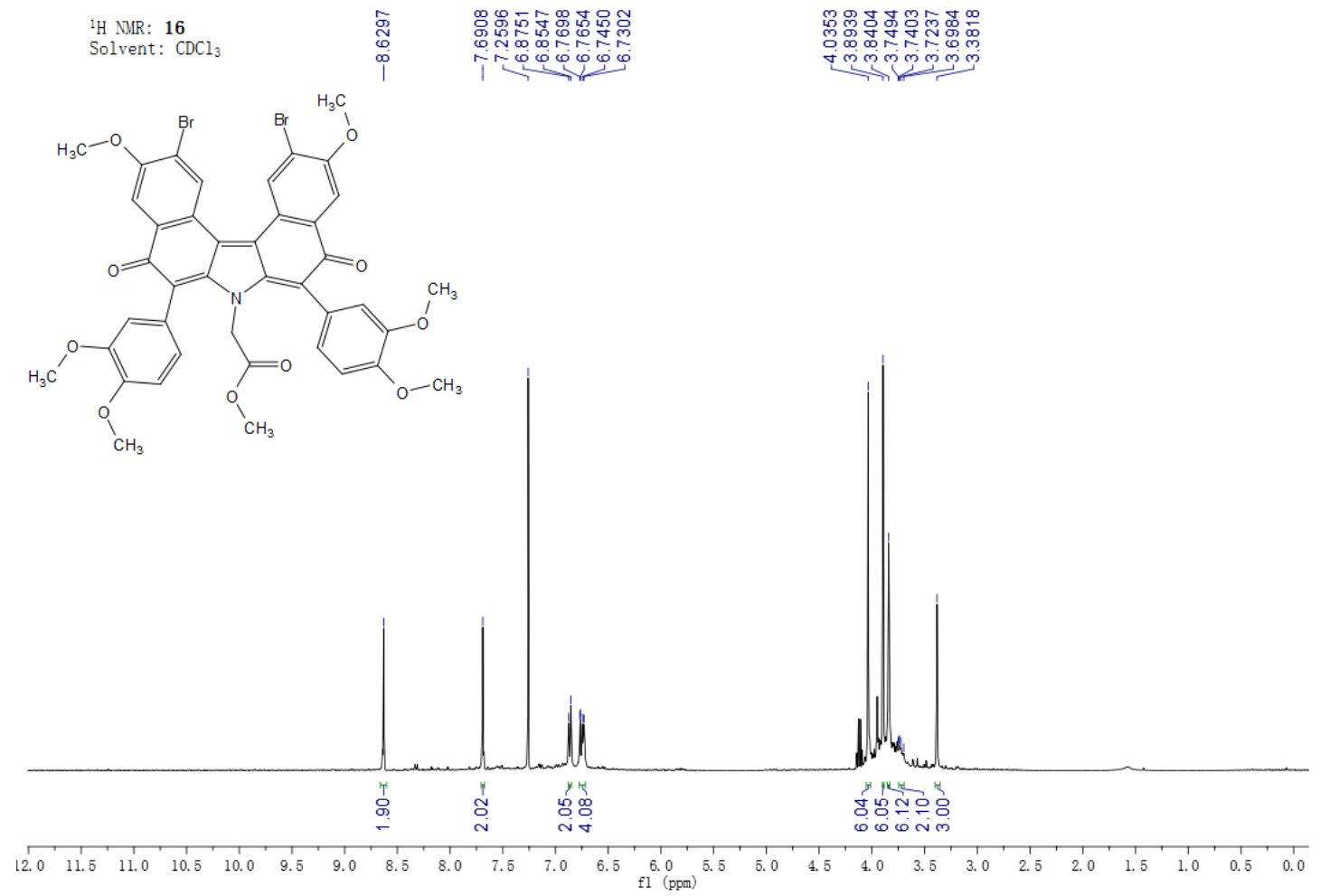

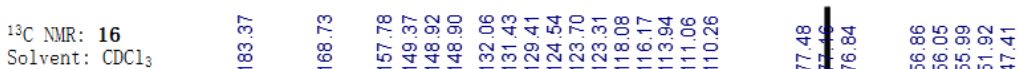

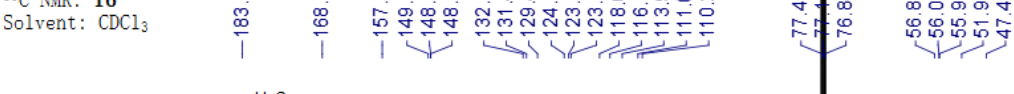

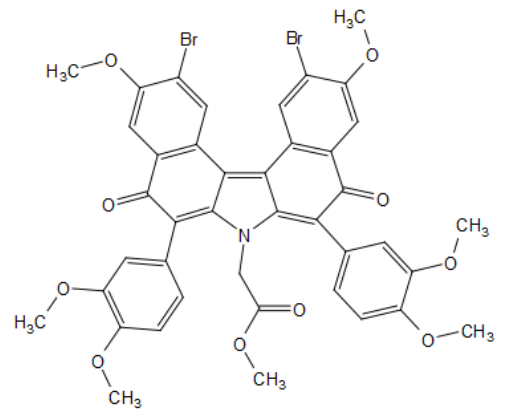

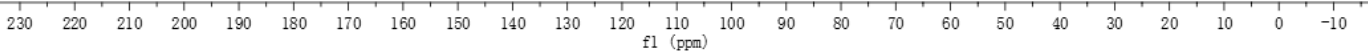




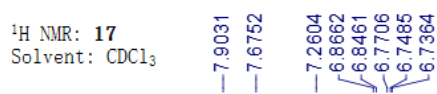

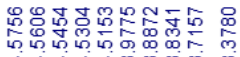
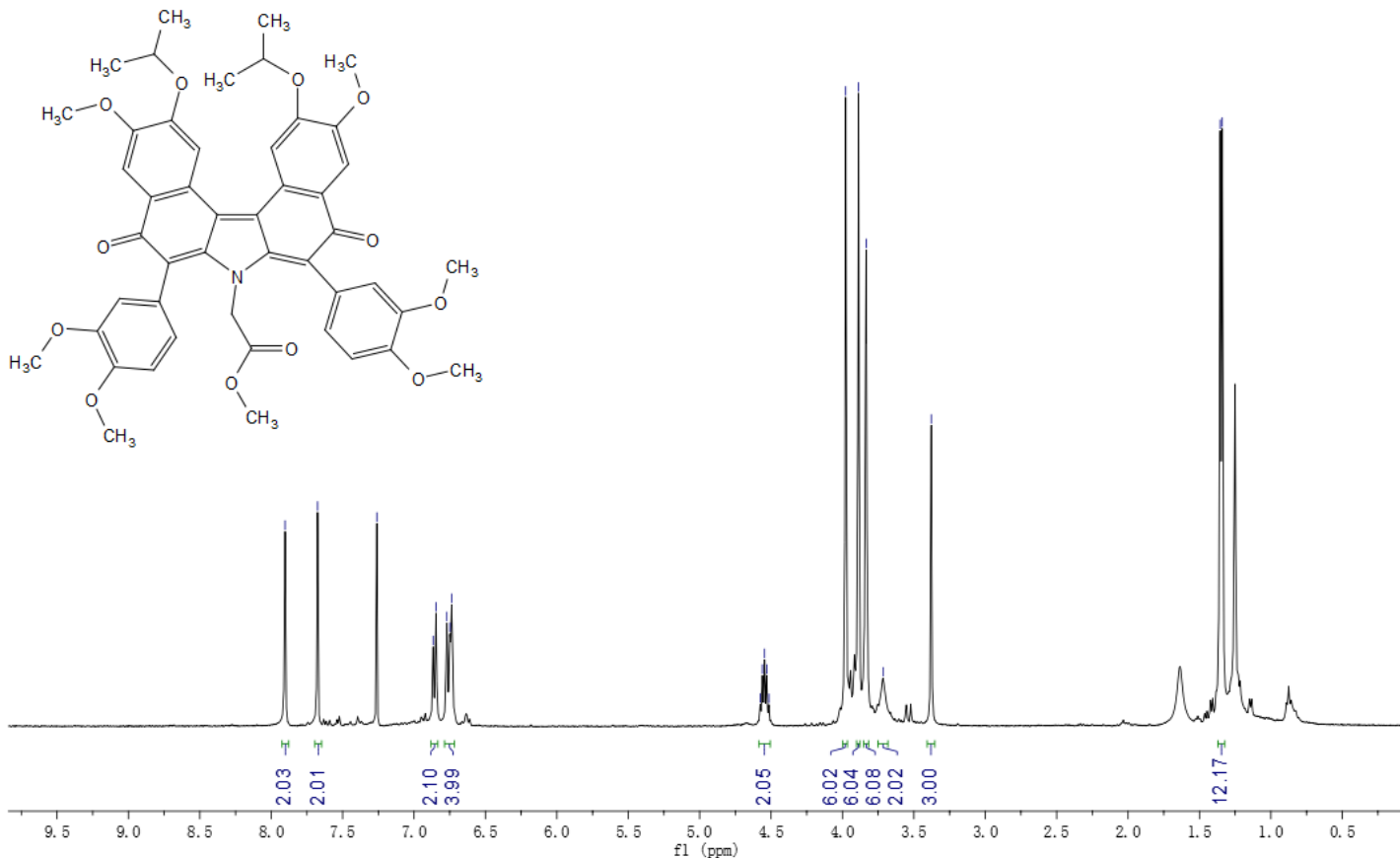

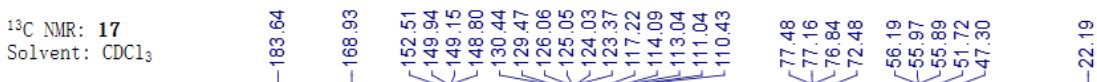

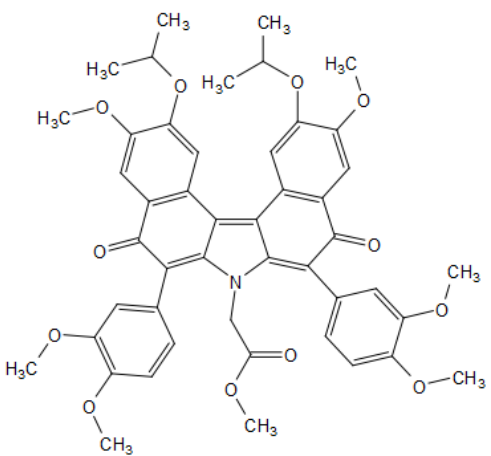

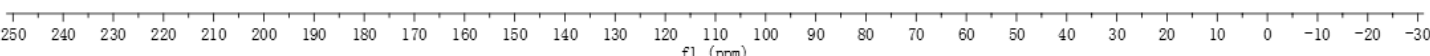

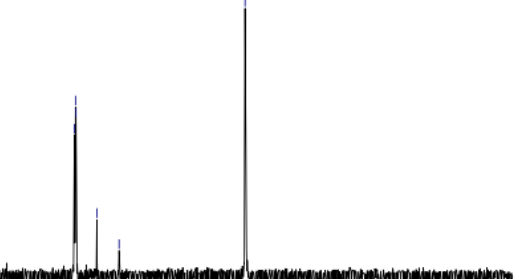


${ }^{1} \mathrm{H}$ NMR: 6

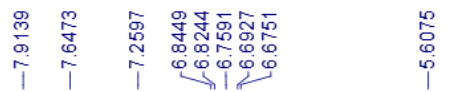

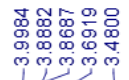
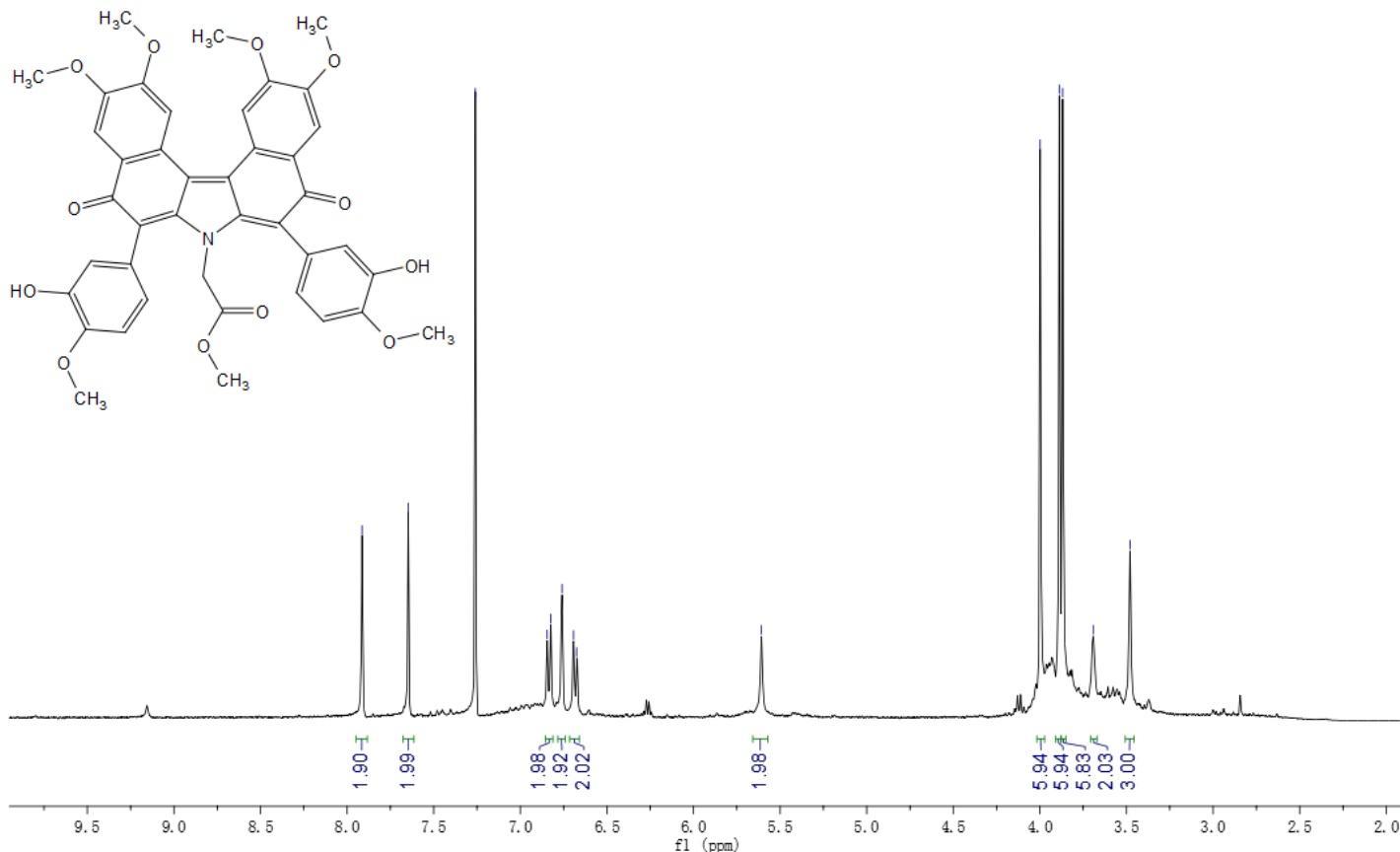

${ }^{13} \mathrm{C}$ NMR: 6

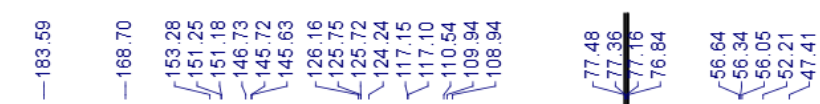
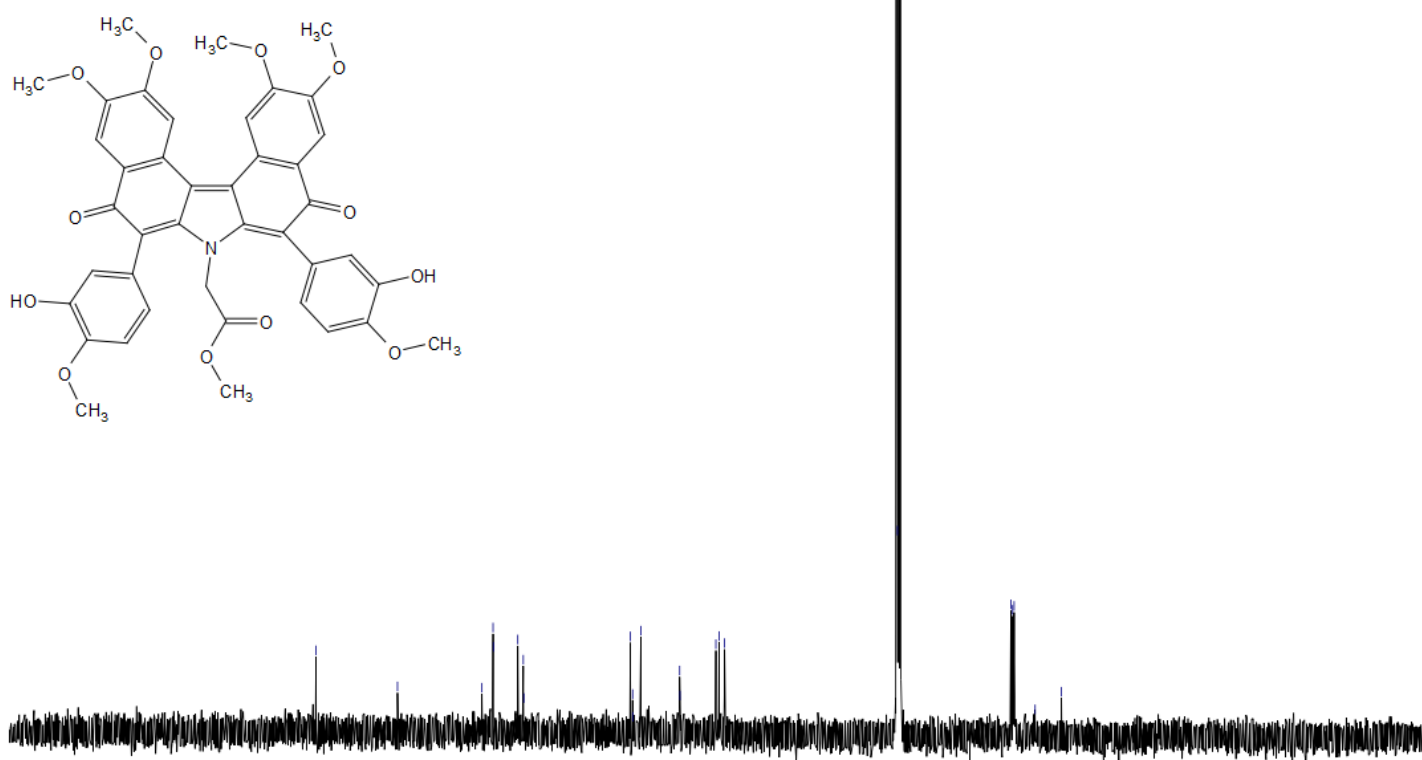

$\begin{array}{lllllllllllllllllllllllllllllllllllll}230 & 220 & 210 & 200 & 190 & 180 & 170 & 160 & 150 & 140 & 130 & 120 & 110 & 100 & 90 & 80 & 70 & 60 & 50 & 40 & 30 & 20 & 10 & 0 & -10\end{array}$ 
${ }^{1} \mathrm{H}$ NMR: 18
Solvent: $\mathrm{CDCl}$

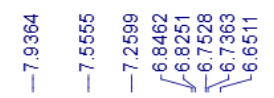

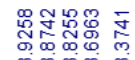
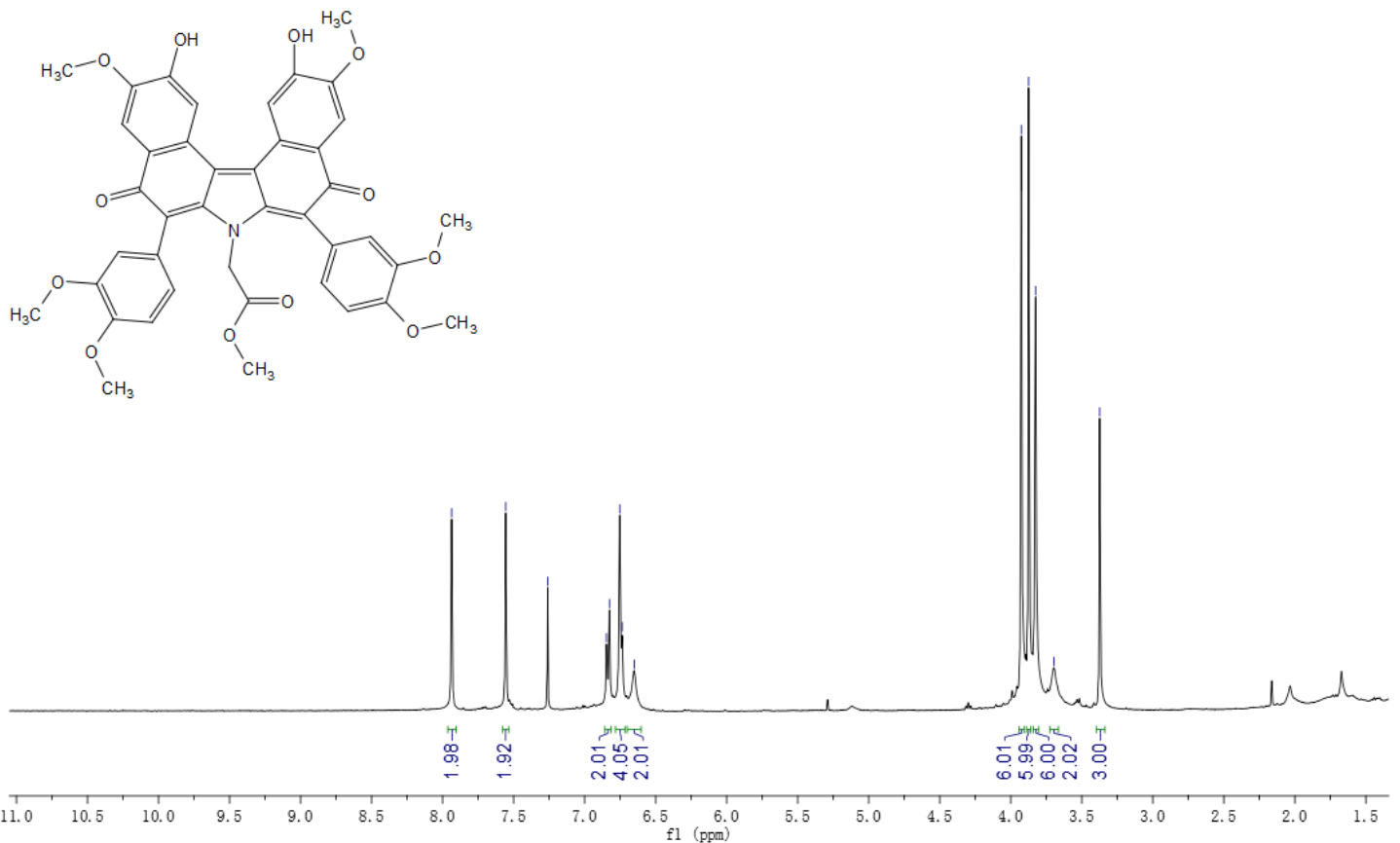

${ }^{13} \mathrm{C}$ NMR: 18

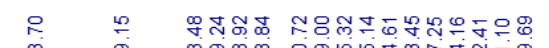

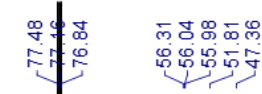

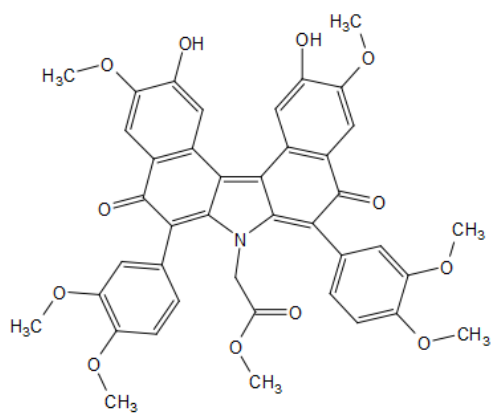

$\mid$

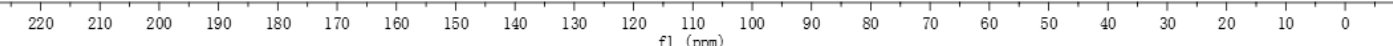




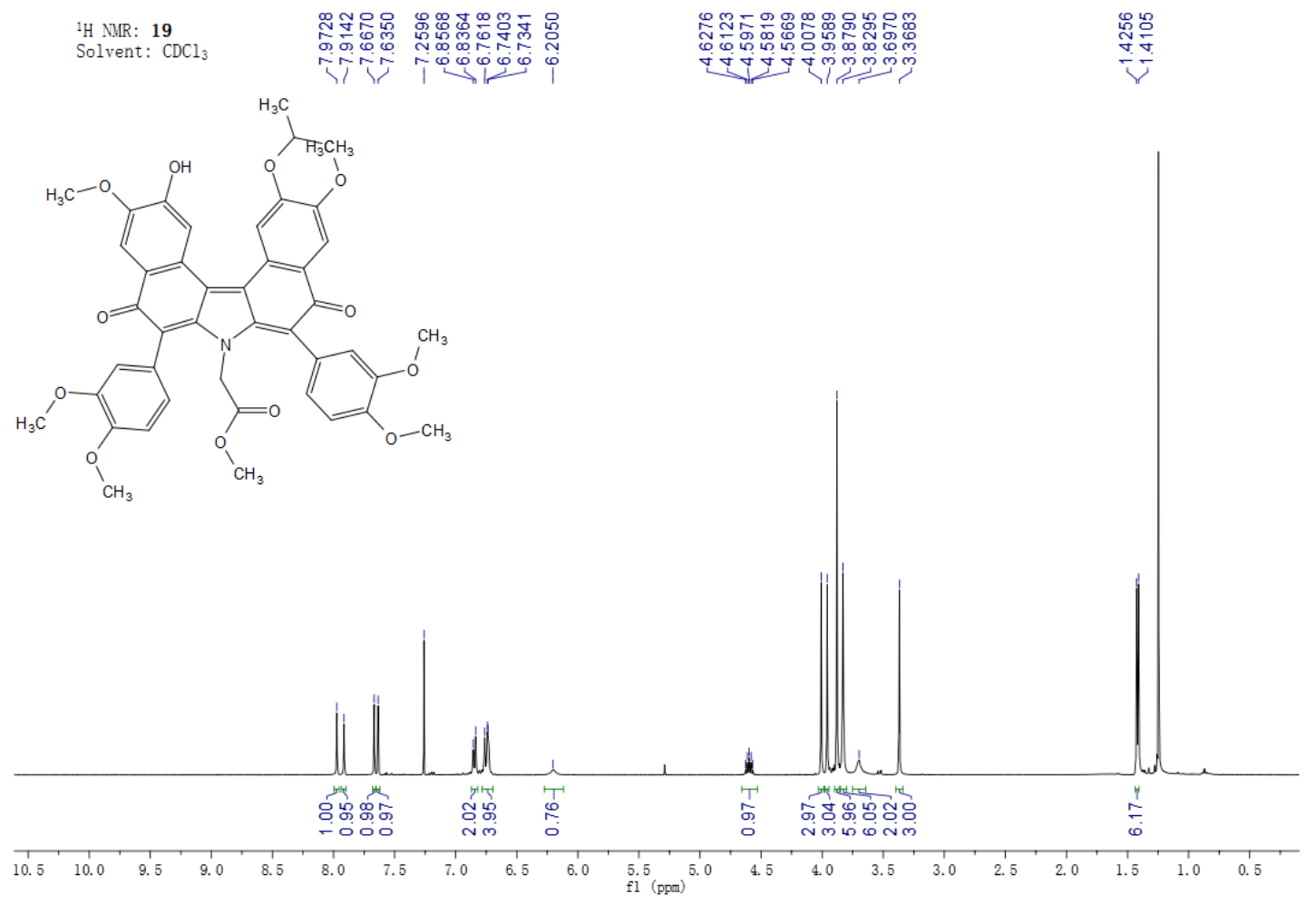

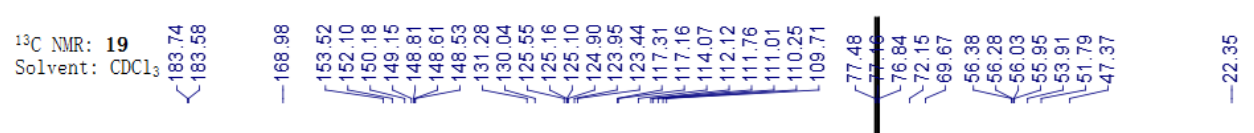
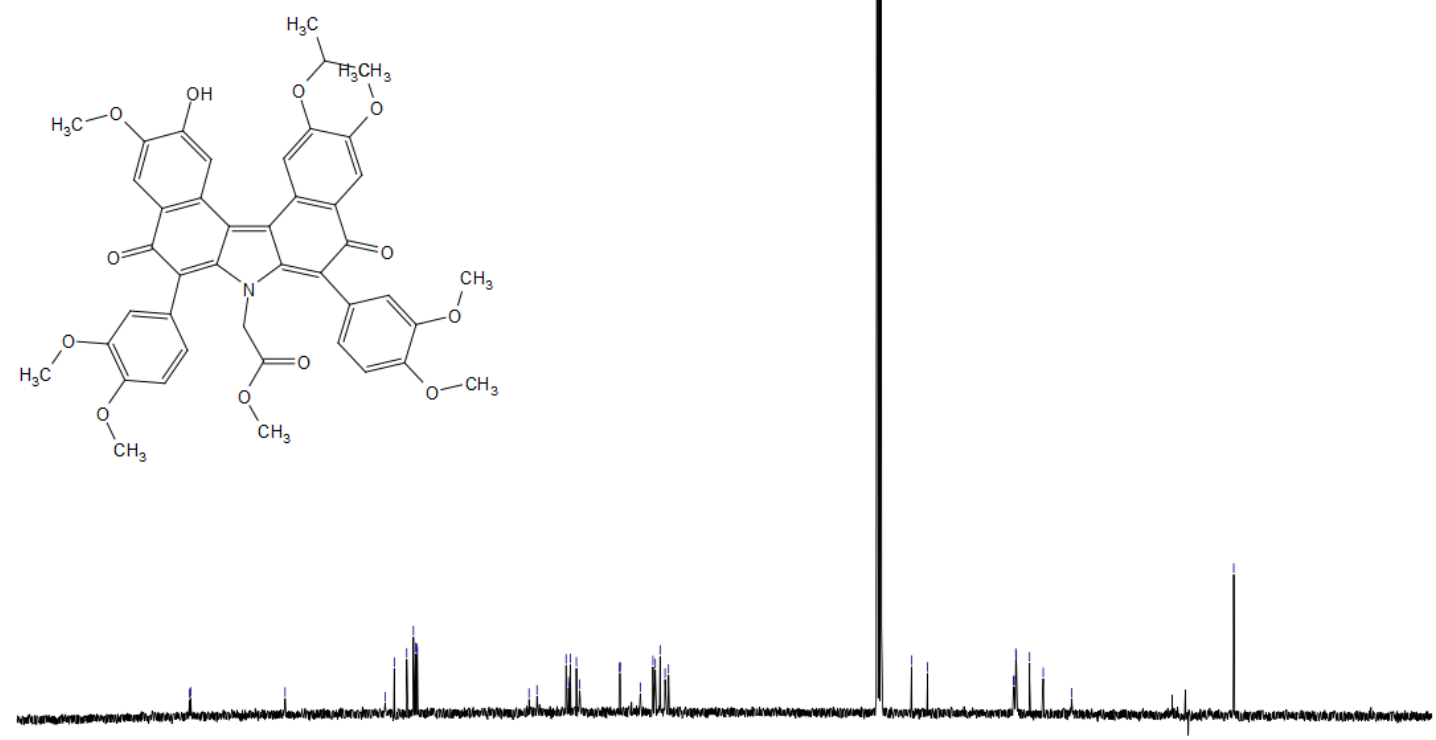

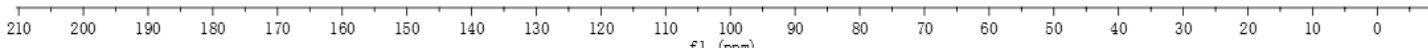


${ }^{1} \mathrm{H}$ NMR: 8

Solvent: $\mathrm{CDCl}_{3}$

学 总

i

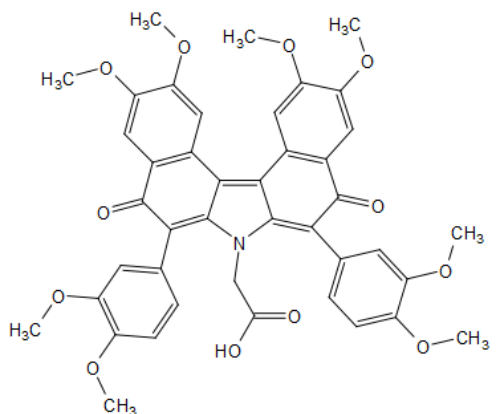

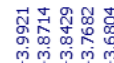

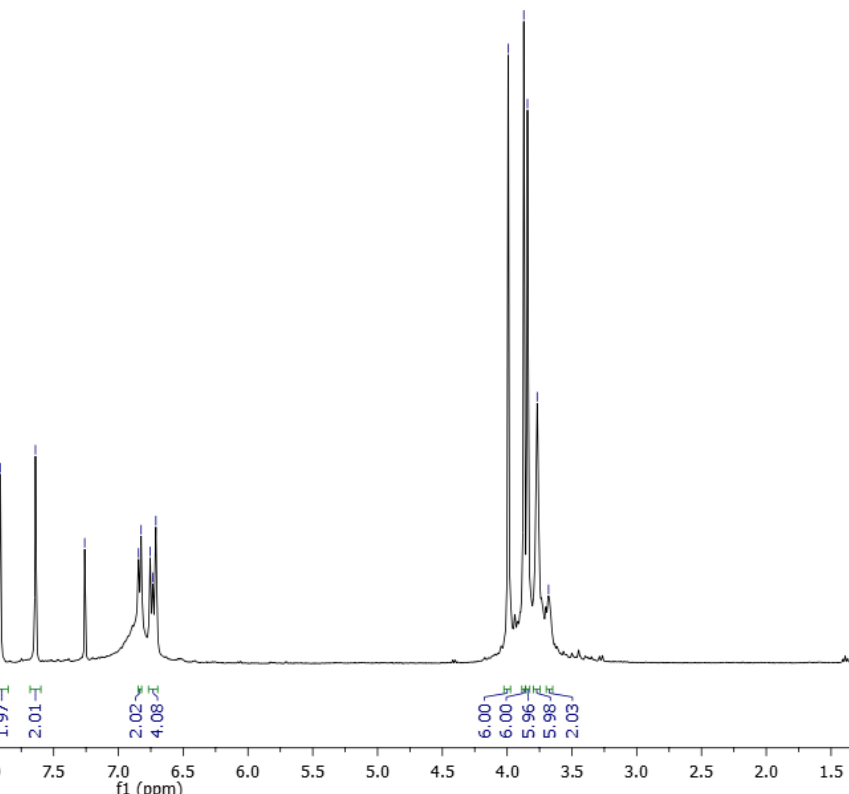

${ }^{13} \mathrm{C}$ NUR: 8

Solvent: $\mathrm{CDCl}_{3} \quad \stackrel{+}{\stackrel{\infty}{\perp}}$

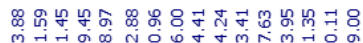

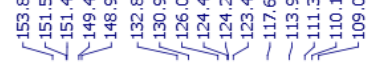

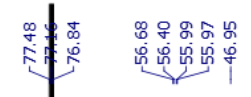
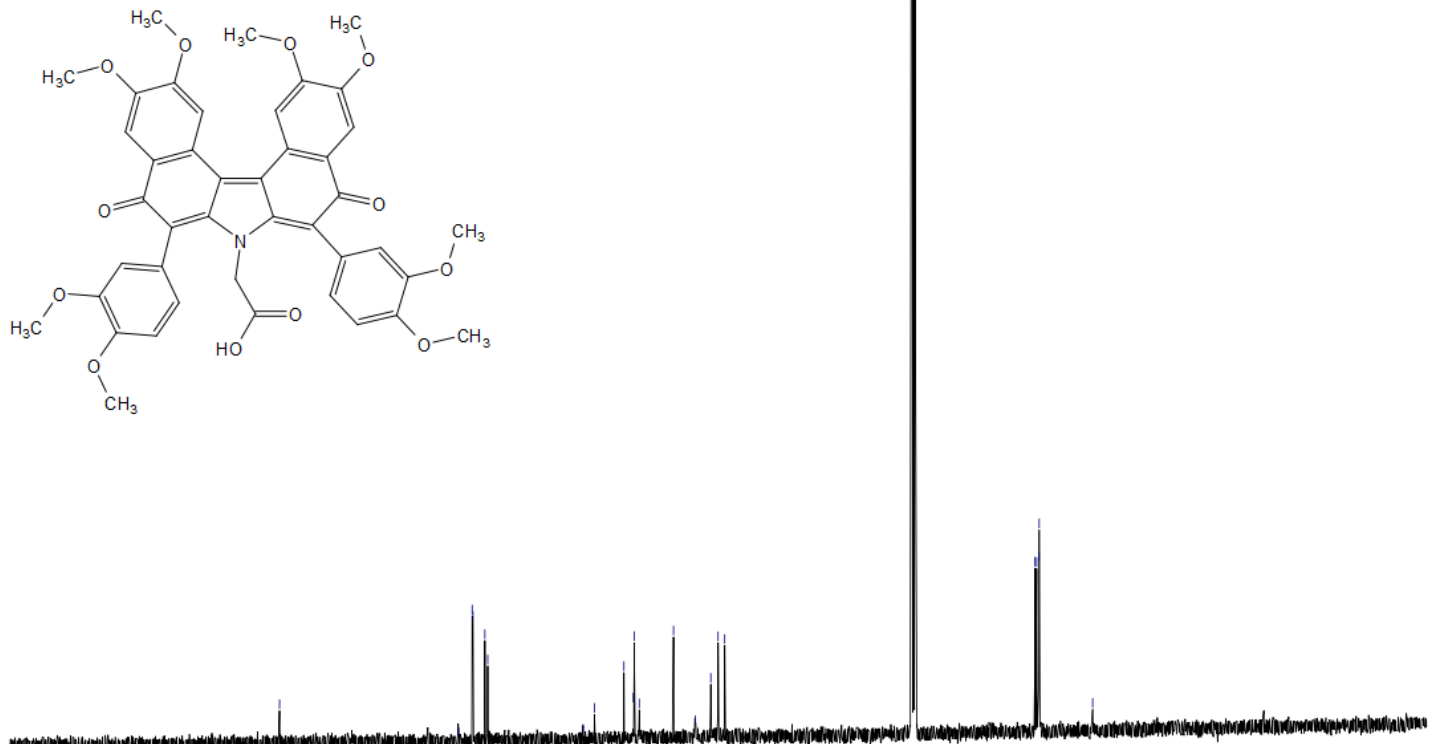

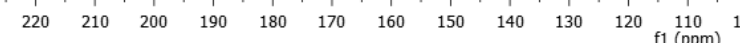



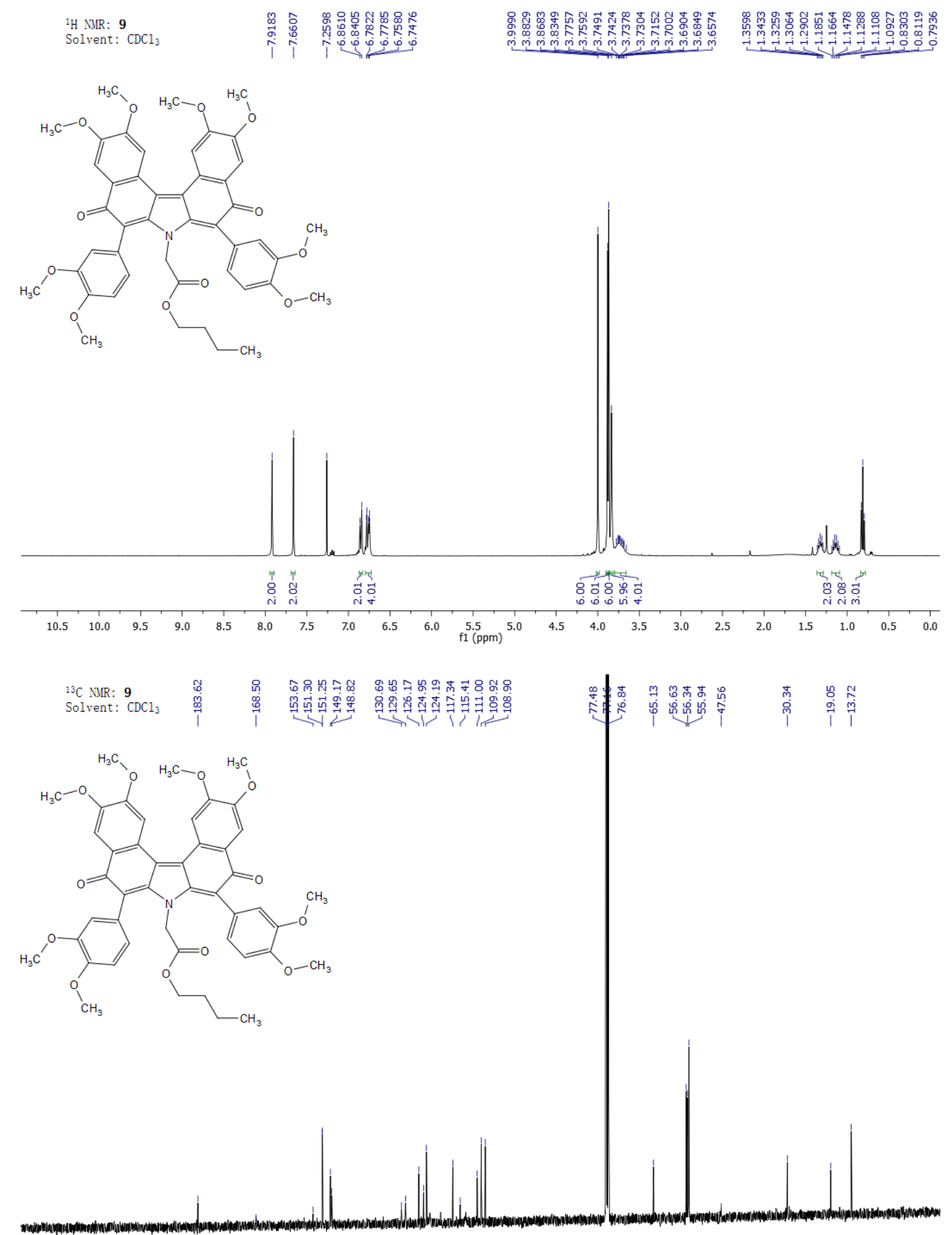

$\begin{array}{lllllllllllllllllllllllll}220 & 210 & 200 & 190 & 180 & 170 & 160 & 150 & 140 & 130 & 120 & 110 & 100 & 90 & 80 & 70 & 60 & 50 & 40 & 30 & 20 & 10 & 0 & \end{array}$ 


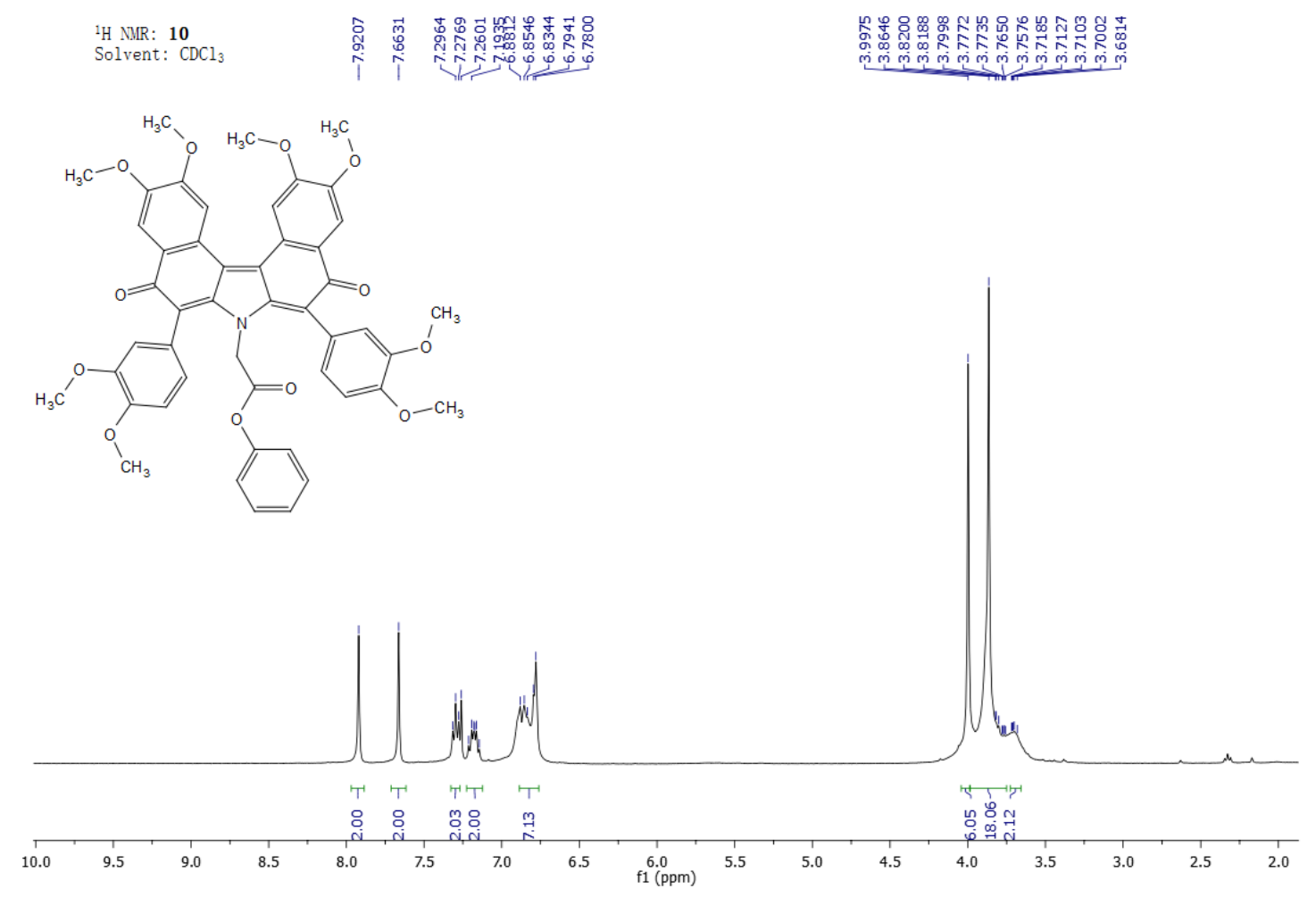

$\begin{array}{lll}{ }^{13} \mathrm{CNMR}: 10 & 0 & 0 \\ \text { Solvent: } \mathrm{CDCl}_{3} & 0 & 0\end{array}$
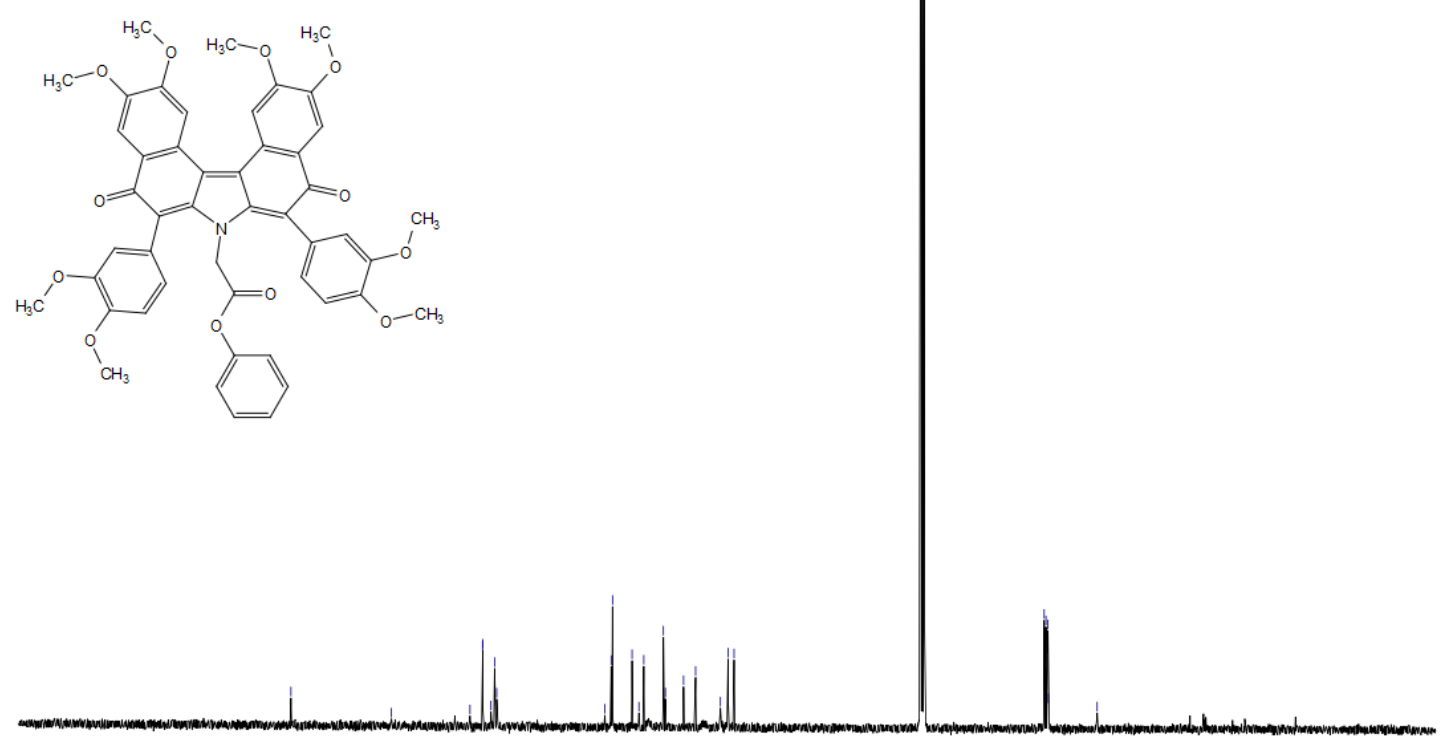

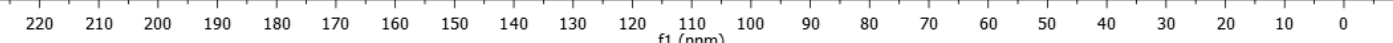


${ }^{1} \mathrm{H}$ NMR: 11
Solvent: $\mathrm{CDCl}$ ?

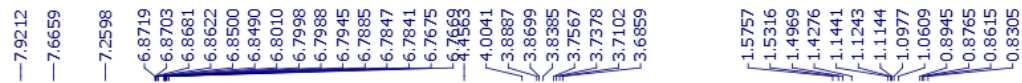
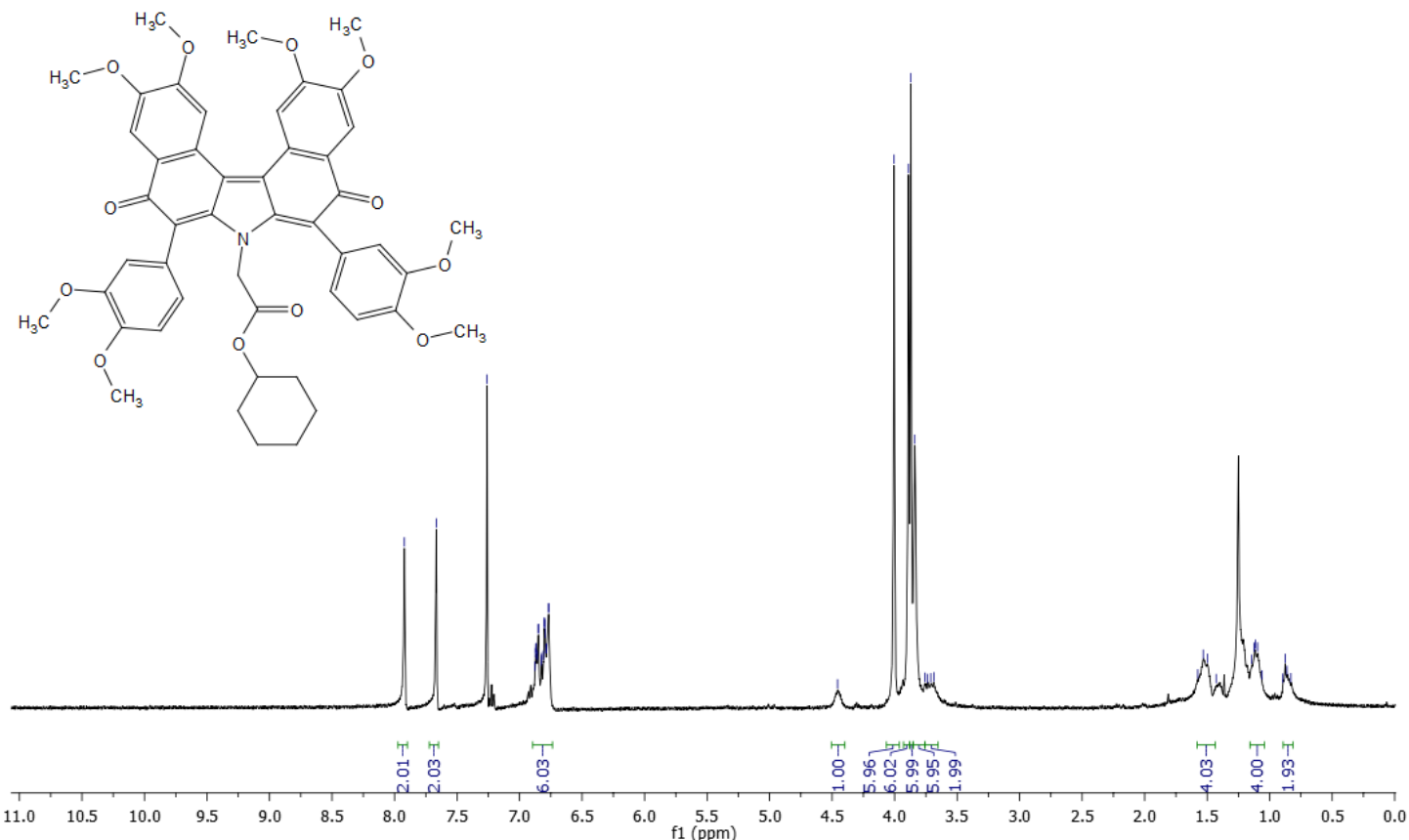

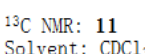

m.

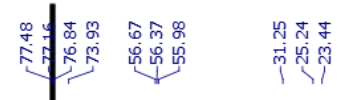

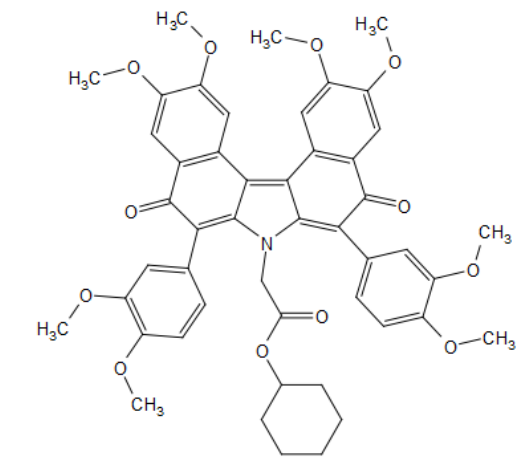

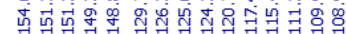

$\begin{array}{lllllllllllllllllllllllllllllllllllllll}250 & 240 & 230 & 220 & 210 & 200 & 190 & 180 & 170 & 160 & 150 & 140 & 130 & 120 & 110 & 100 & 90 & 80 & 70 & 60 & 50 & 40 & 30 & 20 & 10 & 0 & -10 & -20 & -30\end{array}$ 

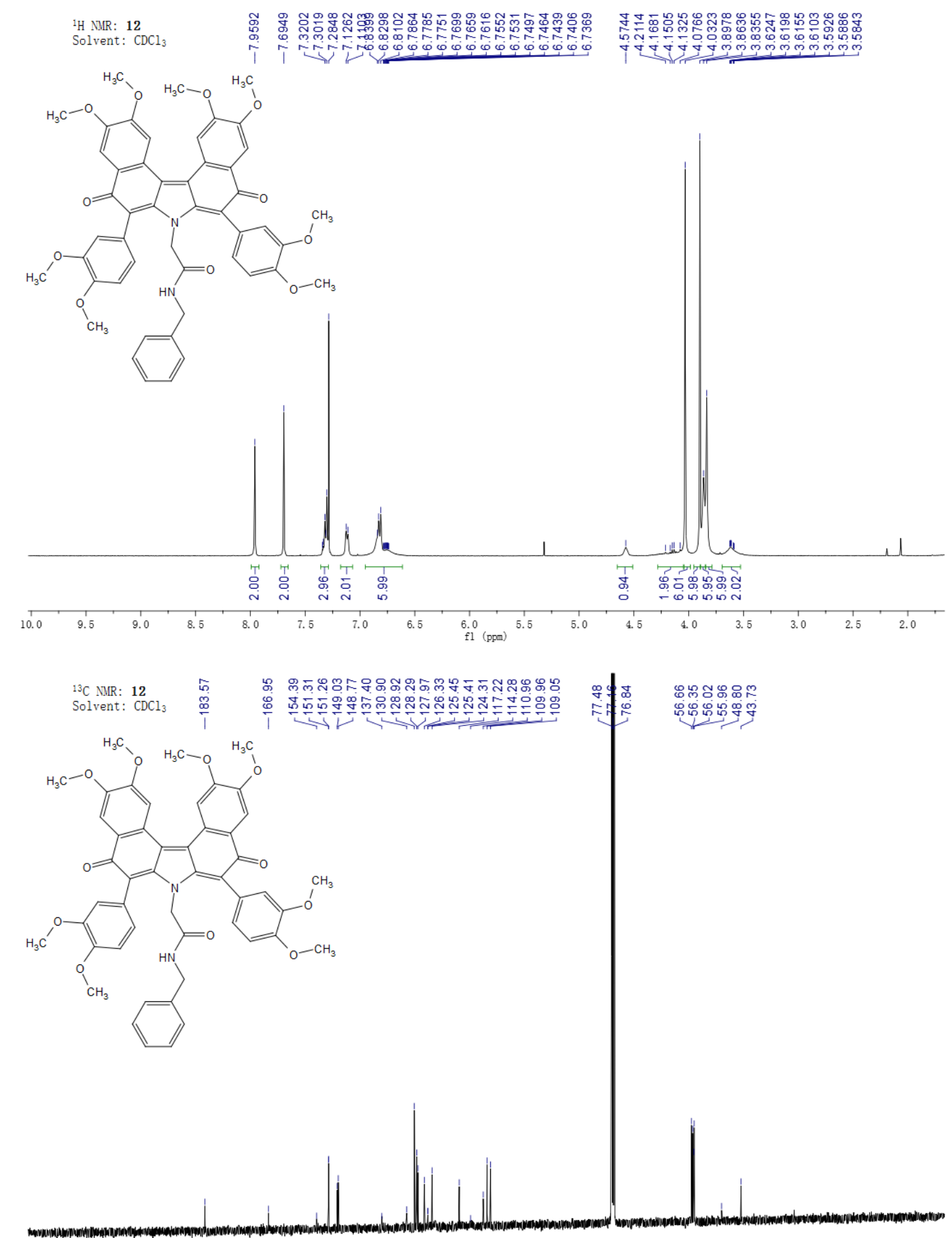

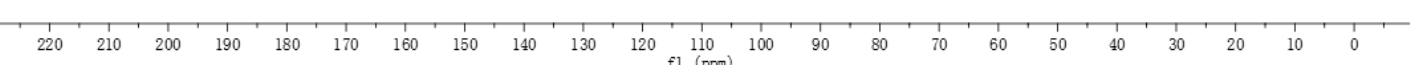




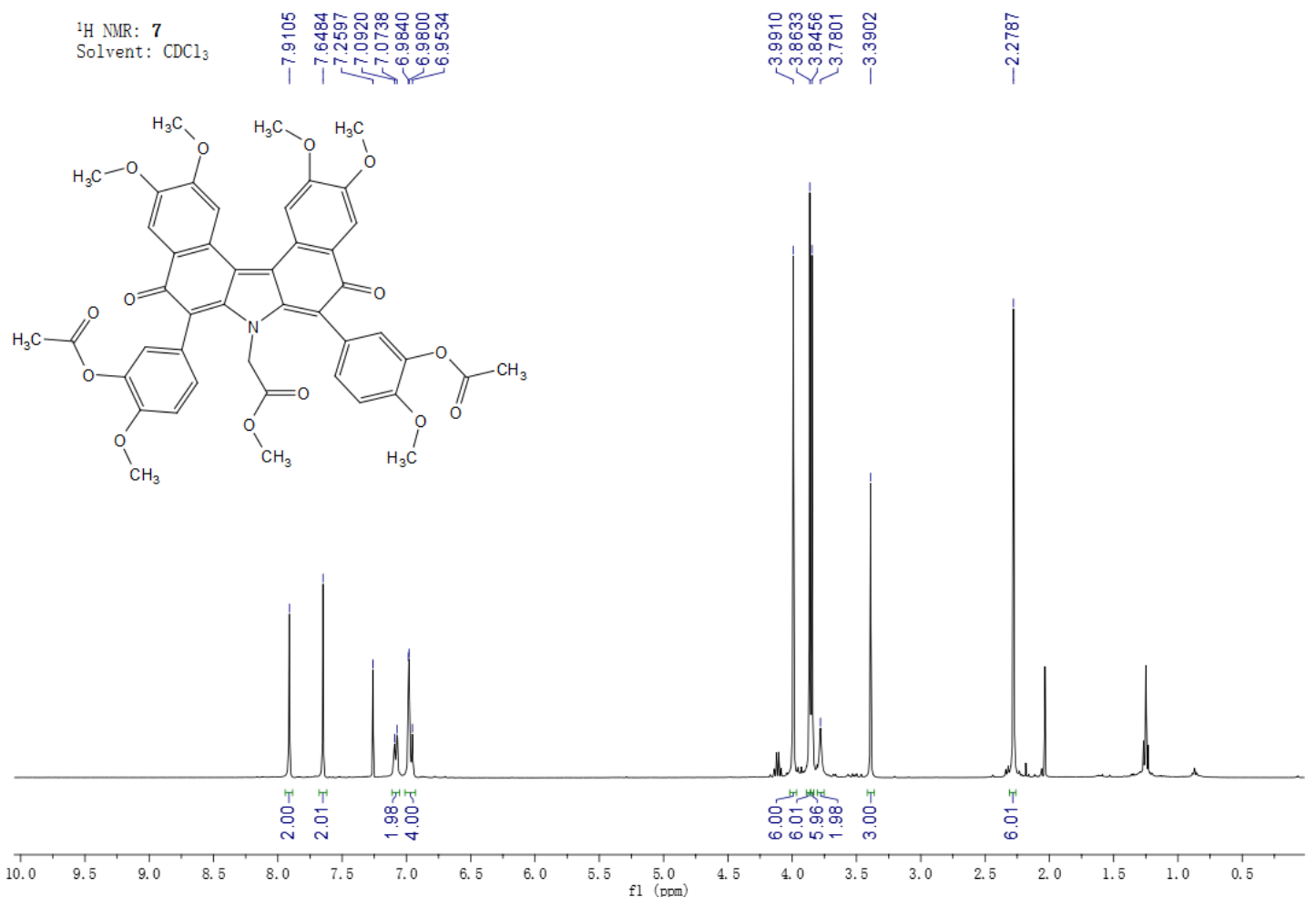

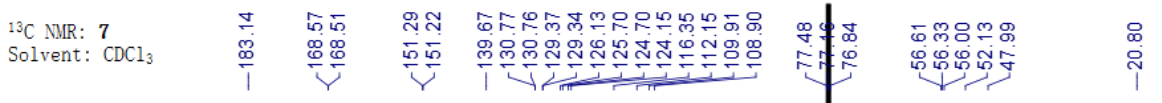
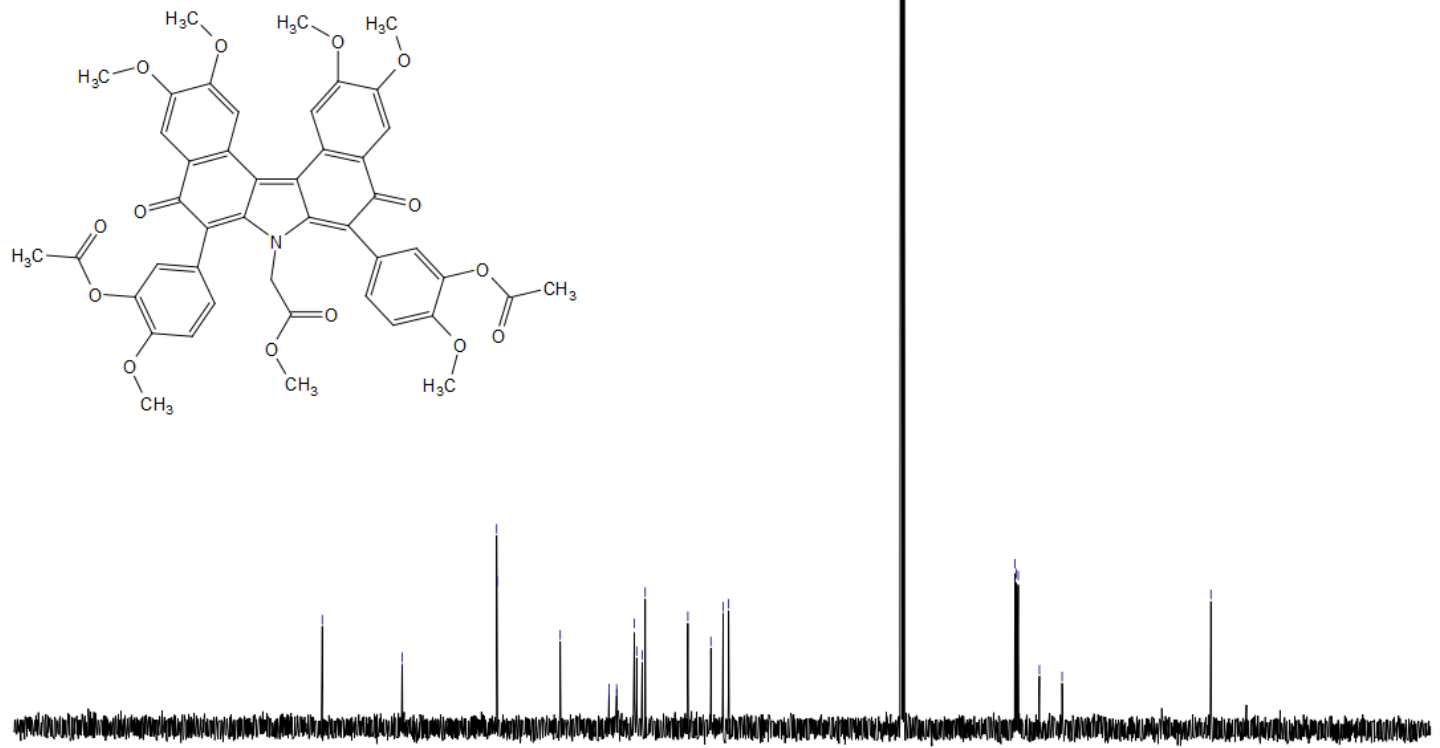

$\begin{array}{lllllllllllllllllllllllllllll}230 & 220 & 210 & 200 & 190 & 180 & 170 & 160 & 150 & 140 & 130 & 120 & 110 & 100 & 90 & 80 & 70 & 60 & 50 & 40 & 30 & 20 & 10 & 0 & -10\end{array}$ 

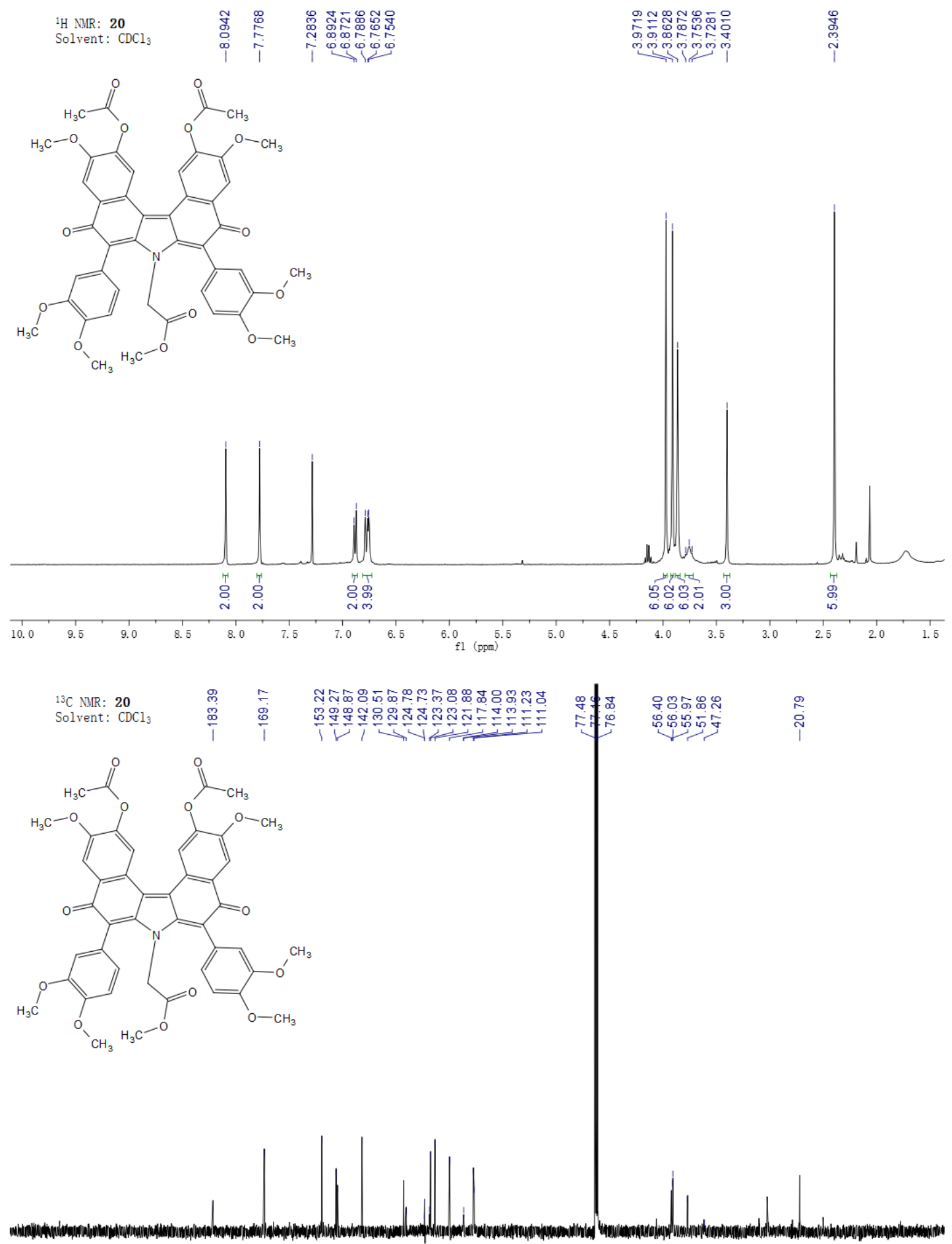

$\begin{array}{lllllllllllllllllllllllllllllll}230 & 220 & 210 & 200 & 190 & 180 & 170 & 160 & 150 & 140 & 130 & 120 & 110 & 100 & 90 & 80 & 70 & 60 & 50 & 40 & 30 & 20 & 10 & 0 & -10\end{array}$ 
${ }^{1} \mathrm{H}$ NMR: 21

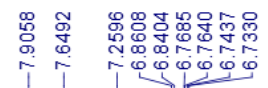

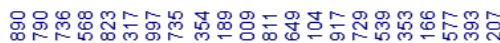

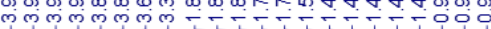
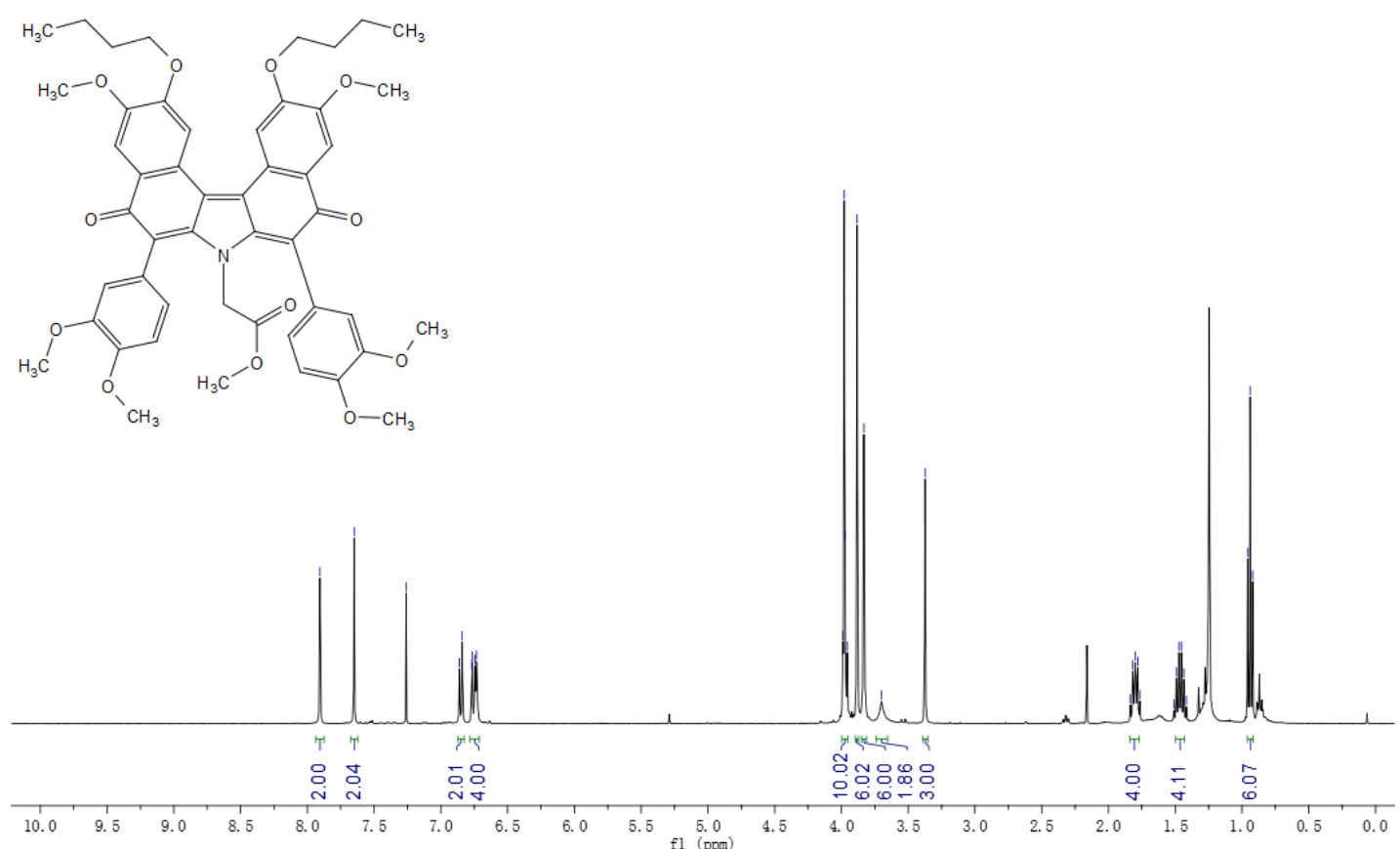

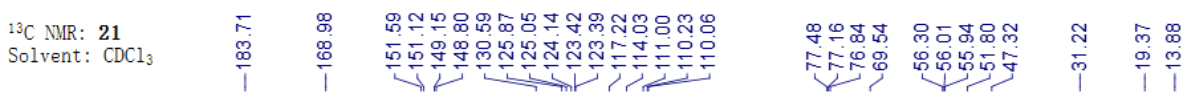

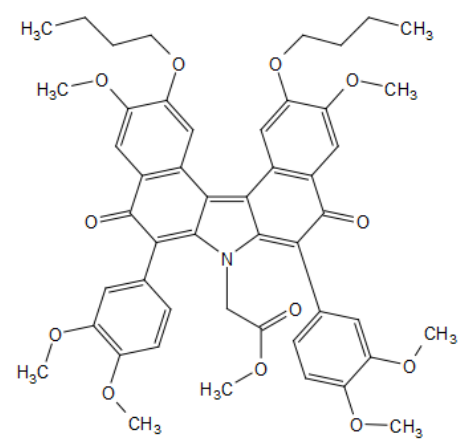

$\begin{array}{lllllllllllll}230 & 220 & 210 & 200 & 190 & 180 & 170 & 160 & 150 & 140 & 130 & 120 & 110\end{array}$
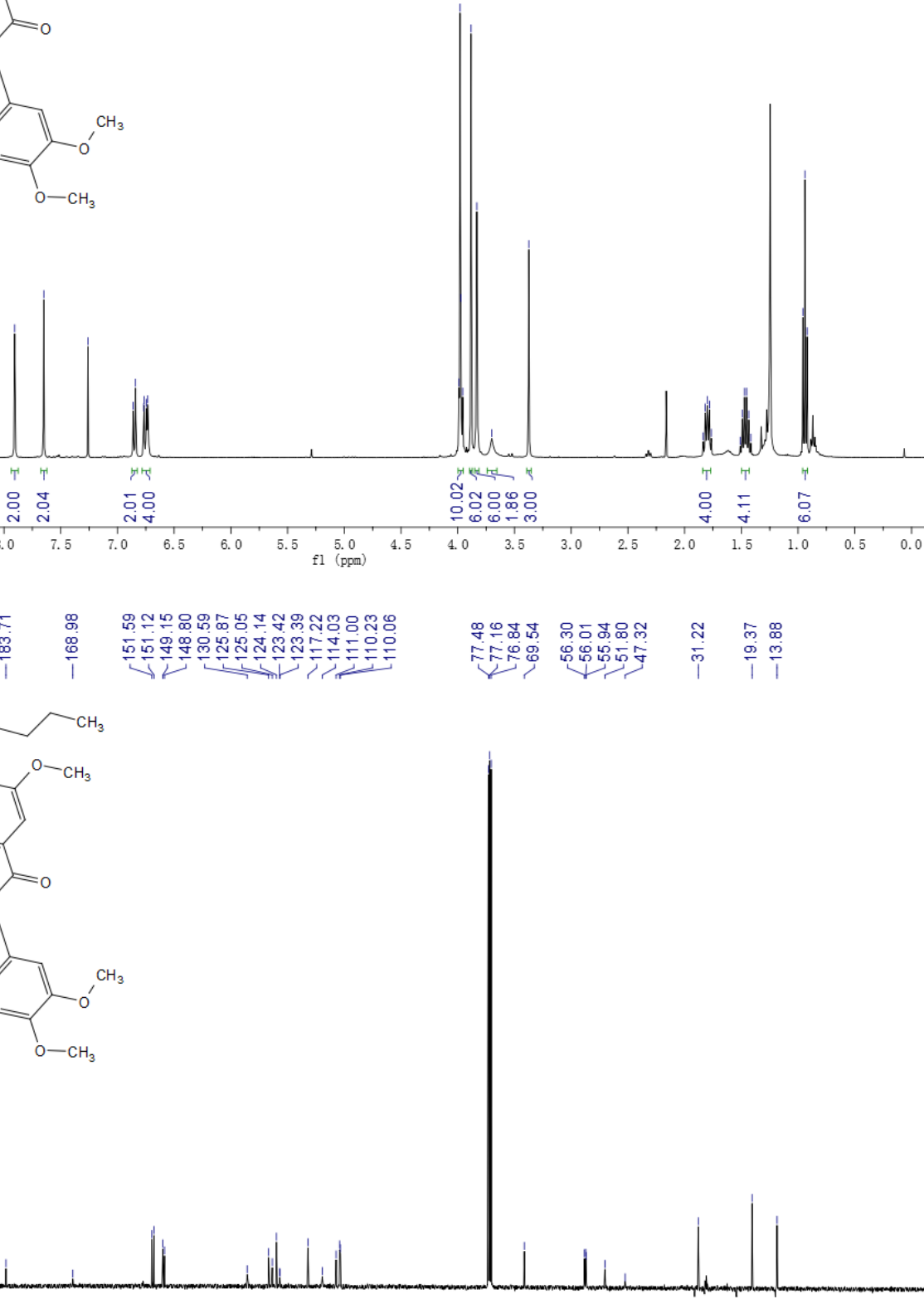

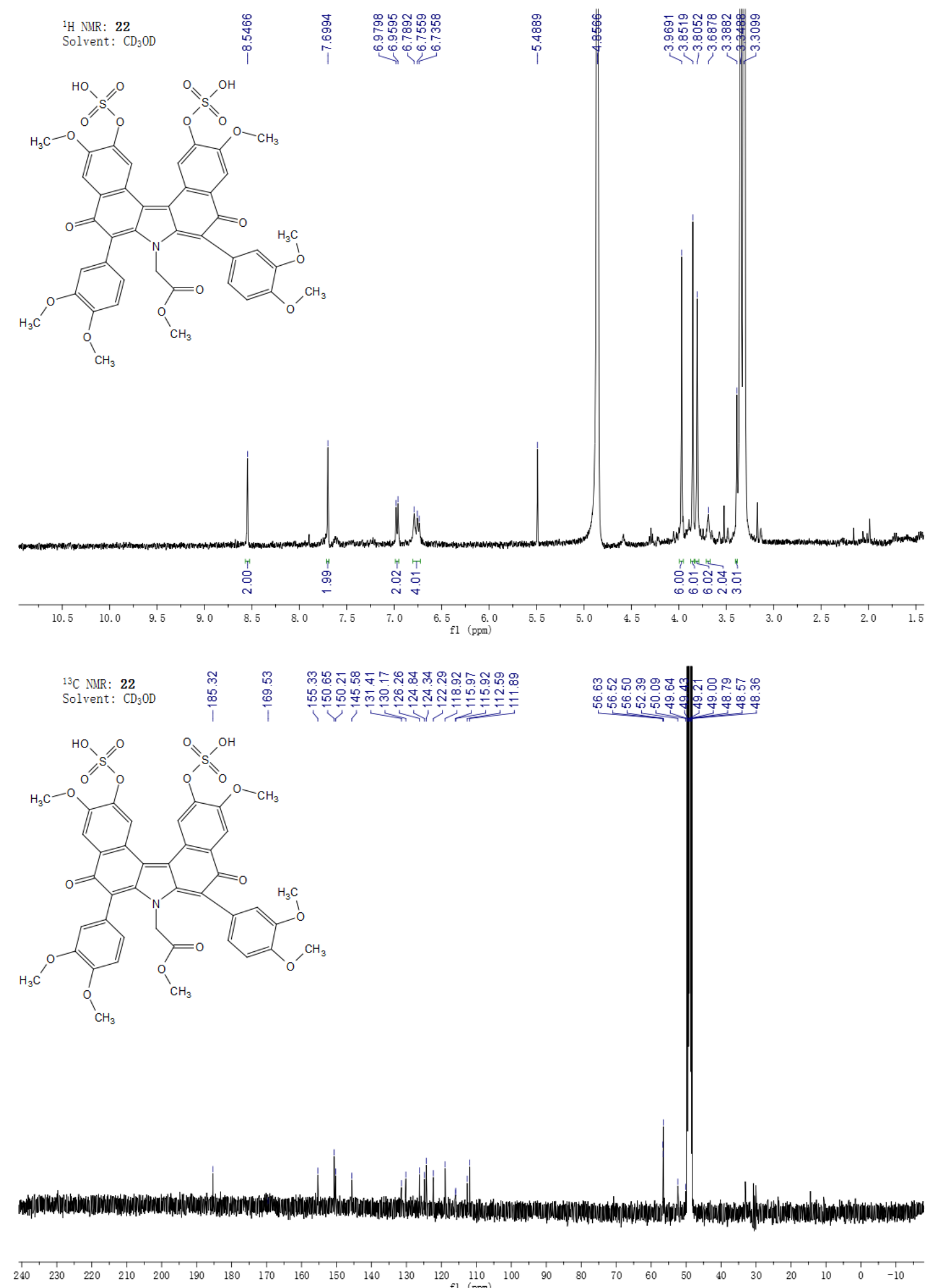


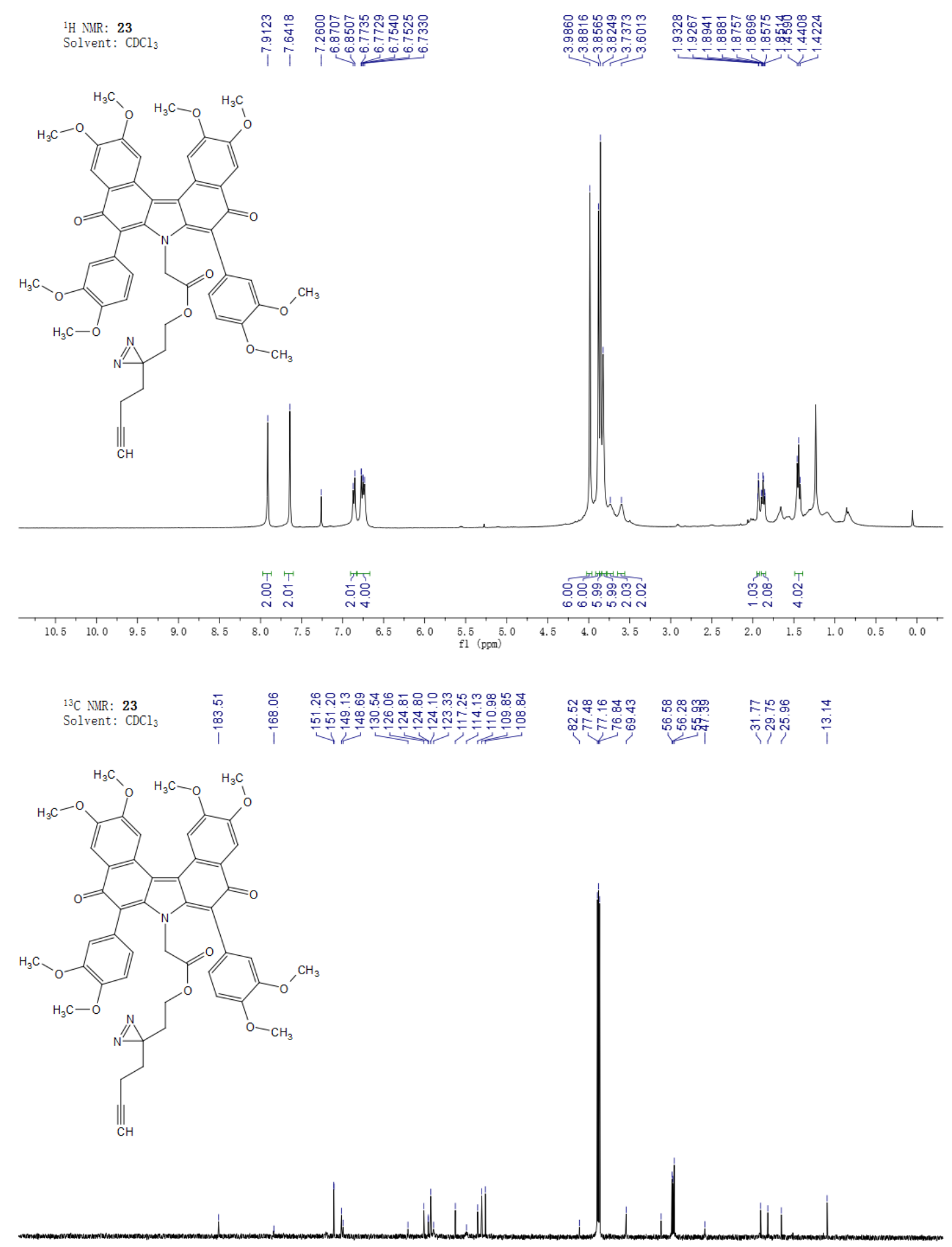

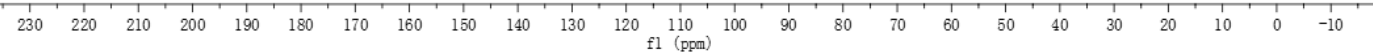



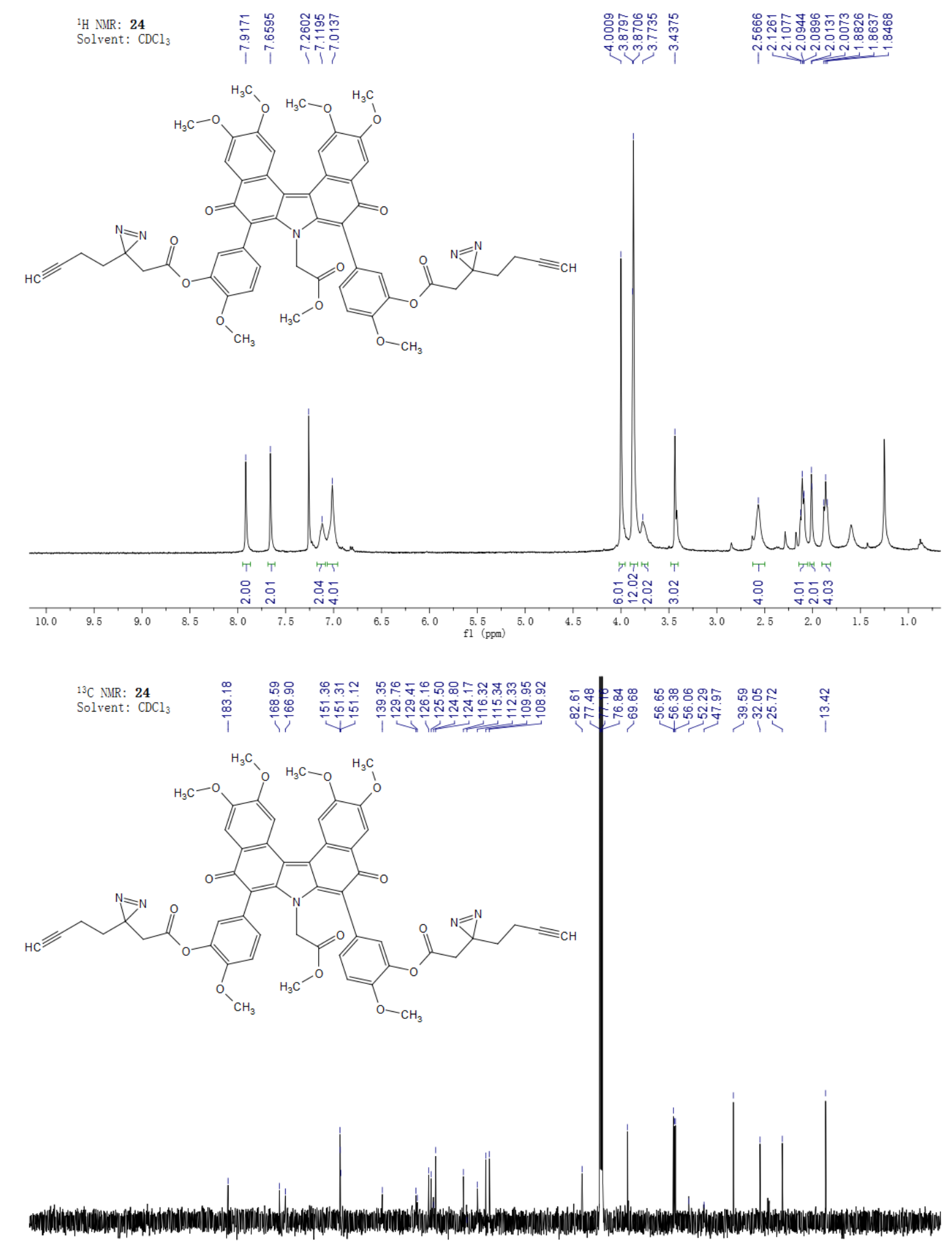

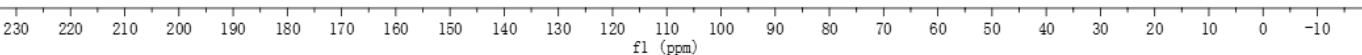




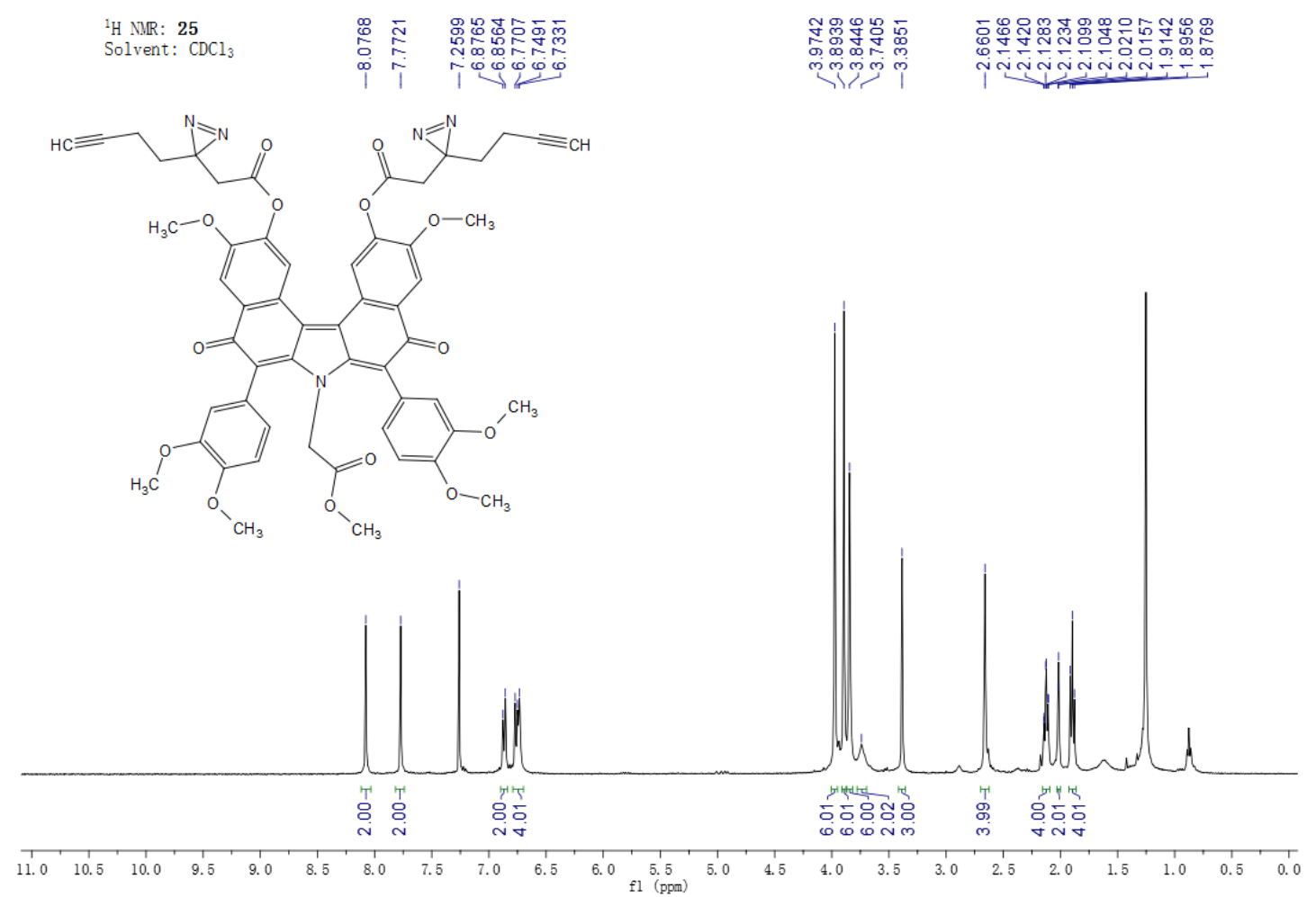

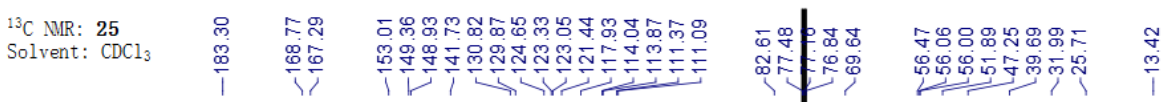
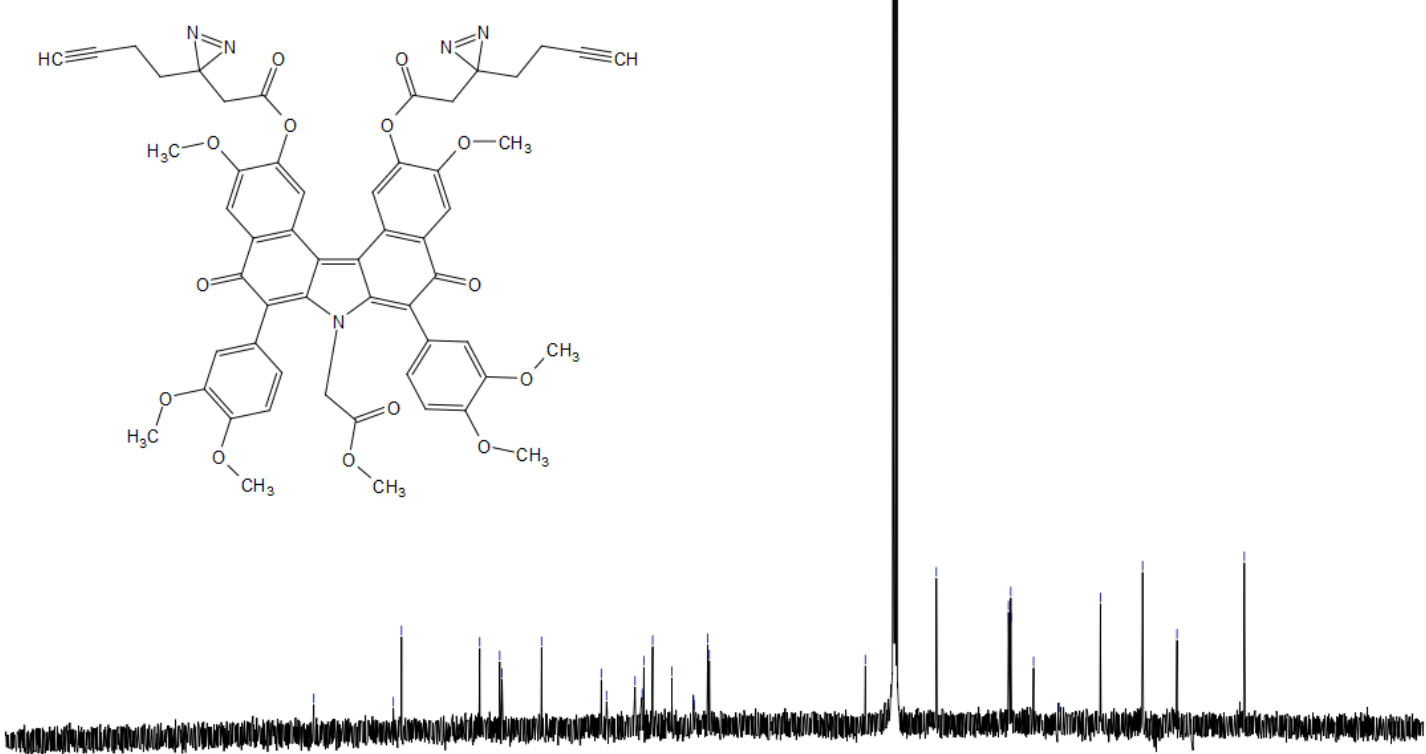

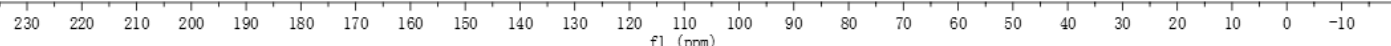

